\title{
A STUDY OF THE ADJUSTMENT EXPERIENCES OF INDIAN INTERNATIONAL STUDENTS \\ IN NEW ZEALAND UNIVERSITIES
}

\author{
BY
}

\section{JOHNSON KUKATLAPALLI}

\begin{abstract}
A thesis
submitted to the Victoria University of Wellington in fulfilment of the requirements for the degree of Doctor of Philosophy
\end{abstract}

School of Languages and Cultures

Victoria University of Wellington

2016 



\begin{abstract}
A review of literature on the experiences of international students reveals their mostly adverse experiences in academic and social lives during their stay in a host country. At the same time, despite facing challenging experiences, international student numbers are rapidly increasing worldwide every year. Moreover, studies in general find that the facilities and services provided by the concerned educational providers are not being utilized by the international students as intended. Within this context, it may be mentioned that although Indian students have been coming to New Zealand for studies since 1951 as a result of the popular Colombo plan, there is no exclusive study on them, although we have such studies on the Chinese and Vietnamese international students.
\end{abstract}

The absence of such a study prevents us from recognising the specific requirements or identifying the unique features of their experiences of adjustment to the new academic and social environment. A few studies on Indian students in other countries have identified some of their difficulties while adjusting to the conditions of host countries. Furthermore, the current statistics of international students in higher education sector worldwide reveal that India is one of the top source countries for international student migration at present and for the future. In New Zealand context, even though India is being considered as an important source country for international students in the university sector, the statistics indicate that the majority of Indian international students are not enrolled in universities. All these reasons indicate the need for a specific study on Indian international students and thus, this study was initiated to explore the adjustment experiences of Indian international students studying in eight universities in New Zealand, focussing on their language, academic and social life experiences.

A mixed methods research approach was employed in this study. A survey questionnaire was used to collect data in the initial quantitative phase, and in the later phase, interviews were conducted for qualitative data collection. Both male and female Indian international students participated in this study. They were studying undergraduate or postgraduate courses at different universities in New Zealand, and belonged to different age groups and religions. Most of them have been studying outside India for the first time in their lives. 
The findings of this study revealed that Indian students have adequate levels of language proficiency; they adjusted well to their academic life; enjoyed their learning experiences in New Zealand; and minimised their psychological issues through utilising social media facilities. These findings differ from those of the other generic research on Asian students. But apart from these positive aspects, the findings also point to a few concerns about their initial adjustment to a new academic system and new social environment in New Zealand, arising from a lack of proper understanding and information. This study therefore suggests a model for better cross-cultural understanding of both the prospective students and the universities as a possible means to minimize the initial difficulties of these students. 


\section{Acknowledgements}

There are many people who supported me throughout this research project and I would like to thank them all. Without their support this thesis would not have ever been completed.

First of all, my profound gratitude goes to my supervisors Prof. Sekhar Bandyopadhyay and Dr. Stephanie Doyle for their wonderful guidance and continuous support in this great journey. Apart from their profound knowledge and experience in this area of research, the extra-ordinary levels of their patience and support helped me to bring this thesis to this point. Their insightful supervision is truly inspirational.

I sincerely thank Associate Prof. Stephen Epstein, Asian Studies Programme for his support and encouragement. My special thanks go to the Head of the School, Academic and Administrative staff of the School of Languages and Cultures, for their wonderful co-operation in carrying out this study. I also thank the authorities and administrative staff of the Faculty of Humanities and Social Sciences, Victoria University of Wellington, for their help and continuous support in the administrative matters. I would like to acknowledge the tremendous support of the Faculty of Graduate Research through several workshops and seminars. I would like to thank the staff of the Library and Student Learning Support Services for their help whenever I required it. My special thanks to the Standing Committee of the Human Ethics Committee of Victoria University of Wellington for granting necessary approvals in connection to my data collection for this study. I am very much grateful to the authorities of Victoria University of Wellington for providing me an opportunity to undertake this study in this prestigious university. At the same time, I would like to acknowledge the co-operation and support from the International Division, Ministry of Education, New Zealand for according permission to use 2007 National Survey questionnaire of international students in this study.

I would like to thank all the participants of this study who contributed their time and shared their experiences. My special thanks go to those of the participants who voluntarily helped me by contacting and encouraging their friends and colleagues to participate in this study. Along with the participants, I thank the academic and administrative staff of various Departments, Schools, Faculties, International Students Offices, and student organisations of all the eight 
universities in New Zealand for their cooperation and help in contacting the targeted group of students for this study. I am also indebted to the support from the publishers of student magazine 'Salient' and community newspaper 'Indian Newslink' in reaching the eligible students for this study.

I specially thank my wife Mrs. Anuja S. S. Vemula, and sons Rev. Jaswanth Aleph Kukatlapalli, and Mr. Jabin Revanth Kukatlapalli for their love, patience, and encouragement to accomplish this project successfully. I believe that if my father Sri Moses Kukatlapalli and father-in-law Sri Christiananda Silvaraj Vemula were still alive today, they would have greatly enjoyed this achievement. I also thank my mother Smt. Vazram, mother-in-law Smt. Francina, and all the members of my extended family for their interest, co-operation, and continuous support.

I sincerely thank my friends in New Zealand and India, Bishop Mark Whitfield, Dr. Petrus Simons, Mr. Hartmut Scharnke, Mr. James Moir, Mr. Andrew John Knackstedt, and Dr. Solomon Raju Basimalla for their encouragement during my study. I also thank Pastor Jim Pietsch and the congregation of St. Paul's Lutheran Church, Wellington, for their good wishes and prayers.

Finally, although it was a challenging experience and task for me, I feel blessed as I was encircled by many encouraging people, and resources. I believe that the outcomes of this study may benefit at least a few international students in future. For this reason, I am grateful to the Father God for providing me this great opportunity, Lord Jesus Christ for His awesome guidance, and the Holy Spirit for the provision of amazing leadership, which all simply made this project possible. All the glory to the Triune God. 


\section{Table of Contents}

Abstract iii

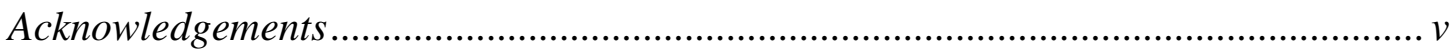

Table of Contents ................................................................................................ vii

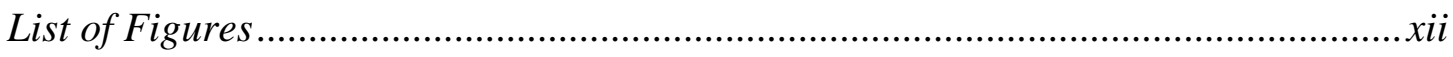

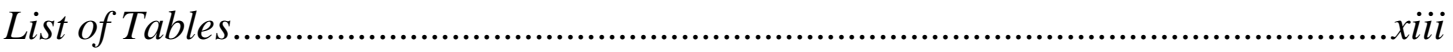

List of Definitions/ Abbreviations .............................................................................

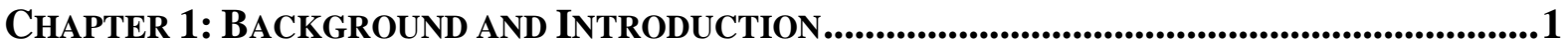

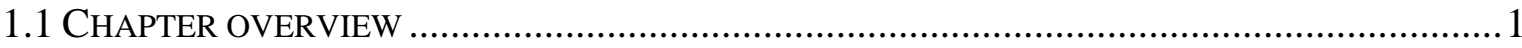

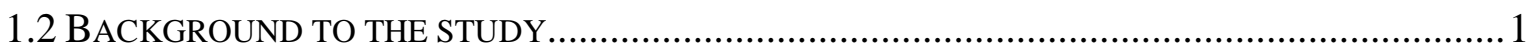

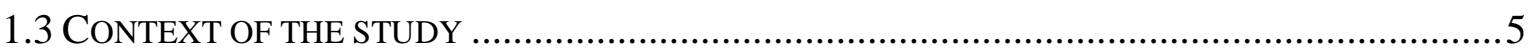

1.4 THE OBJECTIVES AND RESEARCH DESIGN OF THIS STUDY ............................................

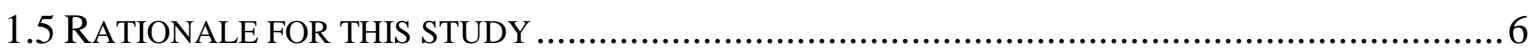

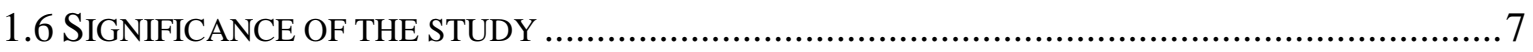

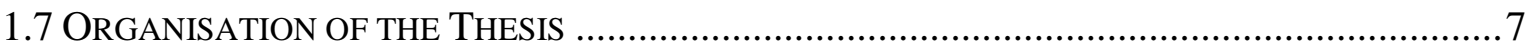

CHAPTER 2: LITERATURE REVIEW ...................................................................................................9

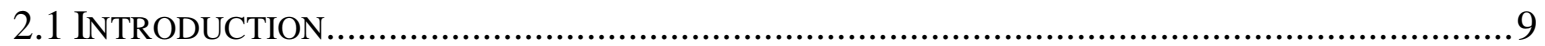

2.2 INTERNATIONAL STUDENT MOBILITY: MOTIVATIONS AND INTERESTS............................. 11

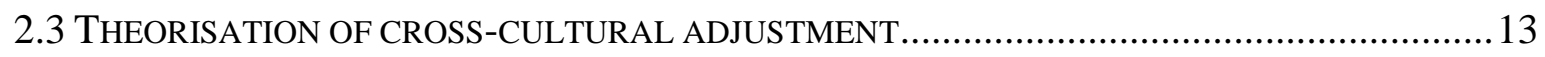

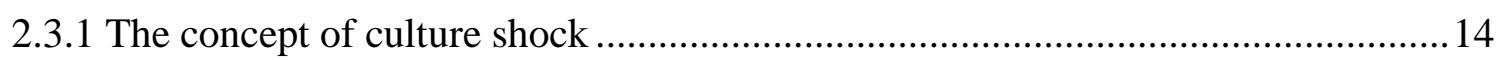

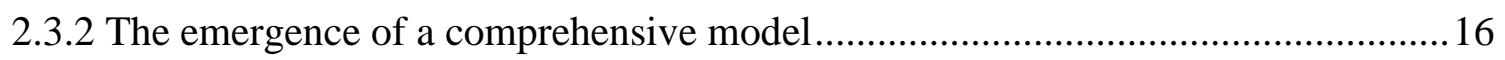

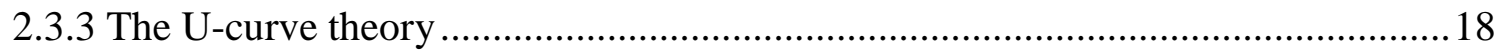

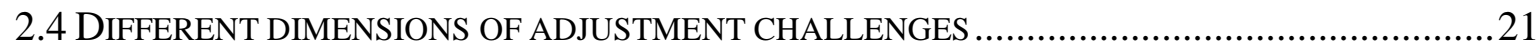

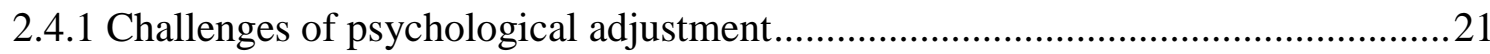

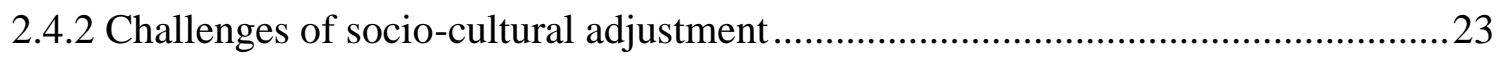

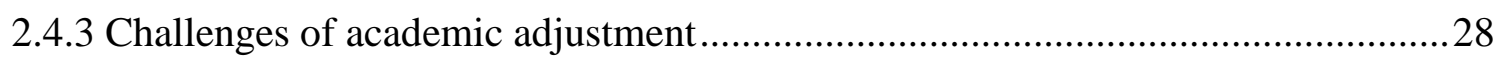

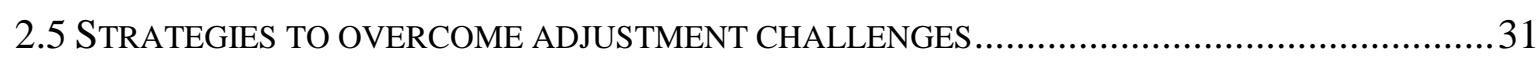

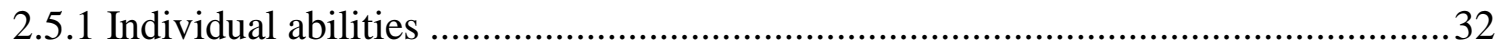

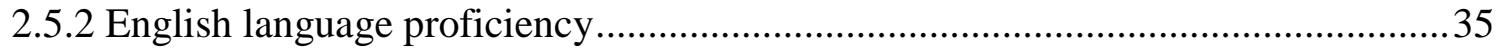

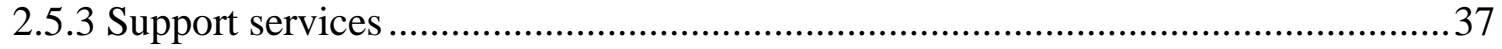

2.6 EXPERIENCES OF INTERNATIONAL STUDENTS IN NEW ZEALAND ..................................42

vii 
2.7 ASIAN INTERNATIONAL STUDENTS IN NEW ZEALAND .... .43

2.8 NEED FOR A STUDY ON INDIAN INTERNATIONAL STUDENTS IN NEW ZEALAND 46

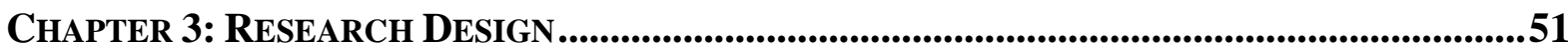

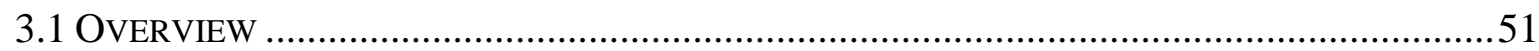

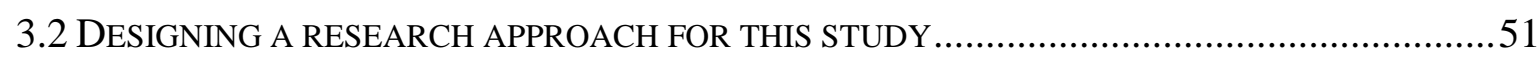

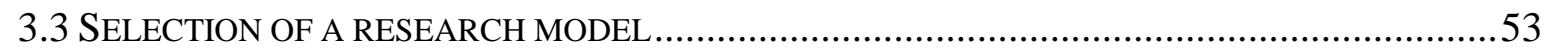

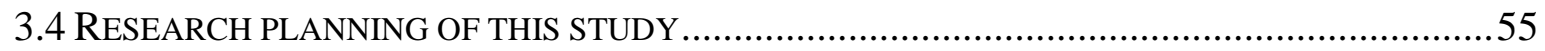

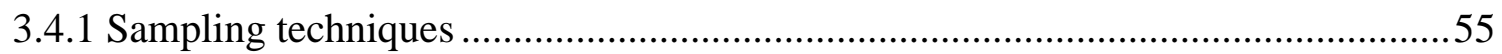

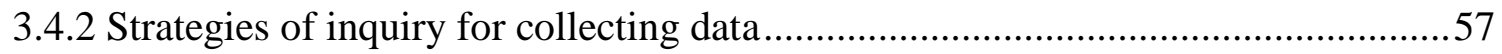

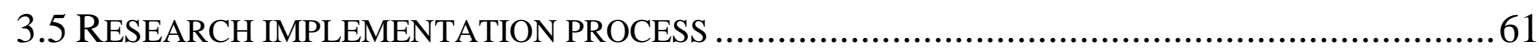

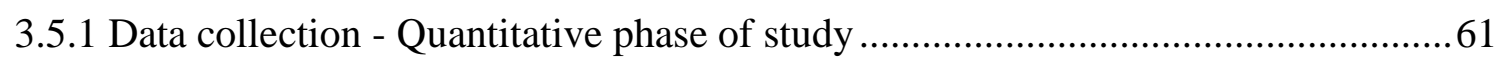

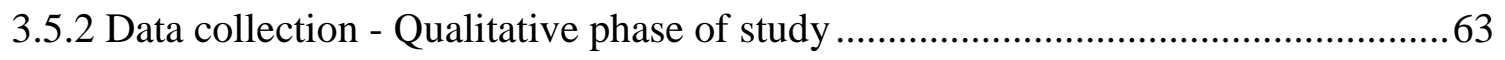

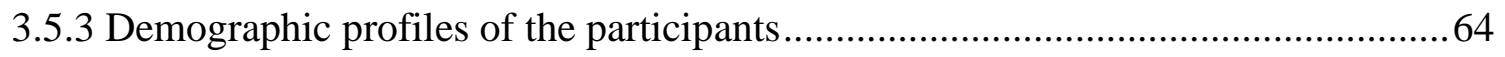

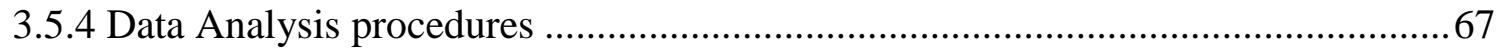

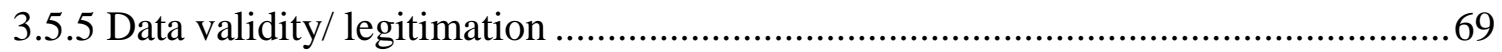

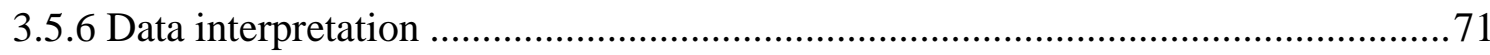

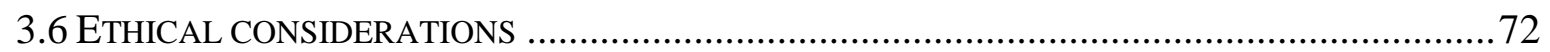

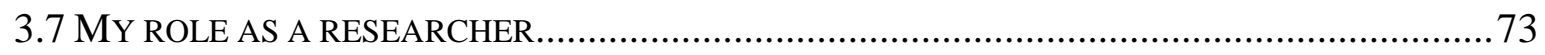

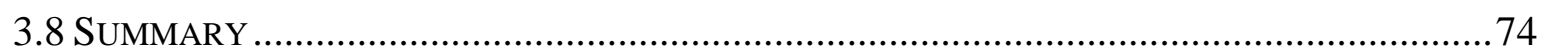

ChAPTER 4: RESEARCh FINDINGS: CHOICE OF NEW ZEALAND FOR STUdies AND ENGLiSH

LANGUAGE PROFICIENCY ....................................................................................................................... 75

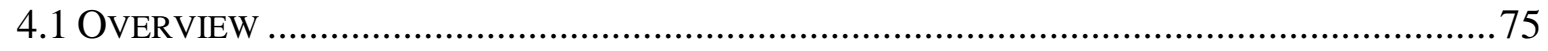

4.2 MotIVATING FACTORS FOR CHOOSING NEW ZEALAND ................................................. 76

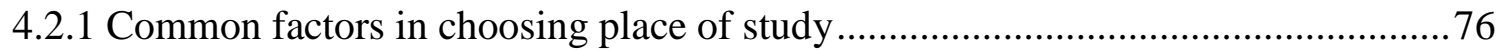

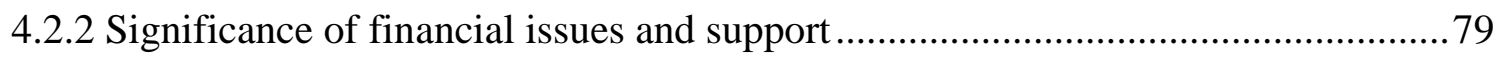

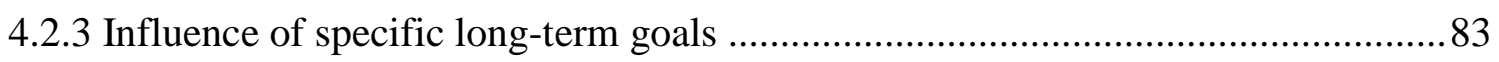

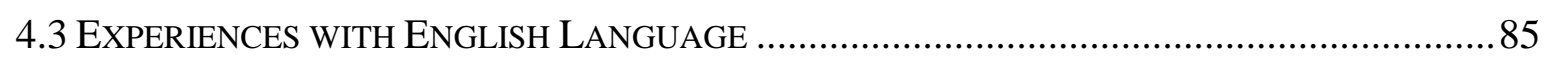

4.3.1 English language proficiency: Before arrival to New Zealand ...............................85

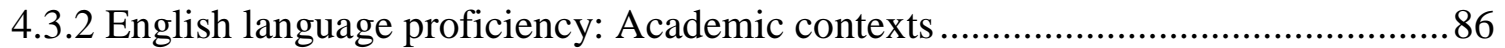

4.3.3 English language proficiency: Non-academic contexts ....................................... 89

4.3.4 Strategies applied to overcome language issues ...............................................91

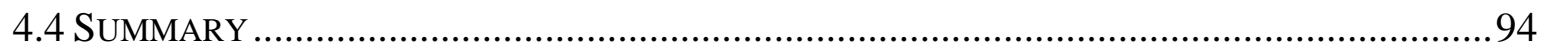

viii 


\section{CHAPTER 5: RESEARCH FINDINGS: ACADEMIC EXPERIENCES AND THE USE OF SUPPORT}

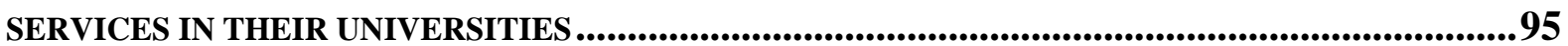

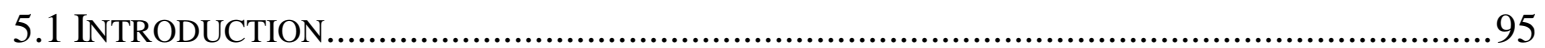

5.2 ACADEMIC EXPERIENCES OF INDIAN INTERNATIONAL STUDENTS ....................................95

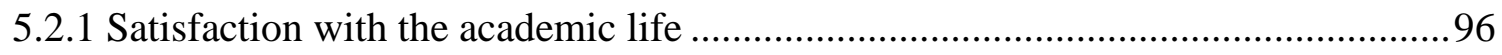

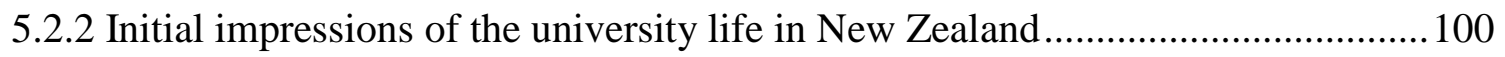

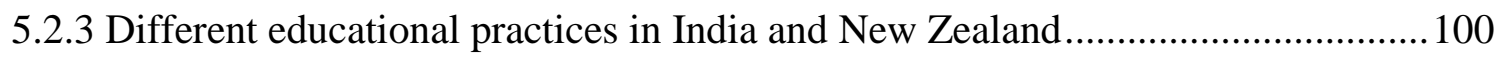

5.2.4 Experiences related to educational activities in New Zealand Universities ..........113

5.2.5 Strategies applied to cope with the academic issues........................................... 120

5.2.6 Suggestions for betterment of conditions related to academic issues.................... 122

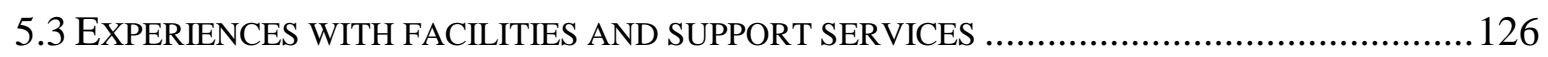

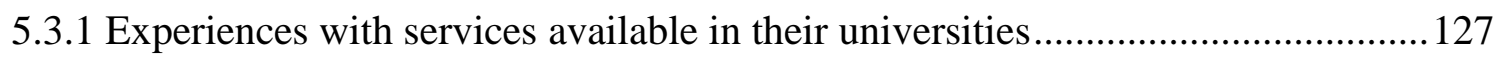

5.3.2 Experiences with the services available from other sources................................ 136

5.3.3 Challenges encountered by the Indian international students ............................... 140

5.3.4 Suggestions for betterment of conditions of the support services ........................ 144

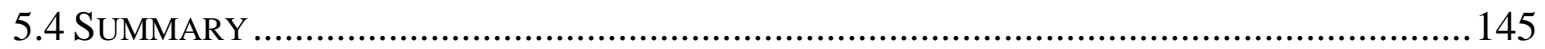

CHAPTER 6: RESEARCH FINDINGS: SOCIAL LIFE EXPERIENCES................................................147

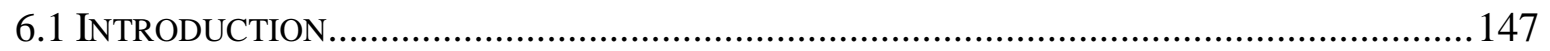

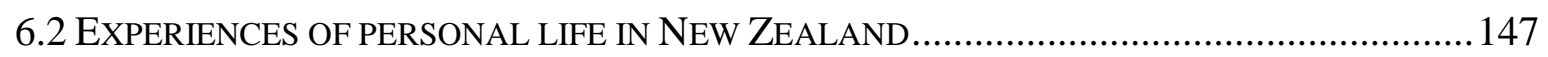

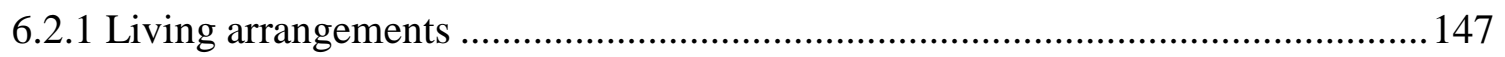

6.2.2 Experiences and challenges with part-time work opportunities .......................... 148

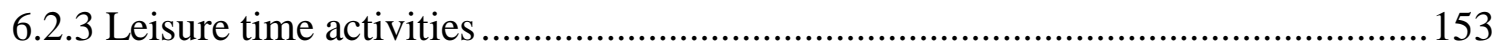

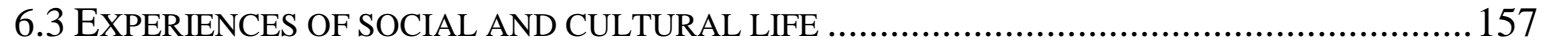

6.3.1 Opportunities for maintaining Indian culture ...................................................... 157

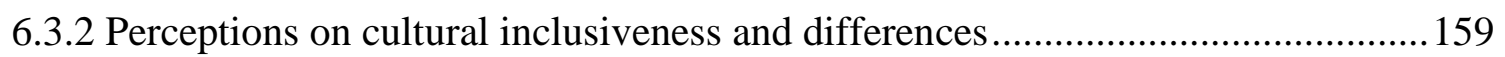

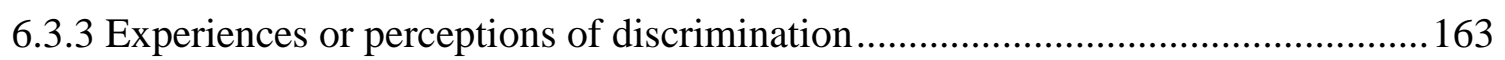

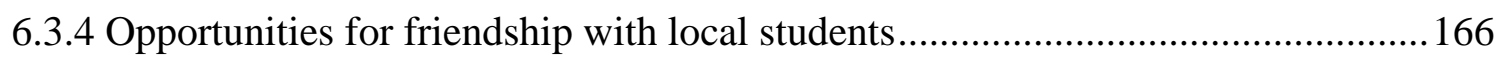

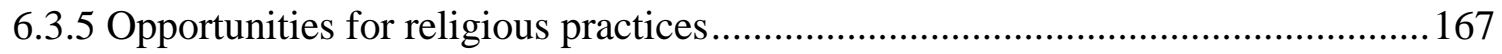

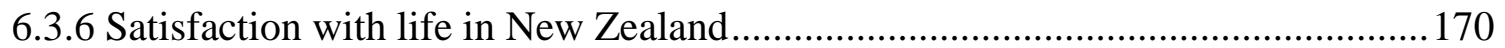

6.3.7 Personal impressions and suggestions for improvement of conditions ................. 173

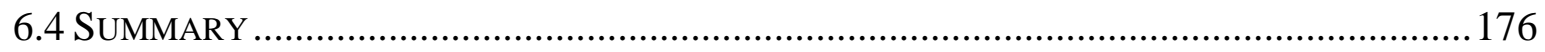


7.1. INTRODUCTION

7.2 FACTORS INFLUENCING CHOICE OF A STUDY DESTINATION

7.3 THE ROLE OF SUPPORT SERVICES IN THE ADJUSTMENT OF INDIAN STUDENTS

7.4 THE ROLE OF ENGLISH LANGUAGE PROFICIENCY

7.5 THE ROLE OF FRIENDSHIPS 191

7.6 THE ROLE OF INFORMATION

7.7 A MODEL ADVISED FOR BETTER ADJUSTMENT OF INTERNATIONAL STUDENTS

7.8 SUMMARY

ChAPTER 8: CONCLUSION

8.1 INTRODUCTION. 207

8.2 PURPOSE OF THE STUDY 207

8.3 SUMMARY OF FINDINGS 207

8.4 THE SIGNIFICANCE OF THE FINDINGS .... 211

8.5 STRENGTHS AND LIMITATIONS OF THIS STUDY 212

8.6 IMPLICATIONS FOR THE UNIVERSITIES AND PROSPECTIVE STUDENTS. 214 References. 217

Appendices 231

Appendix 1 - Survey Questionnaire 231

Appendix 2 - Interview Questions 245

Appendix 3 - Rationale for choosing New Zealand as their study destination 248 Appendix 4 - Aspects of academic life enjoyed most by Indian students in New Zealand

Appendix 5 - Aspects of academic life that appeared to be challenging to Indian students in New Zealand. 252

Appendix 6 - Satisfaction of doctoral/ thesis students on different aspects. 254

Appendix 7 - Factors helping participants to adjust to their academic work 255 Appendix 8 - Levels of difficulty faced by participants in different academic activities

Appendix 9 - Suggestions for universities and international students on academic issues. 257 Appendix 10 - Quality of the services or facilities available in the universities. .258 
Appendix 11 - Frequency of participants' leisure time activities in order of preference .259

Appendix 12 - Participants' perceptions about different sources of support available

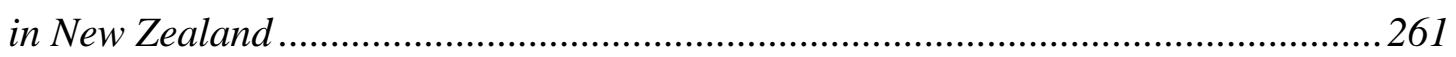

Appendix 13 - Participant Information Sheet for the Survey Questionnaire..............263

Appendix 14 - Participant Information Sheet for Interviews.................................... 264

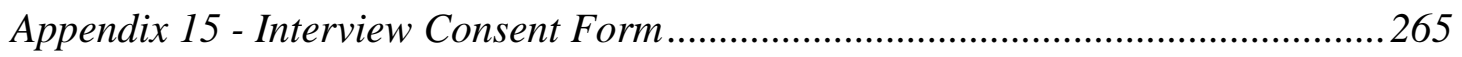

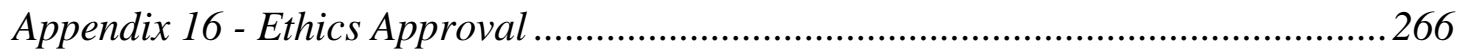




\section{List of Figures}

Figure 4.1 - Difficulty in paying for their education in New Zealand .................................. 80

Figure 4.2 - Self-reported frequencies of English usage prior to study abroad ..................... 86

Figure 4.3 - Self-assessment of English language proficiency in academic contexts .............87

Figure 4.4 - Perceived percentage of communication abilities in non-academic contexts .....89

Figure 4.5 - Perceived percentage of language difficulties in making local friendships ........90

Figure 5.1 - Self-reported satisfaction with the academic experiences ................................96

Figure 5.2 - Levels of satisfaction with different aspects of doctoral/thesis work .................98

Figure 5.3 - Differences in educational practices between India and New Zealand.............. 101

Figure 5.4 - Participants' levels of difficulty with educational activities in New Zealand ... 114

Figure 5.5 - Overall quality of facilities and services in universities .................................. 126

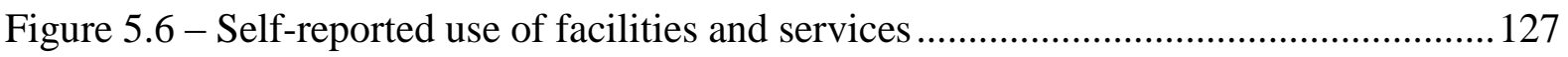

Figure 5.7 - Perceived quality of facilities and services ................................................ 128

Figure 6.1 - Percentage of students eligible to work part-time ........................................... 149

Figure 6.2 - Percentage of details related to part time employment .................................... 149

Figure 6.3 - Percentages showing accessibility to different items of Indian culture .............. 158

Figure 6.4 - Unfair treatment received from the following categories of people .................. 164

Figure 6.5 - Participants' desire to make friendships with New Zealanders .......................... 166

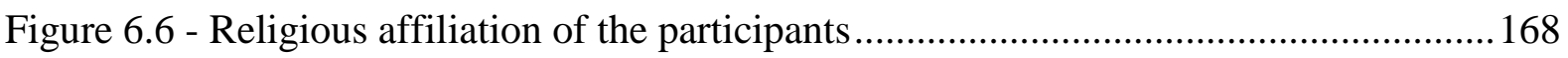

Figure 6.7 - Percentages showing satisfaction levels of life in New Zealand ...................... 170

Figure 6.8 - Percentages of responses showing whether or not encouraging friends and family

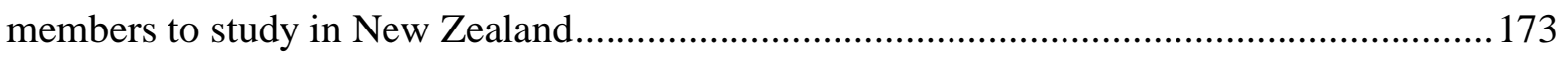

Figure 7.1 - A model for faster adjustment and academic success of international students 201

Figure 7.2 - Activities to be performed by the international students ..................................202

Figure 7.3 - Activities of institutions in providing social support to international students.. 203 


\section{List of Tables}

Table 3.1- Details of the interviewees .66

Table 4.1 - Key factors influencing Indian students to choose New Zealand for study .76

Table 5.1 - Most enjoyed and challenging academic experiences reported by the participants 102

Table 6.1 - Responses showing change of leisure time activities in New Zealand 154

Table 6.2 - Cultural inclusiveness of the participants in their institutions. 159

Table 7.1 - Activities proposed for prospective international students and educational institutions of a host country 198 


\section{List of Definitions/ Abbreviations}

Asian People from the countries of Asian region are referred to as Asian

GMAT Graduate Management Admission Test

IELTS International English Language Testing System

Kiwi Kiwi is a nickname used for the people of New Zealand

SAT Standard Admission Test - a test to get admission into USA colleges

SJS Student Job Search

TOEFL Test of English as a Foreign Language

UK The United Kingdom

USA The United States of America 


\section{Chapter 1 \\ Background and Introduction}

\subsection{Chapter overview}

This study set out to explore the adjustment experiences of Indian international students studying in New Zealand universities. The current chapter provides the background to the study, emphasising its importance in the New Zealand context. The chapter includes description of the context for this study, highlighting the issues, objectives and study design, rationale and significance of the study. This chapter concludes with an overview of the organisation of the thesis.

\subsection{Background to the study}

International students are important to higher education institutions and to host and source countries (Dolby \& Rahman, 2008; OECD, 2013). In 2014, over four million students left their home countries to study overseas, and it is predicted that this figure will double by 2024 (British Council, 2014; OECD, 2014). As the numbers of international students grow, so too does their impact on the social and economic well-being of home and host countries and higher education systems and institutions.

The current trend is for the mobility of international students to exceed that of general migration, with China and India being the leading source countries for international students (King, Findlay, \& Ahrens, 2010; Verbik \& Lasanowski, 2007). According to data provided by Project Atlas in 2010, 200,621 Indian students were studying abroad, up from 139,459 in 2006 (Project Atlas, 2015). The extraordinary growth of student numbers has encouraged research in higher education focussing on the experiences of international students (Dolby \& Rahman, 2008). Remarkably, while there is an extensive literature on Asian international students (Berno \& Ward, 2004; Butcher \& McGrath, 2004; Campbell \& Li, 2008) and on Chinese 
international students in particular (Campbell \& Jieping, 2006; Ho, Li, Cooper, \& Holmes, 2007; Holmes, 2005), there has been little research on the experiences of Indian international students.

The 'Colombo Plan' has significance for the current study, as it was an initiative that included Indian students going overseas to study, and New Zealand as a host country for such students. The intent of the 'Colombo plan' in 1950 was to improve the economic and social development of member countries in the Asia-Pacific region. Although it aimed at furthering economic, social and technical relations between member countries, it was popularly intertwined with the history of 'international students' in New Zealand (Tarling, 2004). The reason for this was that New Zealanders perceived it as a plan which brought Asian students to New Zealand, rather than a plan to develop relations between Asian countries and New Zealand. The initial member countries were Australia, Britain, Canada, Ceylon, India, New Zealand, Pakistan, but the membership later expanded to non-Commonwealth countries. Under the Colombo Plan, 50 scholarships were approved annually by the New Zealand government for International students. Gradually in later years this provision of aid paved the way for other non-aided international students to come to this country. Eventually, the recent global recognition of the standards of its educational system has made New Zealand a desirable destination for many international students.

In recent years, tertiary educational institutions in New Zealand have experienced a remarkable growth in the number of foreign fee-paying (FFP) students. It was reported that there was a 191\% increase in the number of FFP students in public tertiary institutions between 1994 and 2000 (Ministry of Education, 2001b). Further, 80\% of these students were reported to have arrived from different source countries of the Asian region, particularly from the countries of East Asia and Southeast Asia such as China, Japan, Korea, and Malaysia. Having recognised the benefits of the export education industry, including exposure to other cultures, development of relationships with other countries, and monetary benefits of FFP students, a project was carried out by the International Division of the Ministry of Education to promote export education industry of New Zealand (Ministry of Education, 2001a). Following this a number of policy initiatives were introduced including a code of practice for providers, and in 2006 the introduction of domestic fees for international doctoral students. 
Considering the recommendations from the project, a code of practice for pastoral care of international students in New Zealand was enacted by the government for the benefit of international students in New Zealand (Ministry of Education, 2003). This code provides a set of standards for the educational providers to follow while caring for international students in New Zealand. International students as defined by the New Zealand government are 'foreign nationals who travel to New Zealand for the purpose of education, and/or currently studying on a student permit or domestic passport', excluding permanent residents and citizens of Australia (Verbik \& Lasanowski, 2007, p.36). This is the definition adopted in the current study for international students.

Much of the research on the experiences of international students highlights unpleasant experiences of international students in New Zealand and does not reflect the spirit of the enactment (Campbell \& Li, 2008; Ho et al., 2007; Sawir, Marginson, Nyland, Ramia, \& Rawlings-Sanaei, 2009a). Some studies have focused on concerns of international students in relation to their academic and social life contexts, with a number of them recommending ethnic specific studies to recognise the diverse cultural needs and preferences of different groups, and to facilitate the development of, and access to appropriate services (Berno \& Ward, 2004; Deloitte, 2008).

Within this context, this study aims to examine the adjustment experiences of Indian international students in New Zealand, in academic and non-academic contexts. This study is important as there are several contrary conditions that exist between India and New Zealand. For instance, India is a hugely populated country with more than 1.3 billion people (United Nations-Department of Economic and Social Afairs-Population Division, 2015), while New Zealand is a less populated country with around 4.5 million people (Statistics New Zealand, 2015). Further, New Zealand is an English speaking country, while Hindi is the most widely spoken language in India, although English is also used by some people for communicative purposes. Likewise, there are variances that exist in the climatic conditions, cultures, educational systems, and religious aspects between the two countries. 
Past studies, including the New Zealand Ministry of Education's commissioned national surveys have categorized international students according to their national origin, considering each national community as a homogenous ethnic group (Business and Economic Research Limited, 2013; Deloitte, 2008; Ward \& Masgoret, 2004). But there may be a danger in considering the Indians as a single ethnic category based on their national identity. India represents a plural society, being divided by language, region, religion, caste and class, responding differently to the idea of 'nationalism'. There have been two forms of essentialism concerning their national identity. The colonial stereotype was to think of India as a permanently divided society incapable of imagining national unity. The nationalist imagination on the other hand uncritically assumed the existence of a unified national identity and undervalued the power of social divisions. However, as identities are constructed within particular historical contexts, it is necessary to look beyond these two stereotypes (Bandyopadhyay, 2009). It has been shown that in a diasporic context, in New Zealand for example, the Indians often isolate themselves from the local cultural and religious values, and strive hard to retain their Indian community identity, and have felt proud of their Indian heritage. In this way they have lived as 'Indians' in New Zealand, despite divisions within (Johnson \& Moloughney, 2006). So the Indian international students may still be considered as a single national group; but this study has also tried to interrogate that idea.

However, despite such differences and diverse features, the number of international students coming from India and studying in higher education institutions in New Zealand has grown from 2,599 in 2006 to 11,597 in 2010 (Bandyopadhyay, 2013; Ministry of Education, 2011b). With the enrolment numbers of 11,349 in 2012, India has grown to be the second top source country for New Zealand (Ministry of Education, 2013). The latest statistics of Education New Zealand reveal that India is still the second largest source country for international students to New Zealand, with 67\% growth from the year 2013; the number of Indian international students in New Zealand reached 20,289 in 2014 (Education New Zealand, 2015). Yet, there has been no study to date that have focussed exclusively on Indian international students in New Zealand universities. The current study is confined to issues related to Indian international students studying in universities, and to their academic and social life experiences. 


\subsection{Context of the study}

Since modernity and globalisation have persuaded people worldwide to live in New Zealand, the homogeneous ethnic national identity no longer exists in the country (Liu, McCreanor, McIntosh, \& Teaiwa, 2005). Previously, the Treaty of Waitangi in 1840 between the Maori leaders and the representatives of Queen Victoria had made New Zealand a British colony and a bi-cultural society. Later, the arrival of migrants from the Pacific and Asian regions shaped New Zealand into a multi-ethnic and multicultural society. At present it is a country with different ethnic groups with different cultural traditions, such as Maori, European, Pacific, and Asian (Statistics New Zealand, 2015). There is now an official recognition of this multicultural composition of New Zealand society, with official recognition of ethnic minorities and more tolerance of and deference towards their distinctive ethnic cultures. The trend since the 1990s of supporting ethnic minorities and officially celebrating multicultural events have unmistakably redefined New Zealand as a multicultural society (Johnson \& Moloughney, 2006).

Currently, multicultural environment in higher education institutions is a common phenomenon in many countries ( $\mathrm{Li}, \mathrm{Chen}, \&$ Duanmu, 2010). Among the popular destinations for international students in the world, New Zealand is one with global standards of education. Several factors have been identified as influential in the international students' choice of New Zealand as a place of study, including: English speaking environment, a comparatively peaceful and safe location, and a place of natural beauty (Deloitte, 2008; Malcolm, Ling, \& Sherry, 2004; Ward \& Masgoret, 2004).

Although a number of factors attract international students to study in New Zealand, issues concerning the provision of pastoral care, insufficient attention towards meeting the challenges such as educational needs, social needs and different cross-cultural issues, and low interaction with domestic students have been identified as adjustment barriers (Berno \& Ward, 2004; Butcher \& McGrath, 2004; Campbell \& Li, 2008; Ho et al., 2007; Sawir, Marginson, Nyland, Ramia, \& Rawlings-Sanaei, 2009b). These barriers may cause stress to students and result in serious challenges to their psychological, socio-cultural, and academic adjustment to the new 
environment, and to achieving their primary academic goals. Further, these studies have identified significant gaps between the expectations of international students prior to their arrival in New Zealand and their actual experiences in their post-arrival period. This study hopes to contribute to a better understanding of these gaps and address what is needed to enable Indian international students to successfully adjust to the conditions and to achieve their academic goals.

\subsection{The objectives and research design of this study}

This study has two objectives. The initial objective is to understand the experiences of Indian international students in their academic and social lives while adjusting to the conditions of New Zealand. Secondly, it intends to examine the experiences and perceptions of Indian students on the use and effectiveness of support services in their universities. This study makes an attempt to explore their overall experiences and their level of satisfaction with the provisions of support they have received in New Zealand. To explore the experiences of these students indepth, a two phase mixed methods approach was applied.

\subsection{Rationale for this study}

Since 1951 Indian students have been arriving in New Zealand for educational purposes (Tarling, 2004). Past studies on international students' experiences in New Zealand have mostly focused on either heterogeneous samples or Chinese students exclusively (Berno \& Ward, 2004; Campbell \& Li, 2008; Campbell \& Jieping, 2006; Ho et al., 2007; Holmes, 2005). Generic experiences may not reflect the experiences of all international students, nor of particular groups and hence the need for studies with a particular group of students to highlight their specific needs (Chun \& Poole, 2009; Deloitte, 2008). Although India is now a top source country for international students in New Zealand, no exclusive studies have been undertaken in New Zealand to identify their unique experiences and needs.

By exploring the experiences of Indian international students, this study is designed to contribute new knowledge to the existing literature on the experiences of international students 
in New Zealand. Similarly, the research will contribute to the gap in the international literature on Indian students' experiences of studying abroad.

\subsection{Significance of the study}

Research on international students in and out of New Zealand highlights their adverse experiences. In the absence of studies on any specific group of international students it is very difficult to understand their needs and expectations in an unfamiliar environment. Hence, the outcomes of this study may also help educational providers to understand the concerns of the specific group of Indian international students and to introduce or modify their strategies in providing better support provisions to these students.

In recent years the international student community is expanding and representing a larger segment of the worldwide student community. Nowadays, it is common to notice international students in large numbers at tertiary institutions in New Zealand and elsewhere. The statistics related to the international students mobility in higher education worldwide revealed that there were 2.1 million international students in 2001 (Centre for Academic Mobility Research, 2013), around 2.7 million in 2005 (Verbik \& Lasanowski, 2007), more than three million students in 2010 (Bhandari \& Blumenthal, 2010), and 4.5 million in 2013 (OECD, 2013). Therefore, the continuous increase in international students' numbers in global context draws much attention to additional research to identify their issues and to find solutions to ease their adjustment in a new host country.

\subsection{Organisation of the Thesis}

This study is presented in eight chapters. The current chapter provides the background to, nature and context of this study. Along with these, briefly it conveys information on the objectives, study design, rationale, significance of the study, and the organisational structure of the thesis. 
Chapter two presents the literature review. This chapter draws on the literature to provide a discussion of different motivational factors involved in international student mobility, theorisation of cross-cultural adjustment, different dimensions of adjustment challenges, appropriate strategies to overcome these challenges, and experiences of international and Asian students in New Zealand. Lastly, the discussion concludes by establishing a need for this study, and with the two research questions.

Chapter three deals with the research design for this study. This chapter initially describes the procedural criteria for selecting an approach and model for this study. Later, it explains how this study was planned and conducted, including the details of data collection, analysis, and interpretation procedures. Further, this chapter illustrates the principles observed in establishing the ethical standards and trustworthiness of this study.

Chapters four, five and six report the findings of this study. Chapter four presents the findings related to the motivational factors in selecting New Zealand as a study destination and language experiences in an English speaking environment of New Zealand. Chapter five provides details of the academic experiences of participants, along with their perceptions on the use and effectiveness of support services in their universities. Similarly, chapter six describes the findings on their social life experiences in New Zealand. The concluding parts of this chapter contain different suggestions offered by the participants for the educational institutions and prospective international students for the betterment of conditions in New Zealand.

Chapter seven comprises discussion on the study findings. The findings related to different themes are discussed in relation to the reviewed literature. Besides the discussion, this chapter provides evidential data on the contribution of this study to the existing literature on international student experiences in New Zealand. Further, this chapter highlights unique features of the participants of this study. Finally, this chapter presents a model for faster adjustment and academic success of international students in a new environment. It is based on the observations of the reviewed literature and the findings of this study.

The final chapter eight presents the summary of the findings from this study along with the details of the significance, strengths, and limitations. Later, this chapter provides a few implications for the universities and prospective international students. 


\section{Chapter 2 \\ Literature review}

\subsection{Introduction}

After the Second World War, a number of governments initiated policies attracting international students to their educational institutions. For some countries, such as the United States, Britain, Australia and Canada, education became an important export industry (Ward, Bochner, \& Furnham, 2001). The global trend shows that the growth rate of international students' mobility is even exceeding the general migration rate (King et al., 2010). In the higher education sector the figures show continuous increase of international student numbers as mentioned in the earlier chapter. Further, it is reported that a significant number of these students are of Asian origin, mostly from the countries like China, India and Korea (OECD, 2013).

The top source countries for these students are China and India (King et al., 2010; Verbik \& Lasanowski, 2007). A recent report on the UK higher education sector has forecasted that by 2024 the number of outbound postgraduate students from 23 source countries will rise by 335,000, and of them India and China will contribute $36 \%$ and 33\% respectively (MacGregor, 2014). Even though the current enrolment figures at tertiary level show a greater number of Chinese international students, it is predicted that the number of Indian students will surpass Chinese students in future. This is because by the year 2025, it is predicted that the Indian population in 15-24 age group will increase from the present 232 million to 245 million, while the same age group population in China will decrease to 172 million from the present 212 million (Gopinath, 2015).

The continuous increase of international student numbers worldwide has predictably stimulated a wide variety of research, particularly in relation to the tertiary education sector (Dolby \& Rahman, 2008). One of the motives behind this research is believed to be economic, i.e., how to better deliver higher education as a commercial commodity in a globally expanding competitive market. This research was meant to identify the needs and concerns of international 
students during their study, recommend measures to enrich their learning experience with better provision of services and thus to make the tertiary education providers more competitive in the global education market.

The extensive literature that is now available on the international students at tertiary level, indicates that international students face more problems at academic, social and cultural levels than the domestic students, such as, excessive use of health services, and low use of support services in their educational institutions (Ward, 2001). Andrade (2006b) in her review of empirical studies also identified that international students experienced greater difficulties compared to domestic students in making psychological, social, and academic adjustments. Her review revealed that language proficiency levels and cultural differences determined their levels of difficulties. Based on the information provided in the studies she reviewed, Andrade defined international students as individuals pursuing higher education on student visas, who are also non-native English speakers. However, we should also note the problem involved in this definition, as many international students these days studying in countries other than their countries of birth are indeed native English speakers. And many non-native speakers are also proficient in English. But Andrade's definition can be applied more generally to Asian international students.

Berno and Ward (2004) report which focused on the tertiary level Asian students' psychological, socio-cultural and academic adaptation experiences in New Zealand, has pointed out that the participants' experiences under-met their expectations in all these aspects during their initial three months of stay and study. Further, this report emphasised that Asian students experienced more academic difficulties compared to their peers from western countries. The situation was mainly attributed to their lower levels of English language proficiency. Additionally, Asian students experienced greater levels of discrimination compared to other international students. They also expressed strong desire to develop contacts with New Zealand students compared to other international students from western countries, but had limited success. These experiences, particularly at the initial months of their stay indicate the vulnerability of the Asian students in a host country like New Zealand.

Based on these insights, both these studies mentioned above recognised the dire need for assisting international students with better support, particularly in improving their English 
language ability (Andrade, 2006b; Berno \& Ward, 2004). Further, Berno and Ward (2004) reported that social support facilities provided by the institutions were less used compared to other sources of social support such as family and friends. They categorized 'social support' as either instrumental or emotional. While instrumental support provides students with basic information and guidance to deal with their practical problems, emotional support ensures better psychological and socio-cultural adjustment. In this context, it was reported that Asian students felt that their institutions provided inadequate emotional support compared to instrumental support. The lack of adequate social support unfortunately is likely to increase acculturative stress among international students (Poyrazli, Kavanaugh, Baker, \& Al-Timimi, 2004).

The studies mentioned above show that a majority of international students globally are from Asian countries, and they are generally non-native speakers of English. However, their preferences are for higher level courses (post-secondary school) in an English speaking environment. As a result, as most of the literature on Asian international students' experiences has revealed, they struggle against a variety of adverse conditions, as described above. Furthermore, the literature indicates that international students are being targeted in furtherance of the economic interests of the host countries (Dolby \& Rahman, 2008). Given these challenges and adversities, one might expect that there would be a steady fall in student numbers. But surprisingly, the number of Asian international students has been increasing in recent years, as mentioned above. To understand the reasons for this strange situation, different factors and interests stimulating student mobility are considered and explained below.

\subsection{International student mobility: Motivations and interests}

The general expectations that motivate students to study abroad are many: intercultural exposure, enhancement of language skills, better qualifications and career prospects (King et al., 2010; OECD, 2013). Globally, student mobility has gained prominence in the higher education sector (Chan, 2012). Host countries have vested interests in offering educational opportunities to international students. According to Verbik and Lasanowski (2007), such vested interests include both short-term and long-term benefits international students bring to the host countries. The immediate boost to income is the short-term benefit, while the use of 
their skills for the benefit of the host country is considered to be long-term benefit. While this observation informs us of the immediate interests of the host nations in attracting international students, it also implies that for protecting and furthering those interests it is also necessary to take note of the experiences of current international students, as these are likely to influence future student mobility. In short, the information can help host nations to design new strategies or revise the old ones to stay competitive in the international education market.

From the perspective of international students, a personal journey to a foreign country for studies may not simply be confined to a short term goal of attaining an educational qualification, it may extend to further long term goals (Baas, 2010; Bandyopadhyay, 2010; Bethel \& Ward, 2014). For example, Baas (2010) study in Australia conducted on Indian international students reported that other than the primary educational goal, the participants had other predetermined goals such as employment and immigration. Likewise, Bethel and Ward (2014) study on tertiary international students in New Zealand reported similar intentions of Indian international students. As these studies have pointed out, international students also have short-term and long-term goals when they enter a new country. However, the validity of the above claims needs to be re-examined as all these students may not have similar goals while considering different study destinations. And then the levels of satisfaction of the current students in a host country can shape and influence their subsequent life decisions and consequently can also influence future flow of international students to that particular host country. Therefore, it is necessary to identify their expectations by looking at their own perceptions.

In addition, studies have indicated that student mobility to a new country is influenced by the prospects of new opportunities that such a journey offers, such as exposure to a new culture and language (Doyle et al., 2010; King et al., 2010). This phenomenon is more evident in student exchange programmes, because such programmes are specifically designed to provide opportunities to domestic students to travel overseas for such exposures. An empirical study conducted in New Zealand aimed at identifying factors that promote student exchange programmes revealed that exposure to a different culture and language was the primary reason for the participants to go for student exchange programmes (Doyle et al., 2010). However, while this study reflected the views of domestic students of New Zealand, it will be worthwhile to find out what the international students think of New Zealand providing them exposure to a 
different culture and language. Additionally, international students usually stay for a longer period in a host country compared to exchange students, and so they are expected to have specific strategies to handle any issues that may arise because of unfamiliar culture and language. The exploration of different strategies applied by these students to overcome these challenges may be beneficial for future international students.

Beyond the previously mentioned mutual benefits for both the host country and international students, such mobility as observed in the literature, is also beneficial to the source country. This is even truer for those countries that encourage their students to take up studies overseas. For example, Tremblay (2005) notes that when opportunities in the home country are insufficient to the demand, such countries encourage students to undertake studies overseas. As she argued, such an encouragement would entail benefits to the sending country upon the return of their students. According to her, the list of benefits to the sending countries are: transfer of technology learnt overseas, economic investments, and opportunity to participate in education of global standards at little or no costs to the state (since the individuals who study overseas are often self-financed). Further, by encouraging its students to undertake overseas study, the source country may promote healthier relationships with the countries involved in international education market. In this context, the views and experiences of the participating students may be helpful in building cultural bridges between countries.

Overall, the above observations inform us of many reasons for international student mobility other than the personal interests of the students. However, when a student enters a host country, he/she needs to adjust to the conditions in a different and unfamiliar environment to achieve the primary academic goals. The experiences may be either positive or negative and may provide information alerting us to any concerns or challenges that could influence future inflow of international students.

\subsection{Theorisation of cross-cultural adjustment}

Generally, the research on 'international students' experiences' constitutes a distinctive literature, a component of a research approach labelled 'internationalisation of higher education' (Dolby \& Rahman, 2008). Further, this approach is a sub-set of 'research in 
international education', which is an integral part of a broad umbrella theme 'Educational research'. However, international students are a distinct academic subject of research, similar to other cultural travellers, such as immigrants and business people, on cross-cultural transition and adaptation (Ward et al., 2001). Because of their sojourning status, international students, unlike domestic students, experience more problems such as language issues, cultural differences in both academic and social life, social difficulties. Besides this, as they are burdened with additional academic stress and low levels of personal resources compared to other sojourning groups, international students experience higher levels of acculturative stress (Poyrazli et al., 2004). Being sojourners in a different culture, they need to undergo crosscultural transition at a fast pace within a limited time span, to achieve their pre-set academic goals effectively. To achieve positive outcomes, they require better support services to cope with the conditions. However, as the adjustment issues of international students are aligned to cross-cultural transition, three key theoretical strands from the relevant literature are explained below before looking at their adjustment experiences in detail.

\subsubsection{The concept of culture shock}

When a person encounters a new culture, in the midst of an unfamiliar environment, that person will experience aggravation and anxiety (Oberg, 1960). Oberg labelled this experience as 'culture shock'. Oberg viewed the response of people to 'culture shock' in terms of occupational disease, a condition that a transitioning sojourner goes through in a new place. Oberg (1960) noted that the effect of 'culture shock' in physical and psychological terms might sometimes even lead an individual to reject the host culture while stressing the superiority of one's home culture, and thereby significantly affecting any positive cross-cultural transition of that individual in a new host country.

Contrary to this view, Adler (1975) saw such a cross-cultural transition not only as an event causing frustration, but also helping an individual to widen his/her knowledge while undergoing this process. Therefore, according to Adler, the response of individuals to the 'culture shock' may no longer be understood just in terms of an occupational disease as Oberg viewed it, but it could also be seen as a positive experience broadening the knowledge of an individual. This is because the transition process would transform a person from being monocultural to inter-cultural. Other than this, he believed that when an individual encounters a new 
culture, that person not only learns about a diverse culture, but also develops an awareness and understanding of one's own ethnocentrism. Adler's views were further echoed in a later study indicating that cross-cultural transition experience might stimulate a person to learn from the new experiences (Zapf, 1991). Thus, culture shock is no longer seen as a psychiatric disorder or occupational disease, but is considered as a routine phase encountered by any person walking into a cross-cultural situation (Mumford, 1998).

Church (1982) indeed highlighted that many studies he reviewed did not refer to the concept of 'culture shock'; instead, to understand the experience of international students they used other parameters such as academic achievement, satisfaction related to personal and social aspects of life, and problems. So, to resolve their issues or to achieve satisfaction with their personal, social and academic lives they need to adjust to the conditions of the new environment. Such an adjustment for international students, who spend a shorter period of time in a new cross-cultural environment, was referred to as a 'sojourner adjustment' by Church. This shorter period of stay is typically between six months to five years (Ward et al., 2001). Usually, international students enter a new country with an aim to achieve specific academic goals within limited periods of stay, besides adjusting to the conditions of that host country. This situation obviously leaves them in a relatively vulnerable situation to experience additional pressures and challenges, compared to other cross-cultural travellers, requiring a different process of adjustment.

Searle and Ward (1990) also argued that the concept of 'culture shock' could not sufficiently explain the nature of difficulties or enlighten us about the requirements of sojourners like international students. Subsequently, Ward and Kennedy (1993) proposed the use of terms 'adaptation' or 'adjustment' to refer to such cross-cultural transition. In a similar fashion, Zhou and her colleagues (2008) in their review of literature contended that, 'culture shock' as a notion was no longer viewed in the literature as a passive phenomenon (i.e. passive recipients of trauma). However, it was transformed to include aspects of contact-induced stress together with skill deficit, which can be coped, thus accepting the terms 'adaptation' and 'acculturation'. Currently, the extant literature uses these terms to explain the adjustment process, thus making it the standard in recent cross-cultural literature. Apart from the terminology employed to 
analyse cross-cultural transition, the literature also pointed to certain frameworks in which cross-cultural adjustment is perceived.

\subsubsection{The emergence of a comprehensive model}

Oberg's (1960) observations on the cross-cultural adjustment of an individual included negative impacts of 'culture shock' and subsequent coping strategies employed by an individual to overcome these challenges in a new host country. Oberg believed that individual abilities (such as language proficiency) of a person along with the improved social interactions with host nationals, and familiarity with the surroundings over a period of time would minimise the effect of 'culture shock' and would help a successful transition. Resonating along with Oberg, Church (1982) observed that language plays a role in adjustment process since it could influence social relations. While the observations of Oberg and Church are valued in the discussion of cross-cultural adjustment, the limitation of their respective frameworks lies in viewing the concept of 'adjustment' as a unified process.

Contrary to the above view, later studies have perceived 'adjustment' in a different way. Having surveyed the cross-cultural literature extensively, as well as taking into account their own findings from an empirical study, Searle and Ward (1990) proposed the concept of adjustment as a two dimensional process, the psychological and socio-cultural. The psychological adjustment relates to individual satisfaction or having a feeling of 'well-being', within the theoretical framework of 'stress and coping model'. On the other hand, the sociocultural adjustment relates to the ability of an individual 'to fit' into the new host society with the required skills, pertaining to the 'social learning' theoretical framework. The 'social learning' theories take into account such factors as 'cognition' and 'behaviour' of a person seeking to adjust to a cross-cultural context (Black \& Mendenhall, 1991).

Subsequently, Ward and her colleagues (2001) developed a broad theoretical model to better understand the cross-cultural adjustment process. They further extended the theoretical frameworks of 'stress and coping' and 'social learning' models by including 'social identification' theories. Together these three models were known as 'comprehensive model' (Ward et al., 2001). The three models originate from social psychology, early clinical approaches, and cognitive based research. Although, these models have origins in other fields, 
these approaches have become prominent in the field of education as well (Zhou et al., 2008). The comprehensive model suggests that adjustment should be viewed as a process consistent with psychological and socio-cultural responses to handle cross-cultural issues, rather than merely as passive condition of a sojourner experiencing a new environment.

Although the 'comprehensive model' consisted several strengths and considered as a dynamic theoretical framework to understand the acculturation process, it has also received criticism for lacking clarity, particularly in explaining the connection between psychological adjustment, socio-cultural adaptation and 'academic' adaptation process of international students (Zhou et al., 2008). Further to this, Zhou and her colleagues (2008) felt that this model did not properly accommodate the cognitive elements of adaptation in the acculturation process. Consequently, Zhou and her colleagues emphasized the need for further explanation of the psychological and socio-cultural adaptation processes, particularly with reference to 'pedagogical adaptation'. In fact, Zhou and her colleagues found that 'culture synergy' concept proposed by Cortazzi and Jin (1997) was an appropriate model for understanding pedagogical adaptation process. The 'culture synergy' model highlights the 'cross-cultural understanding' of both teachers and international students, and illustrates how they both may mutually benefit in their teaching and learning roles.

Prior to Zhou and her colleagues' study, Furnham (2004) had looked at the effect of culture shock in the learning process of international students and projected Bochner's functional model of different friendship patterns that could provide effective support to them. This model propagates that monocultural, bicultural and multicultural friendship networks collectively could help international students to overcome their psychological, academic, and social life challenges respectively. Then again, Zhou and her colleagues (2008) countered Furnham's proposal by emphasising two reasons. They contended that the host-national friendship networks alone could help these students to overcome their issues of psychological, sociocultural and academic aspects. But, despite that advantage, the extant literature indicates limited interactions between the international students and host-nationals. Therefore, Zhou and her colleagues strongly recommended 'culture synergy' model as an appropriate model for studying pedagogical adaptation process of international students. 
As Zhou and her colleagues rank the 'culture synergy' model over the 'comprehensive model', this marks a way forward for our analysis of adjustment process of international students. While all previous studies placed the responsibility of adjustment principally on an individual sojourning in a new host country, the 'culture synergy' model as suggested by Zhou and her colleagues extended that responsibility beyond an individual student to even include teachers of the host nation, suggesting a mutual adjustment process in a way. As it appears from the recommendations of a number of empirical studies (discussed in the next section), the dominant analytical trend now is in line with the views of Zhou and her colleagues.

\subsubsection{The U-curve theory}

The literature on cross-cultural adjustment informs the analysis and support towards the popular 'U-curve' theory, which Lysgaard has been credited for establishing (Church, 1982). This theory proposes that a sojourner in a new place often experiences four stages of adjustment. These stages are: the honeymoon stage, which refers to the excitement and fascination of individuals in the new host country. This is followed by a stage of culture-shock referring to the disillusionment and frustration of facing the challenges of a new place. This is in turn followed by a stage of adjustment referring to the coping strategies gradually developed to adapt to the new place. Finally, comes a stage of mastery where a person effectively functions in the new environment. Apart from the 'U-curve' theory, Church (1982) in his review described another prevalent theory of adjustment namely ' $\mathrm{W}$-curve' theory proposed by Gullahorn and Gullahorn (1963). This theory adds another phase of shock experienced by sojourners when they re-enter their home culture after spending some time in a foreign country.

Even though both theories explained the cross-cultural experience of an international sojourner, 'U-curve' theory was widely received as well as rejected in the scholarly circles (Black \& Mendenhall, 1991). Having reviewed the available literature on both sides of the debate, Black and Mendenhall concluded that it was 'premature' either to support or reject 'U-curve' theory. This is because, they argued, 'U-curve' theory when complemented by 'social learning' theory (consisting of both cognitive and behavioural theories) can be a helpful resource for understanding the adjustment process. According to their view, social learning theory suggests a way of understanding 'U-curve' theory because it explains the why and how of the adjustment 
process. So, Black and Mendenhall (1991) argued, it would be helpful to follow 'U-curve' theory in order to comprehend adjustment process.

However, 'U-curve' theory also drew criticism from a few scholars (Brown \& Holloway, 2008; Church, 1982; Ward, Okura, Kennedy, \& Kojima, 1998). For instance, Church (1982, p. 543) deemed it 'weak, inconclusive and over generalised'. He argued that many researchers using this theory failed to substantiate it with empirical evidence. He highlighted that most of the studies related to sojourner adjustment applied concepts drawn from the host culture and applied them to the people coming from a different culture. This, he argued, would make the evaluation of adjustment process seriously biased.

In a similar fashion, based on the findings of a longitudinal study, Ward and her colleagues (1998) also rejected 'U-curve' theory. Looking at the sojourners' adjustment in a cross-cultural context their results did not confirm the U-curve theory propositions. Instead, their research demonstrated that their participants reported many psychological and socio-cultural problems at the initial stages of adjustment, but these problems seemed to have decreased as the length of stay increased in the host country. Likewise, results of an ethnographic study by Brown and Holloway (2008) confirmed that the longer the length of stay, the lesser the difficulty in adjusting to new host country.

Brown and Holloway (2008) suggested another model of adjustment by considering the duration of the stay of the international students in the host country. They referred to a source study which applied twin methods of interviewing and observation, and found that the international students were upset at the initial stages of their stay with high levels of stress. However, the stress levels were found to be minimal among these students in due course of time. Grounded on these observations Brown and Holloway conceptualised that the adjustment journey is an ever fluctuating process affecting the individuals throughout the period of their stay in the host culture as opposed to one 'U-curve' shaped process.

In light of the above views (Brown \& Holloway, 2008; Church, 1982; Ward et al., 1998) challenging the relevance of 'U-curve' theory, as well as the opposing view expressed by Black and Mendenhall (1991) calling any such rejections as 'premature', it would be stimulating to 
revisit this theoretical aspect of adjustment. The current empirical research focusing on the experiences of a particular group of international students (of Indian origin) would hope to contribute to the wider discussions on the adjustment process and relevance of ' $U$-curve' theory.

However, it must be remembered in this context that most of the above studies have viewed adjustment as a process and have used various 'models' to understand the adjustment process of sojourners. Such attempts have focused on different 'variables' influencing the adjustment process. For instance, psychological adjustment is associated with 'personality, social support and life changes' variables, and socio-cultural adjustment is connected with 'cultural distance, length of residence, language difficulty, amount of contact with host nationals and cultural knowledge' of an individual (Ward et al., 1998, p. 279; Ward \& Rana-Deuba, 1999, p. 424). While these variables appear to be exclusive to either psychological or socio-cultural adjustment, at times some variables could be found in all the three aspects of adjustment. For example, English language proficiency is a variable that influences psychological, sociocultural and academic adjustment process of an individual. Thus, drawing from the literature it can be argued that these 'variables' play a significant role in either advancing or hampering the adjustment process of an individual.

On the whole, adjustment of international students, although initially was viewed in terms of 'culture shock' or as a matter of English language proficiency (Adler, 1975; Church, 1982; Oberg, 1960), later studies have suggested a bigger picture in the transition of a sojourner (Black, Mendenhall, \& Oddou, 1991; Searle \& Ward, 1990; Ward \& Kennedy, 1993, 2001; Zhou et al., 2008). These later studies indicated that sojourner transition cannot simply be understood in terms of 'culture shock', but needs to be viewed as an 'adjustment' or 'adaptation' process involving psychological and socio-cultural aspects. However, later studies also pointed out that while discussing the adjustment process of international students, academic aspects should also be given due importance (Zhou et al., 2008; Zhou \& Todman, 2009). Thus, we now have a framework of observing and analysing the adjustment of an international student in all aspects of psychological, socio-cultural and academic contexts. 


\subsection{Different dimensions of adjustment challenges}

In a new host country, in a cross-cultural context, an individual may have a different experience because of the unfamiliar environment. Sometimes these experiences may constitute challenges affecting their positive adjustment to the conditions of the host country. Subsequently, it becomes priority for an individual to build or apply necessary strategies to cope with these challenges. Available studies provide an extensive coverage of both empirical and theoretical aspects of such cross-cultural adjustment issues.

\subsubsection{Challenges of psychological adjustment}

The psychological adjustment refers to the personal satisfaction or emotional well-being of an individual in a new host country (Searle \& Ward, 1990; Ward et al., 1998; Ward \& RanaDeuba, 1999). In the initial stages of adjustment a person experiences unfamiliar physical surroundings, cultural aspects, food habits and languages. These encounters with a different culture produce stress in an individual undergoing such transition (Ward et al., 2001). As international students relocate to a host country to pursue their studies, these experiences are common to them as well. As a result of this stress, an international student may display symptoms of passivity, vagueness, lack of interest in physical appearance, physical complaints, and struggle with communication (Khawaja \& Dempsey, 2007; Sandhu, 1994). Ward alluded to these symptoms at the initial stages of adjustment of an international student by the term 'foreign student syndrome' (Khawaja \& Dempsey, 2007, p. 14; Sandhu, 1994, p. 230).

This difficult and stressful time of adjustment often presents psychological challenges to international students. Sandhu (1994) after reviewing the past literature lists a few psychological challenges faced by international students in a foreign land. These include: depression, loneliness, feelings of helplessness, homesickness, high levels of anxiety and disorientation. Sandhu's list has been further expanded in other studies to include even more negative signs of adjustment. For instance, Ward and her colleagues highlighted that international students struggled in dealing with psychological problems such as 'fear, anxiety, anger and feeling of helplessness' (Ward et al., 1998, p. 278). Given that the above list of challenges is extensive and there is a vast literature available on each of them, it would be 
difficult to deal with each of these in detail in this review. So, one among the many psychological challenges, i.e. homesickness, will be taken as a sample to discuss their overall impacts on international students.

Poyrazli and Lopez (2007) in their exploratory study in the US define homesickness in terms of the reaction exhibited by individuals in the absence of the familiar people or surroundings. In addition, they note that homesickness could lead to loneliness, feelings of sadness, and difficulties in the adjustment process of an international student. Most importantly, they argue that homesickness affect an international student's behaviour, physical and psychological wellbeing (p. 264). Making similar observations, Thurber and Walton (2012) also note that staying away from home in a new surrounding could lead international students to experience stress and anxiety. They further argued that, at times, due to physical and psychological effects of this stress, it could result in individuals withdrawing from their course of study. Not only that, studies have indicated that homesickness can affect the academic performance of international students (Poyrazli \& Lopez, 2007; Tochkov, Levine, \& Sanaka, 2010). Given the negative impacts of homesickness on a sojourner, these studies have highlighted the need to set preventative strategies for a better psychological adjustment of international students in a new host environment.

Apart from homesickness, other challenges such as depression, loneliness, higher levels of anxiety, fear, anger, disorientation, and feelings of helplessness could equally impact an international student's psychological adjustment. Extensive attempts have been made to investigate a few common stressors that trigger these psychological maladjustment (Duru \& Poyrazli, 2007; Kwon, 2009; Poyrazli et al., 2004; Sandhu, 1994; Spencer-Oatey \& Xiong, 2006; Sümer, Poyrazli, \& Grahame, 2008; Wang \& Shan, 2007; Ward \& Kennedy, 1993; Zhang \& Goodson, 2011). Most of these studies indicate that the triggers behind the stressful situations are two common factors, a lack of language proficiency and friendships. For instance, based on their findings that language proficiency corresponds to psychological adjustment, these studies concluded that, an increased level of English proficiency decreases stress in the adjustment process (Duru \& Poyrazli, 2007; Poyrazli et al., 2004; Sümer et al., 2008; Zhang \& Goodson, 2011). Thus, English language proficiency is presented as a significant stressor affecting psychological adjustment of an international student in a foreign country. 
Similarly, lack of friendships significantly obstructs international students in their psychological adjustment. Ward and Kennedy (1993) suggested that co-national support networks helped sojourners to adjust better psychologically, while host national support networks result in better socio-cultural adaptation. This observation was further affirmed other studies indicating that increased social interactions relieved homesickness to a considerable extent (Kwon, 2009; Spencer-Oatey \& Xiong, 2006). Further evidence for the role of friendships is provided from the study of Wang and Shan (2007) conducted on Chinese postgraduate students in Australia. They identified that Chinese international students overcame the impact of loneliness and isolation by developing friendships, thus concluding that friendships can minimise the challenges of psychological adjustment.

On the whole, from the above discussion it is apparent that international students encounter several psychological challenges in the new host country. In addition, it was noted that stressors such as a lack of English language proficiency, and absence of friends could significantly affect their adjustment. In order to address such challenges, several studies have recommended coping strategies in the areas of individual abilities (English language proficiency) and social support (creating opportunities for friendships).

\subsubsection{Challenges of socio-cultural adjustment}

Socio-cultural adjustment refers to the international students' ability to adjust to their new socio-cultural environment (Searle \& Ward, 1990; Ward et al., 1998; Ward \& Rana-Deuba, 1999). The literature to this aspect considers the challenges encountered by an individual in learning/ recognising/ adapting to the social and cultural aspects of the new host country. While these challenges could vary, based on an individual's specific context as well as the context of the host country, this should not deter us from attempting to identify certain common factors encountered by the international students during their adjustment process. Such attempts could inform us of the challenges they encounter and coping strategies they deploy to adjust socioculturally in any given context. The identification of these factors through the available literature may help us further to address issues of cross-cultural transition of international students. 
In general, socio-cultural adaptation is associated with variables such as cultural knowledge, social skills, language proficiency, and length of stay in the host culture (Ward \& Kennedy, 1999). Lack of these abilities or skills could land an international student in social difficulties during the transitional phase. In such cases, a student makes special efforts to adjust positively in a new host country. Even though such efforts could head in several directions, their primary goal is usually to understand the cultural differences that mark their new host countries.

Choi's study (1997) on Korean students in Australian universities suggests that intercultural understanding is an important factor in improving the relational abilities of international students. Such an understanding further facilitates their relationships with their teachers and peers in the host country, thus enhancing their communication skills. Ultimately, the communicative abilities led to their effective adjustment in a cross-cultural atmosphere. Suggesting a reverse path, Andrade (2009) showed that the communicative ability of an individual helps that person to understand intercultural issues, leading to better adjustment. Consequently, this cross-cultural understanding helps a person to reduce the impacts of culture shock in a new place, and achieve an effective social adjustment along with academic success.

Not only intercultural understanding, but also the consequent knowledge about the new environment further enhance international student's appreciation of the new host country. As Brown and Holloway (2008) note in an article (based on the findings from an ethnographic study of the post-graduate students at a university in the South of England), cross-cultural knowledge enhances acceptance of and tolerance towards new cultures. The significance of their empirical findings lies in the fact that they point to an on-going transformation of the sojourners, as they accept a new culture due to the cross-cultural knowledge. Further, they even become 'human bridges' between cultures at a later stage.

A lack of the cross-cultural knowledge on the other hand can hinder the acceptance and tolerance of the new host culture, making socio-cultural adjustment even tougher. A study in Britain (looking at Malaysian students of two ethnic groups, Malay and Chinese) to examine the factors that affect socio-cultural adjustment concluded that the perception of cultural distance by international students has greater impact on their cross-cultural adjustment (Swami, 2009). The study pointed at two categories of students who perceived cultural distance differently. While, the Malay students who felt a huge culture gap showed poorer socio-cultural 
adjustment and low interactions with the host nationals, their Chinese counterparts had better socio-cultural adjustment and improved social interactions since they perceived less culture gap. These findings suggest that lesser the sense of cultural distance, greater the social interaction with host nationals and better the socio-cultural adjustment.

However, perceptions of individuals differ depending on different demographic variables such as age and gender of each individual. This phenomena was illustrated through the observations of the following studies which suggest the influence of demographic variables on the adjustment process. A survey of studies between 1978 and 1988 revealed that age and gender directly impacted psychosocial adjustment process (Cigularova, 2005). Contrary to this observation, Duru and Poyrazli (2007) in their study found no impact of these variables on the adjustment process. However, just a year later, one of the authors, Poyrazli, along with others observed that older students had higher levels of anxiety compared to younger students (Sümer et al., 2008). Poyrazli and her colleagues since agreed that demographic variables impact on the adjustment process. Similarly in an Australian study, Rosenthal, Russell, and Thomson (2008) too have noted that variables of age and gender have significant impact on the adjustment process. It is implicit that at the time of Church's (1982) review of the literature, 'gender' was not recognised as a variable associated with the adjustment process. Nevertheless, many later studies since have established its significance making demography an important variable to consider when discussing adjustment of a sojourner.

Apart from the intercultural understanding, knowledge, perceived distance and demographics, a person's adjustment depends on how well that person can socially interact in the new host country. Observing this phenomenon among international students, Ward and Rana (1999) on the basis of an empirical study proved that, contacts of international students with host nationals helped them to minimise their socio-cultural difficulties, thus allowing a faster adjustment. A later study conducted in France too confirmed this view, by stating that intimacy between domestic and international students helped international students to adapt better to the new socio-cultural environment (Brisset, Safdar, Lewis, \& Sabatier, 2010). Thus, the available studies affirm that friendship with domestic students is critical when adjusting in the new host country. 
While building relations with domestic students, what implicitly influence such contacts is the financial condition of the international students. This interesting relationship between adjustment and financial conditions was noted by Swami and his colleagues (2010) who observed that international students from high income families experienced better sociocultural adjustment. This would mean, a person from high income background would be at liberty to spend his/her financial resources to take part in events or sports or social activities to which their domestic peers might invite them, ultimately resulting in more social contacts.

Apart from the above factors, literature suggests that, perceived discrimination is a significant factor that influences socio-cultural adjustment (Campbell \& Jieping, 2006; Lee \& Opio, 2011; Novera, 2004; Poyrazli \& Grahame, 2007). The research indicates that international students often experience discrimination or they perceive themselves to be discriminated against (Lee \& Rice, 2007). It has been suggested that students from particular regions experience discrimination from their teachers and classmates at a significant level, and from other staff on a minor scale (Hanassab, 2006). Berno and Ward (2004) specifically noted that international students experienced different kinds of discrimination, and it was at a greater level in the case of Asian international students in New Zealand. Studies indicate that due to discriminatory practices or the perception of discrimination international students faced a substantial number of adjustment challenges (Mason, 2010, 2012). On the other hand, low levels of perceived discrimination could foster healthy attitudes towards the new host country, thus contributing to easier socio-cultural transition (Lee, 2010; Swami, 2009; Swami et al., 2010). So, in light of this discussion, it is evident that, international students often experience discrimination in the new host country. Such experiences could further affect their social skills and interactions.

Moreover, lack of special provisions related to food, religious practices, and differences in cultures could influence the socio-cultural adjustment of international students. A number of studies have indicated this problem facing international students due to non-availability of special provisions for religion, food and cultural habits (Alazzi \& Chiodo, 2006; Mehdizadeh $\&$ Scott, 2005; Novera, 2004). For example, Novera (2004) alerted to the fact that the religious custom of Muslims requires a person to pray five times a day, as well as need halal food. The study highlighted that since students spend most of the time outside of their accommodation, they found difficulties in observing these religious practices. In addition, Mehdizadeh and Scott (2005) pointed out that international students were brought up in the culture of their home 
country where their leisure time activities also conformed to their religious values. In a foreign country where they did not find it conducive to follow those religious practices, they faced pressures from their family members and this significantly hampered their socio-cultural transition.

Additionally, length of stay in a host culture is also considered an important factor impacting on socio-cultural adjustment of international students. Searle and Ward (1990) found no significance of this variable either in psychological or in socio-cultural adjustment. However, a number of studies since then have identified impacts of length of stay on socio-cultural adjustment (Junius, 1997; Ward \& Kennedy, 1999; Zhang \& Goodson, 2011). In a positive note, the review article by Araujo (2011) added that students with a longer length of stay were able to adjust to the host culture better than those who stayed for shorter period of time. While length of stay is a significant factor to consider in the socio-cultural adjustment, it is not a major challenge to overcome. In other words, unlike other variables, length of stay is not a variable that requires coping strategies. However, as a passive phenomenon, in a due course of time it helps an individual to acquire knowledge of the surroundings and develop social interactions, which ultimately is useful for positive socio-cultural adjustment.

Overall, the above discussion of literature on socio-cultural adjustment of international students highlights a few significant challenges. These challenges often arise because of lack of intercultural knowledge, skills and abilities of an individual to develop social interactions; lack of effective social support and English language proficiency. However it is well established that a better socio-cultural adaptation of an international student is possible especially through friendships with the host nationals. Some of the studies have suggested that international students should make individual efforts to develop relationships with domestic students, however, also asserting the need for support services when the students are found to be struggling in this respect. Besides this, it has also been noted that an individual's awareness of support services and the relevant information plays a crucial role in the socio-cultural adaptation. 


\subsubsection{Challenges of academic adjustment}

Generally, the primary goal of any international student (similar to any other student) is academic performance and achievement. However, from the above discussions it is apparent that international students face massive adjustment challenges in a new environment. So it can be assumed that in the academic front too international students encounter some challenges. Indeed, Chun and Poole (2009) emphasise that among all the reasons causing stress to international students, academic stress is the most important.

When Church (1982) explored the adjustment struggles of international students especially in academic matters, his findings indicated the following significant challenging areas: inadequate language skills, lack of understanding of different norms and procedures associated with the new academic system, lack of information and guidance on a different kind of educational system. According to Church, lack of language skills could lead to difficulties in understanding lectures as well as difficulties in written and oral communication necessary for pursuing studies. Likewise, lack of understanding of different norms and procedures in a new academic system refers to such aspects as a credit based study system, classroom protocols and teacher/student informality. In the same way, lack of information and guidance implies inadequate prior preparation, inadequate academic orientation and advice. In short, unfamiliarity with the language, norms and procedures of a new academic system, and information cause stress to international students.

Since Church's 1982 study various other studies at different times affirmed the significance of these three challenging areas, as well as added to this list a few more challenges that affect the academic adjustment of international students (Andrade, 2006b; Campbell \& Li, 2008; Mehdizadeh \& Scott, 2005). While Mehdizadeh and Scott (2005) further unpacked the issue of language proficiency to draw our attention to the problem of understanding different accents, Andrade (2006b) focused on problems of speaking and writing in academic contexts. Campbell and Li (2008) added a few more factors such as insufficient learning support, unfamiliar concepts of group discussions and assignments, difficulty in developing friendships with host nationals, and incorporation of cultural values in the educational practices, which affected the learning progression of international students. 
Similar to the issues associated with language proficiency, differences in academic cultures between the home and host countries of international students could also severely impact on international students' academic achievement. A review of literature by Ho, Holmes, and Cooper (2004) shows that different cultures are driven by unique features and objectives and will therefore have impacts on the learning, teaching and classroom interactions. Novera (2004) also makes a similar argument that academic cultural differences have greater impacts on the international students' learning process. The impacts of these differences are reflected in their learning styles, classroom interactions and student-teacher relationships. Holmes (2005) study on the Chinese students' communicative abilities at a New Zealand university revealed significant level of challenges experienced by them due to the cultural differences between their home country and host country. For example, Holmes, suggests that the inquiry based learning (which assumes interaction in the form of questions, expressing ideas and opinions) is a foreign concept to many students from Asia. The differences arise due to the participants' own familiar educational system in which teachers are viewed as the moral authority, a source of knowledge and therefore questioning a teacher is highly discouraged in Asian cultures. Unfortunately, these familiar views held by international students hinders their classroom participation and interaction with the teachers in the new host country.

Other than these issues, existing studies also suggest that international students face difficulties with a few academic conventions of the host country. For instance, Campbell and Li (2008) identified that even though students completed their undergraduate degrees in their home countries they were found to be lacking knowledge and understanding of certain academic conventions like independent learning, writing tasks, group discussions and group assignments. Essentially, the struggle of international students regarding the independent learning refers to taking up personal responsibility required to conduct and organise one's own research and study, particularly in an unmonitored context. Confirming this view, Eaves (2009) study stated that participants perceived that the learning process in England was stressful after reporting high level of differences in teaching learning process, independent learning, and assessment procedures.

In addition, Durkin (2008) explored another academic convention, i.e., the critical thinking approach valued in Western academic culture. Focusing on the East Asian postgraduate 
students, Durkin concluded that the participants of the study struggled with the concept of critical thinking, an important element of the western academic norms. The study uncovered that the memorisation approach, which international students were familiar with in their native educational systems was challenged in the western academic environment. Based on the study, Durkin commented that the academic conventions of a new host country could result in significant challenges to the international students influencing their academic adjustment and achievements.

To add further to the list of academic cultural variances, studies conducted on Indian international students in Australia and US found that one of the major gaps faced by their participants was in adjusting to an unfamiliar academic system in the host country (Kaur, 2007; Nayak \& Venkatraman, 2010). They particularly reported difficulties in writing assignments, as their familiar education system preferred yearly examinations over research oriented assignments.

Given, the stress international students' encounter in the new host countries, especially with the differences in academic cultures, easy access to necessary information could help them enormously. Although this aspect was emphasised in Lewthwaite (1996) study conducted in New Zealand way back in the mid-1990s, unfortunately, even a few recent studies have indicated that a similar lack of information on the academic norms and conventions has been prevalent, impeding the academic adjustment of international students in a host country (Andrade, 2006b; Campbell \& Li, 2008; Mehdizadeh \& Scott, 2005). To exemplify, Campbell and $\mathrm{Li}$ (2008) reported that the participants of their study revealed that due to lack of information a few students were unable to use the learning support or access their learning resources on the web. Consequently, this lack of information led them to underutilise the available institutional support, despite facing challenges academically.

\section{Overview of the discussion}

The above discussion on the adjustment of international students unravels different challenges faced by them in relation to their psychological, socio-cultural and academic adjustment. Each of these experiences were shaped by several contributing factors and when discussed together, we can identify a few common factors in all of them. These are: support services (S), English 
language proficiency (L), friendships (F), and information (I). In order to address their impacts, the studies recommended coping strategies, essentially involving enhancement of individual abilities as well as improvement of social support provisions from their institutions. So, any discussion on adjustment of international students needs to take into account two perspectives: the significant impacts of SLFI, and coping strategies to address these influences.

Other than this, the extant literature also discusses different opinions with regard to how an international student achieves better adjustment. While some studies highlighted the negative experiences (Lee \& Rice, 2007; Novera, 2004; Poyrazli \& Lopez, 2007; Tochkov et al., 2010), a few studies emphasised the positive adjustment experiences too (Swami et al., 2010; Wang $\&$ Shan, 2007). Some studies indicated that despite facing challenges students could be able to adjust well to the conditions (Campbell \& Li, 2008; Campbell, 2012; Ramsey, Ramsey, \& Mason, 2007). So, based on these studies it can be inferred that due to new environment and cultural differences, international students initially struggle to adjust, but in many cases in the long run they manage to cope and get a positive academic and life experience.

As for ability to cope with the conditions, while some studies have stressed the importance of an individual's own capabilities and self-responsibility in meeting the demands of SLFI (Andrade, 2006b; Duru \& Poyrazli, 2007; Rosenthal, Russell, \& Thomson, 2007; Wang \& Shan, 2007), other studies have emphasised the crucial importance of effective support services to assist these students (Eaves, 2009; Poyrazli et al., 2004; Sümer et al., 2008). To re-evaluate these assertions of the previous studies, a fresh study is required to re-examine the latest experiences and perspectives of international students in relation to their adjustment in a host country.

\subsection{Strategies to overcome adjustment challenges}

During the cross-cultural transition, to achieve better psychological and socio-cultural adjustment in a new environment, an individual needs to develop appropriate strategies and acquire culturally relevant social skills (Ward et al., 2001; Zhou et al., 2008, p. 69). Factors such as personality of an individual, and provision of social support contribute to a better psychological adjustment, particularly in cross-cultural transition contexts (Ward et al., 2001). 
Further, the provision of social support may include family members and friends. Similarly, the nature and extent of contacts with host nationals also strongly influence socio-cultural adaptation.

\subsubsection{Individual abilities}

In the midst of different psychological, socio-cultural, and academic challenges, it becomes essential for international students to focus on how they are coping with these challenges. If an individual is unable to properly deal with these challenges, that person may get adverse experiences. Additionally, many international students experience difficulties in the earlier stages of their stay, and therefore attention to coping mechanism becomes even more necessary. But such coping is dependent on personal abilities. The studies discussed below indicate the impacts and importance of individual abilities of a person in adjusting to the conditions of a host country.

Ward and Rana-Deuba (1999) emphasise that failure to cope with the psychological challenges lead to depression and mood disturbance in an individual. Similarly, Khawaja and Dempsey (2007, p. 22) explain how 'psychological distress' of sojourners is related to 'dysfunctional coping strategies' such as avoidance, repression, resignation, withdrawal. A dysfunctional coping strategy refers to a failure to perform the given tasks by the international students because they perceive those tasks as challenging or threatening. Moreover, Khawaja and Dempsey added that the dysfunctional coping strategy also negatively affects the overall health of an individual.

Other than these, psychological problems are said to be most serious at the initial stages of transition and are believed to dilute over a period of time (Cemalcilar \& Falbo, 2008; Ward et al., 1998). So, an individual should be equipped with coping strategies at an earlier stage in order to facilitate a quicker transition into the host culture. If this could be achieved it could promote a personal satisfaction or emotional well-being in an individual in a new host country, which essentially leads to positive psychological adjustment (Searle \& Ward, 1990; Ward et al., 1998; Ward \& Rana-Deuba, 1999). Therefore both the reasons suggest a need for actively exploring solutions in order to help international students to better adjust psychologically. 
In fact, the existing literature offers suggestions in this regard. For instance, Ward and Kennedy (1999, p. 661) suggested that psychological adjustment challenges are mostly affected by 'personality, life changes, coping styles and social support'. This observation involves mainly the characteristics of an individual such as personal flexibility and application of appropriate coping strategies in dealing with these psychological challenges. Different personality characteristics like extroversion, life changes, and locus of control ${ }^{1}$ were recognised as important variables that can facilitate the psychological adjustment of sojourners (Searle \& Ward, 1990; Ward \& Kennedy, 1993). Further, Ward and her colleagues (2004, p. 145) established that four out of big five personality features such as 'extraversion, agreeableness, conscientiousness, and neuroticism' were significantly associated with the cross-cultural adjustment process of sojourners. However, they could not establish any connectivity between adjustment and the other remaining personality factor of 'openness'. Nevertheless, Duru and Poyrazli (2007) in a later study explained that the openness feature was positively related to acculturative stress. Considering the importance of personality variables in the psychological adjustment process, it has been recommended that workshops be organised to educate international students on personality development matters (Cigularova, 2005).

Closely related to the 'personality' factors mentioned above, the characteristic of individual flexibility also marks easy psychological transition. Wang (2009) study conducted at American universities to explore the effects of resilience in international students revealed that, students with high levels of resilience had fewer adjustment issues. The study thus recognized individual 'resilience characteristics' (flexibility) as a crucial coping component in the adjustment process of international students.

Likewise, personal characteristics of self-efficacy or self-esteem have greater impacts on the adjustment process of international students. Van Oudenhoven and Van der Zee (2002) observed that 'Self-efficacy' of international students influenced their adjustment process in a different cultural environment. Similarly, another study of international students at a Malaysian university found that participants with high levels of self-efficacy had higher levels of

\footnotetext{
${ }^{1}$ Locus of control refers to the degree to which an individual sees himself in control of his life and the events that influence it.
} 
psychological adjustment (Mohd.Yusoff, 2011). The characteristic of 'self-efficacy' not only aided the psychological adjustment process, but also assisted in reducing any depressive symptoms experienced in the new host culture (Jackson, Ray, \& Bybell, 2013).

In such a way, when these individual personality traits are appropriately applied, the psychological transition of an international student becomes relatively easier. An illustrative case is aptly demonstrated in Wang and Shan (2007) study. Especially, the study notes that, international students overcome the major stressors of adjustment, viz, lack of language proficiency and lack of friendships, by making individual efforts such as watching TV, movies, listening to news, and developing friendships with the host nationals.

Similarly, other studies have indicated that, socio-cultural adjustment of an international student would be easier if the student had better social interactions with the host nationals (Brown, 2009; Rosenthal et al., 2007). However, a few studies have given credit to individual initiation for fostering relationships with host nationals (Duru \& Poyrazli, 2007; Rosenthal et al., 2007). Focusing on the Turkish international students in the US, Duru and Poyrazli (2007) highlighted that individual initiation depended entirely on the personality of a sojourner, in fact arguing that personality and social connectedness are related. In other words, the personal attitude of international students in terms of social connectedness helps in developing contacts with host nationals, which ultimately benefits them with a positive socio-cultural adjustment.

With regard to academic adjustment, Sandhu (1994) had noted that when a person experienced a feeling of satisfaction within oneself and with the surroundings, it contributed to their academic success in a cross-cultural environment. Andrade (2006b) in her review proposed three crucial aspects through which a proper academic adjustment of an international student could happen. The three aspects are: individual motivation, academic skills and familiarity with the circumstances, and external support over a period of time in the host country. Thus, individual abilities play a key role in academic adjustment as well.

To enhance individual abilities of international students, some studies have come forward with a suggestion to improve their English language proficiency. A few studies recommended increasing the required IELTS test score from the current norms in order to raise the proficiency levels (Holmes, 2005; Wang \& Shan, 2007). Therefore, there is an assumption that the 
increased levels of language proficiency, ultimately helps international students to acquire the required knowledge, be equipped with necessary skills, and develop contacts with the host nationals for better adjustment.

\subsubsection{English language proficiency}

English language proficiency plays a prominent role in adjusting academically, particularly in English speaking countries where international students not only undertake academic studies through the medium of English, but also interact with others using English language. It can therefore be assumed that those who do not struggle with English language would have a smoother academic adjustment, compared to those who struggle with it. A qualitative study by Novera (2004) examining the relationship between academic adjustment and English language proficiency among the Indonesian postgraduate students studying at different universities in Australia concluded that, participants perceived language difficulties as one of the significant barriers to academic adjustment. Novera highlighted that language difficulty became a major academic hindrance for all but two of the total twenty five post-graduate participants. As Poyrazli and Kavanaugh (2006) study determined, low levels of academic achievement corresponded with poor levels of English proficiency, thus, making English language proficiency a significant barrier encountered by international students, particularly from Asian countries, as they undertake their academic journeys.

In New Zealand too a study by Campbell and Li (2008) confirmed that the inadequate levels of English language abilities of Asian students hampered their growth in different academic areas. These areas include: communicative activities in their classrooms, understanding lectures, guidelines and procedures, writing tasks and developing social contacts with domestic students. This observation was further affirmed in other studies, which contended that language abilities have an enormous impact on the classroom participation of international students (Andrade, 2009; Henderson, 2009). English language proficiency is identified as a significant predictor of academic success of such students.

International students, especially at tertiary level in English speaking countries, are therefore required to provide evidence of English language proficiency through tests such as 
International English Language Testing System (IELTS) or the Test of English as a Foreign Language (TOEFL). The purpose of such a requirement is to ensure that international students have an acceptable level of English language competency to accomplish relevant academic tasks. Unfortunately, a few studies have reported that even though some international students scored high in the above tests, their language skills were not sufficient to ensure a better academic adjustment (Campbell \& Li, 2008; Holmes, 2005; Johnson, 2008; Wang \& Shan, 2007). For example, Wang and Shan (2007) suggested that many such students experienced learning shock due to a lack of language proficiency in both their verbal communication and academic writing. Ultimately, participants were deterred from actively engaging in classroom discussions, thereby affecting their academic achievement.

Even in the case of those who attended English as additional language (EAL) courses prior to their academic journey in the new host country, the results of such language proficiency tests were not helpful in facilitating academic success of international students. To illustrate this point, Johnson (2008) study discovered that even though EAL students proved their language proficiency through related language tests in a New Zealand university, they still struggled academically due to the complexities of the language involved in a classroom situation. Both these examples indicate that these international students may require additional knowledge beyond the testing of English language proficiency, such as usage of language in conversational contexts.

To summarise, it appears that students from non-English speaking countries may encounter academic challenges in classroom participation and discussions, and struggle with written and oral communication. This struggle is also noticed among those who undertake necessary language tests and courses. Therefore, either a lack of adequate level of English language proficiency, or a lack of self-confidence in terms of communicative ability may often result in academic underachievement of international students.

Apart from this, English language proficiency plays a crucial role in better psychological adjustment of international students. While, adequate language skill may reduces acculturative stress as it enables students to approach people for help or take part in classroom discussions (Duru \& Poyrazli, 2007), with lower levels of language proficiency being linked to depression and anxiety (Sümer et al., 2008). Similarly, the level of language proficiency is proportionally 
related to the international students' feelings of loneliness or active participation in the classrooms (Kwon, 2009). Kwon study stated that Asian students reported that their English language proficiency was lower compared to other students. It was therefore recommended that effective social support should be provided for such international students (Kwon, 2009; Sümer et al., 2008).

Likewise, low level of language proficiency corresponded to less number of social interactions with host nationals (Andrade, 2009; Campbell \& Jieping, 2006; Poyrazli \& Grahame, 2007). Better English language proficiency of Malaysian students in Britain helped them to adapt well, while students with low levels of language ability experienced poorer levels of socio-cultural adaptation (Swami et al., 2010). A similar situation prevails in New Zealand where a lack of language proficiency was found to be affecting international students in building relationships with the host community (Campbell \& Li, 2008; Campbell \& Jieping, 2006). This connection between language proficiency and social relationships was further stressed in literature on socio-cultural adjustment. A review article examining the predictors of psychosocial adjustment of international students in the US, surveyed sixty four studies published between 1990 and 2009 and found that English language proficiency was identified as a significant predictor for both psychological and socio-cultural adjustment (Zhang \& Goodson, 2011).

\subsubsection{Support services}

While a few studies have argued that better adjustment depends on individual abilities, other studies have pointed to what could be done to increase those abilities. For instance, Khawaja and Dempsey (2007) while maintaining the role of individual initiative to learn and use appropriate coping strategies to better adjust, also added that, support services in the educational institutions should focus on equipping the international students. This view is in accordance with an earlier study by Sandhu. As a remedy to minimise the psychological disturbance of international students, Sandhu (1994) proposed proactive and continuous counselling and support services from the educational providers. His reasoning seemed to be that, since the individual student struggles adopting to the host culture, the host culture therefore, should take responsibility in empowering that student through support provisions to experience positive psychological adjustment. 
Additionally, when an individual lacks the ability to initiate contacts with host nationals, studies recommend that such a person be offered support by the educational institutions. Otherwise, the socio-cultural adjustment would significantly be hampered due to a lack of these skills. So, studies recommend fostering of these skills through support services. For instance, Cigularova (2005) recommended that the relationships between international students and host nationals may be developed through specialised programmes such as conducting culture training in a diverse cultural environment. Furthermore, Ward (2006) argued that since a desire for mutual social connectedness exists in both international students and their domestic peers, the frequency of contacts between them should be increased. To achieve this objective, Ward recommended interventionist strategies from the educational institutions. Subsequently, some studies have endorsed such specific institutional programmes such as 'The Massey Kiwi Friend Programme' (Ramsey et al., 2007), or the 'buddy project' at a university in New Zealand (Campbell, 2012), which promoted social contacts and cross-cultural knowledge among international students. The programmes were said to be effective in achieving positive results.

Regarding academic culture and conventions, the available literature also offers a few recommendations. Andrade (2006b) suggested that host countries should understand the academic challenges of international students and need to provide them adequate support to enhance their knowledge and skills. Making a similar argument Poyrazli and Kavanaugh (2006) argued for helping international students by providing opportunities to improve their language skills, which may promote interactions as well with domestic students. McClure (2007) suggested that prospective international students be made aware of the relevant academic aspects such as self-study and the nature of educational system of the new host country. Mehdizadeh and Scott (2005) insisted on the need to make international students aware of the difference between dependent and independent learning procedures. Taking this argument further, Eaves (2009) noted that international students should be assisted with extra support in order to enable them to meet the academic requirements of the host country.

Extending the view, Campbell and Li (2008) proposed that host institutions should not leave the responsibility totally on the international students. According to them, this responsibility would primarily be on the staff, for providing appropriate support to equip international students with the required knowledge on academic conventions and practices. Such a view seemed to gain support as a few other studies indicated need for teachers to be aware of the 
changes required in communicative styles, and learning practices while dealing with students from diverse cultures (Johnson, 2008; Millar, 2009; Soosay, 2009; Turner, 2009). A recent study recommended that the teachers need to be equipped with cross-cultural understanding while involved in teaching in cross-cultural environment (Vu \& Doyle, 2014). Johnson (2008) urged the educational institutions to realise the impacts of cultural differences on academic integration of international students. Considering the need, a prior study in New Zealand had contended that it was necessary to provide proactive support to international students by identifying their problems in the academic area instead of providing them reactive support at the time of crisis (Butcher \& McGrath, 2004). Even though at times, learning approaches of international students might vary, sufficient support together with proper teaching environment can lead to better adjustment to the conditions (Baker, Isaac, Li, \& Marshall, 2005).

While having such support services can be of immense help to an international student, it is also vital to provide necessary information regarding the availability of such support, so that students can make use of these services. International students have been found to be underutilising support services and facilities in their institutions (Ward, 2001). This limited usage of the available services seems to be a common feature everywhere. For example, an Australian study too revealed that despite facing challenges in their initial adjustment period, international students were not aware that they could use the support services in their university for sorting out their personal issues (Ang \& Liamputtong, 2008). Further, the study exposed that lack of knowledge led the international students to view such services as only for those with psychological illnesses. Hence, appropriate information regarding the availability of support services has been deemed essential to enable students to utilise them effectively.

The need to inform international students about the availability of support services was also underlined in other studies (Arambewela \& Maringe, 2010; Campbell \& Li, 2008; McKinlay, Pattison, \& Gross, 1996; Sawir et al., 2009a, 2009b). Some studies have recommended a continuous flow of information throughout the entire period of study of an international student (Chun \& Poole, 2009; McKinlay et al., 1996; Zhang \& Goodson, 2011). Mehdizadeh and Scott (2005) maintain that educational institutions should take responsibility to organise regular trainings and workshops in order to provide information related to socio-cultural differences and academic expectations of the host country, as well as support available to cope with them. 
Such a continuous stream of information can result in proper utilization of the resources by international students. While some studies suggest supplying pre-entry information (Lewthwaite, 1996; McClure, 2007), others (Berno \& Ward, 2004) recommend that information should be provided at both pre-entry and post-entry stages. Again, a recent study which aimed to evaluate the levels of difficulty in obtaining relevant information by the international students in New Zealand, reported that especially, Bachelor and doctoral degree international students experienced difficulty in finding necessary information at the pre-entry stage (Bethel \& Ward, 2014).

It is expected that when international students are made aware of support services, it could result in proper utilization of the resources. As Andrade (2006a) demonstrates in an empirical study, when students are informed through a seminar of different regulations and procedures of the US education system, of available resources in the university, and of cultural aspects of the host country, it leads to positive results. This means, the relevant information passed on to students in the seminar is regarded as very helpful, thereby resulting in utilization of the resources when needed.

The available literature also suggests the ways in which such support services should function to ensure even better utilization. For instance, Pang (2012) proposed a comprehensive dynamic learner-centred conceptual organiser, informed by the author's own experiences as learning advisor, and supplemented by certain applied theoretical principles. This concept was termed 'developmental advising', and it was explained with an illustration of how students could be helped with the particular academic skill of 'referencing', required in the western educational system. Pang suggests, the knowledge of how particular academic skill works will create a positive approach to learning and a deeper understanding of that skill. Ultimately, this understanding helps a student to apply it at a multilevel and multicultural context.

When students are informed and provided with ample assistance, it could lead to better utilisation of support services in their institutions. Based on the observations of a review study, Olivas and Li (2006) reported that, when the support services were better utilized by international students it resulted in better adjustment prospects. Hence, they recommended that a successful strategy for academic adjustment of international students, should focus on both proper utilization of available support services as well as individual abilities - not one or the 
other. Therefore, it becomes essential not to underestimate the value of information and services for better academic adjustment of international students.

Apart from this, there is also an argument for providing information to prospective international students at an early stage of their decision making about a study destination. At this point, the educational agents play a key role in offering services in the origin country as a representative of the host country educational institutions. Generally, agents focus on smooth and quick process of visa approval for gaining good will of the customers and to increase their business (Bandyopadhyay, 2010). The priorities of the agents are of course their business interests rather than providing necessary information to the prospective students. The National Survey (Deloitte, 2008) in New Zealand specially found that university students were not satisfied with the services of educational agents. However, a later national survey in 2011, indicated a change of trend, wherein the majority of the participants reported satisfaction with the services offered by educational agents, while a small, though not insubstantial, percentage (15\%) expressed dissatisfaction (Business and Economic Research Limited, 2013). Moreover, the latter report signposted that Indian students, unlike Chinese students, generally had a tendency to make use of the services of the educational agents while making applications. This highlights the need for improving services of the educational agents at an earlier stage in order to equip prospective international students with necessary information.

To summarise the discussion above, challenges of academic and social life are inevitable for international students whenever they enter a new host country for study purposes. The related literature has highlighted these challenges, which are mainly because of inadequate levels of English language proficiency, unfamiliar educational system, and inadequate levels of information and social support. Simultaneously, these studies while recognising the importance of individual abilities in overcoming these academic and social life challenges, have also recommended necessary assistance to be provided to the international students through support services of the educational institutions. Specifically, the provision of necessary information and assistance in improving their knowledge and skills is anticipated to facilitate their better adjustment to the new environment of a host country. 


\subsection{Experiences of international students in New Zealand}

Different monitoring policies are being implemented in different countries by their respective governments in relation to international students. In New Zealand, the government in 2002 introduced a regulatory enactment called the Code of Practice for the Pastoral Care of international students. The Code empowers the Government to monitor, regulate, audit, set standards and check quality of education imparted by the educational institutions (Lewis, 2005). The code was designed to push export education industry towards adhering to global standards in order to remain internationally competitive. However, it was revealed that many of the student participants in a study were unaware of their rights under the provisions of the Code, and further, they were even unaware that their universities were bound by the regulations of the Code (Sawir et al., 2009b). Based on the observations, the study opined that in the absence of information these students neither claimed their rights under the code nor used the intended pastoral support. Butcher and McGrath (2004) study therefore insisted on proactive support instead of reactive support to the international students in meeting their academic and social needs in New Zealand. Later, the national survey of 2007 on the experiences of international students in New Zealand too, having noticed a significant percentage of respondents (42\%) rated the overall quality of services in their educational institutions as either average or poor, and recommended evaluatory measures by the institutions on the available resources meant for such students (Deloitte, 2008).

However, it has also been reported that among the new players in recent decades entering the export education market, New Zealand has registered significant performance in increasing its share of international students at tertiary level (OECD, 2013; Verbik \& Lasanowski, 2007). In light of the above observations it can be argued that improved facilities and services for international students will be further required in the educational institutions of New Zealand for them to remain competitive. Hence, this study proposes to explore the latest conditions in New Zealand, through the experiences and perceptions of a particular group of current international students.

In this respect the current research will take into account the push-pull factors that influence international students making a decision on a study destination. Generally, the trend identified is the personal positive attitude of individuals to study abroad having the greatest impact on 
their decision-making process (Chen \& Zimitat, 2006; Cubillo, Sánchez, \& Cerviño, 2006). Similarly, information through a 'word of mouth' is also an important factor for prospective international students in making a decision on study destination (Bandyopadhyay, 2010).

However, in the case of New Zealand, the available literature indicates that along with some general considerations, specific factors also influence the students' decisions. These factors (an English speaking environment, low tuition fees, quality education, and safe environment of the country) influence the decision making process of international students choosing to study in New Zealand (Deloitte, 2008; Ward \& Masgoret, 2004). Along with safe environment, a recent survey highlighted the quality of teaching, image of the institution, reputation and value of qualifications as influencing students' decisions (Business and Economic Research Limited, 2013). Besides these factors, the influence of parents anticipating better employment prospects and quality of life also guide their decisions (Ho et al., 2007). The proposed study seeks to further explore the reasons, hoping to add to the list of considerations that may influence student decisions in selecting New Zealand as their study destination.

\subsection{Asian international students in New Zealand}

Among the community of international students, students from the Asian region have been particularly identified to be facing numerous challenges while adjusting to the conditions of the host country. A few studies have indicated that these challenges are because of huge differences in the cultures and academic expectations, particularly in an English speaking environment (Berno \& Ward, 2004; Butcher \& McGrath, 2004; Campbell \& Li, 2008; Campbell \& Jieping, 2006; Ho et al., 2004; Ho et al., 2007; Holmes, 2005). Consequently, these differences influence the academic achievement of the Asian students. While the review study by Ho et al. (2004) described these challenges to be natural and inevitable, other studies have highlighted the seriousness of their impacts on Asian students and their academic achievements.

Based on the observations on adjustment experiences and adaptation processes of Asian students in New Zealand, Berno and Ward (2004) concluded that the experiences of the students under-met their expectations in both academic and social life contexts, and 
recommended necessary social support measures from the educational institutions. Another exclusive study on the Chinese students in New Zealand indicated that the participants experienced difficulties due to their inadequate levels of English language proficiency, and because they were coming from different academic culture and educational practices (Ho et al., 2007). Further, this study indicated that tertiary level students experienced more learning difficulties. Other than these, participants reported dissatisfaction with accommodation facilities. A majority were upset with the feelings of isolation and loneliness in the initial months of their stay, and mostly depended on co-national support networks instead of formal support from their institutions. Based on these observations, this study recommended several measures to enhance the quality of living and learning experiences of Chinese students.

On the contrary, Campbell and Li (2008) study on Asian students in a New Zealand university found that the participants despite experiencing difficulties, reported positive learning experiences. However, the participants also felt that their lack of English language proficiency was a barrier preventing their learning activity. In the middle of a different educational culture and practices, students had insufficient learning support. A majority of the participants reported difficulty in developing friendships with the host nationals.

In general, students from Asian countries are identified as with poorer levels of English language proficiency which hinders their adjustment and academic achievement (Campbell \& Li, 2008; Campbell \& Jieping, 2006; Holmes, 2005; Johnson, 2008). Noticing the serious impacts of poorer levels of English language proficiency on the adjustment process international students these studies recommended better social support provisions. While the educational institutions insist on English language proficiency to be proved through language tests before enrolling them, we have already noted that they are not sufficient indicators of language skills.

Besides these, some studies on Asian students have underlined the value of contacts with the host nationals and consequent impacts on their socio-cultural adjustment (Berno \& Ward, 2004; Campbell \& Li, 2008; Ho et al., 2007). At the same time, it was found that making friendships with domestic community and conversing with them in daily life contexts was also a huge problem for many international students (Campbell \& Jieping, 2006). Based on their 
observations these studies recommended interventionist strategies from the educational institutions to establish contacts between the international students and the host nationals.

Moreover, international students from the Asian region have alleged experiences of discrimination at a higher level than other groups of students affecting their smoother transition to the conditions of the host country (Berno \& Ward, 2004; Campbell \& Jieping, 2006). The observations of these studies indicated that such perceptions of discrimination result in negative impacts on their otherwise positive adjustment in a host country. Unfortunately, some incidents of discrimination were found to be specially targeting students from a particular region or country. Noticing the adverse impacts of such incidents on international students, studies have suggested that specific measures should be taken for establishing healthier environment in the educational institutions.

Furthermore, providing effective support to Asian students was felt very appropriate by the studies for enhancing their knowledge and skills to cope with their adjustment challenges. The key role of support services in organising specialised programmes for Asian students to develop contacts with the local communities was very much recognised. At the same time, studies have signposted that despite experiencing challenges, Asian international students often do not sufficiently utilise support services in their institutions (Campbell \& Li, 2008; Ward, 2001). This is because either they are unaware of the services or think that the supporting staff cannot help them as their problems relate to cross-cultural context.

Altogether, the literature has indicated that among all the international students, those from the Asian region experience more difficulties while adjusting to the conditions of a host country. On the other hand, the statistics also indicate that among the international students enrolled at tertiary level worldwide, the majority are from Asian countries, such as China and India as indicated earlier. This situation indicates a need for further studies into their current academic and social life conditions, that can help framing or amending supportive programmes by educational institutions for the burgeoning number of Asian international students. Hence, this study intends to explore the experiences of a particular group of Asian international students studying in New Zealand. 


\subsection{Need for a study on Indian international students in New Zealand}

Since New Zealand has international students from several source countries, this study is focussed on a particular international student community from one source country, that is, India. This group has been selected after due consideration of a set of reasons. First of all, a report by the International Division, Ministry of Education in New Zealand, published in 2011, stated that, among all the countries from where international students were enrolling in New Zealand between 2004 and 2010, the top five source countries or regions were from the continent of Asia (Ministry of Education, 2011a, p. 4). These included: China, South Korea, Japan, India, and South East Asia (Thailand, Malaysia, Vietnam, Cambodia, Singapore, Philippines, \& Indonesia). This interesting trend further compels us to explore the adjustment experiences of international students arriving from Asian countries.

However, instead of studying students from multiple countries from a culturally diverse region like Asia, it is preferable to focus on the experiences of a specific group of students from a single source country. The national survey of 2007 in New Zealand indicated that studies focused on particular nationalities might help us better understand specific needs and expectations of such groups and better facilitate support services for them (Deloitte, 2008). Equally, it has been argued that there is a need for ethnicity based studies to meet the demands of establishing a culturally competent environment in higher educational institutions (Chun \& Poole, 2009).

As mentioned above, studies on a particular group of students may uncover the specific features of that particular group. For instance, it has been identified that more than $90 \%$ of Indian students enrol at the tertiary level, and they tend to prefer long-term and higher value courses compared to the general trend among international students (Ministry of Education, 2007). Similarly, while the number of international student enrolments in New Zealand decreased from other source countries such as China and Japan between the years 2006 and 2010, the trend showed increase of enrolments from India (Ministry of Education, 2011a). Furthermore, in the national survey of 2011, it was specifically pointed out that Indian students in their home country mostly depended on the services of educational agents while making applications to the educational institutions, unlike Chinese international students (Business and Economic 
Research Limited, 2013). Similarly, a recent article highlighted that Indian students' mobility depends on economic potentiality of their families and social conditions existed within India (Gopinath, 2015). While exploring the experiences of this particular group of students, specific reasons for such preferences and traits may be explored.

Additionally, among the international students in New Zealand from different Asian countries, those arriving from India have shown to be ever increasing in number, registering the highest growth of 346\% between 2006 and 2010 (Ministry of Education, 2011a). Nevertheless, when compared to the number of Indian students opting for the US for educational opportunities in higher education, the number of students arriving in New Zealand is very less (Bandyopadhyay, 2013). This article illustrated that in 2011, out of the total of 100,270 Indian students left their country pursuing higher educational opportunities in other countries, only 12,300 choose New Zealand as their study destination indicating a vast scope to capture the potential Indian market for international students.

Moreover, as a part of collaborative initiative in the field of education, an 'India New Zealand Education Council' was set up which is annually funded by both the governments, to promote research and higher education (Bandyopadhyay, 2013). India is considered as one of the important source countries for international students in the university sector and, particularly for PhD enrolments in New Zealand (Ministry of Education, 2012). However, the statistics indicate that out of the total enrolments of Indian students in New Zealand in 2010, only 11\% enrolled in the universities and a majority of students enrolled in other tertiary level institutions such as private training institutions (63\%), and polytechnics (26\%) (Ministry of Education, 2011a). While an encouraging environment exist for promoting research, the reasons for low enrolments in the universities needs to be explored.

Most importantly, although Indian students have been coming to New Zealand since 1951 (Tarling, 2004), published literature on international education in New Zealand indicates that no exclusive studies have been undertaken on this group. By contrast, exclusive studies are available on other Asian international student groups in New Zealand such as the Chinese and the Vietnamese (Campbell \& Jieping, 2006; Ho et al., 2007; Holmes, 2005; Vu \& Doyle, 2014). At the same time, available studies on Indian students in other countries have featured that 
these students face a different set of adjustment difficulties in their academic life (e.g., unfamiliar academic conventions) (Kaur, 2007; Nayak \& Venkatraman, 2010), and social life (e.g., perceived discrimination) (Mason, 2010, 2012). An ethnographic study (Baas, 2010) on Indian students in Australia indicated that in addition to their immediate academic goals, the students had longer term aspirations such as employment in the host country and eventually immigration. Such findings certainly warrants a study to find out what their counterparts in New Zealand want to achieve.

On the other hand, research undertaken in the UK to predict trends of postgraduate student mobility in the world up to the year 2024, projected that among the potential source countries for international students, India will be sending the highest number of tertiary students even superseding China (MacGregor, 2014). The increasing number of Indian students in different host countries warrants a study of their specific experiences or challenges they face in adjusting to the conditions of their host countries. Ultimately, the strategies applied by these students may point to helpful solutions that other prospective international students may use to overcome their adjustment challenges.

The increasing numbers of international students and student mobility in tertiary sector worldwide have given impetus to probe the experiences or issues related to such students in the higher education sector (Chan, 2012; Dolby \& Rahman, 2008). Within this global context, New Zealand government has put strategic emphasis on promoting international education as a means of strengthening its tertiary education system (Bandyopadhyay, 2013; Ministry of Education, 2007). As a part of this strategy, New Zealand has focussed on establishing education related contacts with India at tertiary level. In view of that, a study of the experiences of Indian students at tertiary level may be helpful in developing and maintaining healthy relations between India and New Zealand. Considering all the above views, this study has been limited to the Indian students pursuing their studies at tertiary level, particularly in universities, covering all the eight universities in New Zealand.

The above discussion suggests a gap in the literature on international student experiences in New Zealand related to the adjustment experiences of Indian international students. Accordingly, this study is intended to examine the experiences of Indian international students enrolled in New Zealand universities, particularly in relation to their academic and social life, 
including their language experiences. Moreover, considering the significance of support services as indicated in the extant literature, it also proposes to explore the perceptions of these students on the use and effectiveness of support services in their universities. Hence, this research has been conducted with the following research questions:

RQ1: How do Indian international students in New Zealand universities adjust to academic and social life in their new host country?

RQ2: How do Indian international students studying in New Zealand universities perceive and use support services? 


\section{Chapter 3 \\ Research Design}

\subsection{Overview}

The objective of this study is to examine the adjustment experiences of Indian international students related to their academic and social life conditions in New Zealand, including the challenges they faced and strategies they applied to overcome them. Further, this study proposes to collect data on the usage of the support services by the participants along with their perceptions on the effectiveness of those services. For this purpose, two research questions were framed. The initial question sought information on their experiences of adjustment in their academic and social lives to the conditions prevailing in New Zealand, and the other question was about their perceptions on the use and effectiveness of support services in their universities. This chapter outlines the research approach employed in this study, and how different procedures were adopted to carry it out. Hence, the following sections will provide details of the research planning, data collection and analysis, interpretation procedures, along with a note about the ethical considerations how they were addressed in this study.

\subsection{Designing a research approach for this study}

The designing of a specific research project requires the researcher to consider different strategies of investigation, such as quantitative, qualitative or mixed method approaches. (Creswell, 2003, 2009). After considering these three methods, a mixed methods approach has been chosen to conduct this study. The proposed study thus intends to use both quantitative and qualitative approaches, use both close-ended and open-ended questions for collecting data, and plans to collect both numerical and descriptive data for this study. Further, Tashakkori and Creswell (2007b) by identifying seven indicators such as two types of questions, or sampling techniques suggested that if a study involves at least one of these indicators it can be designated as a mixed method approach (Tashakkori \& Creswell, 2007b). 
Three essential principles should be considered by a researcher while choosing a research design. Firstly, the researcher should assess which research approach would aptly and efficiently suit the research problem. Secondly, the researcher should also consider whether he/she is equipped with the knowledge and training required for conducting research with the selected research approach. Finally, the researcher should also think about the general consideration of their advisers and audience in selecting a research approach (Creswell, 2003, 2009).

Consequently, a careful assessment has been made on whether or not applying a mixed methods approach would be appropriate for this study. For this purpose, several factors were taken into account. It is, first of all, well-known that a combination of both quantitative and qualitative approaches can restrict the weaknesses in each of these, and enable collection of valid and indepth information (Ivankova, Creswell, \& Stick, 2006). Similarly, it has been found that the use of mixed methods approach not only helps better understanding of the complexity of a social inquiry, but it also enhances the 'validity and reliability' of the research study (Greene, 2007). Other than these, it should also be noted that 'using of both qualitative and quantitative approaches to explain and explore a social phenomenon is gaining momentum as a third methodology' (Hall \& Howard, 2008, p. 248). The current trend in social science research is indicating that the use of mixed methods approach is well accepted and widely used (Ivankova et al., 2006; Johnson, Onwuegbuzie, \& Turner, 2007). Most importantly, in case of a situation where each of the research approaches is not believed to be fully helpful in understanding a research problem, mixed methods approach appears to be the most appropriate approach to be applied (Creswell, 2010).

Further, it has been suggested that if an intended study seeks to achieve more than two objectives among the five listed by Leech and Onwuegbuzie, such as 'exploration, description, explanation, prediction, and influence', a mixed methods option may be considered as appropriate (Leech \& Onwuegbuzie, 2010, p. 62). This study has dual objectives: the primary objective is to explore the adjustment experiences of Indian international students studying in New Zealand universities, while the secondary objective is to consider their perceptions on the use and effectiveness of support services in their universities. On the whole, to gain from the strengths of both quantitative and qualitative approaches, and to minimise their weaknesses when used independently, this study uses a mixed methods approach. 
To fulfil the second requirement, the researcher has become familiar with the concepts and procedures of both quantitative and qualitative approaches. The quest for knowledge has led the researcher to understand different philosophical traditions of how knowledge is derived, theoretical world views based on these philosophical traditions, different research approaches and selection criteria for a particular research design. Besides these, the researcher acquired knowledge of practical skills to perform different procedures associated with collecting, analysing, interpreting the data, and reporting the study in a systematic manner. Other than these, the researcher has also the necessary cultural knowledge and skills for designing and conducting online surveys, and of using digital devices for recording and transcribing individual interviews along with a few techniques to be used while interviewing (e.g., noting down the non-verbal communication of the participants). Having acquired necessary levels of knowledge on different theoretical components and different practical aspects associated with both the approaches, the researcher has gained confidence to make use of this knowledge and skills and employ them during the actual process of the study.

In accomplishing the third principle, the researcher approached the two supervisors of this study and discussed in detail different approaches available that might be considered for this study. The matters associated with the time period in conducting research, assessment of risk involved in undertaking different research approaches, and the advantages and disadvantages of opting for individual approaches were thoroughly discussed. After evaluating these multiple factors the researcher and the supervisors were well convinced to apply a mixed methods approach for this study. After selecting the particular research approach, the attention of the researcher was turned to the selection of a suitable research model from among the different options available.

\subsection{Selection of a research model}

Four important influential factors that affect the research process need to be considered while selecting a model for a study using mixed methods approach (Creswell, 2009). They are: period of time required for data collection for each approach either in separate phases or collected simultaneously; ratio of priorities for the data collected in each method whether equally or proportionately given weightage; the mixing of data from each method whether at data 
collection phase or at data analysis phase or at data interpretation stage; and any theoretical principles guiding the research design. Alternatively, it is also suggested that once a research approach for a study is selected, the researcher will be free to choose any kind of research model in that particular approach (Johnson \& Christensen, 2012).

However, after considering the factors mentioned above, out of the six types suggested by Creswell (2009), a sequential design was selected for this study. It was selected for the following reasons. Firstly, this model suggests collection and analysis of quantitative data in the first phase, and the subsequent collection of qualitative data to be based on the preliminary observations on the quantitative data. In this model the initial quantitative data determine the second phase of qualitative data collection, thus, priority is given to the initial quantitative study. When the data collection process and analysis are conducted separately and mixed at the stage of interpretation to attain precise understanding of the research issues, this design can be advantageous. This model has been referred to as a straightforward approach by several studies as it involves separate, clear and easy steps in the implementation process (Creswell, 2009; Creswell \& Plano Clark, 2007; Ivankova et al., 2006; Plano Clark \& Creswell, 2008; Tashakkori \& Teddlie, 2003). Finally, when the intention of a study is 'development', which means findings of the initial study is to be used in the second phase of study for in-depth information, sequential design is suggested to be a better option (Onwuegbuzie \& Collins, 2007).

This model involves two separate phases of data collection which take a long period of time; but still it has been considered most appropriate for this study as it fulfils all its requirements. By following this method, initially a survey questionnaire was used to collect quantitative data, and in the later stage individual interviews were conducted to generate the qualitative data. The specific reasons for opting for a questionnaire for collecting quantitative data and individual interviews for generating qualitative data will be explained later (while explaining the research instruments). Overall, the responses of participants in the survey questionnaire were expected to frame broader perspectives or identify general trends on the research issues. On the other hand, data gathered through individual interviews were expected to provide detailed and indepth information on the same research issues. As the quantitative data were collected initially and they determined the collection of qualitative data, the initial quantitative approach was given due weightage. Further, even though both the quantitative and qualitative approaches of 
this study were conducted at different stages, and data analysis was carried out separately, both the data sets were connected together at the stage of interpretation of the findings.

Furthermore, in this sequential design two forms of mixing are suggested, connecting both the quantitative and qualitative approaches (Ivankova et al., 2006). Firstly, the same participants should be used in both the approaches. It is recommended that the participants from the earlier survey be used in subsequent interviews as they have some understanding of the research issues of the study (Creswell \& Plano Clark, 2007). Secondly, both the approaches may be linked by integrating the results of individual approaches at the point of interpretation. This study has applied both the forms suggested in the existing studies while connecting the quantitative and qualitative approaches. Interviewees of the second phase of study have been chosen from the participants of the initial survey. Besides this, the outcomes of analyses of each set of data were integrated while interpreting the findings of this research.

\subsection{Research planning of this study}

Research planning of this study broadly involved selection of sampling techniques, and determining strategies of inquiry by using the research instruments. It has been suggested that appropriate care applied in sampling strategies will bring validity to the collected data (Creswell, 2009). The strategies of inquiry involves the determination of selection criteria, and the preparation of research instruments for collecting data from the participants in each phase of the study.

\subsubsection{Sampling techniques}

Purposeful sampling strategies were applied for both the initial quantitative and later qualitative phases of this study. Usually, quantitative and qualitative studies involve probability (random) and non-probability (non-random) procedures respectively (Creswell, 2009; Teddlie \& Yu, 2007). However, it was indicated that a mixed methods researcher may employ a non-random (purposeful) sampling for both the phases of study based on the objectives of the study (Onwuegbuzie \& Collins, 2007). Similarly, if a sampling technique is able to answer the 
research questions efficiently, a researcher may employ either a probability or a non-probability sampling technique exclusively for collecting both the quantitative and qualitative data (Teddlie \& Yu, 2007). Accordingly, for this study among the purposive sampling techniques, a 'snowball sampling technique' was used in the first phase of quantitative study, as it was facilitated by help from the participants of the study who introduced the researcher to new participants. In the second phase of study, individual interviews were conducted by selecting the interviewees from among the participants of the initial survey, according to the suitability criteria, such as gender and level of study by using 'convenience sampling technique'.

\section{Sample size}

The sample size for this study was set after considering the significance of factors like yearly enrolment numbers of Indian students in tertiary educational institutions in New Zealand. The target set for initial quantitative approach was at least 100 completed surveys. It was believed that this quantity of data would be sufficient to make reasonable interpretations and to achieve valid conclusions about the study. It is indicated in the available literature that a minimum of 100 units are needed when studying different behavioural patterns of individuals (Onwuegbuzie \& Collins, 2007). In the case of using purposive sampling techniques, though it is recommended that thirty samples or less will be necessary, for a quantitative approach it should relate to the population size to represent its characteristics appropriately (Teddlie \& Yu, 2007).

This study was restricted to focus on Indian international students studying in universities in New Zealand for valid reasons. While looking at the distribution of the random survey samples of the national survey of 2007 and considering the enrolment patterns of international students in New Zealand at different levels (schools, polytechnics, universities), the university students were recognised as a major group (42\%) across the sectors (Deloitte, 2008). Apart from this, India has been identified as an important source country for international students for the university sector, particularly for $\mathrm{PhD}$ enrolments in New Zealand (Ministry of Education, 2012). Another significant factor given due importance was that a majority of the Indian international students were found to be preferring particularly higher value courses at tertiary level (Ministry of Education, 2007). Interestingly, although the government identifies India as a major potential source country for university enrolments, the enrolment pattern of Indian 
students in New Zealand universities is comparatively low than the enrolments taking place in other tertiary institutions such as Polytechnics, and private institutions. For instance, in 2010 out of the total of 11,597 of Indian students enrolled in New Zealand, only $11 \%$ enrolled in universities, while the other $89 \%$ enrolled in other tertiary institutions (Ministry of Education, 2011a). Even in the successive years of 2011 and 2012, this percentage of Indian student enrolments in the universities oscillated around nine percent, while the enrolments in other tertiary institutions rose to $91 \%$ (Ministry of Education, 2013). This situation warrants the need to explore the factors surrounding the Indian students, particularly those enrolled at universities in New Zealand. Thus, with the advice of the supervisors, this study was restricted to a single sector of university students studying in New Zealand. Nevertheless, the scope of the study was extended to all the eight universities in New Zealand.

With regard to the sample size for the qualitative phase of study, considering the guidelines, it was decided to conduct around fifteen individual interviews. It has been suggested in the existing literature that in a qualitative approach, while collecting data through interviews, it is necessary to conduct between 5 and 25 (Kvale \& Brinkmann, 2009), or at least twelve interviews (Onwuegbuzie \& Collins, 2007). In selecting the interviewees, out of the eight sampling designs suggested, the 'sequential nested sampling design' was applied in this study (Onwuegbuzie \& Collins, 2007). Accordingly, the participants of the second phase of interviews were chosen from the respondents of the initial survey by contacting them individually, through emails or phone calls.

\subsubsection{Strategies of inquiry for collecting data}

The available literature suggests six major types of data collection methods which can be used in an empirical research: questionnaires, interviews, focus groups, tests, observations, and collection of secondary data (Johnson \& Christensen, 2012; Johnson \& Turner, 2003). In a mixed methods approach, research questions typically include both quantitative and qualitative elements and require both quantitative and qualitative data to be collected and analysed (Onwuegbuzie \& Leech, 2006; Tashakkori \& Creswell, 2007a). Further, it has been suggested that quantitative questions should be specific and may seek quantifiable descriptive responses, or comparable responses to establish relationships of different variables (Onwuegbuzie \& 
Leech, 2006). Similarly, a qualitative question should be as open-ended as possible and generally aim at obtaining insights into a particular context at in-depth level. Qualitative data would be generally obtained in the form of texts or images collected through interviews, observations, or audio-visual resources (Creswell \& Plano Clark, 2007). Among the quantitative approaches, although experimental and non-experimental designs are available (Muijs, 2011), non-experimental designs such as survey, observations, documents, are the most popular options carried out in social sciences (Creswell, 2009; Muijs, 2011). Considering these guidelines, the survey research design was chosen for the quantitative phase of this study, because a survey questionnaire is said to be an instrument meant for collecting measurable data such as opinions, attitudes, and perceptions of the participants (Johnson \& Christensen, 2012).

As for the qualitative strategies of inquiry, it involves different methods such as interviews, observations and documents, and the collected descriptive open-ended data provide more insights and help in better understanding the research themes (Creswell, 2009; Plano Clark \& Creswell, 2008). Among these options, interviews facilitate a researcher to probe intensively or clarify information from the interviewees (Johnson \& Christensen, 2012). Thus, as the interviews are helpful in providing wide and in-depth information about the perceptions, past and present experiences of the respondents, in the latter qualitative phase in this study, the data was collected through individual interviews.

To sum up, by using a sequential mixed methods approach in this study, quantifiable descriptive data were collected initially through a survey questionnaire in the quantitative phase. Subsequently, individual interviews were conducted to generate qualitative data to obtain in-depth understanding of the research themes. The quantitative data obtained and analysed at the primary stage of this study informed the later qualitative phase of data collection.

\section{Research instruments of this study}

For the purpose of collecting quantitative data a modified version of the survey questionnaire from the 2007 national survey on the experiences of international students in New Zealand was used for several reasons. Firstly, the themes of the national survey and this study are similar, such as self-reported academic experiences including the perceptions on the use and 
effectiveness of support services in their institutions. Secondly, the questionnaire administered in the national survey 2007 was reviewed, modified, and tested version of the questionnaire of the national survey 2003, aimed at enhancing the validity of the questionnaire and clarity to the participants. The questionnaire of 2003 was originally designed by considering the literature on international students' experiences, consulting the educational and administrative stakeholders in New Zealand, and conducting several pilot studies with multilingual international students, ultimately assuring the validity and reliability of the survey questionnaire. Thirdly, the 2007 questionnaire was evaluated through an online pilot study which yielded positive results. Moreover, it is suggested that any existing validated instrument can be used, after making necessary modifications (Rudestam \& Newton, 2007). Thus, considering the similarity in themes and reliability and validity of the research instrument, the questionnaire of National Survey of 2007 was administered in this study after having made some modifications.

The researcher was granted permission by the International Division, Ministry of Education, New Zealand, to administer the questionnaire in this study with necessary modifications suitable to the study. With regard to the modifications made in the questionnaire, along with the closed-ended questions a few open-ended questions were added in the questionnaire in order to extract more information from the survey respondents. Johnson and Turner (2003) have suggested that the use of closed-ended and open-ended questions in a single questionnaire allows a researcher to obtain reasonable quantity of information from the participants. Besides this, the national survey questionnaires were designed to cover all international students in multiple sectors such as schools and tertiary level institutions in New Zealand. Therefore, prior to administering it in this study, the questionnaire of 2007 national survey was further modified to suit the requirements of university students only, and to gather data on students from India.

Thus, the questionnaire was re-drafted by incorporating new questions. There included Q.6, on living away from family, Q. 12 field of study, and additional questions related to English language Q. 21, 22, and replacing a single question on self-rated general English language ability with two, distinguishing between academic and everyday contexts Q. 23 and 24. In addition Q. 35 and 36 were developed for postgraduate thesis students, and to Q. 29, 30, 31 as open questions enabling additional information. A new question (Q.47) with 23 items was 
developed to gather information on leisure activities including use of social media, and Q.48 on changes to use of leisure time. Q.50 and 51 were created to gather information on religion and religious observance. A copy of the administered questionnaire can be viewed in Appendix 1. Overall, the questionnaire was reorganised into five sections with sixty questions: Section A: Background information, Sec. B: Educational experiences, Sec. C: Experiences with services and facilities, Sec. D: Social life experiences, Sec. E: Cultural experiences. Further, the revised questionnaire prior to administering it, was tested through four pilot studies (with a mixture of undergraduate and postgraduate international students), to ensure clarity in understanding questions. After considering the views of the participants in the pilot study, a final version of questionnaire was prepared. Finally, the modified survey questionnaire was made available online using the Qualtrics research suite, a software available through university resources, for the convenience of participants intending to complete the survey in an online mode.

For the second phase of the study, a good number of semi-structured interview questions were planned on similar themes of the survey questionnaire. Most of the questions are similar to survey questionnaire with an addition of a few probing questions. The similarity of questions both in survey and interviews were because the survey questions were meant to obtain precise and accurate information, while interview questions were intended to extract contextual and richer data at in-depth level beyond the basic responses of yes or no in the survey. It has been suggested in the extant literature that semi-structured interviews allow the respondents to expand their views whenever it is required, and sometimes may even help them to explain matters that are related to the research objectives (Gray, 2004; Kumar, 2005). Interviews with the participants were expected to yield a great deal of information by questioning directly.

Altogether, the survey questionnaire was intended to collect a moderate quantity of data to arrive at broader assumptions on the experiences and perspectives of the participants, and the following individual interviews were planned to extract extensive and in-depth information on different themes of this study. After preparing the research instruments for both the quantitative and qualitative phases, necessary approval was taken from the university 'Human Ethics Committee' to administer them in this study (Appendix 16). The data was collected in two stages separately, initially by using a survey questionnaire (Appendix 1) and in the later stage with the use of 'semi-structured interview questions' (Appendix 2). 


\subsection{Research implementation process}

Carrying out research involves different steps such as collecting, analysing, validating and interpreting the data (Creswell, 2009; Johnson \& Christensen, 2012). In this study, collection and analysis of both the quantitative and qualitative data were conducted separately, and they were mixed at the interpretation stage. At the point of interpretation of results, data sets from both the approaches were combined and discussed to draw meaningful conclusions from the findings.

\subsubsection{Data collection - Quantitative phase of study}

A number of strategies were applied in the data collection process of this study. It was intended to collect data from 'Indian' international students as they were defined as a specific ethnic group by the government of New Zealand. But I kept in mind the diversity within this particular cohort of students. The survey therefore tried to include both male and female Indian international students in different age groups, studying either undergraduate or postgraduate courses, belonging to different areas in India, speaking different languages and practicing different religions. It was anticipated that besides their adjustment experiences in New Zealand, the inclusion of all these categories may give scope to explore specific features if any that arise because of their diversified status within India.

Then, the researcher visited all eight New Zealand universities to contact eligible participants for this research study. Prior to personal visits the researcher approached student associations and Indian student associations (where they existed) in all the universities for their help in identifying eligible participants. Other than this, wide publicity was given inviting participants to the study through placing special posters at different university locations after obtaining the necessary permissions. Eligible students were advised to contact the researcher either through email or by phone. Additionally, copies of the posters were posted on 'Facebook', inviting eligible students to contact the researcher for their participation in this study. These posters mainly contained information specifying the significance of the study and appealing Indian international students to take part in the survey. 
Besides this, email requests specifying the purpose of the study were sent to friends of the researcher, international offices, different schools and faculties of all the universities with a request to forward the email to Indian international students if they knew any, or if there was anyone listed on their school or faculty lists. Additionally, a request note was published in university student magazines such as 'Salient' of Victoria University of Wellington, and in Indian community newspaper published from Auckland (Indian Newslink). After attracting a few students' attention, a 'snowball approach' was applied to contact their friends who might participate in the survey.

When interested and eligible students made contact, a participant information sheet was emailed to them seeking their consent to take part in the survey in whichever mode they preferred - either through online or by using a hard copy (Appendix 13). The information sheet contained the details of the researcher, the purpose of the study, assuring anonymity and confidentiality of the information. Consequently, on receiving their informed consent, a link set for the online survey questionnaire was sent to them along with a request to complete the survey within a week's time. All the participants opted for online mode. Reminder emails were sent to the participants twice, for completing the survey at an early date.

Additionally, to increase the response rate, for a limited period of time, a special incentive was offered to the participants to encourage them to complete the survey within a specified date. Offer of small incentives was indicated in the Ethics application and no objections were received regarding this matter from the said committee. The incentive offered was a donation of \$5-00 in their name or any preferred name to the 'Cancer Society of New Zealand' for those who complete the online survey and to inform the researcher within a stipulated timeframe. Around $20 \%$ of the respondents to the survey were encouraged by this incentive. For those who informed, the specified amount was paid to the Cancer Society of New Zealand in Wellington and the copies of receipts were emailed to the students.

With all these efforts, the online survey link was sent to a total of 181 eligible students, and 109 completed surveys were received with a response rate of above $60 \%$. The number of completed surveys exceeded the pre-set target of at least 100 completed surveys for this study. 


\subsubsection{Data collection - Qualitative phase of study}

Among the respondents of the initial survey, a few showed greater levels of interest in this study, and voluntarily helped the researcher even in identifying and encouraging other eligible participants to take part in this study. Based on the criteria as explained above in the quantitative phase of data collection, the participants for individual interviews were selected by using a convenience sampling approach. The criteria considered for selecting the participants were gender, age, level of study, religious backgrounds, and university affiliation. They were asked to share their language, academic and social life experiences on themes with which they were already familiar through the earlier survey. The interview questions were designed with open-ended questions to explore detailed and in-depth information from the participants. From the willing participants of the interviews too an informed consent (Appendix 15) was taken expressing their approval to take part in the study.

For the interview, each participant was allotted around an hour, and the interviews were recorded digitally with the permission of the interviewees. The interviews were conducted in different modes of face to face, Skype, and phone according to their convenience. Each interview was started with casual conversations prior to start the actual interviewing, to establish a comfortable setting for the interview process. While conducting interviews, the 'Interview guided approach' was followed (Johnson \& Turner, 2003, p. 305). This approach suggests that the main questions related to the themes may be used in a flexible manner by either rewording the questions or by changing the sequential order according to the situation. Other than this, special care was taken to note the non-verbal communicative aspects, such as facial expressions, voice modulations, emotions, or hand gestures (Onwuegbuzie, Leech, \& Collins, 2010), to improve the understanding of the data. The completed recordings were uploaded and preserved as computer audio files. Later, individual interviews were transcribed verbatim for analytical purposes. In appreciation of their contribution to the study each interviewee was offered an incentive of a \$10-00 'international calling card'. For those participants of the interviews other than face to face mode, calling cards were sent to their mailing addresses. 


\subsubsection{Demographic profiles of the participants}

\section{Of the survey questionnaire}

Out of the total of 109 respondents to the online questionnaire, 104 respondents opted to address questions related to the age and gender. Of them, $97 \%$ were under the age of 35, and of them $76 \%$ were under 30 years and just over a third (34\%) were under 25 years of age. Three quarters were male $(\mathrm{N}=78)$. Similarly, $82 \%$ were postgraduate students, with $21 \%$ enrolled for a master's degree, $36 \%$ for doctorates and others for postgraduate diplomas or similar. Only $18 \%$ were engaged in Bachelor degree studies. The participants were engaged in a range of studies such as Business and Management (22\%), Medicine/Health Sciences (17\%), Engineering (10\%), Computing and IT courses (9\%), Agriculture/Horticulture (6\%), Humanities and Social Sciences (6\%), and in other courses (30\%). Among the courses specified in the other courses were mostly science courses with specialisations such as Environmental sciences, Marine biology, Veterinary science, and Nanotechnology.

The participants were studying at all the eight universities in New Zealand. The percentages of participation are: University of Otago (22\%), Victoria University of Wellington (21\%), AUT (21\%), Massey University (20\%), University of Waikato (7\%), University of Auckland (4\%), University of Canterbury (2\%), and Lincoln University (2\%). No specific reasons were identified for less participation from the Universities of Auckland and Waikato. However, the city of Christchurch where University of Canterbury and Lincoln University were located was severely affected by an earthquake during the timeframe of data collection for this study.

For most of the respondents $(89 \%)(\mathrm{N}=105)$ studying in New Zealand was their first experience of living outside India, and a majority (67\%) were living away from their families for the first time. Similarly, $84 \%$ of the respondents reported that they were staying for less than three years in New Zealand, and the others (16\%) stated that they were in New Zealand for more than three years.

The participants came from different states in India and they belonged to the states of Assam, Andhra Pradesh, Karnataka, Kerala, Tamilnadu, Delhi, Gujarat, Maharashtra, Punjab, Haryana, 
Chandigarh, Bihar, Goa, Uttar Pradesh, Uttarakhand, Jharkhand, Orissa, Rajasthan, Puducherry and West Bengal.

With regard to the religion, $76 \%$ of the participants belonged to Hindu religion, $12 \%$ were Christians, 5\% Sikhs, 2\% Muslims, 1\% Buddhist, 3\% were students with no religion, and 2\% of the participants did not wish to answer this question.

\section{Of the individual interviews}

Out of the total of fifteen participants in the interviews, eight participants were in the age group of 17-24, and the remaining seven participants in the 25-29 age group. Ten of them were male and the other five female. Three of the interviewees were undergraduate students, six of them were engaged in their postgraduate studies other than doctoral studies, and the other six were involved in their doctoral studies. The participants were engaged in a range of studies such as physiotherapy, environmental science, agriculture, accounting, film studies, human resources management, physics, humanities, health sciences, civil engineering, marine biology, education, computer science and Law. The interviewees participated from six universities in New Zealand. Eight participants from Victoria University of Wellington, two from University of Otago, two from University of Waikato, one each from University of Auckland, Massey University, and Auckland University of Technology. The interviews were held face to face with eight participants, through 'Skype' with six participants and over phone with one participant. Similar to survey respondents, the interviewees represented different religions and states in India.

Although, the respondents of the survey and the participants of the interviews belonged to different states of India with different cultural backgrounds, and affiliated to different religions backgrounds, they did not mention any issues related to their individual differences in background which helped or hindered their experiences of adjustment to external challenges in New Zealand. On the other hand, it also became apparent that in a foreign situation they all felt to be 'Indian' because they were treated as such by others. The individual details of the interviewees are provided in the following Table 3.1. Pseudonyms were used for the participants of the interviews to protect their identity. 
Table 3.1-Details of the interviewees

\begin{tabular}{|c|c|c|c|c|c|c|}
\hline $\begin{array}{l}\text { Sl. } \\
\text { No. }\end{array}$ & Pseudonym & $\begin{array}{l}\text { Age } \\
\text { group }\end{array}$ & Gender & Course & Field of study & University \\
\hline 1 & Kishore & $25-29$ & Male & Postgraduate & Physiotherapy & $\begin{array}{c}\text { University of } \\
\text { Otago }\end{array}$ \\
\hline 2 & Vineel & $17-24$ & Male & Postgraduate & $\begin{array}{c}\text { Environmental } \\
\text { Science }\end{array}$ & $\begin{array}{c}\text { University of } \\
\text { Auckland }\end{array}$ \\
\hline 3 & Satya & $25-29$ & Female & Postgraduate & Agriculture & $\begin{array}{c}\text { Massey } \\
\text { University }\end{array}$ \\
\hline 4 & Pranith & $17-24$ & Male & Postgraduate & Accounting & $\begin{array}{c}\text { Victoria } \\
\text { University of } \\
\text { Wellington }\end{array}$ \\
\hline 5 & Monish & $17-24$ & Male & Postgraduate & $\begin{array}{c}\text { Human } \\
\text { Resource } \\
\text { Management }\end{array}$ & $\begin{array}{c}\text { University of } \\
\text { Waikato }\end{array}$ \\
\hline 6 & Rajasekhar & $25-29$ & Male & Postgraduate & Physics & $\begin{array}{c}\text { Victoria } \\
\text { University of } \\
\text { Wellington }\end{array}$ \\
\hline 7 & Rashmitha & $25-29$ & Female & Postgraduate & $\begin{array}{c}\text { Health } \\
\text { Sciences }\end{array}$ & $\begin{array}{c}\text { University of } \\
\text { Otago }\end{array}$ \\
\hline 8 & Razia & $17-24$ & Female & Postgraduate & Education & $\begin{array}{c}\text { Victoria } \\
\text { University of } \\
\text { Wellington }\end{array}$ \\
\hline 9 & Jasmine & $17-24$ & Female & Postgraduate & $\begin{array}{c}\text { Human } \\
\text { Resources } \\
\text { Management }\end{array}$ & $\begin{array}{c}\text { University of } \\
\text { Waikato }\end{array}$ \\
\hline 10 & Sunil & $17-24$ & Male & Postgraduate & $\begin{array}{c}\text { Civil } \\
\text { Engineering }\end{array}$ & $\begin{array}{c}\text { Auckland } \\
\text { University of } \\
\text { Technology }\end{array}$ \\
\hline 11 & Suresh & $25-29$ & Male & Postgraduate & $\begin{array}{l}\text { Computer } \\
\text { Science }\end{array}$ & $\begin{array}{c}\text { Victoria } \\
\text { University of } \\
\text { Wellington }\end{array}$ \\
\hline 12 & Prakash & $25-29$ & Male & Postgraduate & Law & $\begin{array}{c}\text { Victoria } \\
\text { University of } \\
\text { Wellington }\end{array}$ \\
\hline 13 & Dinakar & $25-29$ & Male & Undergraduate & Humanities & $\begin{array}{c}\text { Victoria } \\
\text { University of } \\
\text { Wellington }\end{array}$ \\
\hline 14 & Victor & $17-24$ & Male & Undergraduate & $\begin{array}{l}\text { Faculty of } \\
\text { Science }\end{array}$ & $\begin{array}{c}\text { Victoria } \\
\text { University of } \\
\text { Wellington }\end{array}$ \\
\hline
\end{tabular}




\begin{tabular}{|c|c|c|c|c|c|c|}
\hline 15 & Mahima & $17-24$ & Female & Undergraduate & $\begin{array}{c}\text { Faculty of } \\
\text { Humanities } \\
\text { and Social } \\
\text { Sciences }\end{array}$ & $\begin{array}{c}\text { Victoria } \\
\text { University of } \\
\text { Wellington }\end{array}$ \\
\hline
\end{tabular}

\subsubsection{Data Analysis procedures}

In sequential designs, usually the researcher follows data analysis procedures separately for the data collected through quantitative and qualitative approaches (Creswell, 2009; Plano Clark \& Creswell, 2008). The standard approaches for quantitative and qualitative data are descriptive and inferential analysis, and coding and thematic analysis respectively. As indicated in this study both the quantitative and qualitative data were analysed independently using the standard approaches as detailed below.

\section{Quantitative data analysis}

Most of the quantitative data in this study were collected with numeric values with a few exceptions of textual responses for some of the open-ended questions in the survey questionnaire. To obtain sense of the numerical data, they were analysed statistically. Two kinds of procedures for the quantitative data analysis have been suggested in the literature, such as descriptive and inferential statistical procedures (Botti \& Endacott, 2005; Johnson \& Christensen, 2012). While the descriptive statistical analysis presents the data with frequency distributions and graphical representations, to extract a particular sense out of the data in the form of quantities and percentages, the inferential statistics may deduce possible meanings from the data on the characteristics of the study population through measures such as mean or median. In this study, the descriptive statistical procedures were used to analyse the quantitative data. The intention of the quantitative phase was to identify the trends and to formulate broader categories of views of the sample population on different variables, and to put a focus on these variables while investigating further in the next phase of study.

For this purpose, a software called Statistical Package for Social Sciences (SPSS), provided by Qualtrics research suite (available in the university), was used to present the data in the form 
of cross-tabulations (for showing different variables in rows and columns), and different kinds of charts such as pie or bar charts (to show the frequency distributions). By using these frequency distributions related to different variables (e.g., age or gender) through graphs and tables along with quantities and percentages, attempts were made to draw meaningful conclusions on different variables. With regard to the responses of the open-ended questions in the questionnaire of the quantitative phase, analysis procedures applied to the text responses obtained in the qualitative data were applied. The procedure will be explained below.

\section{Qualitative data analysis}

Qualitative data analysis procedures are relatively new when compared to quantitative data analysis procedures, and it appears from the extant literature that many qualitative researchers are looking for more robust procedures (Johnson \& Christensen, 2012). Previously, a framework was conceptualised for mixed methods data analysis based on two underlying principles, such as 'representation and legitimation' (Onwuegbuzie \& Teddlie, 2003, p. 353). Representation suggests extracting reliable information from the generated data, whereas legitimation refers to interpreting the data in a valid manner. Grounded in these principles the framework proposed a seven step model for analysing the data in mixed methods approach which includes both the components of quantitative and qualitative data. The different steps involved in this model are: data reduction, data display, data transformation, data correlation, data consolidation, data comparison, and data integration. However, the last two steps were said to be important for mixed methods researchers (Johnson \& Christensen, 2012). Explaining the process in a simplified manner, Johnson and Christensen (2012) indicated a few basic measures such as transcribing the data from the audio recordings, segmenting the text into meaningful chunks, and coding the data by labelling different categories (the process of segmenting and coding will be observed simultaneously), probing for identifying relationships between the themes, and interpreting the data to draw conclusions from the study.

Following the guidelines, the qualitative data obtained in the first phase was analysed initially while analysing the quantitative data. After completing the individual interviews in the second phase, the obtained data was analysed. The process began initially by transcribing the data. Then, each individual interview transcription was divided into chunks according to different themes of the study, such as language and academic experiences. In the process, responses of 
all the interviewees were organised into different categories thematically, and later the responses of all the interviewees for each theme were pooled up together. As a result, theme wise responses of all the interviewees were available for critical observation. The theme wise responses were further segmented into different sub-categories and coded (e.g., language issues, coping strategies). Finally, reading of the data several times helped the researcher to identify the specific trends and opinions of the participants, to make comparisons between the variables, and to identify the patterns and relationships to make interpretations of the data.

Thus, both the quantitative and qualitative data were analysed separately and the results were integrated at the interpretation stage. The descriptive statistics of the quantitative data in the form of frequency distributions represented in graphs and tables, and the extracted patterns and relationships of the qualitative data established through the thematic analysis process, together resulted in summarised data for the sample population. Consequently, this summarised data were interpreted to understand the research issues, and to draw conclusions for this study. To maintain the reliability of the data and validity of the procedures, certain validation/legitimation measures were taken by the researcher which will be described below.

\subsubsection{Data validity/ legitimation}

It is strongly suggested that a researcher should assess both the quantitative and qualitative data for its validation/legitimation (Johnson \& Christensen, 2012; Leech \& Onwuegbuzie, 2010; Onwuegbuzie \& Johnson, 2006; Tashakkori \& Teddlie, 2003, 2008). The assessment involves examination of internal and external threats to the validity of quantitative data, and any threats to trustworthiness, credibility, dependability, authenticity, verification, plausibility, applicability, confirmability, and/or transferability of the qualitative data (Leech \& Onwuegbuzie, 2010, p. 65). Considering the combination of dual strengths and limiting weaknesses from both the quantitative and qualitative approaches in a mixed methods approach, Onwuegbuzie and Johnson (2006) suggested use of the term 'legitimation' instead of 'validity', while referring to the validity issues of mixed methods approach. Accordingly, in this study necessary measures were taken to address possible threats to the validity concerns of the study. 
To check the internal validity of the components of the survey questionnaire, the questions were thoroughly examined to determine whether or not they elicited the current conditions through the responses of the respondents. Other than this, both the supervisors reviewed the components of the questionnaire to assess the capability of the instrument in extracting appropriate and measurable data. Besides these, as the questionnaire was modified, incorporating a few new questions specific to a particular group of students such as thesis students, pilot studies were undertaken to evaluate the consistency of the instrument. As for confirmation of the external validity, it may be noted that originally the questionnaire was systematically designed and extensively used in several national surveys and other similar studies in New Zealand to examine the experiences of international students. The responses of the pilot study respondents also helped to determine the reliability of the instrument.

To ensure trustworthiness, credibility, dependability, transferability and other similar concerns about qualitative data, different strategies were adopted in this study. Special attention was paid to following the guidelines and procedures in the process of data collection and data analysis. The set procedures were carried out as suggested in the sequential order. The researcher became familiar with the different procedures associated with each phase of the study. The researcher personally transcribed all the interviews to avoid errors and to ensure trustworthiness of the data. A few times the researcher received instructions from the supervisors to verify whether the audio recordings corroborated the words transcribed. While making comparisons or establishing the relationships, the data were thoroughly checked for threats such as credibility and authenticity to make accurate interpretations. The clarity in observing the procedures in a systematic way enhanced the quality of data which enabled the researcher to draw trusted inferences from the collected data. Other than this, the whole data collection and analysis processes were monitored by the two supervisors continually. Thus, the intervention of the supervisors helped the researcher to avoid any procedural errors and to draw fair conclusions from the findings of the study. To extract appropriate data, as well as to maintain the stipulated ethical standards, the participants of each phase of study were well informed about the purpose and context of the study prior to their participation in the study. Thus, a good number of precautionary measures were taken to lessen the possible threats to the validity as well as to increase the quality of the study. 


\subsubsection{Data interpretation}

Tashakkori and Teddlie (2003, p. 37) established the criteria for evaluating findings in a mixed methods research. The criteria indicate measures for checking consistency of inferences within each approach (quantitative and qualitative) and also with each other within a study. Similarly, it was suggested that the researcher check the consistency of interpretations with other studies which had similar results. Other than these, the researcher needs to check for the trustworthiness of interpretations by excluding the other possible dishonest interpretations using the same findings. Additionally, while introducing the integrative framework for evaluating the quality of mixed methods, Tashakkori and Teddlie (2008) added another measure of integrative efficacy which denotes whether meta-inference of quantitative and qualitative data integrated effectively or not. Other than this, Onwuegbuzie and Johnson (2006), indicated the scope for misinterpretation of the data when a researcher compromised, particularly in case where the participants of the study and the researcher belong to same ethnicity. As a remedy to prevent this threat it was suggested that the data and the interpretations should be peer reviewed by other people who are familiar with the procedures. All these measures suggest that a researcher should be aware of these threats while interpreting the data of a study, and expect precautionary measures to be taken in enhancing the quality of the study.

In this study, the data were analysed independently at each phase of the quantitative and qualitative study, and finally both the inferences were converged at the point of interpretation. The inferences of earlier quantitative study were also utilised for identifying exact areas to be focussed in the second phase of the study. For instance, the stimulating aspects observed in the initial quantitative study in terms of English language or academic experiences of the participants, were further probed in detail during the individual interviews for attaining detailed clarifications and information. While interpreting the meta-inferences, both the data were combined and compared in a coherent manner to establish relationships between the views of the participants of each study, and to elucidate meaningful conclusions on the study themes. While making interpretations, special attention was paid to extract accurate meanings with apt interpretive distinctiveness. Similarly, while integrating the results of each phase of study to frame meta-inferences, adequate measures were taken to avoid misrepresentation of the data. 
As it happened in the case of generating findings from the data, the two supervisors closely monitored the process of interpretations and examined the elucidations made over the findings. Overall, to avoid any inconsistency in interpreting the data of quantitative and qualitative phases or while making meta-inferences, necessary care and precautionary steps were followed by the researcher.

\subsection{Ethical considerations}

The university regulations require ethical approval from the Human Ethics Committee whenever a research involves human participants. Application of ethical principles in a study, makes both the researcher and the participants aware of the values such as 'relevance, responsibility, respect and reciprocity' (De Wet, 2010, p. 312). Importantly, it has been indicated that ethical issues require due consideration in all the procedures of the study such as data collection, analysis, and reporting (Creswell, 2009), including the referencing of authors genuinely while using their contributions in the study (Johnson \& Christensen, 2012).

Accordingly, necessary approvals were obtained from the Human Ethics Committee of the Victoria University of Wellington as this research involved data collection using student participants (Appendix 16). While collecting the quantitative data initially, the potential participants were invited to take part in the study on voluntary basis. For those willing students a participant information sheet (Appendix 13) was sent to them requesting their informed consent. The contents of the information sheet briefed the objectives of the study, amount of time required, assurance of anonymity, and confidentiality of the data they provided. After obtaining their acceptance only, an online link was set up for the survey was sent to them. The researcher considered the participants unwilling if they did not respond with their consent. Even for those who expressed their interest to take part in the survey and received the survey link, except for sending reminding emails twice in a general manner, the researcher did not pressurise them to submit the completed survey.

Similarly, the participants of individual interviews were also given detailed information through an information sheet (Appendix 14) on the research issues, required time and purpose of the interviews, and only after obtaining their written consent, individual interviews were held according to their suitable time and mode. Participants were assured of liberty to withdraw 
from the study at any time before the data analysis process. Further, they were assured of the confidentiality of the information they would reveal. Further, to protect their privacy the names of the institutions of their study or places of living were kept anonymous. Specific guidelines were followed even in reporting this study by honestly referencing the contributions of different authors in a stipulated manner. The continuous monitoring of the supervisors of the study also helped the researcher to maintain the ethical standards according to the specified guidelines.

\subsection{My role as a researcher}

My personal experiences as an immigrant to New Zealand from India set the stage for my research. These experiences resulted in several adjustment challenges, being new to place, people and environment, which were beyond my expectations initially. Consequently, these challenging concerns shaped my interest in studying the experiences of Indian international students in New Zealand. The review of literature on international students further deepened my feelings as the literature mostly disclosed the adverse experiences of international students both in their academic and social lives being distant from their families and home countries. The significant issue I have noticed was despite experiencing challenges, international student numbers are increasing worldwide yearly. The other aspect I have noticed is that although the educational institutions are providing facilities and services to these particular group of students, those services are not being utilised as intended. These observations drew my attention to this study with a strong commitment to explore specific reasons for this situation by examining the experiences of current international students. It is expected that the outcome of this study may offer solutions at least to a few of these problems.

These personal experiences and expectations led me to a greater level of commitment which helped me to undertake the study with utmost care. Thus, because of this reflexivity, I was conscious that my personal experiences should not bring biases to the research process during the stages of data collection, analysis and interpretation. Further, I was cautioned that when a researcher belonged to the social or cultural group of participants of the study there might be a scope for misinterpretation of the data (Onwuegbuzie \& Johnson, 2006; Onwuegbuzie et al., 2010). I am also aware that the positionality of a researcher in social sciences research has been much debated in the past two decades (Moser, 2008). Moreover, these different aspects of 
positionality such as race, class, gender mainly affect the maintenance of required ethical practices of a study (Hopkins, 2007). Bearing these principles in mind, I set aside myself and my personal feelings, as far as practicable, during different phases of this study, to achieve reliability and credibility of the findings of this study.

Further, to avoid any inconsistencies of interpretation, a peer debriefing process has been suggested and this means the interviewer being interviewed initially by a non-participating person (Tashakkori \& Teddlie, 2008). I have accomplished this task which helped me in evaluating the role of hunches, which might distract the researcher from proper understanding and representation of the research issues accurately. Moreover, since the beginning of the research all important steps involved in the process were closely monitored by the two supervisors of the study which helped the researcher in accessing continuous guidance and suggestions to deal with the issues in a fair manner. Further, the use of technology for collecting the data online and analysis procedures also helped to avoid negative impacts arising from the positionality of the researcher. In total, all the activities of data collection, analysis, and interpretation procedures were structured and performed in a way to maintain reliability and validity of the study.

\subsection{Summary}

In this chapter the research approach applied in this study was detailed. Firstly, the framework related to the designing of the research, and the criteria and procedures observed in selecting an appropriate research approach for this study were described. These matters are broadly related to different types of research approaches such as quantitative, qualitative and mixed methods approaches that are available in Social Sciences. Secondly, matters related to research planning and implementation procedures were described. These procedures are mainly associated with the sampling issues, strategies of inquiry, data collection, analysis, and interpretation. This section also includes a note on the demographic profiles of the participants of the study. Finally, a brief account on the Ethical considerations and the role of the researcher were discussed. The following chapter will present the details of the findings of this study. 


\section{Chapter 4}

\section{Research findings: Choice of New Zealand for studies and English language proficiency}

\subsection{Overview}

The findings from the survey and the interviews have been organised into three chapters. These findings shed light on five themes which are: factors involved in choosing to study in New Zealand, English language proficiency, academic experiences in New Zealand, their perceptions on the use of support services in their universities, and social life in New Zealand.

The current chapter is organised into sections presenting the findings on the first two themes: factors which influenced Indian students to choose New Zealand as their study destination, and the English language proficiency of the students. As described in Chapter 3, the data reported here were generated in two stages, an online survey and later from in-depth interviews. The online survey was designed to generate a broad overview of student perspectives and experiences, and the in-depth individual interviews to obtain richer and more nuanced insights into the experience of Indian international students. Mostly, the results from the analysis of the survey questionnaire will be presented in the form of graphs and tables, and the analysis of the interview data will be presented as narrative text supported by quotes from the participants. Pseudonyms have been used for the interviewees to protect their anonymity.

In each section, the views, experiences, and challenges of the participants related to a specific theme will be provided. Along with these, a note on the strategies applied by Indian international students to overcome their challenges, and some suggestions given by them for improvement of conditions will be presented. Finally, each chapter will end with a brief summary on the key findings of the chapter. 


\subsection{Motivating factors for choosing New Zealand}

In response to the survey question as to whether New Zealand was their first choice of study destination, $55 \%$ of the total 105 respondents who answered this question responded positively, and $45 \%$ negatively. When they were asked to indicate other choices of study destinations if any, a popular choice appeared to be the United States of America (45\%), followed by Australia (26\%), the United Kingdom (19\%) and Canada with 4\%. It is notable that the majority of the respondents' listed English speaking countries, while only a small section (6\%) favoured other destinations such as Germany.

\subsubsection{Common factors in choosing place of study}

A total of 178 responses $(\mathrm{N}=97)$ were provided to an open-ended question in the survey that asked respondents to specify reasons for choosing New Zealand as a place of study. A majority of respondents (54\%) offered multiple reasons. While broad categories of key factors identified by the participants are shown in table 4.1 below, a full list can be viewed in Appendix 3 .

Table 4.1 - Key factors influencing Indian students to choose New Zealand for study

\begin{tabular}{|l|c|c|}
\hline Reasons for choosing New Zealand & $\begin{array}{c}\text { Frequency of } \\
\text { responses }\end{array}$ & $\begin{array}{c}\text { \% } \\
\text { (N=178) }\end{array}$ \\
\hline $\begin{array}{l}\text { Good courses/quality education/ } \\
\text { university ranking/good faculty/ } \\
\text { interesting project or subject }\end{array}$ & 51 & 29 \\
\hline $\begin{array}{l}\text { The image of New Zealand in terms of } \\
\text { peaceful and safe country/friendly } \\
\text { people/relaxed lifestyles of the people }\end{array}$ & 50 & 28 \\
\hline $\begin{array}{l}\text { Low fees } \\
\text { Scholarships }\end{array}$ & 23 & 08 \\
\hline Career prospects & 13 & 07 \\
\hline Personal and other factors & 26 & 13 \\
\hline
\end{tabular}


The responses include broadly four categories of reasons for choosing New Zealand as a study destination by the Indian international students. They are: academic considerations such as quality of education, good faculty, and interesting topic; the image of New Zealand which includes the perception of safe environment, and friendly people; financial considerations such as award of scholarships or low fee policy of the New Zealand government; career prospects; and personal factors such as self-interest, friends' influence, family ties and notions about easy admission and immigration procedures. Racism in other countries was also indicated by the respondents as a reason for studying in New Zealand. Out of the total responses, remarkably only one participant indicated that the English speaking environment was one of the reasons along with other factors to study in New Zealand.

The interviews also provided an opportunity to explore the reasons why these students chose to study in New Zealand. The factors that were mentioned by the interviewees were: reputation of the institution; reputation of the faculty in the chosen field of study; good value for money; opportunities after the completion of study; the dream of studying overseas; opportunity to work part-time while studying; similarity to British education system; English speaking environment; encouragement given to the field of research; availability of desired courses; and the political stability of New Zealand. One of the interviewee explained that the university where he did his master's degree was one of the renowned universities in India in terms of academic and research achievements, and there existed a trend among the masters' students to go overseas for doing $\mathrm{PhD}$. The interplay of multiple factors came through in the interviews. Some of these factors are explained below.

One undergraduate male student, Victor stated that his parents' intention to send him out of the home country and at the same time heavy expenditure involved in undertaking studies in the US made him select New Zealand as his study destination. He described in detail how he analysed several factors during his decision making process to undertake studies in New Zealand:

My parents and I didn't want to study back at my home country, and wanted to get outside and explore. I started looking at schools all over the world. I actually even 
wrote my SAT $T^{2}$ looking at American schools and there was a time when the recession hit, and they stopped giving scholarships for international students. Then I decided not to study in the US as it is so expensive to study there (without a scholarship). Then I did some travelling and I did some work and then again I decided that I wanted some study. I looked at schools in Australia, New Zealand and Kenya. I crossed out Kenya because I thought that it would be nice to work in Kenya but I don't want to live there right now. Then I thought of either Australia or New Zealand. New Zealand is cheaper compared to Australia and it is better for marine biology. There is no wild life here and with rich forests. I have friends here and I travelled here before. Then I decided that. I would like to live in New Zealand a bit more because the people are nice and it's a nice culture and a friendly place. Last time when I got to (the name of the city), I just fell in love with this city as we don't need even a car and we can walk everywhere.

For Mahima, the perception of political stability and the reputation of the University City as a creative hub convinced her to choose New Zealand for her studies. For many postgraduate students, particularly for the doctoral students, the provision of financial support and research encouragement motivated them to pursue their studies in New Zealand.

Perceptions about security conditions in New Zealand influenced the study choice of some students. Two interviewees (13\%) indicated that safety and peaceful environment were factors that influenced their decision to select New Zealand as a study destination. This percentage is closer to the percentage of survey responses (14\%) for this question. One of the interviewees emphasised safety as the primary reason, and being offered a scholarship as another factor that influenced her decision:

Rashmitha: The first reason was that it is a safe country.... Next other big reason was the scholarship which this university gave me. I didn't want to lose this opportunity. So, I just grabbed it.

\footnotetext{
${ }^{2}$ A standardized admission test taken by prospective students to get admission into the US colleges.
} 
Availability of specific courses was an important factor for some to study in New Zealand. Two participants pursuing their undergraduate studies in film and theatre studies stated that the reputation of New Zealand in the film industry influenced their decision.

Additionally, when the interviewees were asked whether they had any specific reasons for selecting a particular university in New Zealand, they shared fascinating stories. Rajasekhar, who completed his master's degree at a highly regarded Indian university, and whose doctoral thesis was under examination at the time of the interview, remarked that he chose his university because it had a research position available.

This is the only place for which I've applied in New Zealand. There used to be a website called tiptop.org which actually had all the positions in the field of Physics for PhD and postdoctoral research related programmes. In your area of interest you will find research positions in different countries and so this was the only position I had, which was suitable for me in New Zealand and for this I have applied.

\subsubsection{Significance of financial issues and support}

In response to a question in the survey on how financially difficult is it for them/ their families to pay for education in New Zealand, 35\% of the total 105 respondents reported that it was very or extremely difficult, and $40 \%$ reported that it was either slightly or moderately difficult for them or for their families (Figure 4.1below). Thus, financial considerations could also be a valid reason for these students as they perceived New Zealand to be a cheaper option compared to other countries. 


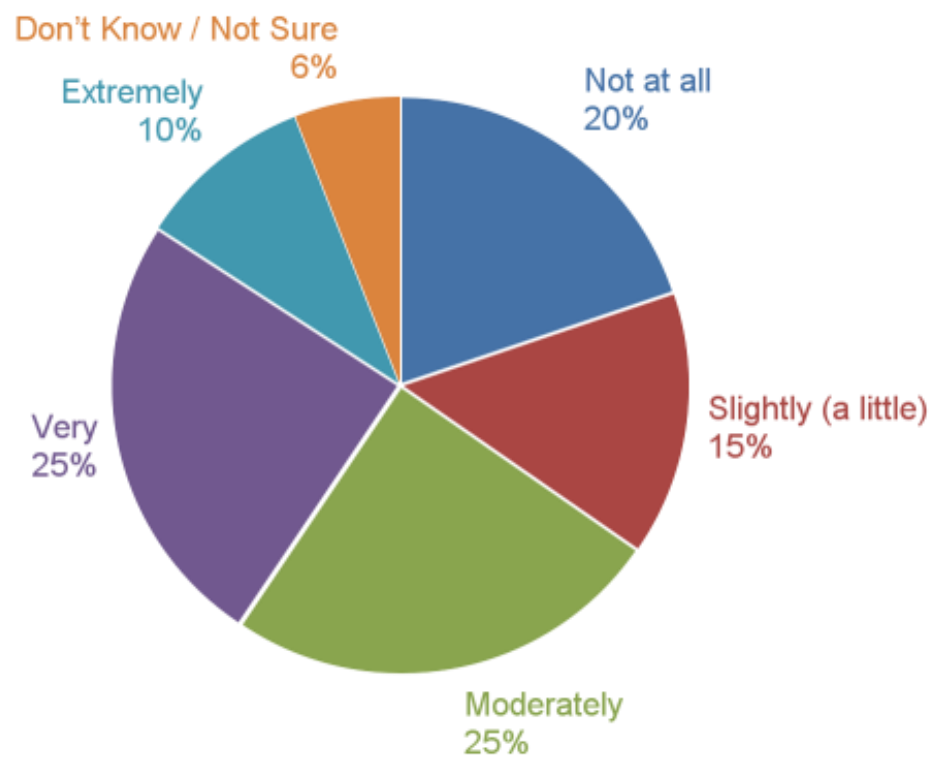

Figure 4.1 - Difficulty in paying for their education in New Zealand

Six of the 15 students interviewed were doctoral students, and all of them indicated that offers of financial support influenced their decision-making as to where to study. A doctoral student Prakash expressed his joy on attaining additional benefit along with the financial support as below:

Prakash: I had no particular jurisdiction or university in my mind where I should undertake my PhD study. The main idea was to get a scholarship from any decent university. I was focusing majorly in the UK, some universities in Canada and Australia. I had heard about New Zealand universities as well offering scholarships. Then I started sending my proposals across to various universities in the UK, Canada, Australia and New Zealand as well. I got accepted in New Zealand. Secondly, my supervisor is well established and globally recognised person in this field.... As I got both scholarship and good supervisor, I chose New Zealand to study [for] my PhD.

Moreover, many of the doctoral students appreciated the efforts of the New Zealand government for its encouragement and financial support towards the research field. To illustrate this one of the doctoral student's comment is provided below: 
Kishore: I was just quite happy at that time to know New Zealand government supports much for research. The scholarship to promote research is a good thing actually. That thought really inspired me and I didn't try anywhere else, when I applied I just got this.

Similarly, charging of domestic fee for doctoral students also influenced some, as it lessens their financial burden.

Satya: Actually when we were doing masters [in India] there were few of my classmates who came over here to New Zealand, since then they were just telling me about New Zealand and the universities over here, and to get an admission in PhD over here is bit easier and they charge the domestic fee and not the international fee.

For another doctoral student lack of financial ability to go to other study destinations and preference for English speaking environment in New Zealand led his decision making process. He illustrated his thoughts as below:

Rajasekhar: I wanted to go to the United States, but my economic background didn't support that, as we have to apply to a number of universities and it costs a lot. I was looking for some alternative opportunities and I applied in Europe and other places. There were three positive offers by the end of my course. One offer from Germany, one from Italy and another from New Zealand. The thing with the Italy offer was really good from a place called Cissa. But it was a conditional offer. I had to go and spend some time there and I had to appear for an exam at the end of 6 months, and if I actually make it through they would grant me a seat and the process would take a while. Then the German one is the same and the professors said that they were happy for me to come over there to do PhD, but he was about to go on leave for 9 months. As I can't wait for such a long time, I've discarded that. Meanwhile I've got an opportunity from New Zealand. I had to choose between Italy and New Zealand. It will take a while with a conditional offer if I go 
to Italy, and it was straight forward with New Zealand. Another big factor was actually I really wanted to improve my English skills. So I chose New Zealand.

The less expenditure towards the study costs in New Zealand persuaded a few students. Two of the masters' students shared their views as below:

Jasmine: I always wanted to pursue my master's from abroad and I selected New Zealand ... because of the financial reasons. It is quite cheap compared to the other countries.

Suresh: One deciding factor is that New Zealand is one of the cheapest places to study when compared to other developed countries like the US, the UK, Australia or Canada.

Consideration of other reasons along with the affordability motivated another postgraduate student to study in New Zealand. He shared his analysis as below:

Pranith: In India we have a dream of studying overseas. So we have categorized 2 or 3 countries such as the US, the UK and Australia. The fourth option was New Zealand. For going to the US, I could not get a better score ... than 700 in GMAT, for UK it has reached saturation point and overseas students/people are not satisfied with the state of facilities, and for Australia it was [identified with] recent racist attacks. In New Zealand it is easy access, better life and less expensive.

Besides the perception that New Zealand was less expensive, provision of financial support in the form of scholarships was an important encouraging factor for some students. As many as 15 respondents to the survey stated that the offer of a scholarship was the only factor that influenced their decision to choose New Zealand as a destination for further studies. The survey revealed that $23 \%$ of the total of 105 participants of the survey were offered scholarships for their study.

Another consideration that comes through this study is the charging of domestic fees for doctoral students in New Zealand. Out of $15 \%$ respondents who classified themselves as 
International $\mathrm{PhD}$ student with domestic fee, six of them stated that they were influenced by the factor of paying domestic fee. Out of these six, for four respondents it was an exclusive factor influencing their decision to study in New Zealand. Altogether 38\% of the respondents of the survey were either fully or partially supported for their studies in New Zealand. In another dimension, among the total respondents $57 \%(\mathrm{~N}=105)$ indicated that they were involved in either master's degree or doctoral studies. The rest of the respondents were involved in either undergraduate $(18 \%)$ or postgraduate certificate or diploma courses $(25 \%)$. Hence, among the master's or doctoral students, $67 \%$ were financially aided either partly or fully for their studies.

\subsubsection{Influence of specific long-term goals}

International students sometimes have long term goals and generally consider career prospects and permanent residency options while making their choice of a study destination (Baas, 2010). In the survey 15 responses indicated reasons related to work and immigration opportunities in New Zealand. These responses indicate that the respondents had considered better career opportunities, work culture, and immigration policies of New Zealand while making their decision to study in New Zealand. This situation implies that they have long-term goals and assessed the conditions for future growth and settlement opportunities in the host country, before making decision on their study destination. To an open ended question in the survey related to this matter, one of the male participant specified as below:

R.84: I found Biotechnology has a good opportunity in New Zealand.

Similarly, another female participant stated that:

R.85: When I reviewed, New Zealand is greatly promoting overseas students to come and study ... and I believe I would have a good career prospects here in near future. 
Likewise, one of the interviewees (Monish) of this study shared that his motive to settle abroad encouraged him to opt for New Zealand. He explained how he analysed the conditions as below:

I was looking for a long term goal because I wanted to settle abroad. As Australia basically changed their rules and regulations, and they were a bit strict and lots of funding is required. They also do not accept the third party sponsors, so I specifically chose New Zealand because of that. I wanted to go for Canada as well but the course structure didn't interest me that much.

However, in the latter part of the interview when asked whether he was satisfied with life in New Zealand, he responded in the following way:

Initially when I came here I was planning to stay but now as the things are moving, I'm planning to finish my studies and move back. So you can yourself presume ... how much I'm satisfied [in New Zealand].

Although this is just one student, his response suggests nevertheless that in order to make New Zealand more attractive to Indian students, there exists a need to know more about their expectations.

This section has reported on factors that influenced the choice of study destination for Indian international students. In brief, the list of priorities consists of quality education, good faculty and courses, image of New Zealand and perception of safety and friendly environment, low cost for study, availability of scholarships, and a few personal reasons such as influence of friends and family ties. It seemed that Indian students had given almost equal priority to the academic issues and to the image of New Zealand. The expectations about career prospects and immigration opportunities also influenced some students. Overall, the survey responses and the interviews indicated that New Zealand was perceived as a more affordable and safe education destination than a few other countries. 


\subsection{Experiences with English Language}

This section presents the background and experiences of these Indian international students with respect to their English language proficiency. Although English is not their first language they are required to use this language continually in academic and social contexts in New Zealand. Moreover, they need to fulfil certain entry requirements in connection to language proficiency before they are offered a place to study in New Zealand.

\subsubsection{English language proficiency: Before arrival to New Zealand}

For the purpose of understanding the students' experiences related to the use of English language, both the survey and the interview included specific questions on English language proficiency in relation to academic and social contexts. In the survey, the students were questioned along three major lines: the use of English language prior to their arrival in New Zealand; their use of English in their academic activities in New Zealand, and the use of language in their social life in New Zealand. The survey indicated that a majority $(80 \%)$ $(\mathrm{N}=105)$ of the Indian international students attempted IELTS and scored an overall band between 7 and 9 in test scores, whereas the university admission requirements specify a minimum overall band of 6 for undergraduate study and 6.5 for postgraduate study (Universities New Zealand, 2012). In detail, out of 84 respondents who attempted IELTS, 67 respondents $(80 \%)$ scored above 7 band. Similarly, five out of nine respondents who attempted TOEFL (internet based) scored between 101 and 111, while the university admission requirements specified a minimum score of 79-80 for undergraduate study, and 90-100 for postgraduate study. Equally, three out of four respondents who attempted TOEFL (computer test) scored 253, 298 and 298 respectively, whereas the university requirement is a minimum score of 233 (The University of Waikato, 2012).

Besides this, the survey revealed that $87 \%(\mathrm{~N}=105)$ of the respondents had their previous education through English medium in India, while only 13\% stated that they had not. When they were asked about the frequency of English language usage in different settings in India, such as home environment, with friends, and at work places, many respondents reported that they had used English in daily life situations particularly in the workplaces. A majority, $73 \%$ of the total of 103 respondents to this question indicated they 'often' used English at their work, 
and a further $16 \%$ indicating they sometimes used English at work, totalling $89 \%$ sometimes or often using English at work. Interestingly, $78 \%$ specified that they sometimes or often used English with friends, and $36 \%$ stated that they sometimes or often used English in their home situations. These figures indicate that these students were using English language in their work and social situations, with some but considerably less usage at home. The Figure 4.2 below demonstrates the detailed responses as found in the survey.

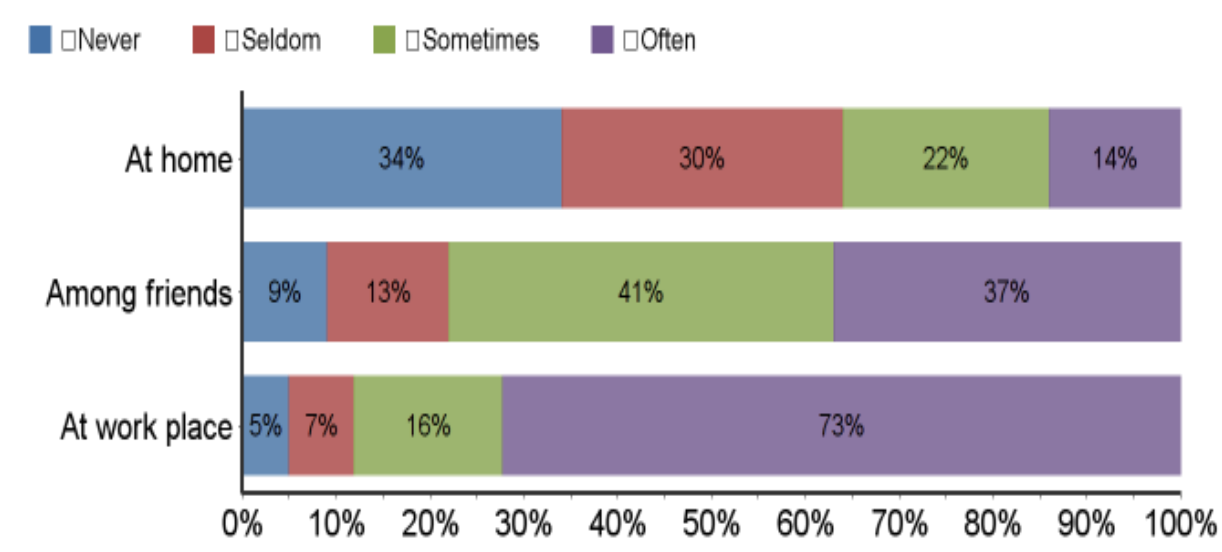

Figure 4.2 - Self-reported frequencies of English usage prior to study abroad

\subsubsection{English language proficiency: Academic contexts}

The survey respondents were asked to rate their own communication abilities in English in academic contexts in New Zealand, for listening, speaking, reading and writing, and overall, on a scale with poor, average, good and excellent categories. For the overall category, 96\% of respondents $(\mathrm{N}=105)$ rated their proficiency as good or excellent. Among the small percentage of respondents (4\%) who rated their English in academic context as average are three male respondents and one female respondent. Two of them were taking undergraduate courses and two were in postgraduate courses other than master's degree. All the four were living outside India for the first time. The high overall percentage indicates the level of confidence of these students about their English language skills. However, for some students self-assessed proficiencies in writing and speaking (productive) categories were lower than those of reading and listening (receptive). Their self-assessment of abilities in individual components on the same scale dropped slightly for writing to $88 \%$. The rest (12\%) included both undergraduate and postgraduate students. Among them there are more male respondents 
$(92 \%)$ than female (8\%), and a majority (92\%) of them are living outside of India for the first time. Remarkably, while two thirds of respondents (67\%) indicating average ability had their previous education in India through English medium, only one third (33\%) had not. In speaking component, among the ten percent of respondents who self-rated their abilities as average, included both undergraduate and postgraduate students, and most of them (90\%) were male and living outside India for the first time. Interestingly, majority (70\%) of them had their previous education in India in English medium and the rest (30\%) in vernacular. The responses received are presented in the Figure 4.3 below.

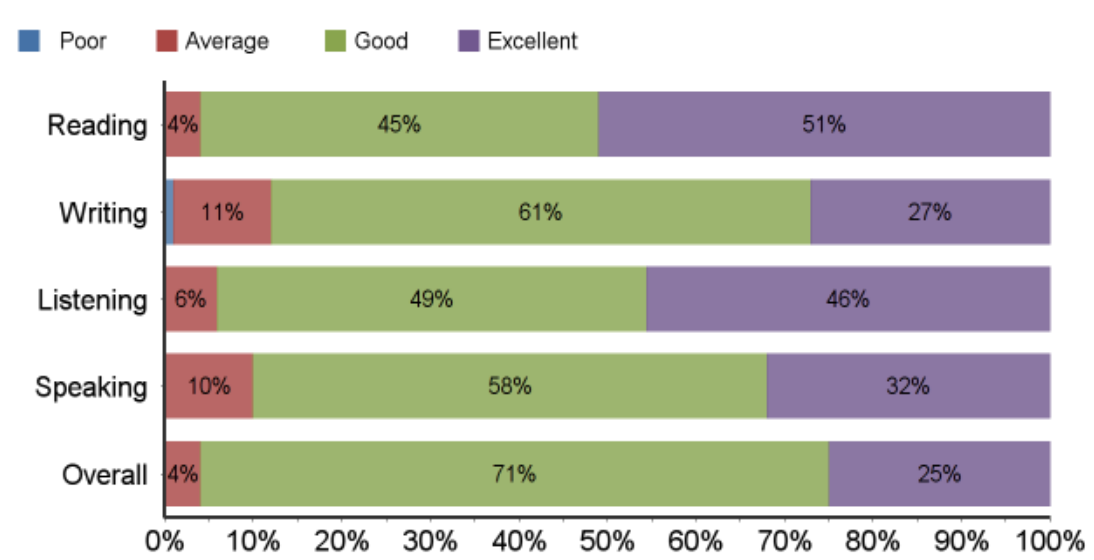

Figure 4.3 - Self-assessment of English language proficiency in academic contexts

The individual interviews with Indian international students encouraged them to reveal past experiences related to this matter. Some participants recalled that they struggled with language issues in their initial period after arriving in New Zealand, but they were able to overcome these challenges rapidly. An undergraduate student shared his experience as below:

Dinakar: I was never familiar with the NZ accent. I heard American and British accents- but not NZ accent which is pretty unheard of in India. Lot of local people couldn't really understand what I was saying. I still think that is still a persisting problem in communication. I have to slow down or I expect them to slow down at times. 
Similarly, two postgraduate students recalled their initial difficulties with language. For one, this was with accents and colloquial language:

Monish: Specifically with one of my tutors, you know, she had a bit of NZ kind of accent, a type of slang thing. Initially for one or two weeks I faced some issues in understanding her, but later on, once things started to roll out, it was fine.

For the other student it was with needing to adjust the speed of his speech to make himself understood:

Pranith: Yes I had. We have a mother tongue influence on our English speaking. So understanding was not a problem for me, but for others it might be a problem for them in understanding as it was influenced by my mother tongue in my initial days here. My speaking was at a higher pace and when I understood it had become a problem for others, I reduced my speaking to a slower pace and also spoke clearly with better pronunciation. Now I have adjusted to the way people speak here.

Some of those interviewed expressed the view that their previous exposure to English language through education, work experience and travelling helped them to deal with these language issues effectively. In this context, it is worth noting that many of the Indian educational institutions offer education through the medium of English. A student studying in such an institution is expected to learn ideas and concepts through using textbooks written in English. At the same time a student is assessed through examinations which would be conducted generally in English. Some institutions even encourage their students to converse in English while at school. Therefore, a person who has graduated from an English medium institution in India is expected to have a good command over the English language. However, one important issue should be noted in this context. Although they have a good foundation in the English language, they may have some difficulties in understanding different accents, idioms and colloquial expressions. Until these students become familiar with these expressions they find it difficult, and once they become attentive to these issues, their adjustment to the conditions becomes easy. For instance, Mahima an undergraduate student in film studies said that, 
Language was not a bit difficult for me, as I've been to an English medium school. But I did notice the difference in accent but it is not too hard to understand. It took just ten days to get acquainted with that.

\subsubsection{English language proficiency: Non-academic contexts}

In the non-academic contexts too a similar pattern was noticed when both good and excellent categories were combined, accounting for at least $86 \%$ of the 105 responses. Self-assessment of writing and speaking skills were slightly lower than for reading and listening. The results suggest that most of the students felt themselves to be proficient in English language and were coping well in non-academic contexts. Among the respondents who self-rated their abilities as average overall, and average in writing and speaking components in everyday communication contexts, are mostly in the age group of $25-29$, first time living outside India, and doctoral students. The response rates are presented in the Figure 4.4 below.

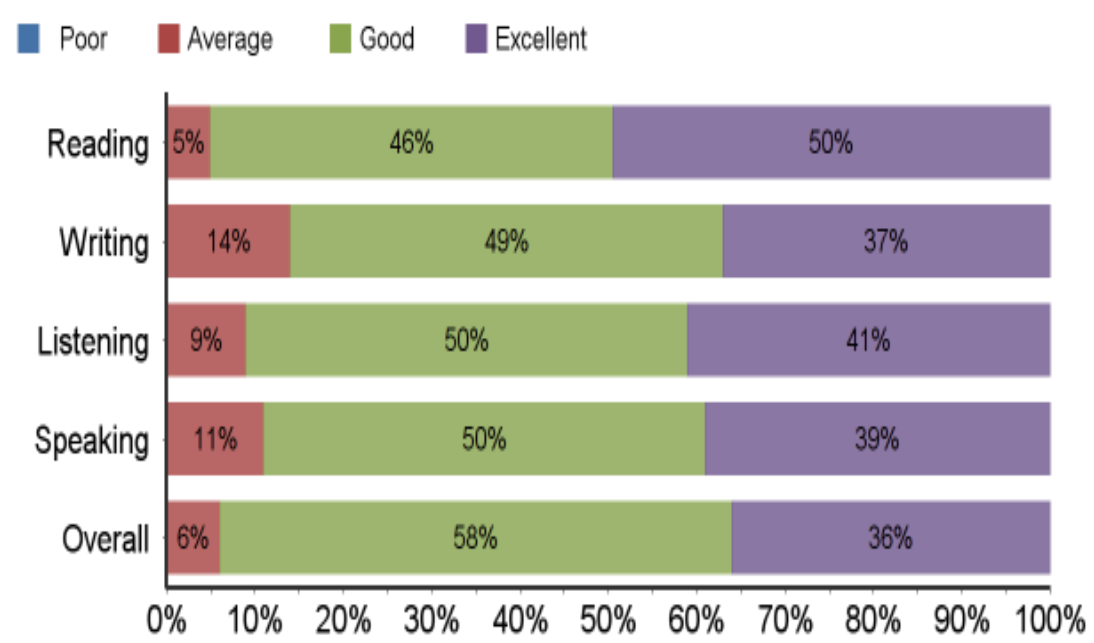

Figure 4.4 - Perceived percentage of communication abilities in non-academic contexts

Currently, international students in New Zealand are permitted to work part-time subject to certain conditions. In the survey the respondents were asked a series of questions on employment. One multiple choice question (Q. 45) asked students to specify their reasons for seeking part-time employment. If they were successful in getting a job, they were asked to specify a reason from multiple options. One of the options was: 'to improve their English 
language proficiency'. In response to this question, only one respondent (out of the total 81 respondents) agreed with this statement. This reinforces the overall sense that these students were satisfied with their English language proficiency.

Similarly, when presented with the statement "My English language ability stops me from making New Zealand friends" (Q. 55.e) and a Likert scale rating of agreement and disagreement, only around $11 \%$ (of the total 102 respondents) either mildly or strongly agreed, with $75 \%$ either mildly or strongly disagreeing. This suggests that the majority of the participants feel that they have adequate levels of language ability to forge friendships with the local people. Figure 4.5 below provides the details of respondents' opinions in this matter.

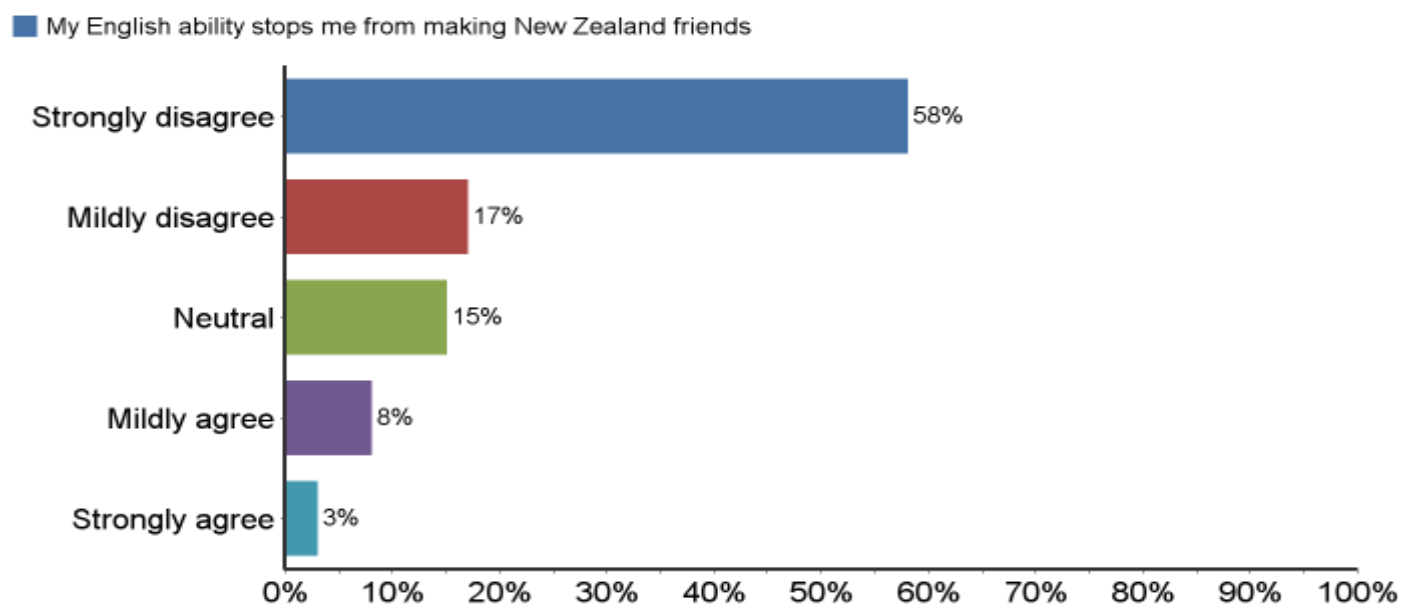

Figure 4.5 - Perceived percentage of language difficulties in making local friendships

To recapitulate, although most of the Indian students experienced little or no difficulties with communication in social contexts, a small number experienced some difficulties during their early days in New Zealand. These included difficulty in following the Kiwi accent, difficulty for others in understanding their Indian accent, having to adjust their pace of speaking, and in understanding the Kiwi sense of humour in conversational contexts. Students reported that coping up with such issues took a few days to few months.

A person who previously worked in an English speaking environment would be less likely to have difficulties in using the language. As noted by the respondents, they have often used English in their workplaces in India. That previous exposure to the language helped them to 
manage in an English speaking environment in the new country. For example, Jasmine who is currently pursuing her postgraduate study had worked previously in a multinational company in Bangalore, India. She narrated her experience in the following way:

I didn't face any problems as such, and one of the reasons could be because I worked with a software company for two years, and my work was all concerned with international clients. So as I already worked with international clients for two years, I didn't face much of a problem initially.

In a similar way a person who had travelled a lot internationally felt that his travelling experience helped him in adjusting quickly to the new environment, especially in terms of oral communication. Victor quickly adapted to the usage of language in New Zealand. When he was asked whether he had any difficulty in understanding local people, he said,

No, not at all...I've travelled a lot and I've been here before. I've friends all over the world.

Thus, compared to other international students from non-English speaking countries, the Indians evidently face less difficulty in communicating in New Zealand. Those who had some previous exposure to the language were able to manage these linguistic issues successfully.

\subsubsection{Strategies applied to overcome language issues}

The interviews provided an opportunity to explore the strategies used by few participants when confronted with language issues. These strategies have been classified into two sub-sections: adjusting their own communication strategies, and seeking help from others.

\section{Adjusting their communication strategies:}

Those participants who were confronted with language issues in New Zealand reported that they generally adapted their own communicative approaches. Such adaptations followed an 
assessment of differences between language usage in India and in New Zealand. For example, Pranith, an Accounting student recognised the influence of his mother tongue on his way of speaking English. He noticed that his speaking of the English language was faster than that of locals, and without using any gestures with hands or appropriate facial expressions while speaking. Therefore, he adjusted to the new environment by speaking in a slower pace and by using gestures to convey his feelings and in passing information to others. He explained his strategy as below:

Pranith: First thing whenever we speak we have to have normal pace in speaking to convey our feelings. Secondly, we should be expressive in passing on information such as happiness while speaking, and thirdly, to listen to them carefully as they speak.

Suresh a Computer Science postgraduate student described how he quickly addressed his initial language concerns:

Suresh: I started watching TV programmes, started listening to the Radio and I made some good friends in my department and it was just a matter of 10 or 15 days.

In a similar vein, Kishore, a PhD student described how he re-tuned his accent and pronunciation in order to communicate better with the local people. He stressed being careful in speaking with local people:

I'll be very cautious whenever I'm speaking to them because I'll try to speak slowly and I always make sure that they should understand whatever I speak, so I just try to speak slowly. I'll always change myself, it is not... how I speak naturally to a guy in India. I'll speak slowly and I'll always change my accent a little so that they will be able to understand. I'll make them comfortable probably that way making myself comfortable. ['them' in this context refers to the local people]. 


\title{
Seeking help from others:
}

Some students sought help from other people while they conversed with or listened to them. For instance, a postgraduate student (Monish) explained that after realising that he had difficulties in understanding the Kiwi accent during conversations, he started requesting others to slow down as they spoke. He described a strategy he used when he could not understand a particular lecturer. His strategy was to overcome his inhibitions and to approach the tutor and to request her to repeat any issues he could not follow. He narrates his story thus,

\footnotetext{
One of my tutors... had a bit of New Zealand kind of accent, a type of slang thing, initially for one or two weeks I faced some issues in understanding her ... I always used to point her ['point her' in this context refers to 'direct attention to'] can you please repeat that, can you please repeat that I don't understand. So next time she would be very slow in telling... me. Sometimes she came personally to me and used to tell me, I meant this I meant that. So that's how I managed.
}

A few participants watched television, movies and other programmes and listened to the radio to improve their English language skills. A postgraduate student (Rashmitha) mentioned that as a home staying student she used the opportunity by conversing with her Kiwi host family. She recollected her memories and stated as below:

\begin{abstract}
My plus point was that I got a home stay arrangement here and I was lucky enough to have a Kiwi lady at home. I used to go back to her and discuss my problems with her and she was very helping in explaining the things. I used to have long conversations with her and that helped me a lot to improve my ability in English language.
\end{abstract}

Although they could efficiently manage the challenging situations, it seemed that they had underestimated the issue of accent; and so they all used their own resources to solve problems; nevertheless, none used the support services in their universities. 


\subsection{Summary}

This chapter initially has highlighted the factors that influenced Indian students in choosing New Zealand as their study destination. The findings of this study indicate that Indian international students studying in New Zealand universities are more likely to be enrolled in postgraduate rather than undergraduate programmes, confirming the trend observed by the Ministry of Education in New Zealand as mentioned in the earlier chapter. Some participants specify that their preference for studying in New Zealand was determined by financial and safety considerations. The doctoral students appreciate the New Zealand government's policy of charging domestic fees for international students in doctoral studies and its policies to encourage research.

The second part of the chapter examined the experiences of the participants with regard to their English language proficiency, such as the usage of language and communication skills before their arrival in New Zealand, and in academic and non-academic contexts in New Zealand. It appears that the participants have demonstrated relatively higher levels of English language proficiency in their language tests to meet the entry requirements of educational institutions in New Zealand. In the same way, a majority of the students self-rated their English language proficiency levels at good and excellent categories, reflecting their higher levels of confidence. Additionally, compared to other international students from non-English speaking countries, for many of them their previous exposure to English language helped them to adjust better to the new environment. Furthermore, in those cases where they faced any difficulties, the strategies applied by these students such as making changes to their communicative approaches and seeking help from others in adverse situations have helped them to manage efficiently their language problems, and ultimately minimised their challenges. In the next chapter findings on their educational experiences along with their perceptions on the facilities and support services in their respective universities will be presented. 


\section{Chapter 5}

\section{Research findings: Academic experiences and the use of support services in their universities}

\subsection{Introduction}

This chapter primarily focuses on the academic experiences of Indian international students in New Zealand. These experiences will also include concerns about their learning and their perceptions of how the New Zealand education system differs from that of India. The latter part of this chapter deals with their perceptions and experiences of the use of different facilities and support services intended for them in their universities.

\subsection{Academic experiences of Indian international students}

This section describes the experiences, challenges and perceptions of the participants relating to their academic study in New Zealand. These descriptions will be followed by an account of different coping strategies applied by the participants as they adjusted to the new learning environment. Subsequently, the participants' suggestions on improvement of conditions related to these issues will be presented.

The responses of the participants under this section are organised into different sub-sections, namely:

- satisfaction with academic life,

- initial impressions of university life in New Zealand,

- different educational system in New Zealand,

- experiences related to educational activities in New Zealand,

- strategies applied to cope with academic issues, and

- $\quad$ suggestions for improving conditions related to academic matters. 


\subsubsection{Satisfaction with the academic life}

The responses to the survey indicated that on the whole Indian international students are positive about their academic experiences in New Zealand, with $48 \%$ being very satisfied. The participants of the survey were asked to rate their level of satisfaction with their academic progress by using a Likert scale with a range of 'Not at all satisfied' to 'Extremely satisfied' (Q. 33). Most of the respondents (87\%) of the total 104 described themselves as moderately to extremely satisfied (See Figure 5.1). However, a few (13\%) of the participants were either only slightly satisfied or not satisfied with their academic experiences in New Zealand.

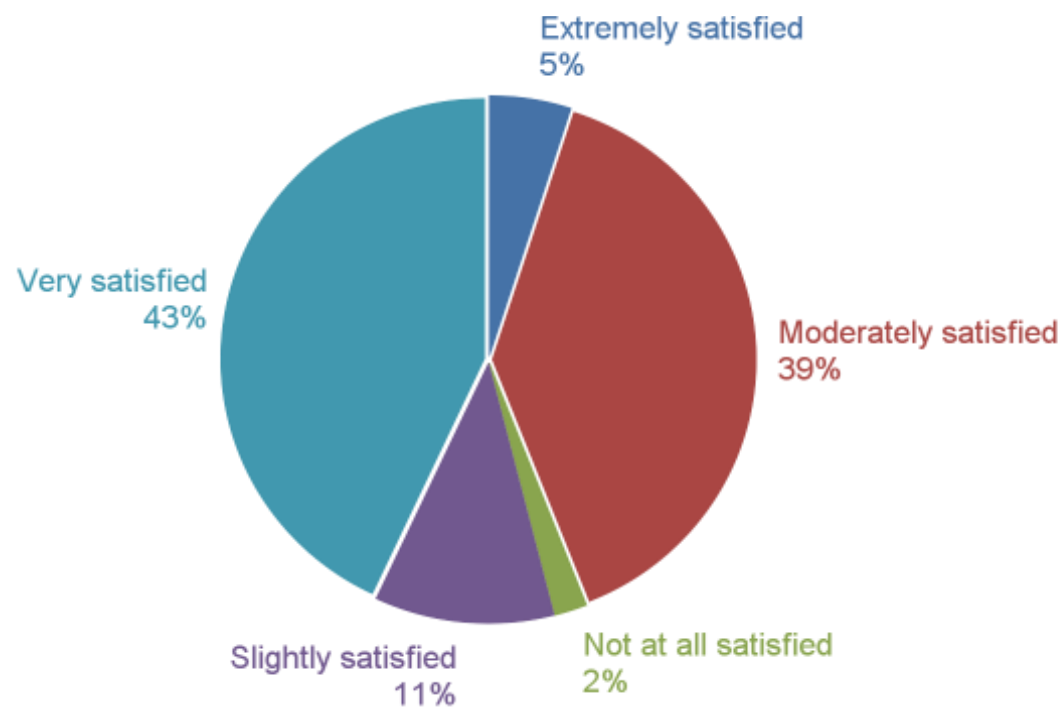

Figure 5.1 - Self-reported satisfaction with the academic experiences

Further to this, respondents of the survey were provided with an option to indicate through an open-ended question why they were not satisfied with their academic progress. Out of the total of 104, only one respondent answered this question. The respondent indicated that the dissatisfaction was due to the ill-treatment experienced because of his/her ethnic background and commented in the following way:

R.83: Some lecturers tend to profile you if you are Indian and tend to treat you according to that first impression. 
While this was the perception of only one student, the findings of the individual interviews conducted at a later stage revealed some personal challenges that they had encountered because of coming from a different academic background. These experiences are narrated below.

\section{Levels of satisfaction among masters and doctoral students}

Fifty-seven percent of the 105 respondents to the survey were postgraduate students, doing either doctoral research or masters by thesis. Another $25 \%$ of the participants reported that they were involved in postgraduate studies, either diploma or Honours, and the rest (18\%) were involved in undergraduate studies. Specific questions were included in the questionnaire for thesis students, because the nature of the supervision experience is different for these students and the requirement of thesis reading, writing, listening and speaking skills is higher than those required of other students who do course work (Q. 35 and 36). The questions were related to different aspects of doctoral or master's thesis work. The participants were requested to indicate their levels of satisfaction with the supervision experience along a measuring scale of 'Not at all satisfied' to 'extremely satisfied'.

The results shown below in Figure 5.2 indicate a high level of satisfaction of the respondents. Seventy percent of the 56 respondents were highly satisfied with the academic guidance provided by their supervisors. However, master's students were less satisfied (57\%) compared to doctoral students (77\%). Likewise, $77 \%$ of the respondents rated themselves as extremely or highly satisfied with the provision of information and support provided for the use of library resources and services. The satisfaction rate among females is a little higher $(85 \%)$ than the satisfaction rate of males $(74 \%)$. In the same way, the satisfaction rate of doctoral students $(92 \%)$ is higher than the satisfaction rate of master's with thesis students (52\%). Similarly, 56\% of the participants were very or extremely satisfied with the opportunities for skills development required for their thesis work. Within this category the rate of satisfaction with males $(77 \%)$ is higher than the females $(53 \%)$. As mentioned in the above categories as well, the satisfaction rate of Master's students is much less (24\%) than the doctoral students' (75\%). A slightly lower proportion (54\%) of the participants stated that they were given opportunities to participate and present at research seminars. In this category as well the satisfaction rate of master's students $(24 \%)$ is lesser than that of the doctoral students $(71 \%)$. However, it is notable 
that only $41 \%$ of the respondents stated that they had been provided with clear information on their rights as doctoral/thesis students, and on provision of different scholarships or funding opportunities. Overall, the master's students expressed their less satisfaction compared to doctoral students related to their academic aspects.

\begin{tabular}{|c|c|c|c|}
\hline Moderately satisfied & Extremely s & tisfied & \\
\hline Information on what is required of you as a doctoral/thesis student & $36 \%$ & $38 \%$ & \multirow{2}{*}{$\begin{array}{l}9 \% \\
11 \%\end{array}$} \\
\hline Access to information on your rights as a doctoral/thesis student & $34 \%$ & $30 \%$ & \\
\hline Opportunities for scholarly interactions with other postgraduate students & $7 \% 13 \%$ & $34 \%$ & $16 \%$ \\
\hline Opportunities to interact with academic staff other than supervisor(s) & $13 \%$ & $25 \%$ & $22 \%$ \\
\hline Opportunities for skills development e.g. Writing proposals & $7 \% 11 \%$ & $36 \%$ & $20 \%$ \\
\hline Opportunities to participate and present at research seminars & $20 \%$ & $29 \%$ & $25 \%$ \\
\hline The academic guidance provided by your supervisor(s) & $13 \%$ & $34 \%$ & $36 \%$ \\
\hline Information and support provided on using Library resources & $25 \%$ & \multicolumn{2}{|c|}{$52 \%$} \\
\hline Information and support provided by Information Technology Services & $9 \% 7 \% \quad 16 \%$ & $27 \%$ & $32 \%$ \\
\hline Information provided on different scholarships /Funding opportunities & $24 \%$ & $25 \%$ & $16 \%$ \\
\hline
\end{tabular}

Figure 5.2 - Levels of satisfaction with different aspects of doctoral/thesis work

The detailed view of the respondents on the above issues indicated that they were least satisfied with the provision of information on different scholarships or funding opportunities, with $40 \%$ of the total 56 respondents rating their level of satisfaction at either 'not at all satisfied' or 'slightly satisfied' categories. Similarly, $20 \%$ of the respondents noted their dissatisfaction under these two categories with regard to the provision of opportunities related to scholarly interactions with other postgraduate students. Closer to this, $18 \%$ indicated that only limited opportunities were available to them for getting scholarly interactions with academics other than their supervisors. Likewise, $18 \%$ of the respondents were dissatisfied with the level of opportunities they had for academic skills development. A full list of the participants' levels of satisfaction on different academic aspects can be viewed in Appendix 6.

Besides this, the participants were given an option in the questionnaire to specify if they were not satisfied with any aspect of being a postgraduate thesis student. Out of the 19 responses received, four comments were related to the provision of information on scholarships or 98 
financial support. Two comments were about perceived discrimination, one comment was about dissatisfaction related to the academic staff. Other respondents did not have any complaints. Among the three types of grievances noted above, a few examples are provided below:

A comment related to lack of information on the scholarships:

R.54: Not much information is readily available with respect to funding and different scholarships for international students...

Another comment related to perceived discrimination:

R.106: As far as my experiences are concerned, I had lot of bitter experiences. I felt the treatment that was given to me was very biased and I felt it was a sort of mild racism. I do not want to generalise, but I felt so. People here do not respect your academic qualifications or your knowledge. I would expect few similar ways of treatment anywhere outside your own country among white people, but this is my first experience.

Another respondent's view on perceived discrimination:

R.108: During my Honours degree, I had to deal with racism and harassment from my programme director and another lecturer. It was a traumatic experience, and I still have not understood how international students can be treated as commodities that bring in funds for the university.

In connection with the dissatisfaction with academic staff, one of the respondents a master's degree student commented like this:

R.49: The teaching faculty in the university is very poor. They have got more visiting lecturers than permanent full time Professors. This is badly affecting the 
students since in every term there is a new Professor who steps in without having or with little experience.

It is interesting that in spite of the fact that all the universities provide detailed information on scholarships or different funding opportunities through university websites, some respondents reported being unaware of such information. One possibility is that the students were dissatisfied with the limited number of scholarships available rather than the lack of available information. Likewise, the comments on racism did not include any descriptions of the incidents other than the perceptions of the respondents.

\subsubsection{Initial impressions of the university life in New Zealand}

When interviewed, the participants were asked about their initial impressions of university life in New Zealand. The purpose of this question was to explore their reflections on a new academic environment. Not surprisingly, the participants had diverse experiences and perceptions of their new academic environment.

A few remembered the excitement of arriving in a foreign land and being impressed by the hospitality and support received from the university. The main difficulty appeared to have been with understanding the unfamiliar local accent.

One undergraduate student was confused to find two different campuses of the same university in the central part of the city, a feature uncommon in India. Another student was disappointed with his university's urban location and the absence of a large exclusive campus in a peaceful environment. One was surprised by the large lecture halls. The students' initial impressions varied from amazement to bewilderment.

\subsubsection{Different educational practices in India and New Zealand}

The survey posed a few questions to explore the learning experiences of Indian international students, and the respondents were asked to rate the difference between educational practices in India and New Zealand. The scale ran from 'very different', 'different', 'similar', 'very 
similar' and 'unsure', with items related to academic workload, teaching practices, assessment procedures, interaction between students and lecturers, and interaction with other students in their classrooms. The data collected, as shown in the Figure 5.3 below, indicate that in a number of areas the respondents felt that the two academic systems were 'different' or 'very different'. The combined percentages of these two categories were:

- academic workload $85 \%$ (of the total 105 respondents),

- teaching practices $89 \%$,

- assessment procedures $86 \%$,

- communication with lecturers $87 \%$, and

- interaction with other students in the class $73 \%$.

Thus, the findings highlighted that these Indian international students encountered a new and very different university system and practices which they were unfamiliar with.

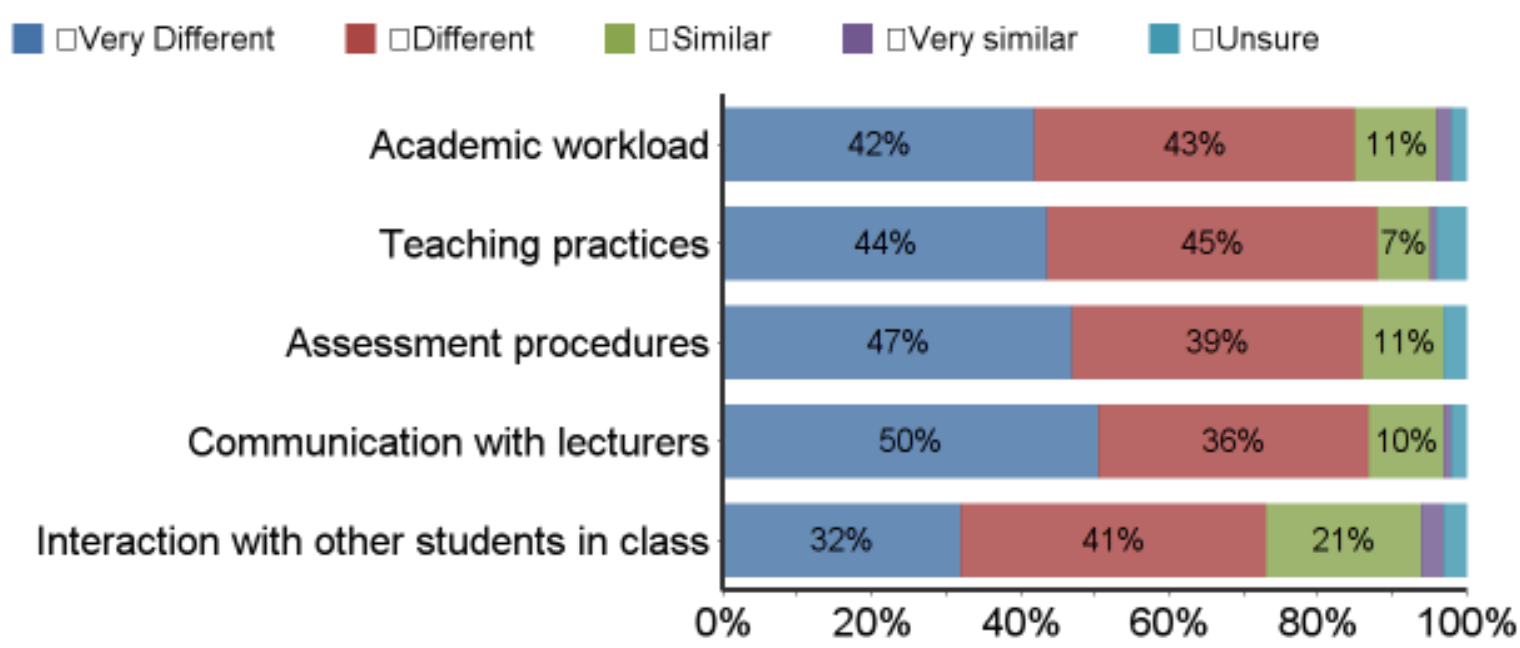

Figure 5.3 - Differences in educational practices between India and New Zealand

Furthermore, the respondents of the survey were asked an open-ended question to identify three positive and three challenging issues related to their educational experiences. Ninety five students responded to these questions and indicated what they enjoyed most in their academic experiences, as well as the challenges they encountered in their academic life. All the positive and challenging experiences narrated by the respondents were coded and organised into 
different categories. Some of the significant categories are provided in the table 5.1 below and the detailed list of data can be viewed in Appendices 4 and 5 .

Table 5.1 - Most enjoyed and challenging academic experiences reported by the participants

\begin{tabular}{|c|c|c|}
\hline Category & Sub-categories & $\begin{array}{l}\text { Frequency } \\
\text { count } \\
(\mathrm{N}=362)\end{array}$ \\
\hline & \multicolumn{2}{|l|}{ Most enjoyed academic experiences } \\
\hline $\begin{array}{l}\text { Learning } \\
\text { approaches }\end{array}$ & $\begin{array}{l}\text { Practical approaches, giving presentations, } \\
\text { participating in discussions and interactions, critical } \\
\text { thinking, group works, study tours }\end{array}$ & 57 \\
\hline Teaching staff & $\begin{array}{l}\text { Good, friendly and encouraging teachers or } \\
\text { supervisors }\end{array}$ & 36 \\
\hline Resources & $\begin{array}{l}\text { Library, laboratory and infrastructure, online journals, } \\
\text { collaboration with other researchers }\end{array}$ & 35 \\
\hline $\begin{array}{l}\text { Friendly } \\
\text { environment }\end{array}$ & Friendly environment, peer support, casual classrooms & 24 \\
\hline $\begin{array}{l}\text { Course work } \\
\text { material }\end{array}$ & Study material, course structure, optional workload & 22 \\
\hline Teaching & $\begin{array}{l}\text { Different approaches in teaching, including interactive } \\
\text { lectures and use of technological resources in teaching }\end{array}$ & 18 \\
\hline $\begin{array}{l}\text { Assessment } \\
\text { procedures }\end{array}$ & $\begin{array}{l}\text { Different assessment procedures including } \\
\text { assignments and less focus on written examinations }\end{array}$ & 16 \\
\hline \multirow[t]{2}{*}{$\begin{array}{l}\text { Personal and } \\
\text { other factors }\end{array}$} & Freedom, new educational system, minimum stress & 48 \\
\hline & \multicolumn{2}{|l|}{ Most challenging academic experiences } \\
\hline $\begin{array}{l}\text { Assessment } \\
\text { procedures }\end{array}$ & $\begin{array}{l}\text { Writing/completing assignments, assessment styles, } \\
\text { too much research involved and completing the } \\
\text { assignment as per schedules }\end{array}$ & 22 \\
\hline Academic issues & $\begin{array}{l}\text { Giving oral presentations, academic writing, lack of } \\
\text { resources }\end{array}$ & 17 \\
\hline Language issues & $\begin{array}{l}\text { Lack of speaking skills, understanding language/ kiwi } \\
\text { accent, coping with language levels }\end{array}$ & 11 \\
\hline $\begin{array}{l}\text { Teaching and } \\
\text { learning } \\
\text { approaches }\end{array}$ & $\begin{array}{l}\text { Critical thinking, independent learning/problem } \\
\text { solving, group projects }\end{array}$ & 11 \\
\hline $\begin{array}{l}\text { Educational } \\
\text { system }\end{array}$ & $\begin{array}{l}\text { Education system, team dynamics, pattern of exams, } \\
\text { course work }\end{array}$ & 11 \\
\hline Academic staff & $\begin{array}{l}\text { Perceived discrimination, interacting with teachers and } \\
\text { supervisors, questioning the lecturers, perception of } \\
\text { average lecturers, and differences among the } \\
\text { supervisors }\end{array}$ & 6 \\
\hline
\end{tabular}




\begin{tabular}{|l|l|c|}
\hline Racism & Racism, arrogance, personal vendetta & 3 \\
\hline $\begin{array}{l}\text { Other factors - } \\
\text { Personal }\end{array}$ & Culture shock, isolation, shyness, loneliness, and so on & 17 \\
\hline $\begin{array}{l}\text { Other factors - } \\
\text { Social }\end{array}$ & Finance, weather, earthquakes and so on & 8 \\
\hline
\end{tabular}

Since the survey data indicated the existence of differences as well as many challenges, each academic area was further probed through the individual interviews. During the interviews, participants were asked whether or not they anticipated differences before coming to New Zealand. Furthermore, students were asked to share their encouraging as well challenging learning experiences in what was apparently an unfamiliar academic system for them.

The responses of the participants under this section are organised into six different components, namely:

- different approaches to learning: theoretical and practical aspects,

- independent learning,

- choosing the degree and course planning,

- continuous updating of curriculum,

- the student-teacher relationship, and

- use of technology and internet in the teaching and learning process.

\section{Different approaches to learning: Theoretical and practical aspects}

When asked about whether or not they found any differences in the approaches to learning, an undergraduate participant of the interviews Dinakar explained his perception. According to his view, in India, usually the emphasis falls on learning a particular pre-set curriculum and involves memorising the concepts found in the set textbook for a particular course. In his own words:

There [in India] it was a set prescribed text book and you just have this much of the portion covered in a semester... 
The educational approach in India is usually grounded in acquisition of theoretical knowledge, rather than critically analysing it or applying it in a practical field. Then, examinations test the memory of the student. A female undergraduate student (Mahima) commented on the dissimilarities in assessment procedures. She observed:

There is a lot of difference. .... It's more analytical here and you have to think very critically. It's not just 'mugging up'3 and writing the stuff. ... You have to do your essays and initially I was very nervous because I haven't done anything like that back home in India.

By contrast, in New Zealand a number of participants perceived that studying involved a much more practical approach. Dinakar went on to say:

[In New Zealand] ...there are no prescribed text books and it's more like reading a lot of articles and journals and stuff which was something that I was not used to back in India. In India it is much more theoretical and here it is more like practical.

On the same issue Vineel, a student pursuing his postgraduate studies, shared his experiences:

More of practical work in New Zealand, assignments rather than memorising the book, going through the articles, expressing the ideas whatever you feel like and new innovations in the study. In India it is more memorising and rewriting.

Moreover, students felt that academic learning in New Zealand involved applying knowledge they learned in the classroom to the assignments. Interestingly, one student (Jasmine) reported that she wanted to study overseas because of this practical component of academic learning. She illustrated her views in the following way:

For example, [in India] we were given books. We have to grab everything. We have examinations on paper. We have to write what we learn in the class. But here it is something different. What I have noticed was we have internal assessments and

\footnotetext{
3 'mugging up' means study or review intensely (mugging up, 2014)
} 
more of the grades are actually, I mean, they focus more on presentations, discussions and we do get grades for taking part in those discussions, but it never actually happened in India. So I can tell you an example... Here if you learn of computer course they give us more of practical knowledge with... laboratories and with...more discussions. We were given more hands on experience. So I believe that's the practical knowledge that I am talking about.

Similar to this view, Rajasekhar a student who was involved in his doctoral study in physics, described the theoretical and practical approaches in India and New Zealand respectively. In his perception, in India emphasis was given to theoretical knowledge and in New Zealand importance was given to the practical approaches and students would learn new things by applying their knowledge by doing things practically. He describes the differences:

Even though I did my masters in one of the best places in India, there was a lot of less exposure for application of your skills or theory. What we did in masters actually we studied a lot of theory and solve really complex problems...but little emphasis on ... practically applying those skills. You know or you learn a particular theory, but then, how do you apply this particular theory to a particular situation. Those are the skills I didn't get to learn in India and here that's what a lot of emphasis people put on. You learn a particular theory but you don't have to go in depth there perhaps... You learn a theory and you are very confident that you understand that theory, but you never understand a theory unless you apply it. Here you learn a theory and they put a lot of emphasis on problem solving... That's actually a major difference.

Interestingly, as a deviation from the above views, one student who is currently doing his postgraduate studies in Accountancy (Pranith), shared a different experience. He explained that he used to have a practical approach in India whereas in New Zealand he learnt the theoretical part. He believes that the university in New Zealand helped him by equipping him with theoretical knowledge. These observations indicate that Indian students in New Zealand get exposed to an educational system which appears to be very different from what they previously experienced in their home country. 


\section{Independent learning}

Participants of the interviews explained another educational practice which differed significantly from those prevalent in India, namely the 'independent learning', a process expected of students in New Zealand. Rajasekhar who did his master's degree in a highly regarded central university in India, explained that in India his supervisors closely monitored the students and guided them to accomplish the given tasks. Contrary to this, in New Zealand supervisors provide guidance to a certain extent and expect the students to do their own. The same student later commented that such independent learning helped him to gain confidence in himself. Besides this, he added that this independent approach helped him in his personal life as well.

However, another student who is pursuing his doctoral studies in Health Sciences said that he was not aware of any differences in the educational systems between India and New Zealand. Interestingly, despite not seeing the difference, this student indicated that he was not aware of the expectations of 'independent learning' prior to his studies in New Zealand.

\section{Choosing the degree and course planning}

Another difference in educational practices where a few Indian international students struggled was what they perceived as 'designing their degree' by selecting appropriate courses. In New Zealand while doing a degree with course work, other than doing a degree by thesis, one has to first decide on the desired qualification among the general and specialist degrees. For instance, some Indian students have enrolled in general degrees such as B.A (Humanities) and Postgraduate diploma courses in Science. Some others have enrolled in specialist degrees such as Accounting or Engineering. This choice may be based on one's career aspiration or on one's own interest. Specialist degrees, such as Accounting and Engineering are mostly structured with some courses being compulsory, and with clear pathways, while general degrees such as Bachelor of Arts and Postgraduate Diploma typically have flexible options where students need to select courses consisting of majors, minors and electives. They have to plan their study over a period of time in accordance with the stipulated principles. 
Some students find difficulty in choosing courses or 'designing their own degree', as it is not a familiar concept to them. In India, generally for all the degrees irrespective of their being general or technical (professional courses), there is little or no choice within the degree programme. They are generally provided with a pre-designed curriculum with specified courses and students are expected to fulfil the set requirements for the degree. ${ }^{4}$ Sometimes in a few circumstances there may be a choice of courses for that degree, but it would be limited to a few courses or in selected institutions. As a result, some newly arrived Indian international students find it difficult to comprehend this particular educational practice. Dinakar, an undergraduate student described the difficulty he had in the following way:

\begin{abstract}
In India it is just a set programme and you are supposed to complete these papers in that programme. Selecting papers is not something that you are used to and you don't know how to design your degree and designing your degree is a new thing completely. It is a nice option but you still need help to understand how you could do it effectively and without wasting a lot of time on papers that will probably not even be contributing to my majors.
\end{abstract}

Prior to coming to New Zealand some participants were not aware that they had to choose their own courses. As they did not know, they could not also take advantage of the system. However, after realising the flexible nature of this system, the participants began to feel that it was after all a good thing for them to be able to choose papers according to their interests. For example, Monish who is pursuing his postgraduate studies with course work in Human Resource Management shares his experience in the following way:

The main thing which concerned me was that there was no one to guide me which course to take. Actually, I went to the course administrator and she just gave me this course and that course, ok, I'll enrol you in these two courses. So being an international student I didn't know that I had an option to select my courses on my

\footnotetext{
${ }^{4}$ For instance, the three year course structure of BCA (Bachelor of Computer Applications), an undergraduate course offered from one of the Indian university can be observed through the URL provided in the reference (Acharya Nagarjuna University, 2012).
} 
own. I thought, you know, they are senior persons so they themselves selected. There was no one to guide me. Now I know...I have an option so that I can change my papers now. So I'm planning to do the same in my second semester because she assigned me all the courses for my first as well as for second semester. I may have selected the same courses if I had to decide, but I was not informed that I can select on my own. That was the main thing.

\section{Continuous updating of curriculum}

One of the interviewee (Dinakar) pursuing his undergraduate studies in Humanities emphasised that in New Zealand the curriculum is brought up to date continuously on the basis of recent scholarship, whereas in India one tends to have a curriculum that runs for a certain period of time. Usually in India, in accordance with the developments and changes in the subject field, a panel of experts in the subject area would be constituted and make proposals on the required revisions of the curriculum. Later, with necessary approvals from the competent academic authorities the proposed new curriculum would come into force from a specific date and would continue until the next revision takes place. ${ }^{5}$ However, although this practice of continuous updating of curriculum in the New Zealand education system is unfamiliar to the participant, he welcomed the practice by commenting in the following way:

Here things get updated every two weeks or so [participant's perception] and I think it is a nice thing and it's not the age-old stuff that we are studying.

\section{The Student-teacher relationship}

Many of the participants in our study are of the opinion that the student-teacher relationship is different in New Zealand as compared to the Indian situation. According to them, teachers here are more accessible, cordial, friendly, funny, frank, and encourage independent learning. Most teachers were seen as guiding and helping students and letting them know what was expected

\footnotetext{
${ }^{5}$ An example on these curricular revision issues from one of the highly regarded universities in India may be viewed through its URL provided in the reference (University of Pune, 2011).
} 
of them. By way of contrast, participants shared their experiences of student-teacher relationship in India. These included: respecting the teacher, being afraid of the teacher, the teacher as equivalent to God, and a student not expected to argue with or question the teacher. It sounded like formal conventional relations still existed between students and teachers in India.

Due to these differences Indian students in New Zealand enjoyed their learning experience, as they had the freedom to express their thoughts and opinions. The following comments expressed by the participants based on their experiences and perceptions illustrate this point. One student particularly appreciated the ease of access to the lecturers:

Dinakar: Here student teacher relationship is much more cordial than in India I think. Here there is a freedom that you can go visit lecturer at any time and they are easy to talk to. It's a much better relationship definitely and something that I enjoy.

Others, such as Monish, relished the encouragement of independent thinking:

Monish: The thing which I liked most as compared to India is that you have your own freedom to express your thoughts...sometimes there are some. I will not say all of them, but there are some lecturers who are quite helpful but not all of them. They definitely help you out as compared to India. In India too, yes I had some lecturers who are very helpful, but here they will let you know what exactly they need. And how you should approach, they don't spoon feed you. That actually gives you time to improve and time to focus on yourself and actually think out of the box.

However, the task of moving from a teacher directed system in which the teacher was the authority, to one with a greater emphasis on independent learning, proved to be difficult for some students.

Suresh: This system is different from India. In India your teacher would tell you to do this and this, and you would do it. If you [get] stuck somewhere the teacher is 
supposed to give you the answer. Here the teacher wouldn't really give you the answer, but he would tell you where you could find the answer... The transition was very painful coming from a country where we were spoon-fed doing everything to doing things by yourself. But I've adjusted.

While the majority of participants provided a positive picture, a few also shared their less than satisfactory experiences with teachers/supervisors. Pranith, a master's student, explained that he had a teacher who did not care whether or not his students were following his lectures.

Here a few lecturers ... teach something which is irrelevant and they don't see whether the student understand their teaching or not... One teacher teaches we don't understand what he teaches. For example, in my finance class the teacher... teaches something...which I don't understand. But he never cared whether I understood or not.

Similarly, a doctoral student (Kishore) referred to his perception of the casual approach of his supervisors in fixing schedule, arranging meetings, or in providing feedback, which hampered his study in the initial days. He narrated his strained relationship with one of his supervisors:

...so, with the second supervisor I don't meet often because sometimes, I was not happy with the way he speaks to me. I told him personally I'm not happy... because you speak like this...it puts me off and I don't get along well I told him. I just asked him what your problem is. I just asked him if you have any general problem with Indians...because I have seen him treating not only me... some other Indians too, we were a bit hurt most of the times...

In the case of another interviewee, Rajasekhar, even though his relationship with his former supervisor was not satisfactory, his experience with his later supervisor turned out to be positive. He commented on his experience in the following way:

I had actually two supervisors in my PhD. My initial supervisor left after a year for a new job. So I had to choose a new supervisor. My first supervisor was a bit pressing. He was a bit demanding. The rapport was actually not that great. Once 
I actually chose my other supervisor after he left and with that second supervisor, I have a very good relationship and he is very friendly, enthusiastic, encouraging, and he is not very pressing. He gives you a space to do things. I've very good relations with him. He gave me my own space and enough time to groom myself.

The above experiences of Indian students point out that even though a remarkable number of Indian students expressed satisfactory relations with their teachers or supervisors, there are some students who had unpleasant experiences. Though these experiences are less in number, they warrant nevertheless special attention by the concerned authorities.

\section{Use of technology and internet in the teaching and learning process}

One aspect that a number of participants identified as dissimilar to their experience in India was the use of technology and internet in educational environment. University students in New Zealand are expected to do research for their assignments, either by using library resources or through browsing online databases the libraries subscribe to. The internet is an essential tool in the learning activity. In India preparation for an examination would typically involve studying from text-books or other printed materials. Given the differences between the two educational systems, some participants felt that new international students required time to acquire the necessary skills. A recently arrived postgraduate student's (Pranith) experience highlights the steep learning curve involved in coming to grips with the online components of study:

...I'm not that used to have technical learning. Student should be given an opportunity on how to get used to this technical knowledge...I...never used this elearning stuff, because we used to follow text-books. First few weeks were difficult for me because it was quite impossible for me in the first few days to go to Blackboard and learn and study using the laptop. It took me a month to cope up with that environment because here it is compulsory.

Although it cannot be generalised, yet Pranith's experience suggests that there is a need for institutions to understand the cultural backgrounds of these students to evaluate their technical capabilities. Other than this, these students may be provided with adequate time to get familiar 
with the use of technology and to provide necessary support to improve their skills in using the tools. The interviewee further explained the limited access and usage of internet, highlighting the situation in his previous education system in India in the following way:

As the international students come from different colleges and backgrounds ... international students should be given an opportunity to understand this and get to familiarise with the tech usage...in India we never used internet at college. We don't have a website for our college in India. We use computers once in a while. Here computer usage has become mandatory. So they should give students a time to come up with this culture...we are not used to this tech...

In the subsequent stages of the interview the same participant provided some more insights into the circumstances in India related to this issue.

...I have a friend who is a good student with better knowledge than me, but lacks computer knowledge, which is common in India. Indian families still think that computer is a distractive object. A father in India still thinks whether to buy a computer for home or not because he feels that computer distracts a student, it may be funny for some people. It wasn't necessary in India. The only reason we use computers in India is like once in a while. My teacher uses it for a presentation or we will do a presentation in computer which is a rare thing. Except for science students other students never use computers because we only use books, books, books. Here irrespective of subjects all students use computers.

In New Zealand academic study involves participation in a variety of educational activities such as lectures, tutorials, workshops, individual research in library, in laboratory, through internet, meetings, attending seminars, and giving oral presentations. Some, but not all courses, will have examinations. However, when compared with New Zealand, in India typically academic study involves limited application of learning methods beyond lecturing by the teachers, and taking examinations at the end of the year by the students (Acharya Nagarjuna University, 2012; Clark, 2006). As our study shows, some Indian students find it initially difficult to adjust to these differences. 


\subsubsection{Experiences related to educational activities in New Zealand Universities}

As shown above, there are several aspects of the New Zealand educational system that are different from the Indian system. Specifically, according to the perceptions of the respondents of the survey, in the aspects of academic work load, teaching practices, assessment procedures, communication approaches with their lecturers, and interactions with other students in classes.

In the midst of the different and unfamiliar academic culture of New Zealand, the participants were asked to indicate their levels of difficulty in a range of 'Not at all' to 'extremely'. Surprisingly, the findings from the 104 survey responses suggest that a majority of Indian international students have little difficulty in most of their educational activities. It appears that in a majority of cases, although the respondents had to undertake very different kind of educational activities than what they did in their home country, their English language proficiency and individual abilities helped them to accomplish their academic tasks. However, a minority of respondents also indicated that they faced difficulty in certain areas, such as, writing assignments (36\%), thinking critically (24\%), completing assignments on time (21\%), working on group projects (21\%), giving oral presentations (25\%), managing workload (25\%), and studying in a different educational system (24\%). A few respondents indicated that these activities were not applicable to them, because of their nature of study (e.g., thesis writing). Figure 5.4 below shows the percentages of individual categories of different levels of difficulty. A detailed list of percentages can be viewed in Appendix 8. 


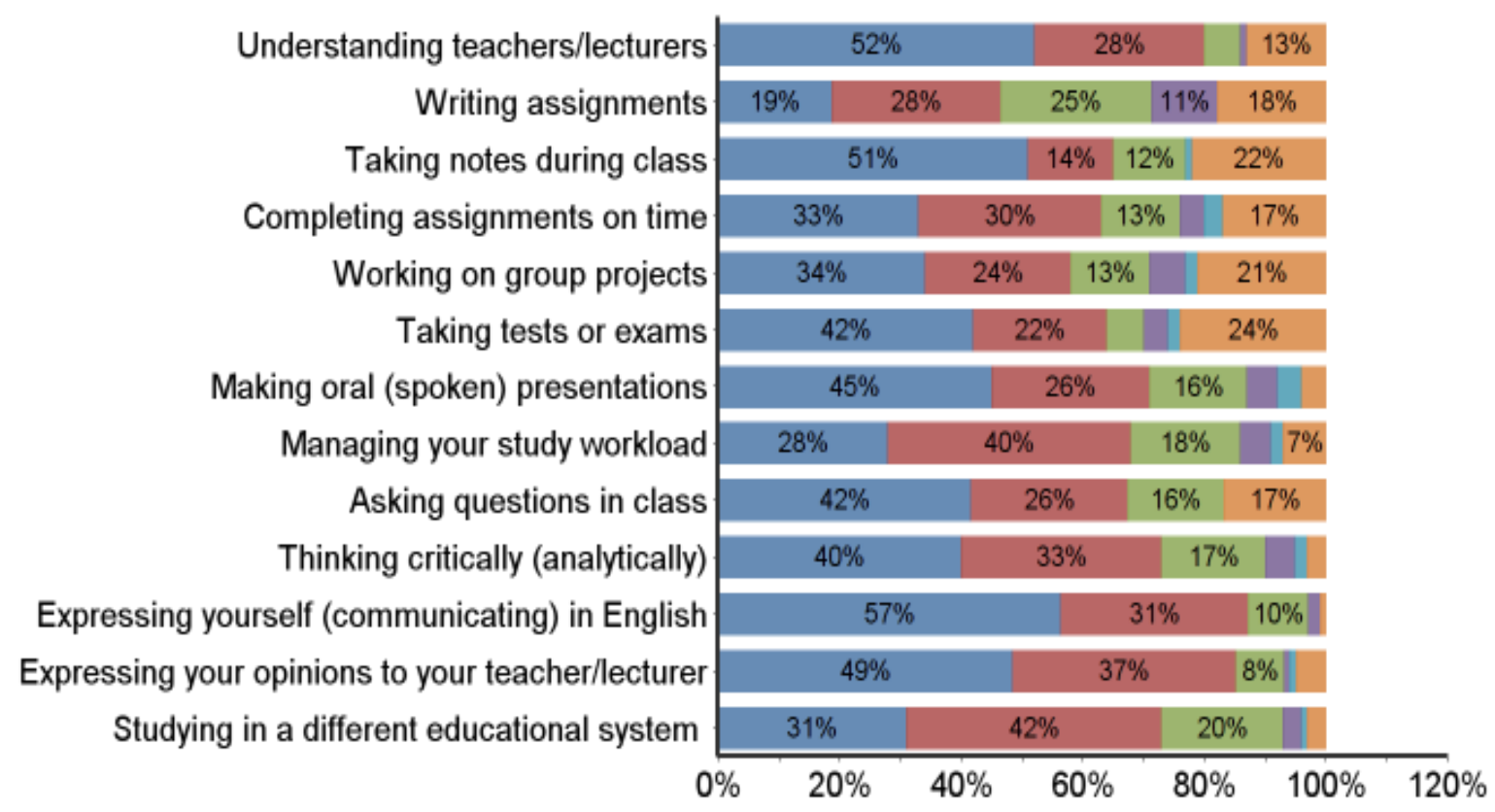

Figure 5.4 - Participants' levels of difficulty with educational activities in New Zealand

The individual interviews also reflected this trend, as most of the responses were positive. The majority of students confirmed satisfaction, narrated their unique experiences and recounted the factors that helped them to achieve their academic goals. One postgraduate student in a management course thought that the small number of students in his class helped him to participate actively in the classroom.

\begin{abstract}
Monish: Participation in one class it is good because the number of students is very small. It was around 8 students most of them were very experienced, they have some seven years or ten years of work experience. In the second class there are some people who had 35 years of experience as well. So it was a nice mix and match. In the beginning ... all were not comfortable but as we moved on and had our presentations and group discussion, then things started to move on, we started discussing a few things with each other, then it was good.
\end{abstract}

Another female postgraduate student in the same management course was appreciative of the experience in New Zealand. She explained that encouragement from tutors helped her to 
participate in classroom discussions and presentations. She made comparisons with her previous educational experience in India in the following way:

Jasmine: It's fantastic I believe. I told you there are so many differences in education systems of India and New Zealand, one being the practical knowledge, the other is there are lots of discussions. I didn't feel that kind of knowledge being important to me in India. The tutors actually here encourage students to take part in discussions. We have lots of presentations and yeah, it helps a lot...., I mean, they focus more on presentations, discussions and we do get grades for taking part in those discussions, but it never actually happened in India......

In a similar way, another postgraduate student (Pranith) was very excited to share his experience of opportunities to speak in class. He asserted that such opportunities helped him to gain confidence. He considered that his study in New Zealand really benefited him mostly in that aspect.

They give an opportunity to speak in front of 50 students. First time I've feared because I have never spoken in front of a class of 60 students. But the second time onwards I felt confident. So now I don't feel fear to give a speech in front of 100 or 1000 guys. That one I learned a lot. That one is the most important thing I have learned here. Never fear in front of a crowd. ... this college gave me that 'confidence'. I believe that the college gave me that 'I can'. In India 'Can I' was the question. But now 'I can'. This college taught me many things it gave me confidence, 'can I' became 'I can'.

However, out of the fifteen interviewees six of them admitted that they encountered difficulties in New Zealand because of different academic culture. Two undergraduate interviewees explained their difficulty with assessment procedures such as writing assignments and keeping

\footnotetext{
6 The word 'college' in India refers to higher educational institutions which may offer courses up to postgraduation level apart from universities (Acharya Nagarjuna University, 2014). So the participant used the term for a New Zealand university. The term should not be confused with 'college' in New Zealand.
} 
with the schedule. One postgraduate diploma student commented that the expectations such as structuring an essay were unfamiliar to them, as they were not given any guidance on these issues during their study in India. Another postgraduate diploma student explained how his inadequate language proficiency obstructed his effective classroom participation. Two other postgraduate students (one master's degree and another doctoral degree) stated that their difficulty was limited to initial days and that was a very hard and painful experience.

Since written assignments are unfamiliar phenomena for these students, some of them seemed baffled initially with this aspect of their academic study. One undergraduate interviewee (Dinakar) shared his discomfort in the following way:

It's very different from what I have experienced back home in India... Yes. Writing assignments I think it was one of the biggest issue especially for an Arts student. In Humanities we are expected to write so many papers and mostly it is all internally assessed. With assignments we have never been formally taught on how to write, I mean structure properly and stuff in India. That was always a problem and it still continues to be I think. I might have ideas but I don't know how to put them into the right structure. I think initially I didn't get much help at University because I didn't know what they had in their mind when they just probably expected me to settle in with whole writing issue, but that didn't happen.

While the above experience illustrates the difficulty with academic writing, another interviewee Vineel, a postgraduate diploma student, mentioned his difficulty with oral communicative abilities. He described how his lack of fluency in spoken English affected his classroom participation. He put his experience like this:

Generally, I won't express my ideas as I feel that my English is not that good, as English is not my first language.

Despite these difficulties, the participants seemed appreciative of the value of the theoretical and practical aspects of their study. Their impressions about their teachers were positive as they did experience a different kind of student teacher relationship. Other than this, the teaching process allowed students to gain knowledge through practical aspects of learning unlike in 
India. This practice allowed them space to develop their thoughts and new ideas without being confined to text books. An illustration of this can be noticed in the words of a postgraduate interviewee Jasmine, who says:

In terms of teaching approaches and teachers, here teachers are more frank I believe, I could go up to them and ask as many things as I wanted and they were quite helpful, they go beyond the text-books. So that's where practical knowledge comes to place. In India they focus on text-books. So they just teach us what's there in the text-book and they expect us to cram and write the same thing in the papers. But here it is different. So they give us our space, so we are allowed to write and express whatever we want.

\section{Assessment procedures and 'Writing' issues:}

Some of the students found assessment procedures such as, writing assignments, completing assignments on time, working on group projects, giving oral presentations, and managing study workload, moderately or very difficult. Among these activities, a significant number of participants (36\%) indicated their difficulty with 'writing assignments' either in 'moderately difficult' or 'very difficult' categories (See Appendix 8).

Other than this, for an open-ended question in the survey requiring information on three most difficult things they have faced in adjusting to their academic work in New Zealand, 23 responses out of 106 specified either academic writing or writing assignments (See Appendix 5). Besides this, academic writing requires the observance of various conventions, for instance, critical thinking and analysis, developing an argument, referencing, avoiding plagiarism and paraphrasing. It will be interesting to note that while six students identified critical thinking as something they most enjoyed in New Zealand academic life, five found it most challenging.

During the interviews as well, three of them explained their individual experiences related to academic writing. A postgraduate student of Human Resources Management (Monish) shared his tale, that he never had an experience of writing essays during his studies in India and hence, he did not know the academic procedures involved in this concept. In his words: 
Initially I had to manage things, I had to put more hours into my studies, because it was completely new system to me. I was not aware of how to write essays and I have never done this kind of thing in India. ... what basically an argument is and how to develop your argument into your essay and how to critique it. Initially I had some issues.

For this student, the library staff of his university helped him by providing support to enhance his writing skills in the areas of how to critique and develop an argument in an essay.

... then I contacted many librarians... and they were quite helpful as well and they told me...how to write, how to search different resources and they showed me... some sample articles which pointed out how to develop an argument or critique an essay...initially, it was quite difficult.

This student further illustrates how acquiring knowledge on the academic requirements can be an incremental process, where the first step is to understand what an argument is, but then one has to learn what is required for getting higher grades.

...I was not happy with my scores initially because I scored all B or B+, in one, I scored C but yes when I understood the basic things now my score really improved, I'm now scoring $A$ or $A+$. So initially it was...bit struggling probably now when I'm aware of things how to do it then it's quite good...

Further, referring to another essential academic convention in the New Zealand education system, 'Referencing', he shared how it was a challenging issue for him and others:

Regarding the referencing, it was quite difficult for me initially because I was not aware of how to do it. In India we used to write bibliography, the name of the books that are used but here we have to follow very specific standard...it is quite good for us, actually if you are getting any ideas from someone else's sources, we should reference. Initially it was difficult, I took help again from my lecturers and teachers, and they told me how to improve it. They actually have online guides for referencing, how to do it, practical examples as well, which helped me a lot 
initially, but yes I'm still improving a lot in that but not 100\% correct, but yeah its good thing.

In a similar vein another postgraduate student in biological sciences (Vineel) commented on this aspect of referencing:

It's completely a change of patterns which I was not aware of. I did not know how to reference or cite the publications. Here they expect assignments to be critical where I used to make it just a report with all statistics and figures. Referencing with good standards is being expected here...

An undergraduate female student (Mahima) who was confused due to lack of knowledge and understanding related to these academic requirements specially associated with writing issues, such as plagiarism, referencing, and paraphrasing, highlighted the confusion experienced by her as well by a number of other students and shared her experience as below:

One more thing I would like to include is ...there is a lot of referencing in our essay writing and plagiarism is a huge issue here. Back in India we never bothered about this referencing in writing essays. In every course handbook there is a mention about plagiarism, but I was just confused what to mention and what not to mention. Paraphrasing is also another issue to be considered in referencing where I was a bit confused.

These experiences of the participants reflect the initial struggle of many new international students who have come from a different educational background. However, in the end they all seem to appreciate the value of learning the protocols of academic writing. The experiences of these students also suggest a need for the provision of appropriate strategies and support in the initial stages of their studies, to make them aware of these academic norms and to enhance their skills. 


\subsubsection{Strategies applied to cope with the academic issues}

The findings revealed mixed experiences where the participants enjoyed their academic life as well as faced challenges. Consequently, they were offered opportunities in the survey and the interview to share the strategies they adopted to deal with these difficulties. Out of the total of 105 responses received through the survey, 64 respondents revealed different strategies used by them, depending on their personal attributes and the social support they received. Some respondents reported multiple strategies in their responses. They also listed useful personal abilities. Broadly categorised they were: self-determination, personal attitude, approaching people for help, and previous exposure to work or education. Similarly, the main helping factors that were categorised under social support were: support from the lecturers, peers, friends and past students, support from the library staff, discussions with others, Internet, help provided by the academic clubs. A list of all these factors with frequency count is provided in Appendix 7.

The data from interviews included a number of strategies the interviewees applied to cope with the demands of their academic activities. Out of the total 15 interviewees, eight of them illustrated their strategies. These strategies included receiving help from their tutors, lecturers and professors, attending workshops on skills improvement, approaching library staff, spending long hours and putting extra efforts to accomplish their tasks. Among the interviewees, those with course work responded well in revealing their strategies to overcome academic barriers, compared to those engaged in thesis writing. Two participants of the interviews revealed that since they did not experience any academic difficulties they had not applied any specific strategies. Further, the common element identified in both of them was their English language proficiency which helped them to sort out their academic issues.

Victor: I haven't applied any specialised strategies. Once the language barrier is broken down it immediately breaks down all of the other barriers.

Jasmine: well, not really. I didn't face any problems as such, and one of the reasons could be because I worked with Infosys for two years, and my work was all concerned with international clients. So as I already worked with international clients for two years, I didn't face much problem initially. 
Two participants who resolved their academic concerns mentioned that they were able to get help from their tutors, lecturers and professors either through feedback or by seeking clarifications to deal with certain academic issues. One of them, a postgraduate student shared his strategy as below:

Vineel: Out of experiences with bad grades initially. I did have feedback from my professors and had many special meetings with them on how to improve my grades.

Two interviewees pursuing postgraduate studies revealed that they did spend longer hours to overcome these barriers and to accomplish the time bound academic requirements. One of them a postgraduate student (Suresh) in computer sciences illustrated his strategy which helped to overcome his academic challenges in the following way:

I've changed my routine accordingly. There wasn't really a strategy. I mean what was expected I started fulfilling that. If I was expected to be in the university from 9 in the morning to 5 in the evening, I was there. If I was expected to meet my professors, I met my professors. I did whatever was expected of a master's student and how a master's student was supposed to be in study. I have to spend much longer hours than anybody else sitting in the lab and working harder than the other people. There was only one specific strategy: to work hard and try to accommodate yourself into this system.

Likewise, an undergraduate interviewee explained that prioritisation of her academic tasks each week helped her to overcome the difficulties. One interviewee pointed out that by attending postgraduate workshops he was able to enhance his skills to tackle issues connected to writing and referencing. Another postgraduate student made use of the services of the library staff in receiving proper information or web links to improve his abilities and to overcome his academic barriers. One of the interviewees (Rajasekhar) who is nearly completing his doctoral study revealed that he actually unlearned 'a lot of things' as a strategy to overcome his academic challenges. His explanation of this concept is provided below: 
I have to actually 'unlearn' a lot of things which I learnt in India, such as what I have been taught, I have to just go backwards and I actually unlearn them. As I was strongly discouraged from my school 'not to question' or 'to follow the standard approaches' in dealing with certain problems in science. Obviously there is no unique solution to a problem and you can solve it in multiple methods. At least in academic life you have enough people around you who have lot of skills and they are very happy to share those skills with you. In my work place fortunately I have made friends who have such skills and who are likely to help me with these things. When I ask for any help they would be happy to help me or explain to me.

\subsubsection{Suggestions for betterment of conditions related to academic issues}

The survey indicated that some Indian international students, particularly the course work students, encountered difficulties in fulfilling their academic requirements, particularly related to the assessment procedures. When the interviewees were offered an opportunity to provide suggestions, based on their experiences, to improve the conditions, they came up with a few suggestions for betterment of conditions in their universities. Their suggestions included effective orientation programmes which should provide clear information on the availability of services and expectations of New Zealand educational system, support for improving academic writing skills, requirement of understanding the needs of international students from diverse cultural backgrounds, provision of centralised services to help international students, and continuous support with regular meetings and 'Buddy programmes'. Five interviewees insisted that it was the responsibility of international students to explore the conditions, to attend lectures regularly, to put extra efforts and to be flexible to accept changes of a new place.

Out of the 15 interviewees, seven participants requested effective orientation programmes. The perceptions of two postgraduate students are provided below:

Monish: Yeah, orientation is there but I was not much satisfied with the kind of orientation given to us. You can say it just like formal thing, that's it. They gave us information about university, about rules and regulation but it was overview sort of thing but not specifically for our courses. 
Suresh: I think they should run programmes for people coming from Asian cultures to tell them how different the system is from their previous system, what work ethic will be expected of them and what they need to do for the faster adjustment of themselves into the system. In the orientation they should tell them about all the services available and all the pros and cons of the system as compared to the previous system. For a masters or research student here you can't really go and ask your supervisors on the basic things and you have to do on your own, so they should be familiarised or told out. Then one can start adjusting and changing himself or adapting to this system.

Similarly, three other participants suggested that adequate information should be provided on the differences between educational systems in New Zealand. The opinion of an undergraduate student is as below:

Mahima: For international students when they come from a different academic system in orientation they should be given some introduction of this system, the plagiarism issue and other related matters. So students can mentally be prepared on what to do and that would be very much helpful.

Being involved in course work in his studies, Vineel, a postgraduate student, further suggested a remedy to avoid the worst consequences of dealing with an unfamiliar education system:

I believe that the university should let us know the pattern of assessment, assignments how they are made... They should... conduct some tutorials especially for international students during the orientation week. Other than this, some courses have ... 50\% of assignments and 50\% of examinations. If assignments are all submitted at once students end up making mistakes. Initially there should be an assignment for $10 \%$ or $5 \%$... This will help the students to know about the expectations of their teachers...

Six participants highlighted the need for guidance on improving their writing skills. While explaining the experiences with different educational practices of New Zealand, undergraduate 
students Dinakar and Mahima, and postgraduate students Vineel and Monish explained their woes with writing issues. Dinakar stressed the need for help and expressed his opinion in the following way:

I think it should almost be mandatory for the university to help international students initially with writing. I don't know how it is in other streams, but at least every Humanities student should definitely get some help with writing skills, developing writing skills and understanding the New Zealand way of writing styles. That is I think very important because everything here is being judged not on how much you know ... but on how well you write... The mode of assessment is also new to us and we've never experienced anything like this in India. Instead of trying to help someone after the assignment has been done and then trying to judge them on the lapses, if you can help someone at the beginning on the approaches, it would be better. They do that at tutorials where there is majority of local students and there may have been a couple of international students. But ... they do not have the same problems that we have.

Similarly, a doctoral student requested help as his academic requirement involves writing a thesis.

Prakash: I think in the initial period a formalised training should be given to all the new entrants because PhD is all about writing. It's all about producing a thesis. It's not a course work what you produce in your exam paper. So I think as far as my own Law faculty is concerned there should be a formalised legal training on legal writing in the initial days. There should be a bit training on how to do legal research, how to undertake and organise your stuff. Organising is a major task in PhD.

Three participants requested for 'Buddy' or mentoring programmes to help international students. Among them a postgraduate student shared as below:

Jasmine: From the university side one thing I guess could be a good option is to have a buddy system or mentor system. For example there can be mentors from the 
local students in New Zealand. For international students it is all new for us what we are going to learn and how it goes on. So if they have buddy system or mentorship for each and every international student it could help a lot.

Another doctoral student suggested the initiation of supervisors and other administrative staff to consider the cultural backgrounds of the international students in the following way:

Rajasekhar: The only thing I would suggest that there should be a little bit effort from the supervisor side and from the people who are in admin to understand the cultural backgrounds of the person they are dealing with. People don't open up as they have come from different backgrounds.

The responsibility of individual international students was also emphasised by a few participants.

An undergraduate student expressed his opinion as below:

Victor: Most of the academic issues come from difficulty in understanding the language and because of loneliness. To break that you should be more relaxed and be ready to absorb things from a different culture.

When the data related to this issue were coded, three broad categories of views were identified. Firstly, there is a need for effective orientation programmes with clear information on the differences in educational activities between India and New Zealand, and information on the availability of resources in their universities. Secondly, there should be a provision for improvement of required academic skills, continuous support from their supervisors/ lecturers/ tutors/ 'Buddy' programmes (mentors) to make them aware of these differences and to provide guidance to cope with their academic challenges. Thirdly, the responsibility of the international students to explore the conditions of a new place of study and putting individual efforts to adjust to the conditions. A detailed list of suggestions is provided in Appendix 9. 


\subsection{Experiences with facilities and support services}

Potential sources of support for international students include facilities and support services within their universities, and support from community organisations outside their universities. This section narrates the experiences and perceptions of participants in relation to the facilities and support services used by them in their universities. It attempts to assess the effectiveness of such facilities and services. In addition, social support offered by other community organisations are considered. The strategies applied by the participants to overcome their challenges will be presented. Finally, suggestions from the participants intended for both educational institutes and prospective international students will be listed.

The Figure 5.5 below demonstrates the levels of satisfaction of Indian international students with the overall quality of facilities and services in their universities. When the respondents of the survey were asked to rate the overall quality of these facilities and services, $84 \%$ of the total of 104 ranked them in the range of 'good' and 'excellent'. The satisfaction rate slightly varies between male (83\%) and female (88\%) respondents. However, it varied hugely between students living first time outside India (91\%) and others (41\%) who were not. Similarly, among postgraduate students with course work the satisfaction rate is relatively low (74\%) compared to the other categories of students where they had at least a minimum satisfaction rate of $83 \%$.

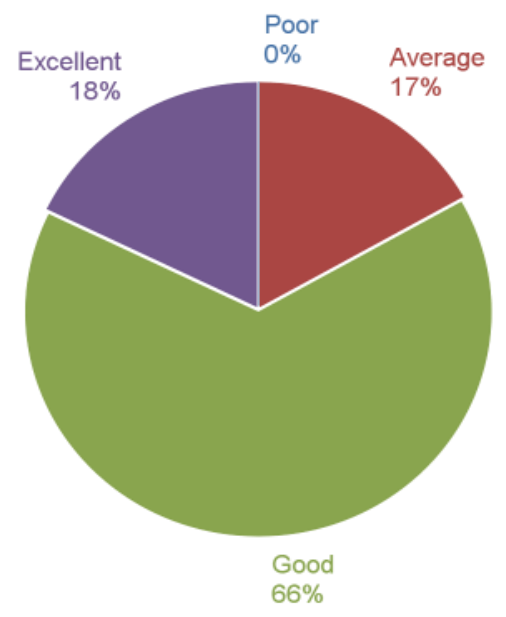

Figure 5.5 - Overall quality of facilities and services in universities 


\subsubsection{Experiences with services available in their universities}

To a question pertaining to the use of available facilities and support services, the responses indicated that most of the students were familiar with the availability of such services and used them. The collective responses ranged from 'moderately used' to 'very much used', (Figure 5.6) indicating that $98 \%$ of the Indian international students have used these facilities and services available in their universities.

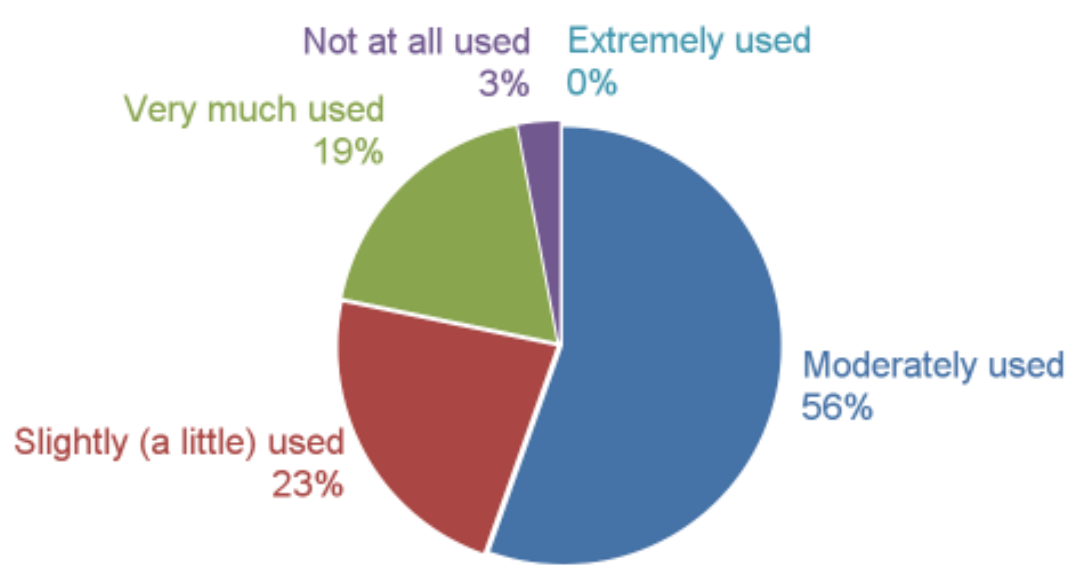

Figure 5.6 - Self-reported use of facilities and services

Again, out of 15 different types of services intended for them in their educational institutions, the students were asked to rate the quality of each service or facility. The list of services and the ratings given by the respondents of the survey to each of these services in the scale of 'poor', 'Average', 'Good', and 'Not available' can be viewed in the figure 5.7 below. 


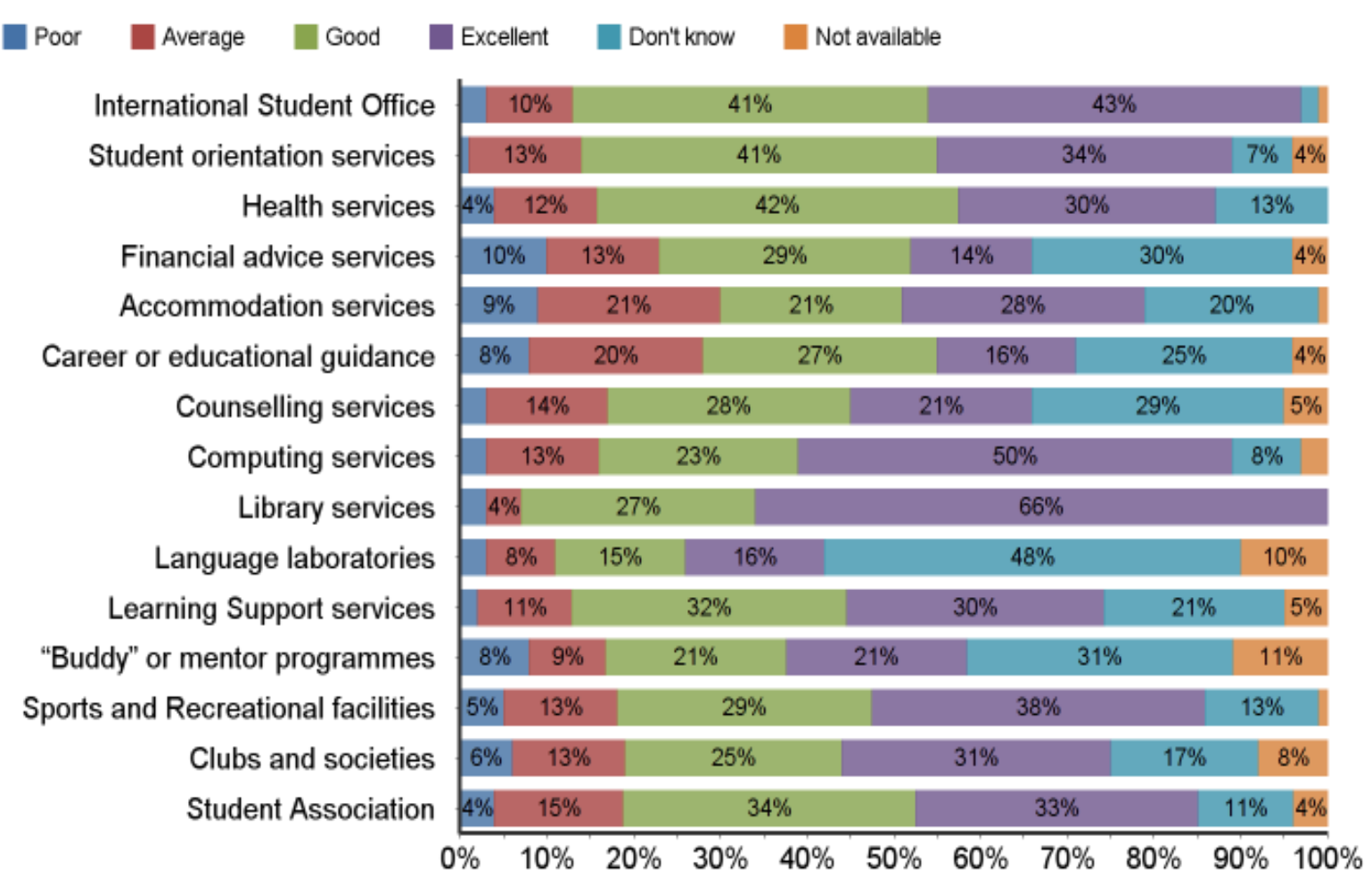

Figure 5.7 - Perceived quality of facilities and services

As rated by a total of 104 respondents the top three services and facilities with a combined rating of 'Good' and 'Excellent' were: library services (93\%), international student office $(84 \%)$, and student orientation services $(75 \%)$. On the other hand, the top three services and facilities in the combined rating of 'Poor', and 'Average' categories were: Accommodation service (30\%), Career and educational guidance (28\%), and financial advisory services (23\%).

It is possible that students who did not have a need for service were unaware of their existence in their institutions. Remarkably, $11 \%$ of the respondents indicated that 'Buddy' or mentor programmes were not available in their universities. Among this percentage of respondents, majority are male participants (82\%), doctoral students (64\%) and belong to four universities of New Zealand such as AUT, University of Otago, University of Waikato, and Victoria University of Wellington. Again, $10 \%$ of respondents who indicated non-availability of language laboratories were also majority male students (80\%), doctoral students (70\%), and studying at the same universities mentioned above. The study also exposed that a number of respondents 'did not know' about the availability of some of the services or facilities. Considering the percentage of respondents, the top four on the list under this category were: language laboratories (48\%), which, given the English language proficiency of the students 
was not surprising, "Buddy" or mentor programme (31\%), financial advice services (30\%) and counselling services (29\%). A detailed list of ratings of the respondents on the quality of each service is provided in Appendix 10.

Besides this, the respondents of the survey were asked to identify sources of support available to them in New Zealand for different personal and life challenges (Q.57). The trend of the respondents showed that they perceived other international student friends as their sources of support for many aspects, but with some exceptions. When troubled, upset, or lonely, $47 \%$ of these students preferred people from their home country as their sources of support. Similarly, $54 \%$ of respondents perceived academic staff as a source of support for academic issues. Except this academic aspect, the knowledge of respondents on the support available from their universities (for the other ten items indicated in the list) was limited and fluctuated between the percentages of 4 to 21 . Importantly, it was indicated by only four percent of respondents that support was available from their universities during their troubled times or whenever they felt loneliness. Likewise, Kiwi student friends were not identified as a source of support for most issues except for information about life in New Zealand. A detailed list of views of respondents about different sources of support in New Zealand can be noticed in Appendix 12.

It appears that there had been some variations in the views of respondents on their overall satisfaction on the quality and usage of services in their universities, and in their actual perceptions about each of the services. When the respondents were given an option to express their opinion if they were not satisfied with the services, eight respondents noted their comments. These comments mostly indicated their dissatisfaction related to the quality of support services in their universities, and a few of them are provided below:

R.20: There is no follow up on the queries. Neither is there any kind of follow up on International students and how they are doing or coping.

R.69: Do not expect student services to give a usual out of book reply to students' problems. If that's the case, [for the] student approaching them [it] is waste of time. 
R.106: ... I am not sure how far they are supportive in terms of crucial needs of international students. Especially when a student has difficulties with supervisors or any such kind of problems.

R.49: The University have got a poor career services where they give advice only on updating your $C V$ whereas the career service does not offer any graduate internship opportunity to student. In fact there are no placement or on campus recruitment opportunities.

Other than this, participants of the interviews shared their views on different support services and contributed detailed comments on them. The views of the interviewees provided a variety of impressions based on their personal experiences. Their views and perceptions on various services, such as orientation programmes, learning support services, Health services, International student service centre, Accommodation services, and career guidance services are discussed below.

\section{Availability of information through orientation programmes}

There were mixed opinions expressed by the participants of the interviews, having both positive and unpleasant experiences in connection with the orientation programmes. It appeared that those who attended orientation programmes had benefitted, and others who missed were adversely affected in their initial days of adjustment in New Zealand. One male interviewee (Victor) who has come to pursue his undergraduate course and attended the orientation programme in his university, said that he came to know about the support available to them through this programme.

During the orientation they did tell us about all the support ... we should get. They did give some booklets with information on what to do and where to go in case if we... were in any need of them.

Similarly, another undergraduate female interviewee mentioned that the orientation programme included different events and provided an opportunity to meet with other international students and Kiwi students. 
In a similar positive view a postgraduate female student (Jasmine) highlighted the importance of these programmes particularly to international students:

We had an orientation week before the commencement of classes and it was quite helpful because in the orientation week we were told ... what services ... were available to the international students, and it was particularly focusing on international students.

Another student (Pranith) who arrived late due to his visa problems and missed orientation programme, could finally attend a late orientation. He was offered a personal orientation by the university, however, he reckoned that his orientation was not elaborate enough to provide necessary information. Further, he mentioned that the person who presented the orientation programme appeared to be less interested in doing so and concluded it fast. He shared his view along with a suggestion like this:

I attended ... the last orientation... while the first orientation was for two hours and the last orientation was for 10 minutes. So you can just think how the information was compressed every next day. They gave me information about the things, but not in depth. But these things should be given at the pre-arrival stage.

Two other students from different universities who did not attend orientation programmes felt that as a result they were unaware of the facilities and services available to them. In some situations this lack of adequate information made them experience difficulties in the later days.

\section{Student learning support}

Three interviewees responded positively on the help they had received from the Student Learning Support Services, particularly in improving their writing skills. Four interviewees stated that they were not aware of the services available. Another four participants mentioned that they knew about the services such as Learning Support and Health counselling even before their arrival in New Zealand, but never used them. One student commented on the help from the student learning support though he had not used it. 
Prakash: Yes, we have Student Learning Support Services ... which is helpful to some extent. They are more useful for non-English speaking students. As my English is pretty $O K$... I didn't feel a major need for that.

\section{Health services}

Two interviewees indicated that they had used health services in their universities and one specified he did receive help with his stress related issues during the examination time. A female student narrated her understanding of health insurance provisions being an international student and explained how much it is helpful to them by describing the experience of her fellow international student.

\section{International Student Service Centre}

Two interviewees mentioned the assistance of International Student Service Centres of their universities regarding some visa issues. One other participant informed that he attended some social events and workshops conducted by support services centre. A female respondent at a different university explained the help she received related to accommodation arrangement, and the cultural and sports events conducted by the support centres. The following is an elaborate statement related to this matter:

Satya: From the university there are people who process our visa application and all those things. They also arrange accommodation for the international students. Before coming here we can't ask them for the accommodation. They also organize some cultural events for the new students where they can mingle up with the other international students. Time to time they also organize some sports activities, some family get together for all the international students. So they do help in settling the students over here. If someone needs a house, if someone needs to sell their goods, if someone is going and needs to organize or anything, we can just contact those people and they help us. Also they do send us the emails about the activities which are going around in (name of the place). If anything is going on, they will inform us. 
However, this feeling of appreciation was not universally shared, as another student (Dinakar) commented adversely on the events organised by the International Students Service Centre:

There was no help from the university to familiarise with the surroundings and with the local culture. Every now and then I would get emails (from the International Student Service Centre) which would say that there ...was an international student outing. But it was just like meeting other international students. I am going out and paying money and doing some activity, that sort of thing. That's not necessarily interaction with local people and not like one on one...basis. I don't think it's useful at all. I expect to have more of a cultural meeting than having these activities.

The opinion of the student relates to the general expectation of international students to develop contacts with domestic students and the response reflects some sort of disappointment.

\section{Accommodation services}

A majority of the Indian international students in this study noted that they did not opt for the accommodation arranged by their universities. While $80 \%(\mathrm{~N}=104)$ of the respondents opted for private accommodation arrangements, only $7 \%$ were utilising the accommodation facilities provided by their universities. Within this group (those accommodated in university halls), a majority (86\%) are postgraduate students other than the doctoral students, and the rest are undergraduate students. The other respondents (13\%) stated that they were living with their relatives or had home stay arrangements. When the students were asked to indicate the quality of accommodation services in their universities, around half of the students (49\%) stated that the services were either good or excellent, while 30\% rated the services as either poor or average. Interestingly, $20 \%$ of the respondents indicated that they did not know about this service in their universities. Additionally, when students were asked to comment on the accommodation facilities in the survey, a total of 46 responses were noted. The general trend indicated that the accommodation provided by their universities was expensive and 'flatting' (or sharing a flat) was a cheaper option. One of the respondent suggested a discount on the accommodation costs for students. 
R.67: It should be given on discount to students.

Another respondent commented on the services received from the concerned staff as below par:

R.20: I had to find my own accommodation. When I went to the student accommodation services they were not able to provide any help with finding any place.

Based on her own experience a female participant passed on her comments on the accommodation services of her university along with a suggestion like this:

Rashmitha: University does have an accommodation office and they should know at least who was coming, how that person ... has arranged his or her accommodation. If they provide the list of flats it is easy for the students to know the things. They should really make it accessible to the students. At least they should help, because accommodation is a very big thing.

Of the 15 interviewees nobody was found to be staying in the accommodation provided by their universities, and they all gave a standard answer that the university accommodation was too expensive and hence chose a private arrangement. Eight were accommodated with friends or flatmates. A few of the comments of the participants are provided below:

Victor: As an international student it is pretty much guaranteed accommodation facility but it is very expensive.

Monish: I'm sharing my room with seven guys over here and they are [from] different parts of the country. ... We are sharing accommodation as we can't afford University accommodation. It is quite expensive and around 220 dollars per week.

Another interviewee although recognised the reason for high cost, opted for private arrangement after his calculations as below: 
Pranith: There are some facilities for accommodation by university. But in my opinion it is a waste of money as it is very expensive. Minimum accommodation is $\$ 175.00$ and other expenses are $\$ 20.00$ or $\$ 30.00$, it ... goes up to around $\$ 210.00$.

Another Indian student found a few co-national friends who helped him initially and for that reason he continued to stay with them.

Vineel: I am staying with three of my friends who have come from our place. University does provide accommodation but I want to stay with my friends who initially supported me to adjust to the changes in New Zealand. I got good support from them. Our accommodation is quite comfortable.

\section{Career support services}

With regard to the career guidance services, $28 \%$ of the respondents rated the quality of the services provided by their universities as average or poor, and $43 \%$ rated them as good or excellent, $25 \%$ did not know, and 4\% responded they were unavailable (see Appendix 10, item 6). Among the interviewees, five of them stated that they were aware of the career support services in their universities, but did not utilise them. There were six doctoral student interviewees who stated that they were either on scholarship or did not have time to think about work opportunities. Three interviewees expressed their positive opinion on the services provided in their universities and one interviewee explained his adverse experience with the services provided.

Victor: Yes we do have Career Hub and Students Job search. I actually walked down to career services to get helpful suggestions on writing $C V$ and cover letter.

Vineel: There are such career services to develop our resume and to give some guidance on preparing for the interviews. 
Mahima: Yes. I went to career hub and student job search. After 2 months of my efforts I got 2 cleaning jobs and 'Student Job Search' is quite helpful in getting part time jobs. I work just 10 hours, but I am entitled to work for 20 hours.

One of the interviewee who had contrary opinion explained his experience as follows:

Dinakar: I've just started working and that is one more issue I've had with the university where they just do not provide enough support for international students to get part time jobs. There is something called Career Hub but I think it doesn't work especially for undergraduate students with the kind of jobs they have and they expect you to have experience. How would you have experience here and they don't even recognize if you worked back in India, they wouldn't accept that as an experience. So I think it's just a waste of time to visit Career Hub every now and then and trying to look for a job. Most of the Indian students that I have come across get some help through other Indian people in getting jobs. But since I didn't know any I had a lot of trouble in finding a job and that is a big financial burden. A part time job at least helps you to pay out rent and stuff.

The experiences of participants of this study demonstrated high level of satisfaction on the overall quality of facilities and services in their universities. Similarly, they revealed that almost all of them utilised the services in their institutions. The usage of facilities and services ranged between moderately used to very much used. However, when they were asked to rate the quality of individual services available in their universities, a small number of participants expressed their dissatisfaction with services like Accommodation and Career guidance services by rating them in the poor and average categories. Besides this, many participants did not perceive their universities as a major source of support except for academic issues.

\subsubsection{Experiences with the services available from other sources}

In the survey, the respondents were asked whether or not they had received any support from the Indian community groups. For this question, out of 103 respondents $69 \%$ stated that they did not obtain any help from Indian community groups. There were no other questions related to the support or services provided by other organisations outside their educational institutions. 
But in the second phase of data collection, the interviewees were given more flexibility for sharing their opinions related to the questions on this matter. This opportunity facilitated them to reveal more information on the other sources of support available from the community. Likewise, the participants also shared their experiences with 'educational agents' focusing on their significant role and influence in the enrolment process.

\section{Services available from the community organisations}

Generally, students reported that they were either not aware of any support provided by Indian organisations or that they had chosen not to use their services. Some of the views of the participants on support other than what they received from their educational providers are placed below.

Monish: There is a community which I heard of and I wrote an email, hoping to join that. But I didn't get any response from them.

Sunil: There are some Indian communities but as I don't have a private transportation facility, I didn't go.

Dinakar: I am not aware of any such community organisation and I haven't received any help and I just don't know about them.

Victor: No. I didn't see any support like that except for Maori and Pacific students.

Mahima: I am not aware of any organisation.

One positive response from one participant who had access to local support:

Satya: Yeah. I am connected to a local Indian community. They help in the sense we just have some family and cultural get together meetings, very limited not much supportive. 
One interviewee said that the local City Council had arranged a tour around places in the city, and afterward they were informed of the different services they could access if they needed them.

\begin{abstract}
Rashmitha: Yeah, there is some service from our city council. We were taken on a tour around the places in the city organised by the city council. There was a meeting in one centre and different people from the city council came over there told about the help in dealing with the legal things and some issues with the families, they have different services.
\end{abstract}

The experiences of Indian international students revealed that they did not receive considerable amount of support from other community organisations in New Zealand.

\title{
Services from the educational agents
}

The activities of educational agents were not covered in the survey nor in the interviews. But during individual interviews three postgraduate student participants on their own brought up the issue of agents and their operations in India. One of them stated that they were being provided with unreliable information:

Vineel: In India we were ... told by consultants that we are ineligible for scholarships, but Ifeel that there are some opportunities.

The opinion of the participant does not relate to any adjustment challenges during their stay in New Zealand, although it specifies a need that these students are to be provided with accurate information.

Another participant indicated the general trend of Indian students to contact educational agents while planning to study abroad. He opined that they were being provided with inadequate information. Further, he perceived the possibility of misleading students with dishonest information as the priorities of these agents were financial gains by increasing the number of enrolments in the concerned institutions. As a remedy, he made a suggestion for the intervention of the universities at pre-entry stage and shared his insights as follows: 
Pranith: Most of the students come through consultants (educational agents). The consultant doesn't give information to the students on how the course is being taught. Consultants only think about the commission they would get from the university. So university - students' relationship doesn't exist before the students come to the university. The university should contact the students before giving an offer letter. After coming here it's all over, and before coming only student coordinator should talk to the student ... that is necessary. For example: if you have IELTS with 6.5 you will get a seat here. But you need more than that here. ... When a student arrives he should be surprised with the situation here.

After coming here you have orientation. After coming here having travelled 12,000 kilometres, and after spending money if you tell like this you will go blank. Students should be given information before. ... a lot of communication gaps exist between the students and university. So ample information should be provided before arrival because this is the biggest step we take in our life and if something goes wrong we are dead.

So it was insisted that the responsibility of the universities was to offer adequate and reliable information on the realistic conditions of New Zealand. He felt that if reliable information was passed on to the students after their arrival, it would be too late and not be helpful. The perception of the participant was expected to help students to take informed decisions.

Another student (Suresh) who is currently pursuing his postgraduate studies in Computer Science shared his opinion indicating a different dimension of the problem:

Universities just need students and are very concerned with enrolling more number of students who are going to bring in international tuition fees. Educational agents are playing a vital role in persuading students to come here. The consultants don't really focus on education and they focus much on work afterwards. They would tell you what opportunities you have after studies rather than telling you about your studies. They won't tell you much about the university or courses and you have to 
find it on internet and websites. They try to attract people by telling them what opportunities they have in terms of work and settling down after education.

They tell that once you go abroad you will get a part-time job and you can support yourself. But that's not really the case and part time jobs are hard to come by and you have to struggle for months and months to get a job. So that is one false outlook what they portray.

While the above interviewee (Pranith) insisted on the responsibility of the universities, Suresh opined that prospective students should be careful about the services of educational agents. He advised students as below:

Suresh: The students shouldn't really trust all the agents in India or the picture they portray of New Zealand or any country. Because life is very different here from what was told to them. So life is very much difficult here. The agents in India really portray all sunshine ... of New Zealand. They exaggerate things.... They just want to sell.... But it is not really as nice as they tell.

These observations clearly indicate that there is a problem with at least some educational agents in India who are selling New Zealand education by giving students hope of work and settlement. But, nothing has been done so far by the New Zealand universities to control the activities of these educational agents.

\subsubsection{Challenges encountered by the Indian international students}

\section{Sad times}

In the survey, while reporting challenges of academic life, around ten percent of respondents indicated the experiences of isolation or loneliness during their initial adjustment period in New Zealand (See Appendix 5). At the same time, for a question about the sources of support available to them in New Zealand when they felt upset, troubled, lonely or homesick, only four percent of the participants considered their institutions to be their sources of support (Q. 57), 
(Appendix 12). A majority of the respondents mentioned other sources, such as people from their home country (47\%), other international students (23\%), home stay families (10\%), Kiwi friends $(3 \%)$, and others (13\%). It appears that many of the respondents were unaware of institutional support they could have access to if needed during troubled times.

Consequently, for those students who experience intense distress or significant problems, support may not be readily available, and the student may be too distressed to seek help. Dinakar, an undergraduate student shared his bitter experiences and the resulting consequences from his initial months in New Zealand, where he could not obtain timely support from his university:

I had a lot of issues ... I mean I went through a period of loneliness and depression and everything ... was very difficult to cope with. Since I ... was not receiving any help and I didn't know where to get help from. I ... had already started to put myself in a shell which started becoming more and more hard. Nobody would know until I failed, and there was one semester where I had failed in all my papers, because I was not attending the university. After I failed in my papers then the International Office realised that I ... had a problem and then they contacted me and they said about the available services and advised me on using those services.

This experience of the participant highlights the need for social support in relation to emotional aspects when students are living in a new environment away from their families and home country. It also highlights the need for staff to be aware of and alert for signs that international students are not coping. A provision of timely support from the relevant services may help the students not only to avoid psychological distress, but also prevent wastage of time and unnecessary financial burden. Once there was an awareness of Dinakar's situation he was able to obtain help from the appropriate services. Although the support received helped him in recovering from his challenging situation, he felt that it would have been better if he had received such help earlier.

... Then I started getting some help from counselling services which was very helpful. But still I mean it shouldn't be that an international student has to go 
through a period of suffering and then the help comes. It's more difficult to cope up on your own even though you are receiving help from student health and student counselling. You have already lost your semester which is a lot more money for international students than anyone here. There is a burden of all the money that you are paying and losing out of time and all those issues [Dinakar].

Dinakar emphasised the need for early intervention and precautionary measures from the appropriate support services of the university instead of offering support after noticing the students in distress. Similarly, another postgraduate student shared his similar feelings of loneliness while living in a new country and being away from his family:

Monish: Yes. You know sometimes you miss your country, so in these moments, you know, your local community or local help just to sit together. Sometimes you find it really hard to be in a different country and don't have anybody to discuss your problems or discuss your issues...

Relating to this aspect another interviewee proposed a viable solution which may help international students during these upsetting times. In his words:

Pranith: In the first days we miss family a lot and it takes time to get adapted. ... University should take the initiative by providing a coordinator for every ten international students preferably a person from their country who can interact with them to give some guidance. Because an Indian understand Indian better and a Chinese understand Chinese better. ... There are some students who return within one month. Why does he leave or why does he drop his course, it is because of depression.

Even though the other interview participants did not share any experiences related to homesickness or loneliness, the findings of the survey as well as the above interviewees highlight the need for a provision of timely support from reliable sources. Along with this, it is also essential to make the students aware of the available facilities to enable them to make use of these services whenever they require them in order to avoid adverse consequences. 


\section{Unawareness or obtaining information through unfamiliar channels}

This study shows that students may be affected by a lack of information that can be easily obtained from online sources. An interesting observation in this context is from a student who had never been overseas and at the time of interview had completed his first semester in New Zealand. He commented:

Pranith: In my first month I did spend $\$ 40.00$ to $\$ 50.00$ for taking print out. But later I have come to know that I was entitled to free printing as a master's student.

However, another participant who had been overseas prior to his arrival in New Zealand found a solution to his problem by accessing online sources. Possibly, unfamiliarity with the local conditions contributed to some students' difficulties. A couple of students reported that their friends alerted them to the available services. This issue is adequately reflected in the statements of interviewees quoted below:

Suresh: All the international students should be made aware of the services available to them and to make use of them. Most of the students I think are not aware of them. Here you will have everything, but they don't tell you about that. Only in the case of need when you ask for it or look for information on the website, you will come to know about that. In the orientation itself they should tell the international students about the available services.

Prakash: They should have a more robust and more systematised, specific approach to make sure that the students are aware of the availability of facilities.

Another postgraduate student found it in a hard way that it was not enough to have the information online, one had to explore it. He stated his experience in the following way:

Prakash: There are some financial supports... we can use up to an amount of $\$ 300.00$ per year for buying some research related books. We have a grant of $\$ 6000.00$ over a period of 3 years for attending conferences or for taking some 
research. But for this thing I did feel that there was no proper explanation on these things and everything you have to find out on your own by visiting the websites or digging into it. There was no one to explain beforehand about these facilities .... There was no formal guidance available.

From the above examples it may be inferred that while some students have benefited due to their ability and initiative to access information, others have suffered for not getting proper information either because of unawareness or due to unfamiliarity with the New Zealand practices. These experiences of the participants inform the need of every prospective international student to explore the conditions of the host country to make themselves familiar with different practices and to make use of the facilities and services effectively, to avoid unnecessary consequences.

\subsubsection{Suggestions for betterment of conditions of the support services}

Having realised the importance of orientation programmes, participants in their interviews provided some suggestions, particularly to suit the needs of Indian international students. For example, eight out of fifteen interviewees felt the need to be 'told' of the facilities available during orientation. In addition, they expect orientations to include, along with the information on the available facilities and services, information on the learning environment such as educational expectations and norms of New Zealand educational system. A suggestion by one Indian international student (Mahima) illustrates the need. According to her:

I would like to suggest giving students a good introduction in detail about the whole academic things because when we check online we just read terms or points. It should be in a person to person way to make students understand the things thoroughly. It should be done during the orientation week before the course starts which can avoid shock or surprise to the students.

One of the participants made a suggestion to the educational administrators to promote cultural festivals so as to improve the cross-cultural understanding of the international students as well as local community. A few students requested help towards accommodation arrangements and one of them proposed a discounted accommodation for international students. The role of 
educational agents was treated with much suspicion by the participants and required intervention of the universities to monitor their activities. Some other suggestions included necessary timely help from the universities to deal with their psychological issues such as loneliness or depression, particularly during their initial period of stay in New Zealand.

\subsection{Summary}

The first part of this chapter described the experiences and challenges of Indian international students related to their academic life in New Zealand. It also deals with their perceptions on New Zealand and its differences with India. Some students were baffled by differences between the educational systems of India and New Zealand. These differences mainly concerned the theoretical and practical aspects of the learning process, independent approaches to learning, designing their own degree by choosing courses, multi-method approaches to teaching and learning, and different kinds of assessment procedures. It seemed that students though valued these differences, felt the lack of proper guidance and information to deal with them. Further, the experiences of students revealed traces of racism among the academic staff and others, although the impacts were not so serious. However, most of them eventually coped well with the challenges and even enjoyed the academic environment.

The later part of this chapter presented the experiences and perceptions of Indian international students with respect to the facilities and support services in their universities or from other organisations in New Zealand. Overall, the Indian students were satisfied with the different services available. A few students who did not attend orientation programmes experienced some difficulties until they became familiar with different facilities and services in their universities. Some participants in this research revealed that they were unaware of important support services such as Student Learning Support Services. The accommodation facilities provided by the universities were perceived as expensive. Some participants expected more support in obtaining part-time work. A small number reported that the educational agents had misled them by not furnishing a realistic picture of conditions in New Zealand. The following chapter will discuss the experiences of the participants related to their social life in New Zealand. 


\section{Chapter 6 \\ Research findings: Social life experiences}

\subsection{Introduction}

This chapter presents the findings on Indian international students' experiences of their social life in New Zealand. For the purpose of detailed analysis, these experiences are further divided into two categories, i.e., personal and social experiences. Along with their experiences, their perceptions of different cultural aspects and challenges encountered in New Zealand will also be discussed. Following this, a note on the level of their satisfaction with life in New Zealand will be presented. Next to this, a few suggestions for the improvement of living conditions proposed by these students will be presented. Finally, this chapter will conclude with a brief summary of the findings of this chapter.

\subsection{Experiences of personal life in New Zealand}

The personal experiences discussed in this section relate mainly to their living arrangements, part-time work opportunities, and leisure time activities.

\subsubsection{Living arrangements}

Most of the respondents to the survey were living in private rental accommodation $(80 \%$, $\mathrm{N}=104)$ ). Thirteen percent of the students were either in home stay arrangements or living with relatives. Only a small percentage (7\%) were living in student hostels/halls of residence. Additional comments $(\mathrm{N}=46)$ on accommodation facilities in the survey revealed that many students felt that university accommodation was very expensive and that flatting (or sharing a rented flat with a few other students) was a cheaper option.

Those interviewed also confirmed the situation. None of the 15 interviewees lived in university accommodation. Five interviewees indicated that they had their own private living 
arrangements. Four interviewees specified that they preferred living with other Indian friends. Another four interviewees informed that they were flatting with others. One female interviewee was having home stay arrangement, and another interviewee was living with her partner. One of the female interviewee commented that accommodation provided by the university involved six months' down payment which was not affordable to her, and as a reason opted for private arrangement. She commented:

Mahima: I was looking for help to get my accommodation, but it was not very helpful as it didn't fit into my budget. I was asked to pay for 6 months down payment and it was quite a problem for me. I am not really very happy with the accommodation services of [university name]. I do expect more. When new international students are coming, there should be an opportunity to have some temporary accommodation for a couple of months to figure out which should be a better option for them.

Another postgraduate student also made a similar request seeking universities to provide free accommodation for a week and allow them to make a choice on their accommodation:

Monish: One suggestion I would like to say, you know, when international students come, university should provide at least a week of accommodation free of cost so that you get time to find your own house, if you are not staying on the campus.

Unfortunately, since none of the interviewees lived in university accommodation, it was not possible to find out the experiences and advantages the students might have by living in the university halls. However, the interviewee who had home stay arrangement, revealed that she had benefitted in two ways, such as discussing her issues with a Kiwi lady at home and improving her language abilities through long conversations.

\subsubsection{Experiences and challenges with part-time work opportunities}

When the students were asked if their student permit allowed them to work part time while studying in New Zealand, most of them (97\%), out of a total of 103 respondents, stated that 148 
they were eligible to work. A few other students (3\%) noted that they did not know whether or not their visa allowed them to work (Figure 6.1).

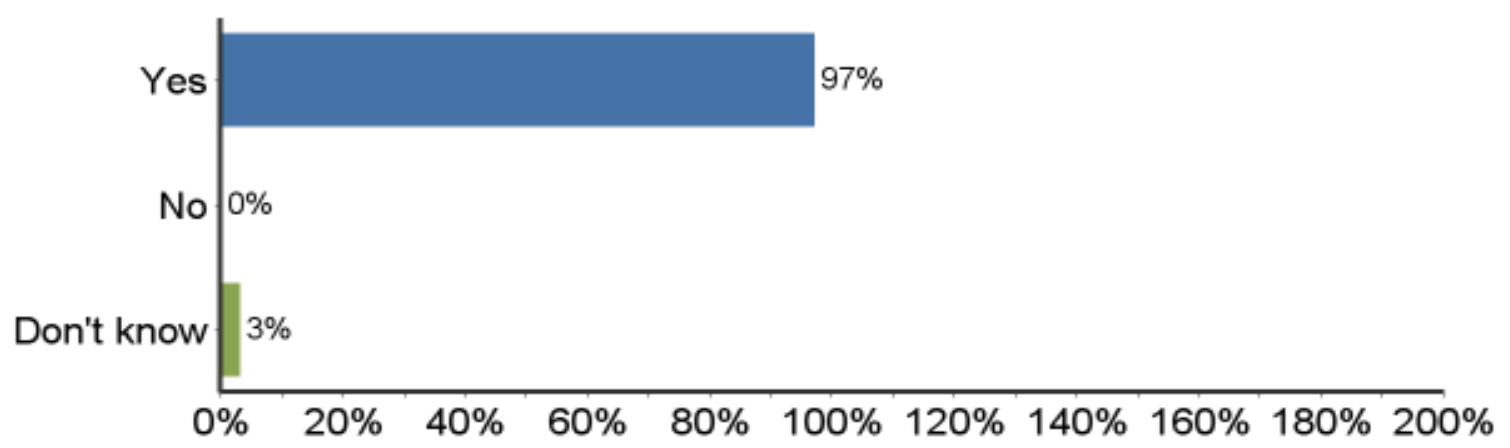

Figure 6.1 - Percentage of students eligible to work part-time

Since students were eligible to work part-time in New Zealand, they were further asked whether they had tried to find part time work while studying. About $41 \%$ (Figure 6.2) said that they were currently working and this was followed by $25 \%$ of students who had not succeeded in finding any job. The remaining $34 \%$ of the respondents stated that they were not working for other reasons. It is notable that the percentage of students currently working is less than half of the eligible students.

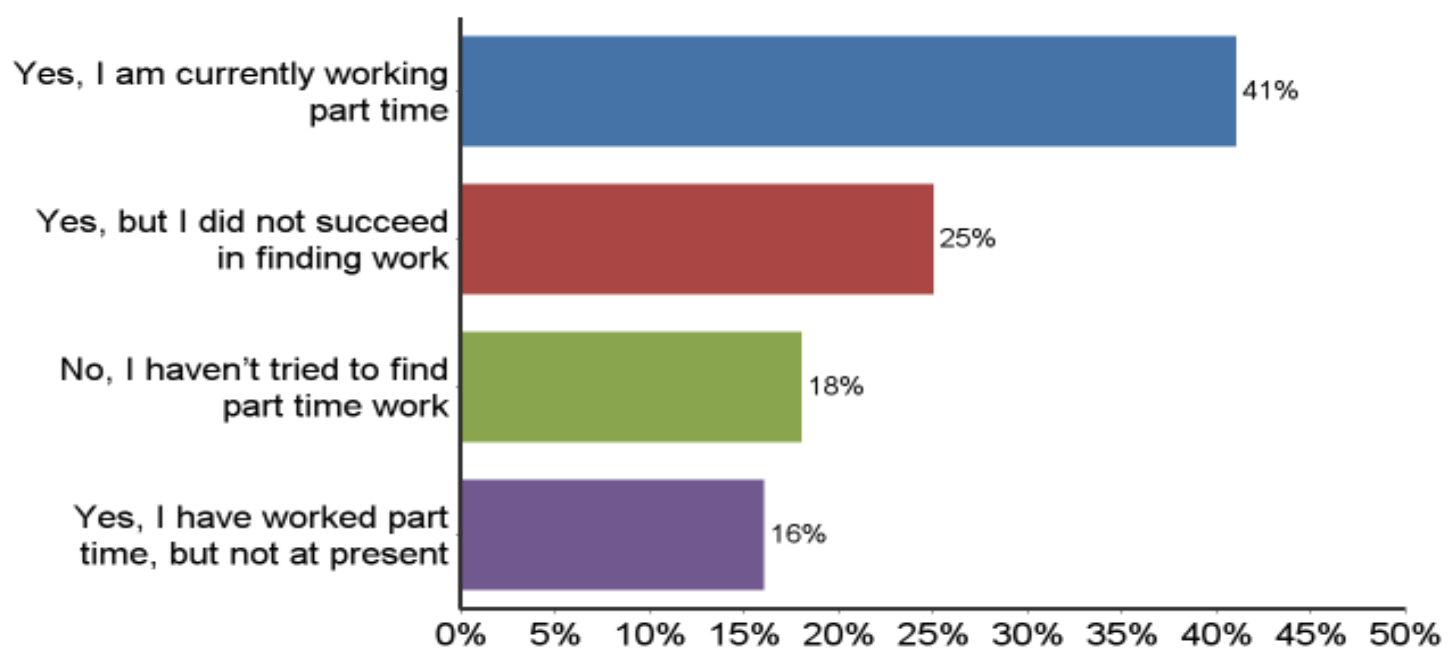

Figure 6.2 - Percentage of details related to part time employment 
When the respondents were asked whether they faced any difficulty in gaining part-time work, a majority of them (73\%) (N=98) stated they had. If they faced any difficulty, it was asked through an open-ended question to elaborate their experiences. In response to this question, 54 responses were noted by the respondents. In brief, the responses indicated a few important factors which are provided below:

Two respondents indicated that they could not succeed because of their international student status.

R.48: To get a good job is difficult as an International student. SJS and [name of the university] career hub are useful, but not too bright in finding a job ...

R.45: At some places the employers prefer more than international students, as per my personal experience. Also, many times people don't like to give chance to a fresher, but unless freshers get an opportunity, they will never gain an experience.

Two respondents believed that their Indian name affected the chances of achieving a part-time work. They further perceived that employers seemed to have a fixed notion that international students had inadequate levels of English language abilities.

R.2: There was apparently a general impression of Indian students [with no prior NZ work experience] not being good communicators or not being a good cultural fit. This would result in early rejection since many companies are not willing to take that risk.

R.83: Felt discriminated against for two years, which I attribute to my name, because employers take one look at the $C V$; as soon as they see the name or that the applicant is an international student, they assume that our English is incomprehensible and that we are incompetent. However, I feel that my English is a lot more fluent and easily understood than that spoken by most residents of this country. In this regard I'm deeply upset that it has taken me two years to find a job with the same $C V$. 
A few responses stated the limited part-time work opportunities in New Zealand.

R.63: Applied to many organisation, got rejected many times.

R.54: It is extremely difficult to find a job in New Zealand.

R.52: I have been here for 1.3 years and I got a part time only this year after I tried hard to get a job.

Employers require local experience and references:

R.62: Requires NZ experience even for part time. As an international student how can I have that?

R.67: Demand of reference and experience in NZ, which is difficult for a new student.

R.73: I have visited at least 20 places for job, everyone here need reference. How could a person coming to NZ first time in his life give reference!!!

In the individual interviews too participants came up with both positive and negative experiences with regard to part-time work. Some of the interviewees who had succeeded in obtaining work narrated their experiences as below:

One of his friends helped Victor to attain a part-time work opportunity.

I work in lunch time hours in a café.... one of my friends is working there and when they needed more people I got an opportunity through her.... 
Another interviewee explored the opportunities through a newspaper and succeeded:

Monish: Yeah I work part-time. I actually was going through a daily newspaper. I found out that there was a new Indian shop which opened in (Name of the place). So I went to that shop...I'm looking for a job, so the guy was very nice and he ... called me after one week. So that's the way things worked out.

Another postgraduate student distributed his resume at different locations in the city on his friend's suggestion and achieved his goal:

Suresh: I work part time in weekends...I just went around the city handing out my resumes and one day I got a call.... One of my friends suggested me to go out and to give my resumes in different locations ... It is very hectic I would say as managing work with my studies. My degree is very demanding but at the time being a graduate student and being 25 years old, I just don't feel like asking my parents for money. So I like to work and provide for myself. But the schedule is very nice, Monday to Friday I study and Saturday and Sunday I work. It's all balanced.

One female interviewee shared that she works now and then:

Rashmitha: Some casual work, not every time but sometimes only....Work experience is pretty challenging.

She further explained one of her observations with the university staff associated with this matter as below:

Rashmitha: Student job search is our main site where we find our jobs. There was a lady there I don't know what was the problem with her. Every time any international student went there without listening to their experience, she says that their language is not good and they can't communicate properly. She says that she can't give that job, and many students had this experience with her. Obviously, she is very biased. 
Equally, a few of the participants of the interviews also expressed their challenging experiences related to this point. One of the interviewees believed that his Indian name was one of the reasons for not succeeding with job hunting and shared his feelings in the following way:

\begin{abstract}
Monish: Initially for three months I was struggling to find work here. I was totally dependent on SJS website and I literally applied for say around 50 or 60 jobs and didn't get a single response from them. Now I understand that may be because of us being an Indian or being an Asian they didn't worry about it.
\end{abstract}

The perception of discrimination extended into the employment context, with one participant who worked part-time, describing negative experiences at work, which he believed were because of his ethnicity.

Vineel: Basically we do have good and bad things with customers. Sometimes they are very rude to me, but I feel it is all because of my ethnicity... When a number of people are lined up to pay their amounts, some impatient customers shout at us ... where we feel very depressed... Some people are really good and greet me. They are nice to me.

\title{
6.2.3 Leisure time activities
}

In the survey the participants were asked to provide information about the activities they were involved in during their leisure time. Out of a total of 103 responses the reported daily or weekly activities have been listed below according to frequency ratings. A few significant activities in that order were:

- spending time on online social networking such as facebook/twitter (81),

- $\quad$ eating Indian food (77),

- $\quad$ listening to Indian music (73),

- talking to friends and family members in their home country through internet resources (74), and

- watching television (64). 
The responses indicated that three quarters of the respondents were using social networking sites on daily basis. A similar number of respondents reported being in touch with friends and families through Skype either weekly or on daily basis. It appears that these students have been utilising the social networking facilities to acquire emotional or advisory support from their friends and family members in India or from other places in the world. At least half of the respondents were experiencing Indian music and food every day. The data suggest that during their stay in New Zealand they are mostly associated with social media activities, maintaining their relationships with their friends and families in India, listening to Indian music, and watching movies. A full list of leisure time activities of the participants from the survey is provided in Appendix 11 in accordance with their order of preference.

In this context, the survey included an open-ended question seeking to know any changes in their leisure activities after arriving in New Zealand. For this, 70 respondents reported with all 93 responses. Among these, 74 responses $(80 \%)$ specified change of leisure time activities, 17 responses indicated no change of activities, while the other two were unsure about the changes. The patterns of responses indicated interesting changes in their leisure time activities. A good number of respondents were utilising leisure time to accomplish their academic tasks. While some respondents' activities changed to in-door activities, a few others became attracted to out-door activities. While some respondents found more time to spend with friends compared to what they did in India; for some, social life was reduced significantly. A good number of responses specified better changes in their life styles and health aspects. The detailed responses showing the changes in their activities are shown below in table 6.1.

Table 6.1 - Responses showing change of leisure time activities in New Zealand

\begin{tabular}{|c|l|c|}
\hline S.No. & \multicolumn{1}{|c|}{ Change of activities or habits } & \multicolumn{1}{|c|}{\begin{tabular}{c}
\multicolumn{1}{|c|}{ Frequency of } \\
responses \\
$(\mathbf{N}=74)$
\end{tabular}} \\
\hline 1 & $\begin{array}{l}\text { Spending leisure time for academic activities } \\
\text { (time management/ academic work) }\end{array}$ & 15 \\
\hline 2 & $\begin{array}{l}\text { In-door activities } \\
\text { (More social media (5),watching movies (6), watching } \\
\text { movies reduced (1), listening to music (1), with family } \\
(2), \text { cooking (3), at home (4)) }\end{array}$ & 22 \\
\hline 3 & $\begin{array}{l}\text { Out-door activities } \\
(\text { Sports (6), Became adventurous (3), outdoor activities } \\
(5))\end{array}$ & 14 \\
\hline
\end{tabular}




\begin{tabular}{|c|l|c|}
\hline 4 & Spending time with friends (6), social life reduced (5) & 11 \\
\hline 5 & $\begin{array}{l}\text { Changes in life styles } \\
(\text { Life style changed (5),spending time for fitness (1), } \\
\text { more disciplined (1), health conscious (1), food habits } \\
\text { changed (2), more spiritual (1), fitness activities } \\
\text { reduced (1)) }\end{array}$ & 12 \\
\hline
\end{tabular}

A few of them stated that shortage of money, finding less time, and having a limited social circle were also valid reasons for changes in their activities. Some respondents believed that the changes they had experienced were good, but a few others were not happy with them. A few of those views are provided below.

\section{Some positive views:}

R.14: A lot! I do tramping and traveling which I hardly did in India. The discussions with friends have changed to philosophy, psychology, world politics, history and science, whereas I was never interested in such stuff in India.

R.24: After arriving in New Zealand my daily routine has improved tremendously, I have developed good eating habits as well as healthy leisure activities. I am following a good time-table and taking time out for everything including my studies.

R.31: Changed a lot. Made me to think more about the value of leisure activities, particularly with regard to fitness and maintaining a healthy lifestyle. Also, the art of managing work, study and leisure activities was learnt after my arrival in New Zealand.

R.108: Quite a bit. I can do a lot more of the outdoor activities that I was interested in but could not do often in India - sports, trekking, etc. I am able to travel or visit places in NZ more easily and with less time wasted on planning. 
The changes mentioned above in the lifestyle of these students imply that due to exposure to a different culture, there has been a tremendous transformation in their thinking about their habits. Moreover, they could find some facilities or opportunities to pursue their personal interests.

\section{Some negative views:}

R.49: I used to spend a lot of time with my friends back in India, but ever since I have arrived in New Zealand I am not able to make any local friends, so I remain at home, socialize through facebook and surf the internet.

R.53: Quality of social life has deteriorated drastically since I have been here. It's not easy when most people expect you to act a certain way because of the stereotypes.

R.85: Since I don't own a conveyance it has restricted me going out frequently, so I either spend my time at my flat on my laptop or probably go to the library in the university or visit my friend nearby.

R.4: There are many things which we can't do due to the shortage of money. Spending time at home is the best way to save money, but sometimes it really becomes frustrating and lonely.

Despite a few respondents expressing their negative views towards their leisure time activities, a majority of them were found to be spending their time positively with the activities they perceived to be better and interesting to them.

On the other hand, , the responses from the 15 interviewees reveal that many of these students keep themselves engaged in various kinds of social and cultural activities. In order of frequency their preferences were for: watching TV or movies, online chatting with friends and family, reading books, accessing social network websites, going shopping, cooking, going to gym, and visiting beaches. And it is here that it is difficult to discern any difference between them and any other student group in the country. 
The students were also asked if they were involved in any sports and recreational activities. The majority of the interviewees said that they were involved in some kind of sporting activity, such as football, basketball, cricket, badminton, taekwondo, cycling, gym, and tramping. However, some interviewees said that they were either not interested in playing or did not play at all. There were various reasons given for not playing, such as previous injury, lack of time, lack of transport, lack of interest, lack of other players, and not interested in joining a club.

\subsection{Experiences of social and cultural life}

These experiences are related to different activities of Indian international students in their social life in New Zealand. These are mainly related to their engagement with Indian culture, perceptions of cultural inclusiveness and differences, experiences or perceptions of discrimination, opportunities for friendship with local students, and opportunities for religious practices.

\subsubsection{Opportunities for maintaining Indian culture}

In order to explore whether or not the Indian students were having opportunities to experience aspects of Indian culture such as food, news, music, and movies, a few questions were set in the survey, and the respondents were asked to specify their usage in terms of frequency. The 103 responses received indicated that for certain items mentioned above they were frequently using them on weekly and daily basis: Indian food (75\%), music (71\%), news (52\%) and movies (43\%). Nonetheless, there were lower weekly and daily frequency of reading Indian magazines (17\%) and of participation in Indian community activities (19\%). These results indicate that there are opportunities available for students to maintain their Indian cultural aspects even in New Zealand with less or more frequency. The details of other frequencies are also presented in figure 6.3 below. Further details on the responses along with other items can be viewed in Appendix 11. 


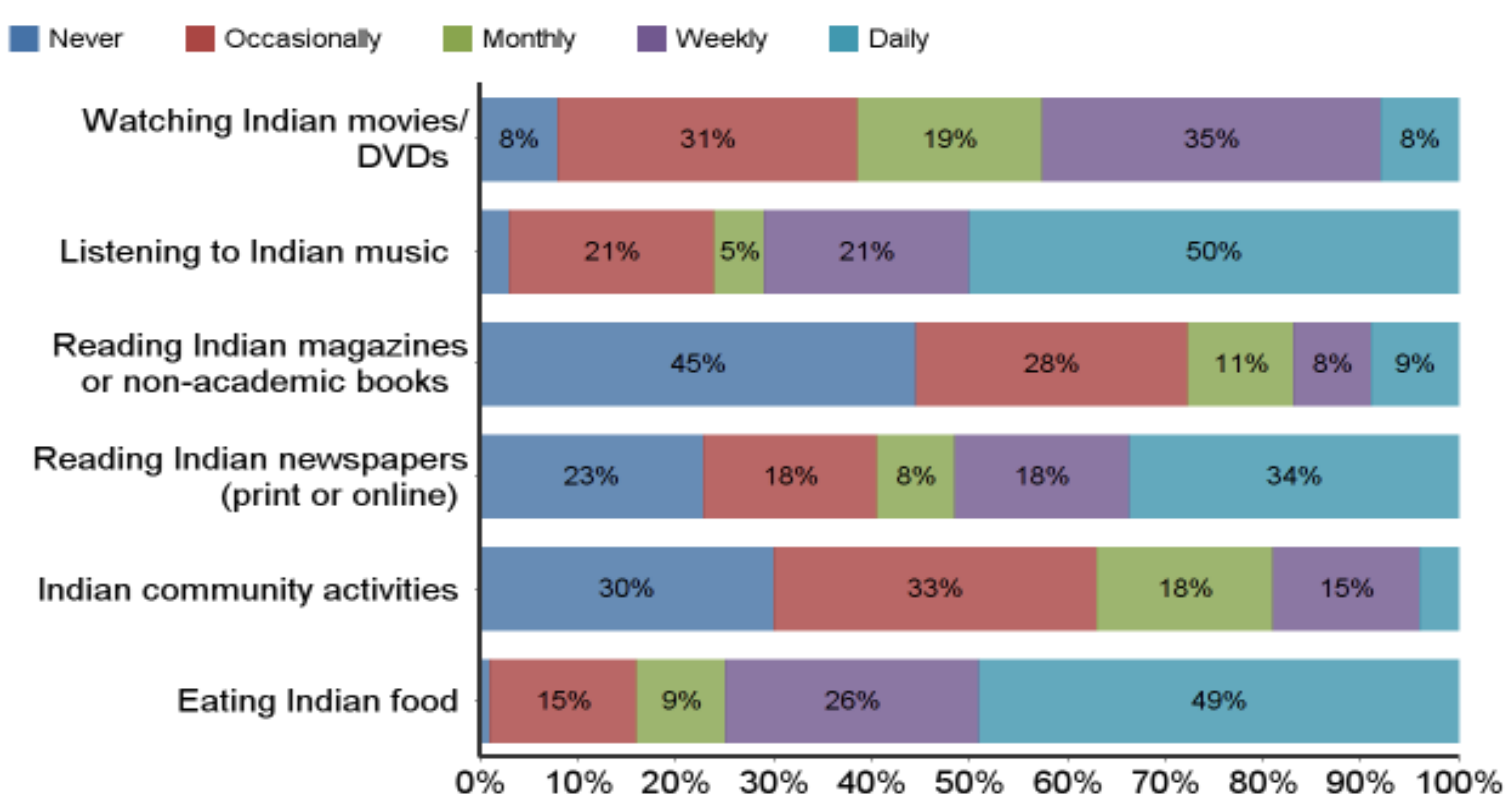

Figure 6.3 - Percentages showing accessibility to different items of Indian culture

Similarly, the interviewees were also asked to indicate whether or not they were having opportunities to experience Indian culture in New Zealand. Almost all of them noted that they were utilising the opportunities available to them. Indian music and Indian movies are available, and so are plenty of restaurants with Indian food. Likewise, there are shops which provide groceries required for cooking Indian dishes. One of the interviewee positively stated as below:

Pranith: Yes, we are getting Indian movies every month in [name of the city] and music with a click upon my computer. Food is expensive if you go out and eat. If you know cooking, you get every item here. We don't miss any of them here.

However, one student's experience is worth noting in this context, as it provides insights into the initial difficulties of an Indian international student who is a vegetarian. He shares his experience as below:

Monish: Yeah, I cook my own meal....Initially, I did not. For the first month I was dependent on canned food because I'm a vegetarian and so I don't eat meat. It was quite difficult for me to look out for vegetarian food. So I was dependent on the baked beans, canned food or maggi [noodles] for the kind ...because I brought 
some packets from India. So initially one month I was ok with that and with breads.

But now, I'm aware of the shops where I can buy Indian stuff, so it's now ... fine.

The experience of this student emphasises once again the importance of proper pre-arrival information to minimise their challenges in social life.

\subsubsection{Perceptions on cultural inclusiveness and differences}

In the survey a few questions were set to extract the views of the participants on their experiences of inclusiveness in a different culture. A majority of the participants (80\%) of the total of 101 felt that the cultural differences were respected in their institutions and most of them experienced cultural inclusiveness in their classes. Furthermore, $71 \%$ of the participants agreed that their lecturers/supervisors understood their problems as well as cultural differences in their learning styles. At the same time, many of the participants felt that there was comparatively less initiative on the part of their lecturers/supervisors in making special efforts to help international students $(61 \%)$, and in encouraging contacts with local students $(55 \%)$. The following table 6.2 provides detailed percentages of each category related to this matter.

Table 6.2 - Cultural inclusiveness of the participants in their institutions

\begin{tabular}{|c|l|c|c|c|c|c|}
\hline S.No. & \multicolumn{1}{|c|}{ Question } & $\begin{array}{c}\text { Strongly } \\
\text { disagree }\end{array}$ & $\begin{array}{c}\text { Mildly } \\
\text { disagree }\end{array}$ & Neutral & $\begin{array}{c}\text { Mildly } \\
\text { agree }\end{array}$ & $\begin{array}{c}\text { Strongly } \\
\text { agree }\end{array}$ \\
\hline 1 & $\begin{array}{l}\text { My lecturers/supervisors } \\
\text { encourage contact between } \\
\text { international and local } \\
\text { students }\end{array}$ & $13 \%$ & $7 \%$ & $24 \%$ & $13 \%$ & $42 \%$ \\
\hline 2 & $\begin{array}{l}\text { My lecturers/supervisors } \\
\text { make special efforts to } \\
\text { help international students }\end{array}$ & $11 \%$ & $5 \%$ & $24 \%$ & $29 \%$ & $32 \%$ \\
\hline 3 & $\begin{array}{l}\text { Cultural differences are } \\
\text { respected in my institution }\end{array}$ & $4 \%$ & $2 \%$ & $14 \%$ & $27 \%$ & $53 \%$ \\
\hline 4 & $\begin{array}{l}\text { My lecturers/supervisors } \\
\text { understand the problems } \\
\text { of international students }\end{array}$ & $9 \%$ & $9 \%$ & $11 \%$ & $35 \%$ & $36 \%$ \\
\hline
\end{tabular}




\begin{tabular}{|c|c|c|c|c|c|c|}
\hline 5 & $\begin{array}{l}\text { In my classes there is the } \\
\text { opportunity for other } \\
\text { students to learn about my } \\
\text { culture }\end{array}$ & $19 \%$ & $12 \%$ & $25 \%$ & $25 \%$ & $20 \%$ \\
\hline 6 & I feel included in my class & $6 \%$ & $1 \%$ & $16 \%$ & $29 \%$ & $48 \%$ \\
\hline 7 & $\begin{array}{l}\text { My lecturers/supervisors } \\
\text { understand cultural } \\
\text { differences in learning } \\
\text { styles }\end{array}$ & $8 \%$ & $9 \%$ & $15 \%$ & $31 \%$ & $37 \%$ \\
\hline 8 & $\begin{array}{l}\text { My classmates are } \\
\text { accepting of cultural } \\
\text { differences }\end{array}$ & $4 \%$ & $6 \%$ & $20 \%$ & $29 \%$ & $41 \%$ \\
\hline 9 & $\begin{array}{l}\text { Students from different } \\
\text { cultural groups work well } \\
\text { with each other in my } \\
\text { classes }\end{array}$ & $5 \%$ & $6 \%$ & $16 \%$ & $30 \%$ & $44 \%$ \\
\hline
\end{tabular}

Since Indian international students are exposed to the different culture of the host country, the participants of the interviews were asked whether or not they could identify any aspect of cultural difference between India and New Zealand. The responses indicated that they were acutely aware of cultural variances in many areas of social life. The variances are related to different attitudes to life, party culture, punctuality, holiday spirit, independent nature, freedom given to children, respecting everyone equally, open society, food and dress habits. A few related observations in this context are given below.

A newly arrived participant of the interviews shared her feelings on the living styles of the New Zealanders as below:

Razia: Here people take life easy. They don't really complain about things. They take life as it comes, they make it easy for them....People really don't cook here a lot at home and they live on outside food. I see ... early morning people walking into restaurants. That's new to me. I don't say that doesn't happen in India. There are bachelors and others, but here even if it's a family they still go to a restaurant. So they don't cook much at home here. 
Another interviewee highlighted the party culture in the local society in the following way:

Kishore: For people here weekend starts from Thursday. Students and ... many people, may be few people, start the weekend from Thursday. In weekends people shout during nights at 1 or 2 O'clock ... I'll be awakened at times because I stay next to the bar. Probably drinking culture is more. It is also there in India, but I think it is more here. On formal occasions we don't drink in India, we don't have that culture...I don't say it's bad, but it is a culture here...That was about the weekend.

A female participant compared the upbringing of children in India and New Zealand:

Jasmine: Here children are quite independent and they don't consult their parents for each and every thing, like we do in India. It's just the upbringing differences I believe. It's just the culture that you are brought up in...

A male interviewee also observed this view point and shared his evaluating opinion as follows:

...Here it's more independent, whereas in India it is more dependent on each other, such as depending on father or mother. There is no spoon feeding here and every individual should be on their own. That is the good thing about this culture, but there are some negative things in that. When you are not guided or taken care of, you may go wrong and when you are too independent you may go worse.

Having identified a good number of cultural variances and different aspects of lifestyles of local people, the participants of the interviews seem to have made their individual assessments by comparing them with their own culture. At the same time, the interviewees were asked to state if they were impressed with any aspects of the lifestyles of the local people. The usual response was that they were impressed with some particular aspects, such as: passion for sports, helping nature, the habit of greeting one another, listening to people, valuing opinions of others, 
punctuality, honesty, adventurous nature, organisation, making life easier by aptly using technological resources, and provisions and facilities for senior citizens.

One undergraduate interviewee stated that she was highly impressed with the Kiwi work culture and shared her observation as below:

Mahima: Here in New Zealand, when people are working they are working, and when they are relaxing they just really relax, that is what I like. Both things don't get mixed up and you can take the maximum benefit ... that is what I like about Kiwi culture.

Another participant was much impressed with the care towards the older people in New Zealand and disclosed her observations as hereunder:

Jasmine: Here I find people who are old ... have good facilities that are not there in India. For example, old people have free bus tickets here and in India it's not like that. They are quite respected and they are taken care of and I am not sure about what all services are available for old people, but they are quite independent. In India that's more of dependency for older people. Here older people who are 90 plus seem to be fit and fine and they are quite happy, happy in the sense that they have so many facilities available from the government.

A doctoral student shared his observation distinguishing the impacts of parents and society on the individuals in the following way:

Rajasekhar: In India as a kid growing up there, certainly there are a lot of pressures to fulfil certain aspirations. Parents here also want you to be successful, but I don't think they drop [impose] their values or opinions on to you. There is society around you, judges you all the time back home in India and you are too scared. Somewhere down the line you carry that and I carried ... that to here. Because of that all the time I am scared of opening up and I am always afraid of being judged. People ... back home are really interested in how you conduct yourself. Here they aren't, and it's up to you to lead your life and I really appreciate that aspect of New Zealand culture and lot of western society. 
In explaining their impressions, interviewees compared local conditions with that existed in India in matters related to working conditions, treatment of older people, and judging nature of the society around them. Although we cannot consider these impressions as a general voice of Indian international students, but Rajasekhar's view point explains one of the reasons for international students not opening up; it is not always because of a lack of language ability.

\subsubsection{Experiences or perceptions of discrimination}

The survey provided an opportunity to the participants to reveal if they had faced any unfair treatment from other students, staff or wider community because of their nationality or cultural background. The responses showed that overwhelming percentages of students 'never' or 'seldom' experienced any discrimination in their academic institutions or social life in New Zealand (see Figure 6.3. below). However, although discrimination was not identified as a major problem, there were some disturbing revelations as well. The results demonstrated that $22 \%$ of Indian students either faced or perceived to have faced some unfair treatment from the wider New Zealand community (when the responses under the categories of 'sometimes', 'often' and 'very often' are summed up). As compared to that, $19 \%$ felt to have been unfairly treated by the domestic students; $17 \%$ experienced or perceived to have been discriminated by their academic staff, as compared to non-academic staff (13\%) in the universities. It is also notable that the findings indicated some cases of unfair treatment from other international students $(8 \%)$. In total, the results demonstrated that those minority of participants who experienced some form of unfair treatment, it was more from the wider community, and less from the people in the education sector. Figure 6.4 displays the details. 


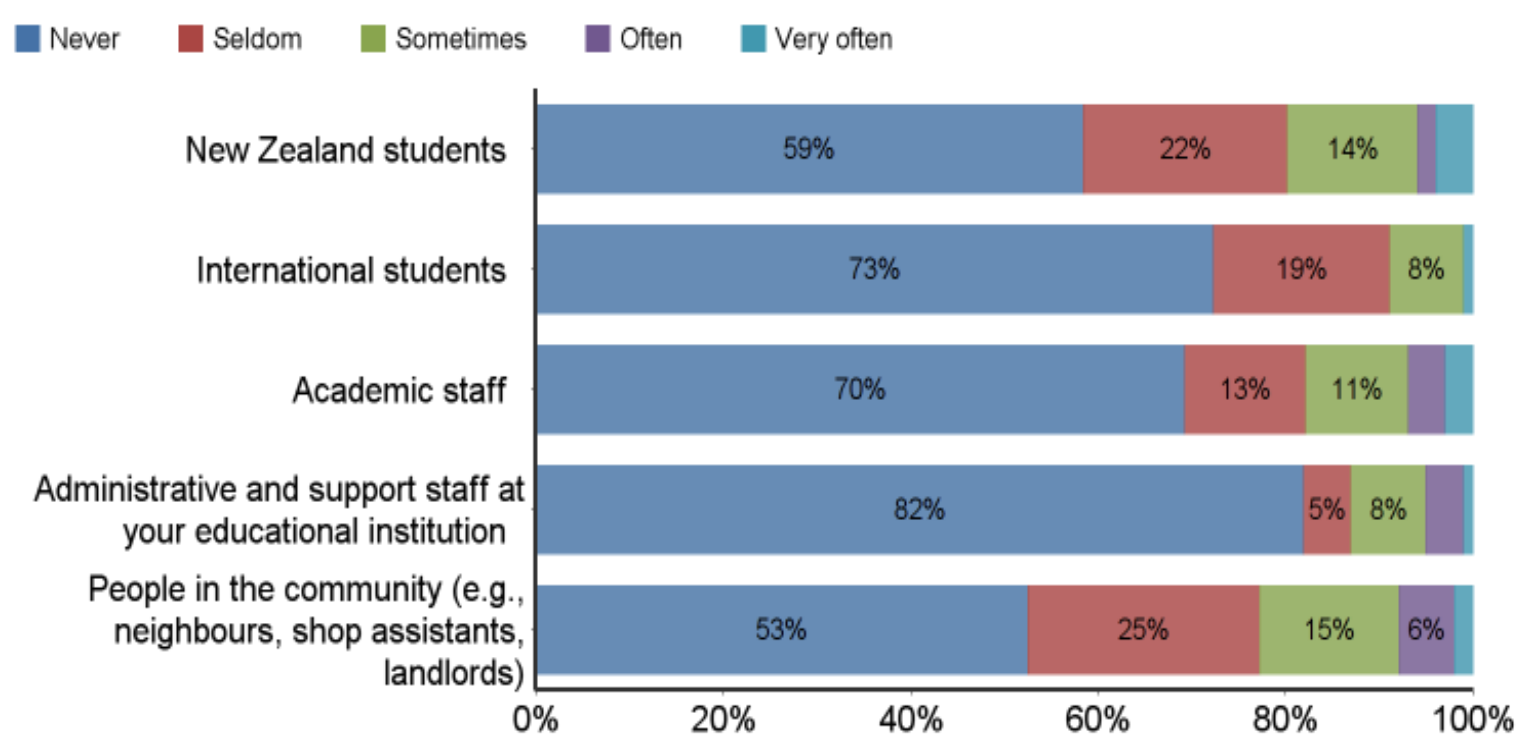

Figure 6.4 - Unfair treatment received from the following categories of people

The respondents of the survey were also given an open ended question to share their experiences, and for that 36 responses were reported. The following are some of the critical views expressed by them related to this issue. The perception of one respondent indicated traces of discriminatory treatment in their social life in New Zealand as shown below:

R.83: NZ is considered as an egalitarian society, I most certainly do not agree with this point of view. White people drunk in the street shout racial slurs all the time.

It appears that one of the respondents seemed to recollect his past memories and commented in the following way:

R.108: My programme director during my Honours course was a racist and was very unreasonable while dealing with me and caused me trauma and misused his position of power.

The following comment reveals the existence of unfair treatment even at higher level courses in New Zealand. 
R.21: One of my supervisors often degrades me and my country people because he had a bad experience in the past with some Indian students.

While such critical views were not shared by the majority of the respondents, they nevertheless indicate the existence of a problem, however minor that might be. Similarly, participants of the interviews were also asked about any unfair treatment they might have had either within or outside the university. The majority of the participants stated that they did not have any such unfair experiences. However, a few participants shared their unpleasant experiences related to this matter. Selected examples where participants felt to have encountered unfair treatment are given below:

Vineel: ...Some of the staff when I greet them they don't greet me back. I feel that because of my ethnic background they are doing like so. Sometime I got depressed because of this.

Another newly arrived student recollected a devastating experience which happened within a month after her arrival in New Zealand. She felt that it was a horrible experience and explained the situation in the following way:

Razia: One bad experience was in a bus. I was carrying stuff from a supermarket to my house. That day I blindly bought some stuff too heavy to carry it back home. That day I took a bus to get back home. I was new here and that was the same day I got my Snapper card. I have never sweated in this one month that I came to New Zealand, that day I was sweating badly and it was the worst day I have had in New Zealand. ... What happened was there are two doors, one in front and one at the back. When the bus stopped the second door was closer to me. Till that day I didn't know that I cannot enter the bus through the second entrance because I was new here. ... So I got in and immediately the bus driver who was a lady she got out of her seat and she wasn't really yelling but she was really rude. Ifelt like she thought that I probably would travel without a ticket. She was very hard and asked ... don't you know to snap your card in the front. I said I am new here and still she was rude to me.... She didn't really want to hear that from me. So that was one bad experience. 
The reported incident though cannot be described as a case of racial abuse or harassment, was nonetheless a matter of perception of being insulted in front of others. However, these odd incidents should be set into perspective by mentioning that the considerable majority of students never experienced any racial harassment.

\subsubsection{Opportunities for friendship with local students}

Out of the total of 102 responses received in the survey on this issue, $72 \%$ wanted to have more New Zealand friends; these include the categories of 'Mildly agree' and 'Strongly agree' responses put together. Similarly, 34\% of the respondents indicated that New Zealanders should attempt to make friendship with international students. Interestingly, 31\% thought that New Zealanders were not interested in having friendship with international students, while $41 \%$ thought otherwise. Likewise, while $44 \%$ of the respondents indicated that it was very hard to develop friendship with New Zealanders, a noteworthy percentage of respondents $(38 \%)$ differed from this view. Besides these observations, 55\% of the responses showed that these students tried their best to develop friendships with New Zealanders. In short, views and experiences of the Indian students on this issue of personal friendship with local people are almost evenly divided. The details are presented in Figure 6.5.

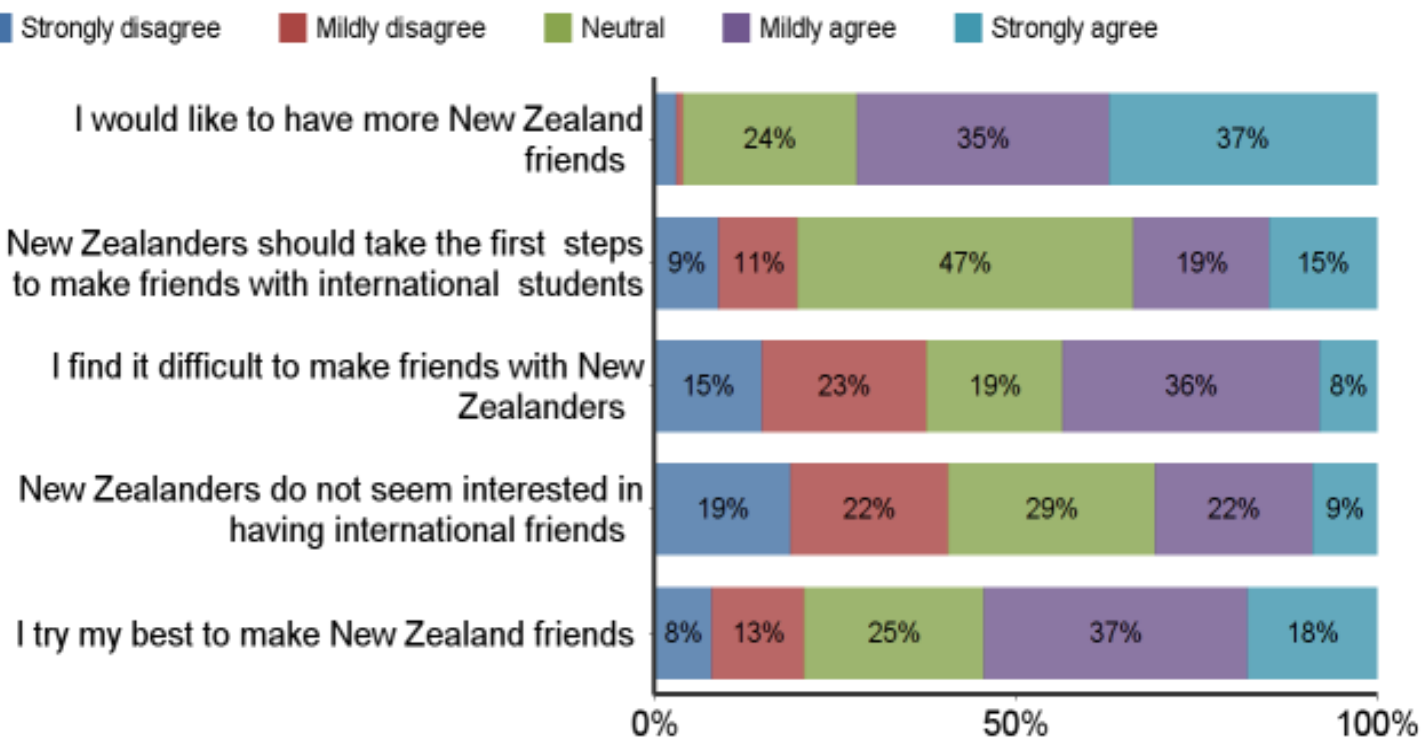

Figure 6.5 - Participants' desire to make friendships with New Zealanders 
The responses to similar questions in the interviews also revealed that these students had mixed experiences with their Kiwi friends. About eight participants out of 15 interviewed said that they had Kiwi friends, whereas others said that they had acquaintances but not friendships. Seven out of fifteen said that they had received help in academic and social matters from their local friends. One of the comments given by a postgraduate student is given below:

Vineel: Yes they do. Some time when I miss bus some of them do help in giving a ride to my home or in explaining any academic matters.

Another student when asked if any help was received from a local Kiwi friend at the time of need, he stated in the following way:

Sunil: I don't think so. It's just casual. The bonding thing which we had between individuals is very low here compared to India.

A similar view expressed by another interviewee is shown below:

Monish: You can't say its friends, but a guy is living with us. He is from NZ, we just say Hi, Hello, good morning, good evening, and that's it.

The perceptions of participants conveyed that their friendships with local peers was just casual and not as strong as their friendships in India, which is perhaps not unexpected.

\subsubsection{Opportunities for religious practices}

Having come from a multi-religious country, participants of the survey showed affiliation to many religions. Three quarters of 104 participants who responded to this question belonged to Hindu religion. The rest represented other religions, such as Christianity, Sikhism, Islam and Buddhism. A small percentage (3\%) of the respondents indicated that they did not belong to any religion, and an equally small percentage (2\%) did not wish to answer this question. The details are shown in Figure 6.6. 


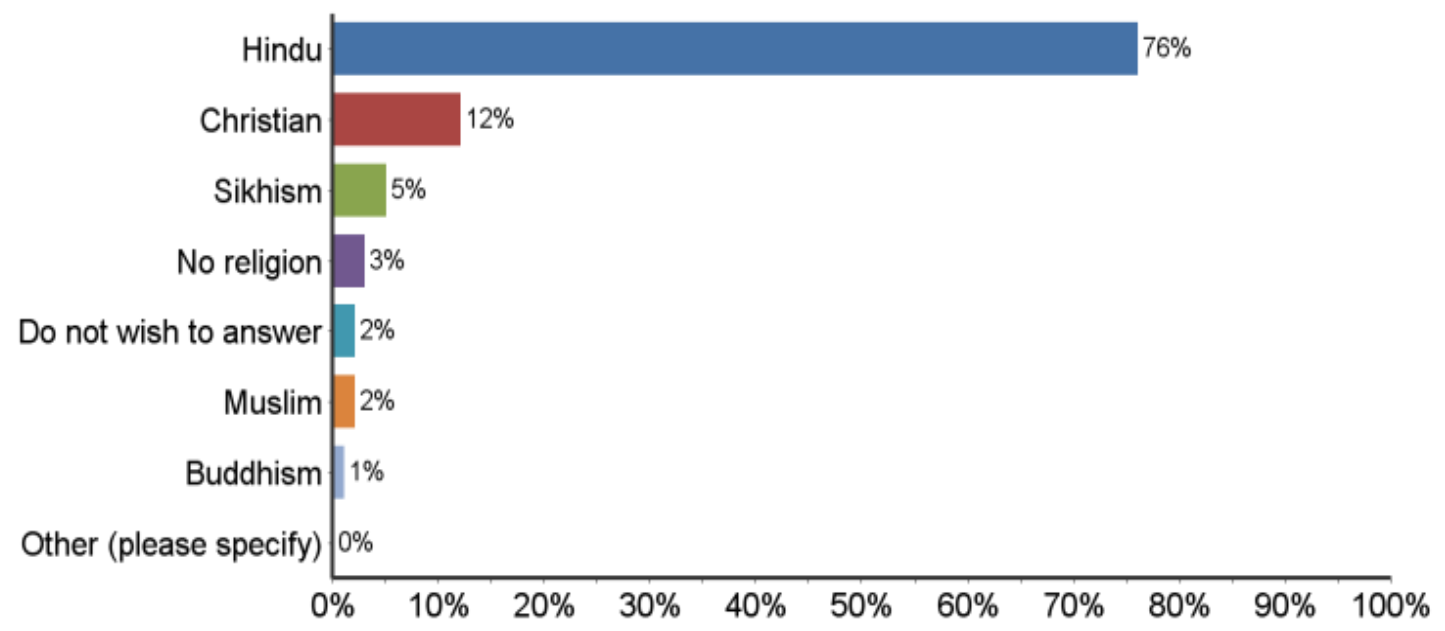

Figure 6.6 - Religious affiliation of the participants

In response to an open ended question in the survey many respondents (63) $(\mathrm{N}=83)$ stated that they did not face any specific difficulty in carrying out their religious practices in New Zealand. Some respondents revealed that they did not actively practise religion despite belonging to a specific religious group (11). A few others revealed that they did not wish to answer the question (5), while some specified that this item was not applicable to them (4).

Among the positive comments mentioned in the responses two of them are provided below:

R.78: Excellent, much similar to India.

R.67: It's good in this country as well. There are also gurudwaras and I go there.

One of the respondents indicated how religious practice is being utilised in finding friends in the following way:

R.16: Apart from the spiritual side, going to church here has enabled us to meet people and make new local friends. 
Another response associated with a little difficulty:

R.35: Not really satisfied with my religious activities when comparing with my activities in India. But still I should say that I am engaged in some kind of religious activity while living in here.

The participants of the interviews also confirmed the availability of opportunities for practising their religions.

Vineel: Yes, there are plenty of opportunities. If I feel depressed or down I would go to temple.

Similar to the participants of the survey, the obstacle specified by one of the interviewees was minor as he could not go to temples due to transportation problems.

Monish: I go to a temple... the bus to the temple runs at a specific time. It's quite far from the place I'm residing at. So there is only one bus which goes and its timing doesn't suit me and the bus doesn't run on the weekends. So I don't have any other way to go...

One participant informed that he was no longer practising religion in New Zealand.

Rajasekhar: I was raised as a Hindu and after coming to New Zealand I actually quit my religion. I don't have any religious views and I respect people's religious views and I have no issues with them. But I don't see any need for it in my personal life.

Having come from different religious backgrounds, the participants neither expressed any serious concerns, nor made any requests for special provisions to maintain their religious practices. It seems religion plays a limited role in their lives in New Zealand. Religious differences among Indian students therefore do not seem to be making any major differences 
in their experiences of adjustment in this foreign country.

\subsubsection{Satisfaction with life in New Zealand}

In the survey, $41 \%$ of the respondents who answered the question on their life in New Zealand $(\mathrm{N}=100)$ noted that their life here was according to their expectations and they rated their level of satisfaction in mildly and strongly agree categories. On the other hand, $37 \%$ of the respondents expressed a contrary view by opting for mild and strongly disagree categories. Then again, when the respondents were asked to specify the level of their life satisfaction, 50\% of the respondents by marking their responses in mild and strongly agreed categories reported that their life in New Zealand was actually satisfactory. Only $24 \%$ of the respondents showed their dissatisfaction by marking their responses in mild and strongly disagreed categories. Remarkably, $26 \%$ of respondents were still uncertain and indecisive and could not specify whether they were satisfied or not with their life in New Zealand. Figure 6.7 shows the details.

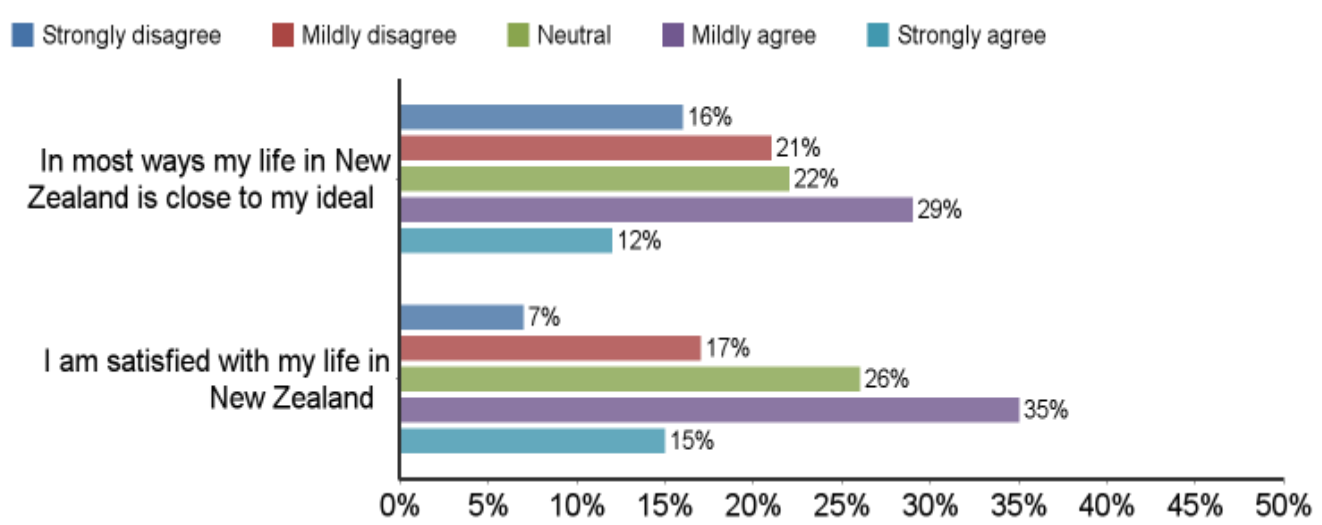

Figure 6.7 - Percentages showing satisfaction levels of life in New Zealand

The survey respondents were also given an open ended question to outline their comments related to this issue. The 46 responses expressed by them varied a great deal with both positive and negative responses. They mainly appreciated the academic aspects of their life in New Zealand and at the same time made adverse remarks about their non-academic or social life.

A respondent noted that though it was a challenging experience, the learning experience enhanced the levels of confidence and assured a better life. 
R.31: It's really challenging to be an international student in New Zealand cultural shock, different educational system, new friends, pressure from family, part-time work. Overall it was a great learning experience in NZ, but it's costly in Indian amounts. But I am confident ... the skills we acquire from NZ will help us to learn more and live life in a better way.

Another response emphasised the limited work opportunities available in New Zealand even though friendly environment and quality education facilities existed.

\begin{abstract}
R.5: Kiwis are great people, most of them make you feel welcome; the university is excellent in most ways too, but the fact is the Kiwi economy is not big enough to generate enough jobs for international students and a degree from New Zealand has little or no value in India. So I am not as delighted as I thought I would be after coming to New Zealand, still it has been a valuable experience.
\end{abstract}

Another comment indicated satisfactory learning experience, notwithstanding the sense of disappointment with low interactions with the Kiwi people.

R.79: It is a great feeling. New Zealand is a nice place with good education system. Food and other facilities are good. Just feeling Kiwi people are less interested to interact with us. Social life is poor. Living standard is high. Loneliness is there.

Similarly, in the individual interviews, at the end of each interview participants were asked whether or not they were satisfied with life in New Zealand. Out of the 15 people interviewed, 9 participants said that they were satisfied with the life in New Zealand, while two students said that they were only partially satisfied. Others commented that they were not satisfied with the social life in New Zealand. A few of the participants' comments are presented below.

For some like Mahima, the independence they enjoyed here was worthwhile:

Mahima: I wouldn't say I am satisfied, but I am happy the way I am coping with new things, being independent and taking care of oneself and being financially 
independent and doing part-time jobs, taking care of my living expenses as well as coping up with the new academic system. I am happy.

For others like Jasmine, the academic environment, and the clean and green nature seemed attractive:

Jasmine: As far as I am concerned I believe that I am quite satisfied. I expected it ... to be turning out like that. I am happy with the studies here, I am happy with the people and I find them quite helpful. Yes I have some friends who don't have good experiences over here...I consider myself lucky in that aspect that I quite like the study. One of the reasons is the support from my parents. They are quite supportive; there is no pressure on me to work. In general, I am happy with the country. It is really beautiful; it's clean and not polluted. I like the atmosphere because I am a quiet person and I like peaceful places and I am enjoying the life.

But for others like Pranith who came to New Zealand in the wake of Christchurch earthquake, the nature itself seemed frightening:

Pranith: Yes. However, in the first few days I was frightened by earthquakes and stuff. I didn't know ... that earthquakes ... happen so often here.

In total, this study revealed that $50 \%$ of the respondents of the survey (see Figure 6.7) and 60\% of the interviewees were clearly satisfied with their life in New Zealand. Likewise, this study also found instances of loneliness and isolation among Indian international students particularly in the initial months of their stay in New Zealand. Further, this study revealed that the majority of the Indian students perceived that accommodation provided by their educational institutions was very expensive. This resulted in only $7 \%$ of them choosing accommodation provided by their universities, while the rest preferred private arrangements. Around two thirds of the respondents were interested in getting a part time work, but a significant number of students could not achieve their goal. The leisure was mostly spent on social media activities and in fulfilling their academic tasks. A majority of respondents indicated changes in their activities during their stay in New Zealand. Students had ample opportunities for getting their Indian food, movies and music. A majority of the respondents perceived that their institutions 
respected their cultural differences. A few of them noted a few minor levels of unfair treatment mainly from the local community. Students desired for more interactions with the Kiwi people, but experienced low level of interactions with them. Finally, they did not reveal any serious issues with regard to their religious practices while staying in New Zealand.

\subsubsection{Personal impressions and suggestions for improvement of conditions}

The survey included a final question on whether or not they would recommend their friends and family members to study in New Zealand. A majority (60\%) of the respondents responded positively, while a minority (17\%) indicated that they would not. However, $24 \%$ of students were still in dilemma about their decision. The percentages can be viewed in the following figure 6.8 .

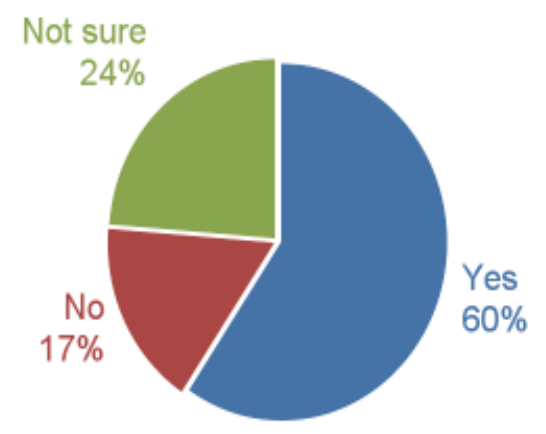

Figure 6.8 - Percentages of responses showing whether or not encouraging friends and family members to study in New Zealand

Further, when the students were asked to provide comments on their experiences of being an Indian international student in New Zealand, nearly half of them (46) responded. Most of the comments reflected a positive spirit, though there were some negative feelings too. A few of their comments are provided below.

One student was excited about the gain in personal development:

R.93: It was good to explore ... different cultures. I have learnt many things here and gained lots of confidence. 
Another student appreciated the egalitarian nature of the society:

R.36: It's easy. No racism. Everybody respects each other irrespective of religion, country, language, culture....

A female respondent was happy with the equal opportunities irrespective of gender:

R.27: Being an Indian female who is crazy about sports, I would like to say that here it is a place where you are given equal opportunity irrespective of your sex and you are encouraged to get better in whatever you do, if you are willing to work hard.

For a respondent lack of suitable job opportunities mattered most:

R.86: Not much productive place for high skilled jobs.

One comment stressed the need for effective communication skills:

R.56: It's a tough job, but you can manage if you have good communication skills.

One student pleaded for more proactive roles for universities and the community:

R.53: I think it would be easier for new students, if the university becomes a little more proactive in making them feel included, and hopefully locals too should become more receptive.

Another student made a suggestion to the prospective students:

R.98: It is very easy to say that I want to go to abroad for my further studies, but I strongly recommend everyone to do your proper homework before making your final decision, because life is not as rosy as it looks from outside.

Later, the interviewees also expressed mixed opinions. Nine of them positively responded and said that they would recommend friends and family members to study in New Zealand. Two said that they would not recommend because of limited job opportunities. Three other indicated 
that prospective students should evaluate the conditions such as financial abilities, job opportunities, and the selected courses of study before taking a decision. One interviewee was unsure of his recommendation because of limited work opportunities and less industrialisation of the country compared to India.

When the interviewees were asked at the end of interviews to make any suggestions for the improvement of conditions for international students in New Zealand, all of them utilised this opportunity to express their opinions. These were mostly targeted towards their universities, even though a few were intended for prospective international students.

An undergraduate interviewee gave a suggestion to the universities emphasizing the diverse nature of issues:

Dinakar: One big thing would be just don't generalise every international student have same issues. Make it more like one on one case. I mean if someone from India will have a problem different to the problems of a person who is grown up in China.

A postgraduate course work student asserted the need for enlightening the international students on the academic expectations of the New Zealand educational system:

Vineel: I feel that there should be a strong orientation on the academic patterns and assessment systems as there is a major difference in the education system.

Another interviewee emphasised that the prospective students should do thorough research prior to their arrival in the host country.

Pranith: From students' perspective, one should do research to know the facts and history of the university before coming to the university. After coming to the university it is over. I don't know how many students are doing but I did it and I felt like secured. 
A master's student Suresh, recommended monitoring of the activities of the educational agents by the universities.

Universities should oversee the consultancy agencies by a body or organisation that whatever information is being provided is correct and authentic. It's not all sunshine and flowers like they said here and life is tough and you have to struggle to find a part time job, you then have to struggle to live alone.

A female doctoral student interviewee stressed the requirement of effective orientation and guidance from the universities.

Rashmitha: There should be some guidance on the provisions of services available initially at least. I came to know about the services through some other students but not through the university. There should be orientation for the PhD students as well. My supervisor just took me to the whole of the building and that to see the labs. I still don't know who are there in the department.

The other suggestions included the requirement of support concerning accommodation, career guidance, and continuous assistance in academic and other aspects.

\subsection{Summary}

This chapter has presented the experiences, perceptions and views of the Indian international students related to their personal and social life in New Zealand. With regard to their living arrangements most of them were found to be living in private accommodation and perceived accommodation provided by their universities to be expensive. Concerning their part-time work, students generally opined that it was hard to get a work opportunity and stated several reasons. The participants indicated many changes in their leisure time activities, but on the whole they enjoyed life, maintained their cultural and religious practices, and maintained their social networks and regular connections with their family and friends back home. A minor level of discomfort was noted by a few students related to unfair treatment. Once again, their low level of interaction with local students was affirmed in this study in line with the past studies. The most important finding of this research is that the majority of participants were found to be satisfied with their life in New Zealand. Moreover, many of them expressed their willingness to recommend their friends and family members to study in New Zealand. Finally, participants of the study made some suggestions mainly to the universities and a few to the prospective international students. The next chapter will provide discussion of all the findings of this study. 


\section{Chapter 7 \\ Discussion}

\subsection{Introduction}

In this chapter findings from the study are interpreted and discussed in relation to previous studies of the international student experiences and theories of adjustment. Attention is paid to the themes and factors identified in Chapters 4-6, specially the reasons why Indian international students chose New Zealand as their study destination, English language proficiency, learning experiences in a different educational system, experiences related to the support services in their educational institutions, and the social life experiences in New Zealand. Further, the experiences of Indian international students related to recognised themes will be examined through the lens of four major factors impacting on of adjustment process: Support Services, Language proficiency, Friendships, and Information (SLFI), as they were identified in the literature review as key components of psychological, socio-cultural, and academic adjustment. By relating the four major factors to the experiences of Indian international students this study will highlight their current conditions in New Zealand, thereby alerting to any needs or provisions required for a positive adjustment.

Furthermore, it is anticipated that this discussion will also identify the current challenges faced by the Indian international students, and subsequent strategies applied by them in addressing these issues for better adjustment. Other than these, a model programme is suggested to facilitate better adjustment of international students in New Zealand or elsewhere by integrating the findings of this study and the recommendations of the related literature. Finally, this chapter will be concluded with a brief summary of important aspects discussed in this chapter.

\subsection{Factors influencing choice of a study destination}

The study first of all tried to explore the factors which influenced the Indian international students' decision-making in selecting New Zealand as the country where they wanted to 
pursue their higher education. This discussion can shed light on some of the expectations with which international students arrive in the host country. When these expectations match later experiences, it reduces challenges, as well as helps better adjustment and academic achievement. The existing literature has also alerted us to the need for considering factors, especially, at a pre-entry stage that influence the choice of study destination to know the trends of global student mobility. So, an attempt was made in this study to consider the perspectives of Indian international students at the time of their decision making.

When the participants of the survey were asked whether or not New Zealand was their first choice of study, 55\% of the students affirmed positively. The rest of the respondents mainly indicated countries such as the USA, Australia and the UK. In response to an open-ended question on what influenced their decision making, the largest group (29\%) of responses indicated that good courses, quality education, university ranking, good faculty and interesting courses or projects, or in other words, quality of education and reputation attracted them to New Zealand universities. The next group of responses (28\%) mentioned that peaceful and safe environment, friendly people and relaxed life style were other points of attraction for them. The factors indicated in these responses are generally the same which attract a majority of international students to New Zealand. The past studies in New Zealand have highlighted these aspects from time to time (Business and Economic Research Limited, 2013; Deloitte, 2008; Ward \& Masgoret, 2004). However, in our study $21 \%$ of the responses indicated that low fees and scholarships have also been major draw cards.

Observing the phenomenon of student mobility, a past report indicated that host countries have vested interests in international students, especially because of short term and long term benefits (Verbik \& Lasanowski, 2007). So, it was noted earlier in the literature review, that host countries would provide incentives to attract international students. Such incentives seemed to have impacted on some Indian international students who chose New Zealand as their study destination. For example, for a number of Indian students the award of financial support or scholarship played a major role in making their study choice of New Zealand. Out of the total 105 respondents to the survey, 23\% reported that they were awarded scholarships for their study. Another $15 \%$ described themselves as doctoral students with domestic fees. Among these total students, 57\% were involved in their doctoral or master's degrees, which indicates $67 \%$ of them were financially aided either partially or fully. A total of fifteen 178 
responses in the survey specifically indicated that award of scholarship was the only reason behind their choice of study destination. Similarly, six responses indicated that charging of domestic fees for doctoral studies influenced them to study in New Zealand.

Similarly, among the interviewees, all the participants engaged in doctoral studies (40\%) specified that the award of scholarships or financial support was the only criterion for choosing New Zealand as their study destination. The present study shows that the Indian students particularly those who come for postgraduate studies - give due importance to the provision of financial support or scholarships in making a decision on their study destination, and this view seems to be unique to this specific group of students. In fact, this finding is in contrast to the 2003 national survey in New Zealand which indicated that offer of scholarships and financial support was of less importance to international students while selecting a study destination (Ward \& Masgoret, 2004). This indicates that the needs and preferences of each international student group hailing from a different background can be dissimilar to those of other international student groups.

This will become clear if we look at the financial situation of these students. In response to a question in the survey on the level of difficulty to meet their educational expenses, a majority of the respondents (75\%) reported that it was ('moderately' to 'extremely') hard for them or for their families to meet their educational expenses in New Zealand. In the interviews a few students indicated that they came to study in New Zealand because, for example, it was 'one of the cheapest places to study when compared to other developed countries'. Particularly, domestic fees for doctoral students had been a major draw card, as among the total respondents $15 \%$ specified that they were paying domestic fee for their doctoral studies. Altogether it implies that for some of these students at least, less financial burden compared to other destinations encouraged them to undertake their studies in New Zealand. It should also be noted that a majority of the Indian international students in this study were postgraduate students, and only eighteen percent of them were involved in undergraduate studies.

Apart from the financial support, the decision about study destination for some Indian international students was also influenced by other factors such as future work opportunities and perceived easy immigration policies. In the survey $14 \%$ of responses indicated that students 
anticipated future work or immigration opportunities at the time of their decision making on a study destination. Such expectations are in line with the past observations in the literature, which identified that at the time of decision making prospective international students considered both short term goals of education and long term goals of future career and migration prospects (Baas, 2010; Bandyopadhyay, 2010; Bethel \& Ward, 2014; Doyle et al., 2010).

Besides these considerations, a few personal factors also played a role for Indian students in their decision-making. Some of the factors are: a personal interest to study abroad, previous visit to the country, influence of friends and family members who are already staying in New Zealand. This situation implies that the decision concerning study destination was influenced by either personal interests of the students or by others' advice. These findings confirm the views of a few studies. Chen and Zimitat (2006) in their study on Taiwanese students specified that the personal attitudes and behaviours of individual students influenced their decisions for studying abroad. Likewise, Ho et al. (2007) study on Chinese international students confirmed that parents of the students significantly influenced international students' decisions to study abroad. Moreover, a few responses of Indian students indicated that racism in other countries or a lack of alternative choices to consider, forced them to select New Zealand for their study. In other words, the unfavourable conditions existing in other countries also favoured New Zealand to increase its international student numbers.

Among all the factors mentioned by Indian students, interestingly, only one participant of the survey, and one interviewee (might be the same person who participated in both the survey and interviews) stated that the English speaking environment of New Zealand attracted them to this country. This finding significantly varies from the studies on Asian students in general as well as from the national survey reports of New Zealand in 2003 and 2007 which indicated that the English speaking environment of New Zealand was a major influencing factor for international students. However, it must also be acknowledged that only a few Indian international students in this study indicated that they might consider a non-English speaking country, such as Germany, for their higher studies.

The findings on the decision making of Indian international students provided a perspective on the participants' expectations from New Zealand. It was also noted that some of the 
expectations were exclusive to Indian international students (e.g., scholarships and financial constraints), whereas other expectations were common for all other international students (e.g., high quality of education, the image of New Zealand as peaceful and safe country). However, it should also be noted that the majority of our respondents were postgraduate students for whom scholarships and domestic fees were important. When the experiences of international students in the new host country do not correspond to the expectations, it can result in significant challenges and may further impact the adjustment process and academic success of these students. To avoid these adverse experiences, specific strategies to minimise these gaps or to overcome their challenges need to be applied by the students to reach their predetermined academic goals. The next section discusses the role of the support services and their impacts on the Indian international students.

\subsection{The role of support services in the adjustment of Indian students}

One of the significant factors that influence the adjustment process of international students is the provision of care a person receives from the support services of their educational institution. The primary purpose of support services is to address any challenges faced by international students in a new environment. By equipping an international student with necessary knowledge and skills, particularly in the challenging areas of psychological well-being, social life, and in academic matters, support services in an educational institution can positively influence the adjustment process.

The findings of this study suggested that the majority of the Indian international students who participated in this study adjusted well to the new academic conditions and social environment in New Zealand. And here their experience differs significantly from those of the other Asian international students. However, having said that some respondents $(10 \%)$ to the survey as well as a few (2 out of 15) participants in the interviews also admitted facing some psychological challenges, such as loneliness and isolation, as sojourners in a new place, confirming the similar arguments made in previous studies (Khawaja \& Dempsey, 2007; Sandhu, 1994; Ward et al., 2001). However, the difference lies in the magnitude of this problem, as majority of the Indian students we surveyed did not indicate any such problem. In 
this context, the previous studies suggested that language proficiency and friendships were necessary components for a better psychological adjustment. Furthermore, these studies highlighted the role played by support services in equipping international students in both these aspects. Here too Indian students offer a different experience.

Overall, the Indian students in our study reported better language abilities as their higher than required language test scores reflected. The only problems some of them faced were related to local accent, unfamiliarity with local idioms, and Kiwi style of humour. The aspect of language will be dealt in detail in the next section. It is sufficient to note here that the knowledge of English language of an individual can be different from its local usage or informal daily life contexts. Hence, any sort of guidance or assistance towards the language aspects in terms of local usage or informal contexts by the support services could significantly improve the quality of their experiences in New Zealand, particularly in the initial months. One form in which international students are able to receive language assistance is through dedicated language centres in their educational institutions.

Besides the language aspects, friendships may contribute to dealing with psychological challenges such as loneliness and homesickness. In the survey, a majority (55\%) of the respondents noted that they have worked towards fostering friendships with host nationals. However, at the same time, a significant group of Indian international students (72\%) expressed a desire to have friendships with the host nationals. This situation indicates that the efforts made by the Indian students did not seem to bear fruit as the desire strongly resonated among many of them. Even in the interviews students made requests for support services to organise outings, or social gatherings or to observe cultural festivals which could create opportunities for mutual understanding and social interactions. Such suggestions were made because participants sensed increased interactions would minimise homesickness, isolation and loneliness. The aspect of friendships will also be dealt with in detail in another section, however, it is sufficient to add here that support services could play a vital role in creating multiple avenues for fostering friendships. The opinions expressed in this study find agreement with the previous studies which argued that support services should actively design special programmes that could foster social interactions, thereby minimising the psychological impacts such as loneliness, isolation and homesickness (Kwon, 2009; Spencer-Oatey \& Xiong, 2006). 
However, the students surveyed did not suffer from any such adverse consequences because of the impact of the internet on social networks. When the respondents of the survey were asked to indicate if they knew of any sources of support available in New Zealand in case of experiencing loneliness or isolation (Q. 57), surprisingly, only a few Indian international students (4\%) mentioned any knowledge of support available from the staff of their universities. However, the developments in the communication technology helped many of them to maintain continuous contacts with their friends and families and enabled them to acquire sufficient levels of emotional support. Almost three quarters of the respondents used social media daily, and another $7 \%$ weekly. Three quarters talked to their family and friends over Skype at least weekly or more frequently. This would suggest that the internet is playing a role in the connectedness of Indian international students. This suggests a very different international student experience to that of one or two decades ago when students were a long way from home, and it was expensive to phone home to talk to friends and relatives.

It is possibly because of this connectedness that the Indian students are less concerned about the support services from their universities. For example, for a different question in the survey on the usage of support services in the educational institutions, a majority of the Indian international students (97\%) reported utilization of various services and a majority of them $(83 \%)$ also expressed satisfaction with those services. Yet, only a few would know that help was available from their educational institutions to deal with their emotional stress during troubled times.

The existing literature suggests that psychological challenges may lead to negative impacts on the academic adjustment as well, in some cases it may lead even to withdrawal from the course (Poyrazli \& Lopez, 2007; Thurber \& Walton, 2012; Tochkov et al., 2010). Similar findings of a few studies appropriately encouraged educational institutions to take an active role in dealing with psychological issues encountered by international students (Khawaja \& Dempsey, 2007; Sandhu, 1994; Sawir, Marginson, Deumert, Nyland, \& Ramia, 2008). Even though studies encouraged educational institutions to provide support, Indian international students reported low levels of utilisation of specific support services. Interestingly, such a low utilisation in the case of Indian students did not hinder their academic satisfaction and achievement, as they were able to receive support from other sources. The findings also indicated that participants 
received support from co-nationals and other international students to deal with any concerns. This partially confirms the findings of Ward and Kennedy (1993) that co-national support minimises psychological challenges.

In relation to socio-cultural adjustment, the findings indicated that a significant number of participants were either unaware of or perceived no services were available in their educational institutions. To state a few examples, a considerable number of participants stated unawareness in the following areas: buddy or mentor programme (around 42\%), counselling services (34\%), financial advice services (34\%), vocational (career) or educational guidance (29\%), learning support services (26\%), and accommodation (21\%). Unfortunately, a lack of awareness of support services resulted in difficulties in some cases. This study identified that the reason for such unawareness was the impact of cultural differences of the participants, particularly when they did not attend orientation programmes. The failure to attend orientation programmes resulted in struggle and challenges in a new environment. This phenomenon confirms what is noted in the literature that a lack of knowledge of the existence of support services could lead to difficulties (Ang \& Liamputtong, 2008; Campbell \& Li, 2008; McKinlay et al., 1996; Sawir et al., 2009a, 2009b; Ward, 2001).

In a few instances Indian students stated that they were not 'told of' the availability of facility or service, and hence could not use that service. A lack of 'oral guidance' contributed to the ignorance of the participants regarding the available support services. The significance of oral guidance will be further discussed at a later stage; it will suffice here to add that there is a need for understanding the specific requirements of these students coming from diverse cultural backgrounds, and to apply appropriate strategies to provide information to them.

Apart from this, some interviewees did not attend orientation programmes either because of their unawareness of the value of information provided in these programmes, or due to late arrival in New Zealand. In order to address this situation, some studies recommended the need for making information available through a continuous comprehensive support system (Chun \& Poole, 2009; McKinlay et al., 1996; Zhang \& Goodson, 2011). The responsibility of providing information regarding the existence of support services and providing information on the socio-cultural differences of the host country is supposed to lay with the educational institutions (Mehdizadeh \& Scott, 2005). 
In this context, it is also necessary to note that Indian international students did not make any requests for special provisions of food or religious prayer, to be made available through support services. This is possibly because Indian international students are heterogeneous in their religious backgrounds, food habits and cultural practices. In fact, most participants commented that they had no issues or requests in this matter and did not convey an expectation from their educational institutions to cater for these needs. Such reports are in sharp contrast to past studies which insisted on special provisions for international students in the areas of religion, food and cultural aspects (Alazzi \& Chiodo, 2006; Mehdizadeh \& Scott, 2005; Novera, 2004).

Although Indian students did not make any requests for special provisions related to their food, cultural or religious practices, a closer look at their requests indicate a different kind of expectations. For instance, a majority of Indian international students were reported to be in private accommodation arrangements and they perceived the costs of accommodation provided by their universities were expensive. One of them suggested discounted accommodation provision by their universities. This situation indicates that they considered these provisions from their universities as valuable, but could not afford the costs involved. Similar opinions were expressed about the services of career support as well. While these provisions are available in their universities, some of the Indian students, indicated that their expectations were not met by them.

It is also worthy to mention that a majority of the students $(69 \%)$ in the survey indicated that no support was received from any of the Indian community groups in New Zealand. Further, during the interviews, some of the interviewees stated that they were not even aware of any such services being provided by any Indian community organisation. However, despite such inadequate support in matters of specific concerns, the participants reported better adjustment to a new life in New Zealand. A majority of the students stated that even though they experienced difficulties, particularly during the initial period of stay, gradually they were able to adjust to their new host country.

Even in the realm of academic adjustment, a majority of the participants reported that they were satisfied with the academic experience in New Zealand. The significance of the satisfaction needs to be viewed in the light of challenges some reported due to academic 
cultural variances in New Zealand. Challenging areas included academic workload, teaching practices, assessment procedures, communicating with their teachers, and interaction with their peers. Some of the existing studies have also alerted us to these cultural challenges (Campbell \& Li, 2008; Durkin, 2008; Eaves, 2009; Nayak \& Venkatraman, 2010; Novera, 2004).

The findings of this study revealed that Indian international students too did struggle initially due to these academic cultural variances. Some participants stated a few challenging experiences with their academic activities, and reported that these challenges were because of the differences between the educational systems of India and New Zealand. Some of the challenges indicated by the participants of both the survey and interviews were: writing assignments, completing the academic tasks within the time schedules, working on group projects, giving oral presentations, and managing their study workloads. A majority of students (73\%) revealed that these kinds of educational activities were unfamiliar to them. One third of the respondents of the survey reported that they had encountered a difficulty with writing assignments. Interviewees highlighted some of the challenging concerns such as referencing, plagiarism, and developing an argument. The challenging experiences of these students with particular aspects of educational activities reflect the general experiences of international students, who arrive at a different educational system. Based on their experiences Indian international students perceived that some support at the initial stages of their studies could be effective in reducing the stress. The views of these students were in agreement with the recommendations of the previous studies, which also claimed that pre-entry information to international students could ensure a better academic adjustment (Berno \& Ward, 2004; Mehdizadeh \& Scott, 2005; Nayak \& Venkatraman, 2010). In equipping these students with adequate information, the role played by the support services is quite obvious.

In addition, Indian international students expected support to be offered for enhancing their academic skills. When interviewed some participants requested support to make them aware of these academic norms and to enhance their skills. The aspect of offering specific help to international students was stressed in the past studies as well, which recommended that host countries should seek ways to provide adequate support for struggling international students (Andrade, 2006a, 2006b; Campbell \& Li, 2008; Eaves, 2009; Johnson, 2008; Millar, 2009; Soosay, 2009; Turner, 2009). The suggestions included providing extra support in the form of workshops or specialised seminars for equipping them with necessary information on different 
academic conventions, expectations, and practices. Moreover, studies also suggested that the focus of support services should be on enhancing individual abilities (Khawaja \& Dempsey, 2007). Interestingly, the nature of the requests of Indian international students in this study were also similar.

What is interesting however about the Indian international students is that they are able to cope with the challenges very quickly. Most of them seemed to be satisfied with the level of support they received from their respective universities in New Zealand. Some of the interviewees responded positively on the help they received from the Student Learning Support Services, particularly in improving their writing skills. One interviewee responded that by attending postgraduate workshops he was able to enhance his skills towards issues connected to writing and referencing. Another postgraduate student stated that he made use of the services of the library staff in receiving proper information or web links to improve his abilities and to overcome his academic barriers.

In addition, two participants revealed that they were able to receive help from their tutors, lecturers and professors either through feedback or seeking clarifications from them while dealing with certain academic issues. Many students perceived that the student-teacher relationship in New Zealand was different from that of India. A majority of the students reported that their teachers understood their problems and made special efforts to help them. Students described teachers in New Zealand as more accessible, friendly, and they encouraged them in achieving their academic goals. Students seemed to enjoy more freedom in contacting their teachers or in expressing their views or ideas. Most of the respondents of the survey $(77 \%)$ agreed that they felt included in their classes and reported working well with their classmates.

The experiences of those interviewed suggest that until they became familiar with the academic requirements they did not do well. However, they acknowledged that once they became familiar with the academic expectations, they were able to cope with the requirements. A better academic adjustment is reflected in this study, since most respondents to the survey (77\%) reported doing well in their academic studies. Likewise, many students (87\%) expressed moderate to extreme levels of satisfaction with their academic progress. The adjustment levels of these students differ from much of the research on international students, particularly those 
related to Asian international students (Campbell \& Li, 2008; Mehdizadeh \& Scott, 2005; Novera, 2004). These studies reported that differences in academic culture seriously impacted the learning process of Asian international students and recommended better support provisions to these students.

To illustrate this point further, although the academic environment, educational practices and expectations were very different to those they were accustomed to, most of these Indian students appeared to have adjusted to the differences, and enjoyed the new and different approaches to learning and assessment. Some of those approaches mentioned were: practical approaches in learning process, giving presentations, participating in discussions and group work, continuous updating of the curriculum, use of technology in teaching and learning process, student-teacher relationships, and even assessment procedures. Appreciation of these educational activities, even though these are unfamiliar and of a different kind, means that these students have recognised their value. This observation is similar to the observations of $\mathrm{Vu}$ and Doyle (2014), who reported that the negative perception of participants of their study due to academic cultural differences was changed to positive one, after they realised the value involved in those academic approaches.

Hence, the Indian international students' learning satisfaction in New Zealand together with a sense of appreciation of the academic cultural variances suggest that the support they have received during the course of their academic studies has helped them in their better academic adjustment. Nevertheless, their suggestions indicated that provision of support during the initial stages of their arrival would minimise their struggles early and allow an easier adjustment. Overall, the individual abilities and attitudes to adapt to the new academic environment, and better utilization of the support services resulted in better academic adjustment of Indian international students in New Zealand universities. This finding confirms the observations of Olivas and $\mathrm{Li}$ (2006). Therefore, in light of the above views it becomes essential not to underestimate the value of support services and the information they offer for a better academic adjustment of international students. 


\subsection{The role of English language proficiency}

Having considered the role played by support services, it is appropriate to focus on the next significant factor that impacts adjustment process: English language proficiency. In order to discern experiences related to English language proficiency the participants of this research were asked a few questions in the initial online survey and again in the individual interviews. The main intention was to assess their level of language proficiency through achievements in their academic and non-academic life, in an English speaking environment. The findings of the study indicated that about $80 \%$ of the participants did very well in the language proficiency tests required for admission procedures, approximately $87 \%$ were previously educated in the medium of English, and around 73\% 'often' used English language in their previous work contexts. This indicates that Indian international students arrive in New Zealand with better levels of English language proficiency than other Asian students. According to some previous studies, English language proficiency should decrease stress, therefore help in positive psychological adjustment (Duru \& Poyrazli, 2007; Poyrazli et al., 2004; Sümer et al., 2008; Zhang \& Goodson, 2011). In agreement with this observation, findings of this study confirmed that better language proficiency levels of Indian international students contributed to minimise their stress at least on the language front. The participants reported that their language proficiency helped them in their academic achievements (96\% rated it in good and excellent categories) and social life (94\% rated it in good and excellent categories).

However, the participants also noted some initial difficulties in the language front, and these included understanding a different accent, local idioms, and Kiwi style of humour. While conversing with others the participants had to adapt their communication style by reducing the influence of their mother tongue in their use of English language, slowing down, and using body language to communicate effectively. The importance of individual efforts, especially in the realm of English language proficiency, for a better psychological adjustment was noted in the study of Wang and Shan (2007). However, it is important to note that Indian international students did not report any major psychological concerns related to this aspect.

In the social contexts, participants attributed their language proficiency to a previous English speaking work environment, and past travelling experiences. The challenges of participants in 
social contexts also related to understanding the local accent, adjusting their pace of speaking and understanding the Kiwi sense of humour in conversational contexts. The impacts of these challenges in the socio-cultural adjustment could be huge, because, this could cause lack of friendships with locals (an essential ingredient of socio-cultural adjustment) and thus hamper socio-cultural adjustment. But interestingly, when asked if the language difficulties prevented them from fostering friendships with domestic students, only $11 \%$ of the participants agreed with this view, while a majority $(75 \%)$ of the students did not consider it to be a major obstacle. The finding thus endorses the views of some of the previous studies which argued that better language proficiency helped in building friendships with the host nationals, thereby positively influences socio-cultural adjustment (Andrade, 2009; Campbell \& Li, 2008; Poyrazli \& Grahame, 2007; Swami et al., 2010; Zhang \& Goodson, 2011). This finding however could be further explored in future studies.

In the academic sphere too, as stated above, the Indian international students self-rated their communicative abilities very highly. If there was one area where a few of them (11\%) reported challenges, it was with academic writing and its protocols. These statistics reflect the effectiveness of their previous education, wherein they undertook academic studies in the medium of English. In this context it may be noted that an empirical study by Novera (2004) on Indonesian postgraduate students in Australia reported that out of twenty five participants twenty three reported English language difficulties in academic context. Apart from that other studies too suggested that international students, particularly from the Asian region struggle academically due to poor English language skills (Campbell \& Li, 2008; Holmes, 2005). By comparing these findings with the results of the current study, it could be suggested that the experiences and needs of a particular group of students hailing from a specific region might be different from those of other groups of students. Therefore, it requires appropriate strategies and responses to deal with different groups of students, having diverse sets of requirements.

In addition, past studies highlight other challenges experienced by international students in academic contexts due to a lack of English language proficiency (Andrade, 2009; Campbell \& Li, 2008; Henderson, 2009; Holmes, 2005; Li et al., 2010). These include: classroom participation, understanding the lectures, oral presentations, communication with teachers, working in the group projects, and attendant writing tasks. However, in this study, when these aspects were considered (Q. 27), the responses in the columns of moderately to extremely 
difficult categories indicated that Indian international students faced fewer difficulties, as only a few (16\%) reported struggles in classroom participation. Likewise, only $7 \%$ reported difficulties in understanding lectures, $25 \%$ in giving oral presentations, $10 \%$ in communicating with their teachers, $21 \%$ working in the group projects, $36 \%$ in attending to writing tasks. The results therefore confirm that adequate levels of English language proficiency can contribute to less struggles in academic aspects.

The importance of English language proficiency in the learning experiences of international students has been emphasized in a number of studies (Poyrazli \& Kavanaugh, 2006). Given its significance, educational institutions require evidence of language skills through tests (IELTS or TOEFL). But even then, studies indicated that international students who acquired sufficient scores (or completed EAL requirements) still struggled academically due to intricacies of the language (Campbell \& Li, 2008; Holmes, 2005; Johnson, 2008; Wang \& Shan, 2007). The Indian international students also struggle with the local use of English language (in New Zealand context) as already noted above. But they did not report academic difficulties arising out of it; instead, they reported that the issues were quickly resolved in a short time of their stay period. So, the arguments of Holmes (2005) as well as Wang and Shan (2007) regarding the raising of IELTS score requirement to address English language difficulties could then be re-visited, taking into account the fact that the language abilities are particular to the context of the place as well as the background of specific international student groups. Instead, as Andrade (2006b) and Poyrazli and Kavanaugh (2006) have argued, assistance should be made available to such students through providing opportunities to improve their existing language skills, and to promote interactions with native students. As Khawaja and Dempsey (2007) have suggested, support services in the educational institutions should take a proactive role by focusing on equipping the international students with necessary knowledge and skills.

\subsection{The role of friendships}

The third aspect to consider while discussing the experiences of international students is about friendships, particularly, the impact of friendships on their adjustment process. The data revealed that some of the Indian students struggled making friendships with the domestic students, but there were also others who had local friends. When asked about making domestic 
friends in New Zealand, $44 \%$ reported that it was difficult to make friendships, while 38\% differed from this opinion. In the interviews seven out of fifteen participants reported that they did not have any friendships with local students. These findings, which indicate that opinion on local friendship was almost evenly divided, thus only partially confirm observations of earlier studies which asserted that international students struggled to make friendships with domestic students (Ward, 2001; Ward \& Masgoret, 2004). Our findings also indicated that while the respondents (72\%) had a strong desire to have more New Zealand friends, a few $(31 \%)$ indicated that domestic students were not interested. These findings again only partially correspond to the findings of other studies (Ward, 2001; Ward \& Masgoret, 2004).

This Indian attitude to friendship had other interesting dimensions. When Indian international students were asked about the people they consulted during times when they felt upset, troubled, lonely, and homesick, a sizeable section (47\%) reported that help was sought from the people in their home country. Apart from this a few approached fellow international students (23\%), and only a few of them (3\%) depended on domestic friends for support during times of psychological challenges. The existing studies suggest that friendships could positively influence psychological adjustments, since friends could relieve a person from the feelings of loneliness and isolation (Kwon, 2009; Spencer-Oatey \& Xiong, 2006; Wang \& Shan, 2007; Ward \& Kennedy, 1993). Apart from the psychological aspect, it was noted by the previous studies that friendships or intimacy with others in the host country significantly minimised the socio-cultural difficulties (Brisset et al., 2010; Ward \& Rana-Deuba, 1999). Moreover, studies also suggested that co-national support could also serve a similar function by relieving international students of feelings of isolation and homesickness, especially when domestic friendships were lacking (Wang \& Shan, 2007; Ward \& Kennedy, 1993). However, the Indian international students in our study even though they indicated lack of friendships with domestic students, they did not report any major psychological challenges, as majority of these students had access to their friends in India through social networking sites. It shows how the internet and social media keep the international students connected to their friends and family back home, and reduce their loneliness and psychological stress. These findings force us to rethink the role of local friendships in the lived experiences of international students who now live in a globally connected social network. 
The respondents in this study also mentioned a few factors that hindered their friendships with local students. These are perceived discrimination, perceived cultural distance (because domestic peers viewed them through stereotypes), lack of financial resources (restricted them in engaging in aspects such as choosing university accommodation), a lack of interest in activities (such as sports and recreational activities), and a lack of own transport. These observations are consistent with the previous studies which also highlighted perceived discrimination (Berno \& Ward, 2004; Hanassab, 2006; Lee \& Opio, 2011; Lee, 2010; Lee \& Rice, 2007; Novera, 2004; Poyrazli \& Grahame, 2007; Swami, 2009; Swami et al., 2010), perceived cultural distance (Swami, 2009), financial resources (Swami et al., 2010) as factors hindering contacts between the international students and domestic students. However, besides those challenges, the current study revealed that English language was not a hindrance for developing such friendships; as only $11 \%$ thought that it was.

A majority of them (55\%) reported that they made efforts to make friendships with local students. Apart from those, some participants (25\%) opted for neutral response, a few others (20\%) indicated that they had made little or no efforts in making domestic friendships. Interestingly, when asked if domestic students should make the first attempt for fostering friendships, many respondents (47\%) opted for neutral option. For the rest, opinion was divided (20\% disagreed, $33 \%$ agreed), suggesting a state of confusion on how to approach this matter. In view of this confusion there is perhaps a scope for arguing with other studies that international students be provided some guidance and opportunities to develop such friendships (Campbell, 2012; Poyrazli \& Kavanaugh, 2006; Ramsey et al., 2007). Such support is essential particularly when individual efforts are bearing no fruit.

On the other hand, there was some evidence that having friendships with local students linked to better adjustment. This was evident from the interviews, where students expressed that the domestic friendships helped in social adjustments. However, among those who claimed having domestic friends, some also noted that the nature of their friendship or bonding with host nationals was not as strong as they experienced in India. Nevertheless, seven interviewees said that they had benefited in the academic and social life contexts due to domestic friendships. 


\subsection{The role of information}

The final aspect that needs to be viewed in this section is the role played by provision of information in the adjustment process of international students. Some Indian students reported a few challenges in this aspect, particularly, with a lack of information. The findings of the study indicated the existence of differences in academic cultures between the educational systems of India and New Zealand. For example, when the respondents of the survey were asked to note if the academic work in New Zealand was different from that in India, a majority of the participants of the survey $(85 \%)$ reported that it was different. Likewise, a majority of respondents indicated that the teaching practices (89\%) and assessment procedures $(86 \%)$ were different too.

These variances brought challenges in the areas of their learning approaches, assessment procedures, fulfilling expectations of teachers, and designing their courses according to the stipulated norms. For example, when the participants of the survey were asked about thinking critically (Q. 27), a third responded that they found it slightly difficult and $24 \%$ indicated they found critical thinking moderately, very or extremely difficult, but $40 \%$ reported having no difficulty with critical thinking. Similarly, a doctoral student interviewee stated that the learning experiences in New Zealand were different and shared his experience with the concept of independent learning. Based on his perception he commented that supervisors in India closely monitored and guided the research students, while in New Zealand even though guidance existed, it was mostly independent learning, and guidance by the supervisors would be provided to a certain extent. A few other interviewees reported that they were struggling with their academic work as they were unsure of the expectations of their supervisors, and wished information regarding this be made available at an early stage of their studies. All these observations confirm the findings of other studies, which reported that international students inevitably encounter challenges in a different academic culture (Andrade, 2006b; Campbell \& Li, 2008; Church, 1982; Lewthwaite, 1996; Mehdizadeh \& Scott, 2005). Therefore, these studies insisted the need for providing appropriate information to the international students in order to better deal with their academic issues.

Having experienced these academic variances and consequent challenges, when Indian international students were asked if they were satisfied with their academic progress (Q. 33), 
remarkably an overwhelming majority of students (98\%) reported that they were satisfied with their progress. Even in terms of academic achievement (Q. 32), a majority of students (77\%) were found to be satisfied with their performances, while a few $(23 \%)$ reported average achievement. This level of satisfaction with academic performance is in sharp contrast with other studies which reported academic achievement was hampered by a lack of provision of information (Andrade, 2006b; Campbell \& Li, 2008; Mehdizadeh \& Scott, 2005).

In this context, one issue that needs to be raised is the struggle of Indian international students in acquiring information. The findings of the study indicated that Indian international students needed 'oral guidance' on the availability of the support services. They reported lack of information even though it was available online through university websites. For example, while $(16 \%)$ of doctoral/thesis in the survey reported dissatisfaction over the lack of provision of scholarship information, $24 \%$ of them stated that they were slightly satisfied. This view was also reiterated when the respondents were asked in a separate question to make comments on any other causes of their dissatisfaction. However, most universities supplied relevant information on scholarships or other funding opportunities through their websites. This suggests that even though information is provided, it is not accessed properly by Indian international students because the information is available on an unfamiliar platform. This finding is similar to the findings of Campbell and Li (2008) who showed that other Asian students also reported similar difficulties. Therefore, these experiences of students indicate the need of providing information to these students by using their familiar modes until they become used to the host country culture.

In this context, it may be argued that the responsibility of accessing information lies with the students. A finding of this study may be an appropriate example to illustrate this. A participant of the interview reported that his previous international exposure contributed to checking information online, while another student who had no previous international exposure reported to be struggling because information was available in an unfamiliar platform such as web resources via internet. Thus, the findings suggest that computer/internet literacy can vary between students, and a lack individual ability to access information may play a major role in affecting positive and faster adjustment to the conditions. At the same time, this observation cautions us not to entirely leave access to information to the individual's ability. Hence, it 
affirms the need for providing information by using an approach familiar to international students.

Further, the voice of the participants of this study suggested the areas in which they required information from their universities. These are: academic cultural variances, assessment procedures, academic activities, flexibility options in choosing courses. They anticipated effective orientation programmes with clear information on academic expectations and different educational approaches in New Zealand. In addition, other suggestions made by students interviewed including guidance and support for improving their writing skills. A small number requested information to be provided continually through supervisors/lecturers/tutors and through 'buddy programme' mentors. Such requests are in agreement with the recommendation of past studies which urged the involvement of teachers and host institutions in providing appropriate information to international students (Berno \& Ward, 2004; Campbell \& Li, 2008; Johnson, 2008; Lewthwaite, 1996; McClure, 2007; Mehdizadeh \& Scott, 2005; Soosay, 2009). The researcher can see the merit in such suggestions for addressing challenges described in the interviews.

Besides making suggestions to the educational institutions, the participants of the current study also made suggestions for future Indian international students to seek information prior to departure from the home country. As much as it is important to have post-entry information, the participants also assert that pre-entry information is vital for a better adjustment as it minimises adverse experiences. In fact, Indian students while reiterating the significant role played by the educational agents in India, also alerted to the lack of provision of information (informing about the existing conditions and academic expectations) for international students at a pre-entry stage. The opinions regarding provision of information at pre-entry as well as post-entry stages resonate with the literature review, which also draws attention to its benefit to international students (Andrade, 2006a; Arambewela \& Maringe, 2010; Berno \& Ward, 2004; Bethel \& Ward, 2014; Lewthwaite, 1996; McClure, 2007). Moreover, the findings of the current study are in line with the observations of studies which indicated that pre-entry stage information through agents (Bandyopadhyay, 2010), as well as post-entry information through support services (Pang, 2012) addressing an individual's concerns would be helpful. 
In addition, previous studies indicated that lack of information resulted in international students lacking in awareness of the available services and consequent underutilization of such support services (Ang \& Liamputtong, 2008; McKinlay et al., 1996; Sawir et al., 2009a, 2009b). In fact, some studies argued that educational institutions should take an active role in making sure that information was given to these students, for a better utilization of support services and for better adjustment to the conditions (Andrade, 2006a; Ang \& Liamputtong, 2008; Chun \& Poole, 2009; McKinlay et al., 1996; Ward, 2001; Zhang \& Goodson, 2011). Upholding these views, the findings of this study indicated the need for providing information through a familiar approach so as to enable international students to become aware of and utilise the available services. If these students could make use of the services from their early days of their stay, they would easily avoid the initial struggling experiences of their adjustment process.

\subsection{A model advised for better adjustment of international students}

Based on the findings of this study and the best practices identified in the related literature regarding the international students, the thesis proposes a model programme that can facilitate a better adjustment of international students. The suggested activities in the programme may be applicable to the whole international student community, irrespective of place and level of study. But, prior to proposing a model, two things will have to be acknowledged. Firstly, relevant language proficiency is a necessary aspect for entering higher education in an English speaking country, hence, international students are required to prove their individual abilities through language tests. Although help could be provided for the struggling students to cope with language difficulties, it is also vital to note that language proficiency also depends on individual abilities. Apart from this, the three key elements that can be applied simultaneously for the positive adjustment of international students in a host country are: the provision of effective support services in the educational institutions, developing friendships with domestic students, and a provision of information to the international students. Secondly, even though most of the contemporary literature held educational institutions responsible for caring for international students, this current study demonstrates individual abilities playing a key role. Therefore, this model assigns responsibility to both the international students and the educational institutions equally for better adjustment of international students. Moreover, the 
listed necessary activities are to be operated in the pre-entry as well as post-entry stages by both of them.

In brief, the activities to be performed by the international students are: making use of available support services provided for them by the educational institutions; making friendships with the host nationals; and accessing information and acquiring knowledge of the language usage styles, cultural aspects and academic expectations of the host country. Similarly, from the perspective of educational institutions, they are: facilitating international students with adequate support and trainings through workshops to develop the skills required; designing and organising programs that can improve the contacts between international and domestic students; and providing adequate information through approachable channels on the available resources and academic expectations of the educational system of the host country. All these activities in combination are expected to help international students' positive and faster adjustment to the conditions of the host country. Moreover, they would minimise their adjustment challenges related to the aspects of language, learning and living circumstances in the host country. To provide further clarity in this matter, the key features of the proposed activities are presented in a tabular form as shown in the table 7.1 below:

Table 7.1 - Activities proposed for prospective international students and educational institutions of a host country

\begin{tabular}{|c|c|c|c|c|}
\hline & \multicolumn{2}{|c|}{ International students } & \multicolumn{2}{|c|}{$\begin{array}{l}\text { Educational institutions of the host } \\
\text { country }\end{array}$} \\
\hline & Pre-entry & Post-entry & Pre-entry & Post-entry \\
\hline $\begin{array}{l}\text { Provision of } \\
\text { Support } \\
\text { services }\end{array}$ & $\begin{array}{l}\text { Approaching } \\
\text { educational } \\
\text { agents for } \\
\text { person to } \\
\text { person } \\
\text { discussion for } \\
\text { information or } \\
\text { for seeking any } \\
\text { assistance if } \\
\text { required }\end{array}$ & $\begin{array}{l}\text { Using } \\
\text { available } \\
\text { resources in } \\
\text { their } \\
\text { educational } \\
\text { institutions }\end{array}$ & $\begin{array}{l}\text { Providing realistic } \\
\text { information } \\
\text { through } \\
\text { educational } \\
\text { agents/ } \\
\text { representatives of } \\
\text { the universities/ } \\
\text { organising } \\
\text { international } \\
\text { education fairs }\end{array}$ & $\begin{array}{l}\text { Helping } \\
\text { international } \\
\text { students by } \\
\text { organising } \\
\text { trainings and } \\
\text { workshops to } \\
\text { improve their } \\
\text { knowledge and } \\
\text { skills to meet the } \\
\text { requirements }\end{array}$ \\
\hline
\end{tabular}




\begin{tabular}{|c|c|c|c|c|}
\hline $\begin{array}{l}\text { Developing } \\
\text { Friendships }\end{array}$ & $\begin{array}{l}\text { Developing } \\
\text { attitude towards } \\
\text { social } \\
\text { connectedness }\end{array}$ & $\begin{array}{l}\text { Making } \\
\text { friendships } \\
\text { with the host } \\
\text { nationals }\end{array}$ & $\begin{array}{l}\text { Designing } \\
\text { intercultural } \\
\text { promotional } \\
\text { programmes for } \\
\text { international and } \\
\text { domestic students }\end{array}$ & $\begin{array}{l}\text { Creating } \\
\text { opportunities for } \\
\text { developing } \\
\text { friendships } \\
\text { between } \\
\text { international and } \\
\text { domestic students }\end{array}$ \\
\hline $\begin{array}{l}\text { Provision of } \\
\text { Information }\end{array}$ & $\begin{array}{l}\text { Acquiring } \\
\text { knowledge } \\
\text { through web } \\
\text { resources } \\
\text { (Lang. usage, } \\
\text { cultural norms, } \\
\text { and academic } \\
\text { expectations) }\end{array}$ & $\begin{array}{l}\text { Acquiring } \\
\text { knowledge by } \\
\text { attending } \\
\text { orientations, } \\
\text { seminars, and } \\
\text { using web } \\
\text { resources }\end{array}$ & $\begin{array}{l}\text { Providing } \\
\text { information } \\
\text { through web } \\
\text { resources on the } \\
\text { realistic } \\
\text { conditions of the } \\
\text { host country, } \\
\text { culture, language } \\
\text { requirements and } \\
\text { academic } \\
\text { expectations }\end{array}$ & $\begin{array}{l}\text { Organizing } \\
\text { orientation } \\
\text { programmes and } \\
\text { seminars on the } \\
\text { availability of } \\
\text { facilities and } \\
\text { services, and on } \\
\text { the academic } \\
\text { expectations }\end{array}$ \\
\hline
\end{tabular}

\section{Description of the model:}

Based on the proposed activities for the benefit of international students shown in table 7.1 above, the following model (Figure 7.1) is developed. The analysis of the current study and recommendations of the related literature suggest that in general, international students encounter three types of challenges when they enter a new host country. These are: language, cross-cultural, and academic challenges. All these challenges will have impacts on the psychological and socio-cultural adjustment of individual international students. However, for achieving academic success in the new environment, international students need to take initiative to make positive psychological and socio-cultural adjustment to the conditions of the host country. When an individual has necessary knowledge and initiative, it would help to adjust to the conditions positively. Similar to this, the effective institutional support when provided, would help an individual to adapt better psychologically, and socio-culturally, and at the same time, a lack of effective support may lead to difficulties in both the adjustment processes. Other than these, both psychological and socio-cultural adjustment processes will have impacts on each other in a proportional manner. 
In the process of adjustment, both prospective international students and support services of educational institutions of the host country need to perform different activities simultaneously at pre-entry and post-entry stages. When these different activities are efficiently tackled by the stakeholders, it would help international students to acquire knowledge and skills to handle their inevitable challenges in the cross-cultural contexts. These skills may be categorised into language usage skills, knowledge about the cultural aspects of the host community, and knowledge of different academic culture of the host country. Ultimately, these different skills and knowledge would help international students to adapt positively to the conditions of the host country in terms of psychological, socio-cultural and academic aspects. Moreover, these positive impacts would result in achieving their academic goals successfully. The organization of different components of the proposed model are shown in the figure 7.1 below. For easy comprehension of the readers, the model is split into two parts, each specifying the activities of international students and educational institutions respectively in the following figures 7.2 and 7.3. 


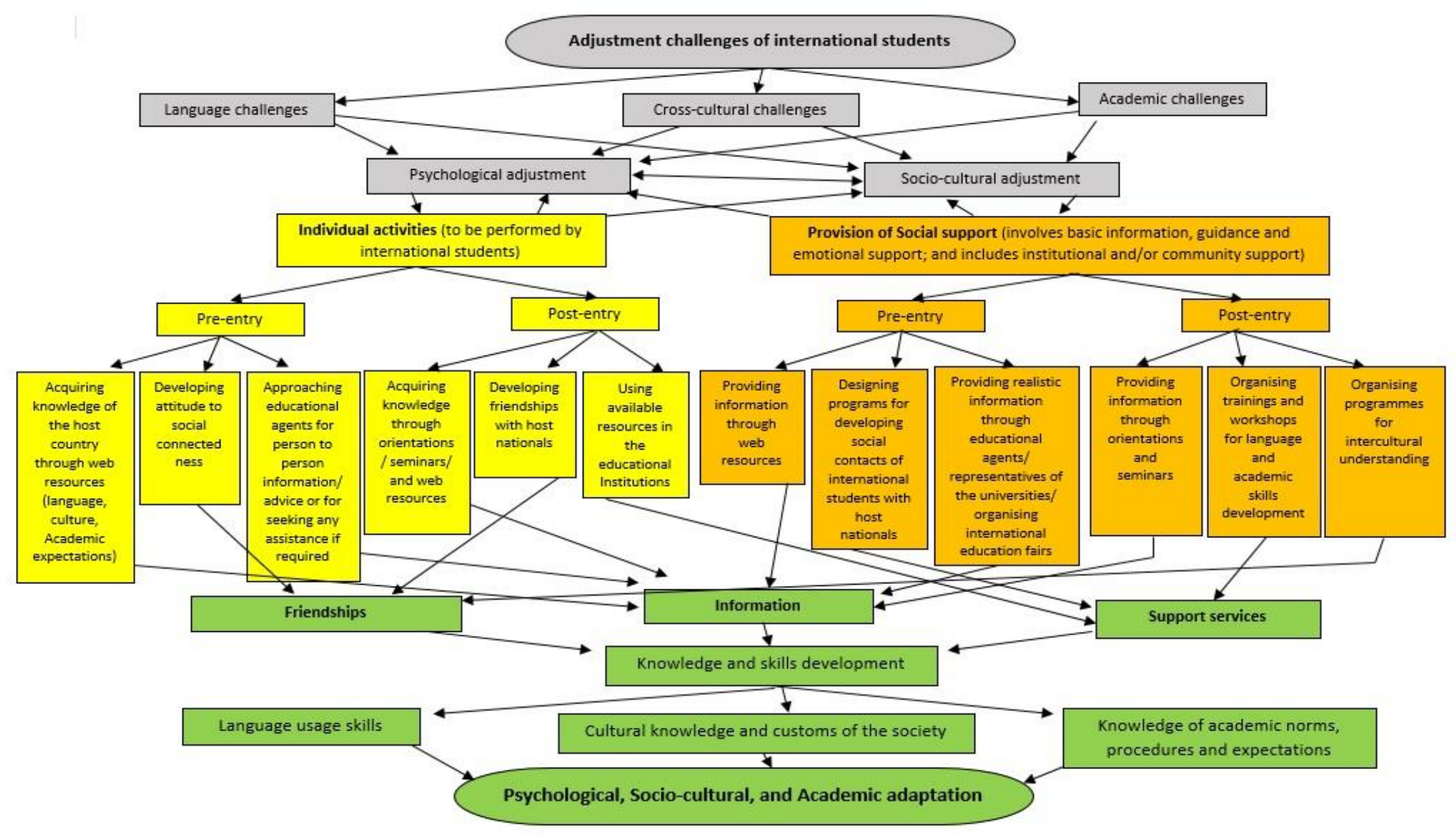

Figure 7.1 - A model for faster adjustment and academic success of international students 


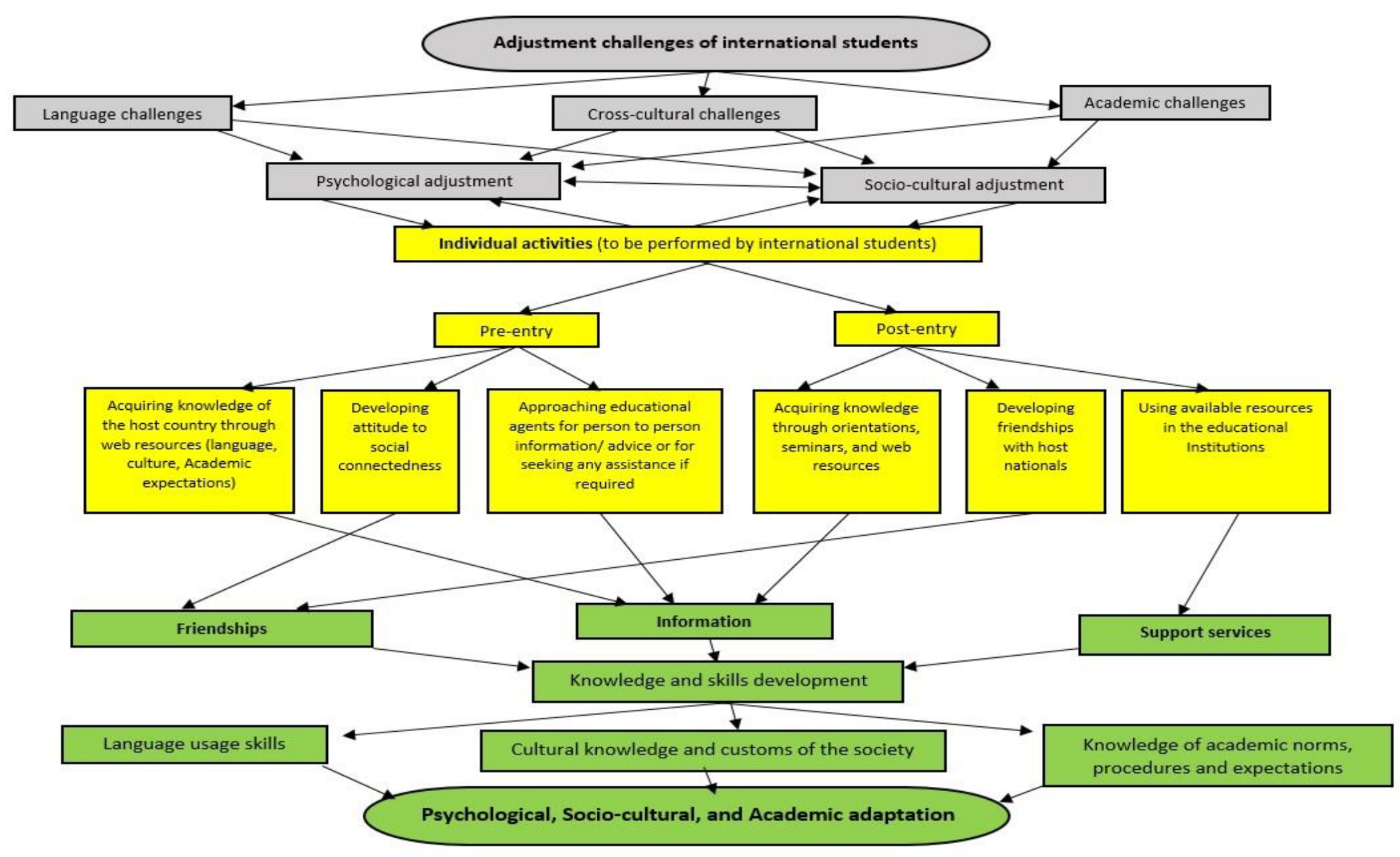

Figure 7.2 - Activities to be performed by the international students 


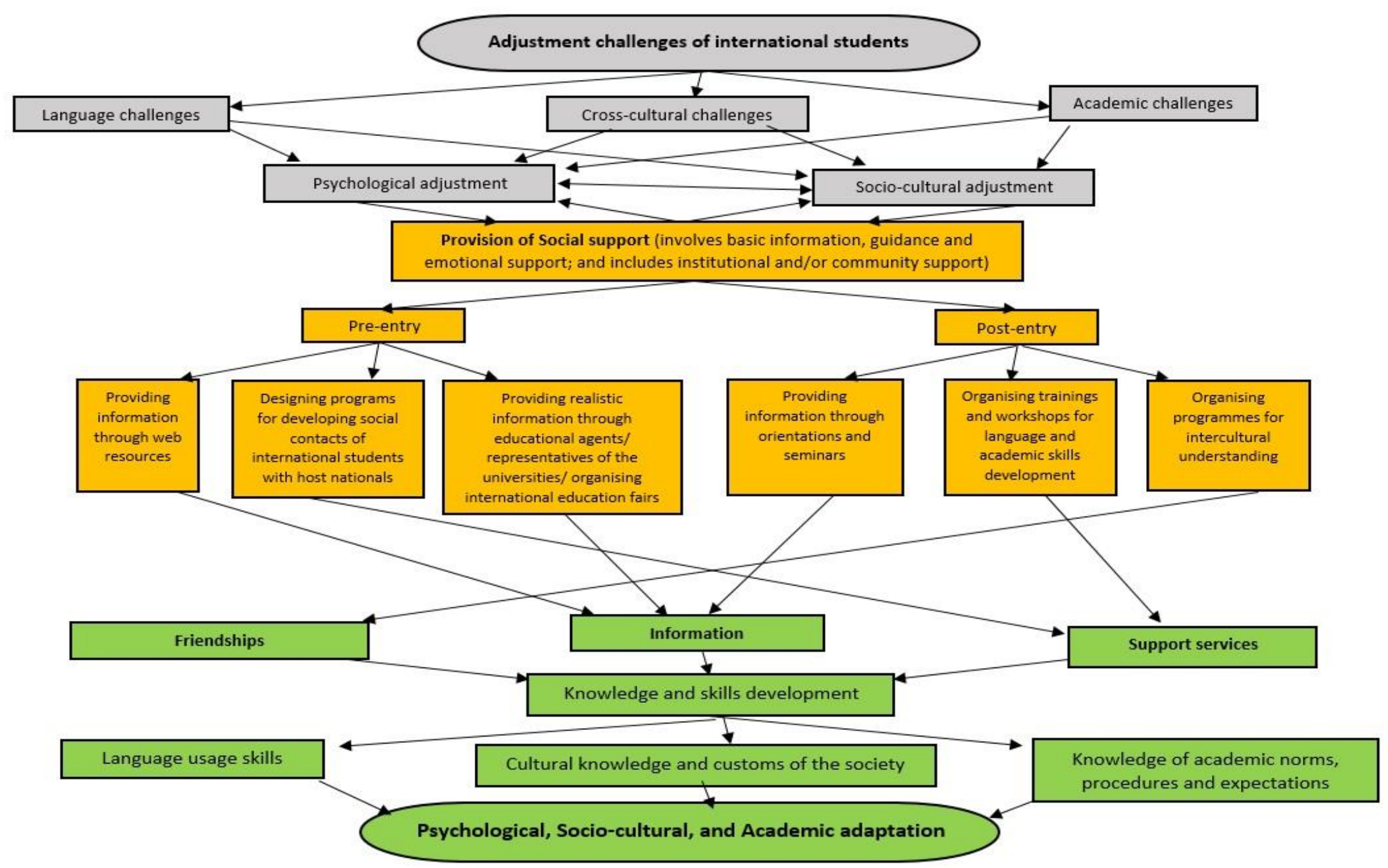

Figure 7.3 - Activities of institutions in providing social support to international students 


\section{The core of the proposal}

The suggested model specifies two significant issues that can be considered for a positive adjustment of international students in the host country. International students should make use of available support from their educational institutions intended for them, recognize the need for making friendships with host nationals and develop friendships with them, and should take initiative to access information and acquire necessary knowledge and skills helpful to their better adjustment to the conditions of the host country. Similarly, support services of educational institutions should provide appropriate support and equip international students with necessary skills by organising trainings and workshops, design and organise programmes that can improve the contacts between international and domestic students, and provide adequate information to these students on the available resources and academic expectations.

\subsection{Summary}

In this chapter, based on the observations of the experiences and challenges of Indian international students, interpretations were made on the significant findings related to their adjustment in New Zealand. These interpretations were mainly lensed through the recognised adjustment components (SLFI) as mentioned in the earlier discussion. Some of the observations were in agreement with studies referred to in this study, and some differed from the arguments of the past studies. Whether affirmed or differed, in the case of Indian international students, the following key observations are to be made. These students were found to be with adequate levels of English language proficiency which contributed to a better adjustment in New Zealand, whereas literature indicated that international students particularly from the Asian region in general encountered academic difficulties due to poor English language skills.

Furthermore, the participants reported that initially they were affected by the cultural differences between India and New Zealand in both academic and non-academic contexts. At the same time a majority of the participants maintained that their adjustment difficulties were limited to a few days to weeks of their initial period of stay in New Zealand. Despite experiencing differences in academic cultures between India and New Zealand, a better academic achievement with high level of satisfaction of the participants is reported in this 
study. Moreover, participants appreciated the new approaches to learning and assessment procedures of New Zealand education system. With regard to this, many other studies on Asian international students indicated that these differences in academic cultures severely impaired the academic progress of the participants in their studies.

Although most were able to handle challenges without significant distress, a few Indian students reported challenges with loneliness, low interactions with domestic students, and unfamiliar cultural contexts that were difficult to cope with and required specialist help. Many of the participants reported using web resources and maintaining frequent contacts with their friends and families in India and elsewhere. Thus, these frequent connections with their friends and families, by making use of the communication technology, seem to have helped them in getting emotional or advisory support whenever they encountered any social life challenges. In contrast, many past studies on Asian international students identified severe impacts of these challenges on the participants and recommended increased supporting measures in their institutions.

Generally, the related literature often indicated the responsibility of the educational institutions in making necessary provisions to support these international students. However, this study having noticed the expectations and experiences of international students, assigned the responsibility of adjustment to both individuals (i.e., international students) and the educational institutions. Therefore, based on the above observation, this study proposed an adjustment model with different activities which need to be addressed by both individual international students and educational institutions, to facilitate better adjustment of the international students in a new environment. Furthermore, these proposed activities are shown in a tabular and pictorial model to highlight various steps involved in the programme. The next chapter will present a brief summary of the findings of this study along with a note of strengths and limitations. 


\section{Chapter 8 \\ Conclusion}

\subsection{Introduction}

This chapter will provide a brief summary of the findings of this study. Subsequently, a note on the strengths and limitations of this study will be presented. Besides these, a few suggestions offered by the participants of this study for the betterment of conditions will be described.

\subsection{Purpose of the study}

The study had two objectives. The review of literature exposed specific gaps in the existing studies on international students' experiences in New Zealand, particularly related to the Indian international students. This indicated a need to explore the experiences and perspectives of these students adjusting to the conditions of academic and social lives in New Zealand. Apart from that, this study was also intended to find out the extent to which they have used the support services in their universities, and what they thought about such services, as they were meant to provide external support to international students in New Zealand.

\subsection{Summary of findings}

This was a mixed methods study, with data derived from two sources, a survey of Indian International Students $(\mathrm{N}=109)$ providing mainly quantitative data, and semi-structured interviews with 15 students providing qualitative data. With regard to the choice of a study destination, it was revealed that three factors almost equally influenced their decision to choose New Zealand. Firstly, like many other international students, the perception of good faculty and courses and quality of education was a major attraction for the Indian students. But it was not the only reason why they came to New Zealand. Peaceful and safe environment of the country was the second important factor that influenced their choice of New Zealand. In 
addition to that, financial considerations played role in encouraging some of them to study in New Zealand. These considerations included either an award of a scholarship or low course fees for their studies. Other than this, these students were also influenced by factors like personal interests, friends and family members living in New Zealand. With a few exceptions the students only considered studying in English speaking countries. However, although these Indian students presumably chose New Zealand because it was an English speaking country, it was not acknowledged as an important factor influencing their decision.

These findings revealed no significant language challenges faced by majority of these students either in academic or non-academic contexts. The issues mentioned were at a minor level with those of using colloquial expressions in daily life conversations, a different accent, and use of local style of humour. However, these challenges were limited to initial days or weeks until they became familiar with those aspects. This situation distinguishes the Indian students from the observations of past studies, where Asian students were generally identified with language difficulties. Unlike Asian international students in previous studies who have perceived their English language proficiency as a barrier to friendships with local students, these students were confident in their language proficiency. However, there was a perception among some (a little less than third of the respondents) that local students were not interested in friendships with local students.

Further, the findings informed that almost all the participants perceived the educational system in New Zealand to be different from what they experienced in India. As a result, a few experienced academic challenges such as issues with academic writing or giving oral presentations, but others adjusted to the new system, and some in fact enjoyed the new experience of writing and presenting. Cross-cultural differences also played a role for these students in accessing necessary information or in attaining guidance related to these academic issues. Nevertheless, despite these academic differences, a majority of the students reported higher levels of satisfaction with their learning experiences in New Zealand. It appears that adequate levels of English language proficiency of these students helped them in adapting to the academic requirements in the new country and to accomplish their academic tasks successfully. 
Our study revealed that a minority of students did not attend orientation programmes for several reasons, sometimes for reasons beyond their control, such as late arrival in the country due to delay in receiving visa. The failure in attending orientation programmes affected them adversely, as they missed necessary information. Besides this, some students had only departmental orientations which provided them with a limited amount of information. In those cases, when students failed to attend orientation programmes or had insufficient information, they faced challenging experiences in their academic and social life contexts. Further, lack of information made them unaware of the available support they could have in their universities, or of any specialised programmes intended for them, such as Buddy programmes, during their initial stages of stay. In most cases, students deployed their individual strategies, such as selfconfidence and determination, to overcome their academic or social life challenges. But based on their experiences, they expressed a desire for adequate support, most importantly to accomplish their academic tasks in a different educational setting.

Additionally, the findings demonstrated that a majority of Indian students were satisfied with their lives in New Zealand, as they maintained continuous contacts with their friends and families in India through social networking and web resources. Thus, these students could utilise the developments in communication technology effectively to acquire adequate emotional support. This new development seems to have completely changed the experiences of international students in a new and alien environment away from their homes. In an internet age it is necessary to rethink the concept of 'homesickness' or role of local friendship (see chapter 2.4.1), as these students always remain connected to their familiar networks. However, a few Indian students still experienced loneliness, isolation, and lamented the low interactions with domestic students. Consequently, they perceived that effective support provisions could help them to overcome these difficulties.

With regard to the accommodation facilities, above $90 \%$ of the students were found to be living in private accommodation arrangements. Some of them expressed their dissatisfaction with the expensive accommodation facilities provided by their universities. Although these students opted for private accommodation, the findings did not reveal any adverse experiences as indicated in some past studies. However, by opting for private accommodation they seemed to miss an opportunity to develop contacts with the domestic or other international students. The 
past studies reported that the accommodation facilities provided by the institutions could create opportunities for these students to become aware of different cultural aspects, as well as could keep them away from the impacts of isolation or loneliness.

Another important finding of this study was related to part-time work opportunities. A quarter of the respondents to the survey tried and could not find part-time work. Although most of them were eligible to work part-time, only a little over half (57\%) were able to secure a job; some of them (18\%) never even tried. Some of those who were unsuccessful in their efforts perceived that reasons such as their Indian name, poor impression of the employers about them, availability of limited part-time work opportunities, and difficulty in attaining references, influenced their chances of getting jobs. In a few cases, students reported that the discriminatory and non-cooperative attitudes of the staff of the support services (meant for this purpose) in their universities were responsible for their not getting a part-time work.

The study revealed that a majority of the Indian students in New Zealand 'never' or 'seldom' experienced any overt discrimination in their academic institutions, either from other students, or from academic and general staff. However, a few participants also reported some instances of perceived discrimination. They reported some cases of unfair treatment from their teachers, non-academic staff, domestic and other international students. But the majority of such instances of unfair treatment were from the wider community in New Zealand. The reported incidents were not of any serious nature of racial abuse or harassment, yet the students felt that they were being insulted on the ground of their ethnicity.

Regarding the services from the educational agents in India who represented their universities, a few students revealed that they had been provided with unreliable or misleading information by these agents, who concealed the realistic conditions of New Zealand. Further, they reported that this situation either made them upset when they faced the realities of life after their arrival in New Zealand, or led them to encounter challenges in their academic and non-academic contexts. These students expect that the activities of educational agents should be monitored by concerned universities. Students perceived that the consultants were mainly concerned with their profits, and less attentive to the welfare of the prospective students. 


\subsection{The significance of the findings}

In the absence of any major studies on Indian international students in New Zealand, this study will contribute to our understanding of the experiences of this particular student group. There are three significant findings of this study. First of all, it reveals that quality of education is only one of a number of other factors (such as safe environment and low fees) which draw Indian students to New Zealand. This perhaps explains a puzzle that we mentioned in Chapter 2 that although total number of Indian students coming to New Zealand is increasing every year, only a small proportion of them are coming to the universities. This indicates that in the perception of Indian students when they look for the destination of their higher studies, New Zealand still does not figure very highly in terms of the quality of education it offers particularly in comparison with other major destinations of global student mobility. And this implies that New Zealand universities will have to do more to establish the value of 'brand New Zealand' in the Indian education market.

The second significant finding of this study is that despite differences in academic cultures, a majority of Indian students at the end expressed high level of satisfaction with their academic experiences. It was noticed that adequate levels of their English language proficiency, added with their individual strategies, helped them to overcome their challenges in both academic and social life contexts. They could acquire help from different sources such as teachers, library staff, and classmates in dealing with their academic issues. Their English language proficiency certainly helped them to approach people whenever they required any help. Although, not all of them could develop friendships within the local host community, they were able to obtain emotional support from their friends and family members in India. They continued their contacts through social networking resources which helped them to recover from any psychological issues during the upsetting times. By all means, in dealing with the struggles of adjustment in a new environment, they have cleverly depended on their individual strategies, to minimise their challenges in both academic and social life contexts in New Zealand.

Thirdly, some participants of this study have revealed that they experienced challenges in both academic and non-academic contexts, particularly, during the initial periods of their stay in New Zealand. The reasons specified for this situation were their lack of awareness of the cross- 
cultural issues, or lack of information and guidance on the related matters. They particularly faced difficulties when they did not attend orientation programmes. Similarly, different educational conventions and procedures of New Zealand educational system also affected them adversely until they became familiar with those aspects. Moreover, in New Zealand, information related to different provisions and services of the universities is provided through orientation programmes, printed brochures, and web resources such as a university website. In New Zealand, domestic students are familiar with these different modes of information dissemination. However, some Indian students, as this study reveals, expected guidance through 'word of mouth'. They might have been accustomed to receive information or guidance in their culture in such a manner from their parents, friends and teachers in both academic and social life contexts. A few students mentioned that "It is not spoon-fed here"; it implied that they were spoon-fed in their own culture. A few participants highlighted the limited use of computers in India for educational purposes. While they had improved their computer skills after their arrival in New Zealand, but until and unless they became confident users of computers it was difficult for them to access any information through online sources. They also requested continuous support services from their academics, non-academic staff or through specially designed 'buddy' programmes, with a provision to approach them whenever they required any help. Although these are problems mentioned by a minority of students, this study recognises these cross-cultural issues impacting Indian students, particularly at the initial stages of their stay in New Zealand.

\subsection{Strengths and limitations of this study}

\section{Strengths}

The findings of this study have contributed new insights and perspectives on the Indian international students' experiences in their academic and social life in New Zealand. With reference to the existing literature on the subject so far, no studies are available exploring the experiences of Indian international students exclusively. Therefore, the outcomes of this study would be helpful in understanding their specific adjustment issues through their own perceptions and experiences. Consequently, the insights of the participants may help the 
educational providers in New Zealand to modify or introduce necessary strategies suitable to the cross cultural requirements of these students.

Secondly, the approach of mixed methods applied in this study has helped to obtain adequate amount of dual data which ultimately facilitated understanding of the research issues in an elaborate and in-depth manner. The initial survey data helped to identify experiences and adjustment issues at a broader level, and the interviews at a later stage helped to probe and extract in-depth information from the interviewees. Thus, this approach of mixed methods has given scope to the respondents to provide extensive data on their past and present experiences, and helped the researcher to better understand their experiences and expectations.

Thirdly, as the sampling of this study is from a homogeneous ethnic group of Indian international students, it helped us better understand their specific requirements in terms of cross-cultural aspects, which may not be generally possible with heterogeneous samples. This information will be helpful in formulating appropriate strategies for this specific group of students by the concerned institutions, to offer them better supporting services. The findings suggest, based on the generally high English language proficiency, and positive adjustment experiences of the respondents in this study, that India warrants greater attention from New Zealand tertiary institutions as a source country for international students.

Finally, this study shows that the Indian students had adequate levels of English language proficiency to meet the requirements of an English speaking environment. Many past studies highlighted that international students particularly from Asian region experienced greater levels of language difficulties. This study has shown that when individuals have adequate levels of relevant language proficiency, it enhances their confidence levels and helps them to achieve their primary goals, whether or not sufficient levels of external support is available.

\section{Limitations}

This study was confined to the experiences of Indian international students studying in New Zealand universities exclusively, and hence the findings and interpretations of this study may 
have limited applicability for all the international students studying in other sectors such as schools and polytechnics.

Similarly, as the findings of this study are related to a particular ethnic group of Indian international students, the findings may have restricted scope to be generalised for the heterogeneous international student community.

Likewise, the participants of this study come from heterogeneous backgrounds such as males/ females in different age groups, pursuing different levels of study in New Zealand universities, and coming from different linguistic and religious groups in India. Hence, there are risks in treating an individual experience or perception as applicable in general to all the international students.

Geographically, as this study has been conducted in New Zealand, the observations may not be applicable to the whole community of international students universally, which also may include Indian international students studying in other countries.

The issues related to the services of educational agents in India were not adequately dealt with in this study. Future research related to the international students may focus on this particular area.

\subsection{Implications for the universities and prospective students}

The findings of this study emphasise the following provisions to be made for the prospective international students by the universities in New Zealand. At the same time, the findings also indicated specific requirements for prospective international students considering opportunities for studying abroad.

\section{For the universities}

Information for international students should be channelled through multiple modes such as online sources, printed material and verbal instructions considering the cultural backgrounds 
of the international students. Equipping these students with necessary information and guidance will empower them to deal with their academic challenges even if the academic culture of New Zealand is different from those prevalent in their home country. Moreover, information at the initial stage of their studies would enable these students to make use of the available facilities and services in their institution from the early days of their stay. Thus, this provision would help them to enhance their knowledge and skills, and to meet the demands of the New Zealand education system.`

Availability of discounted accommodation (what is often provided by universities in other competing advanced countries) will be helpful to these students because of the multiple advantages associated with it. Importantly, this facility would provide them a safe and secured environment. Moreover, it would also create an opportunity to develop contacts with the domestic and other international students, which may enrich their intercultural understanding as well as to surmount academic or social life challenges, if any.

Creating more part time work opportunities within the universities may be considered to help these students with some financial support, as well as to give them some New Zealand work experience. Other than these benefits, this opportunity would also help them to extend their social networks, particularly with the host country community.

\section{For the prospective international students}

It is necessary for the prospective international students planning to study abroad to prepare psychologically to face any challenges that may arise in a new environment. For this, they need to acquire ample information and enhance their knowledge on the issues related to them. These issues may be associated with language aspects, learning approaches, culture and customs, lifestyles of the people, climatic conditions, or other related matters of the host country. Along with this information, they should also be aware of the strategies to be applied to overcome challenges if they encounter any.

It is also important for the prospective international students to develop an attitude of social connectedness to establish social contacts and friendships with the host nationals and other 
international students. It would be advantageous if they could make use of the accommodation facilities provided by the educational institutions, considering the multiple rewards associated with it. These advantages may be of an exposure to a multicultural environment, a safe and secured setting, and a place for creating opportunities to develop social contacts with the host nationals and other international students.

Most importantly, it is very essential to realise the importance of attending orientation programmes, seminars, and other specialised programmes intended for providing useful information to the international students in their educational institutions. These programmes would enlighten these students with necessary information and guidance on their learning and living conditions in the host country. At the same time, it is also important to explore and make use of the services available in their educational institutions to meet the requirements of their study and social life contexts. 


\section{References}

Acharya Nagarjuna University. (2012). Undergraduate course syllabus for BCA. Retrieved from http://www.nagarjunauniversity.ac.in/ugsyllabus/bcacourse.pdf

Acharya Nagarjuna University. (2014). Affiliated Colleges of the university. Retrieved from http://www.nagarjunauniversity.ac.in/collegedet.asp?id=16

Adler, P. S. (1975). The transitional experience: An alternative view of culture shock. Journal of Humanistic Psychology, 15(4), 13-23. doi: 10.1177/002216787501500403

Alazzi, K., \& Chiodo, J. J. (2006). Uncovering problems and identifying coping strategies of Middle Eastern university students. International Education, 35(2), 65-81.

Andrade, M. S. (2006a). A first-year seminar for International students. Journal of The FirstYear Experience \& Students in Transition, 18(1), 85-103.

Andrade, M. S. (2006b). International students in English-speaking universities: Adjustment factors. Journal of Research in International Education, 5(2), 131-154. doi: $10.1177 / 1475240906065589$

Andrade, M. S. (2009). The effects of English language proficiency on adjustment to university life. International Multilingual Research Journal, 3(1), 16 - 34.

Ang, P. L., \& Liamputtong, P. (2008). 'Out of the circle': International students and the use of university counselling services. Australian Journal of Adult Learning, 48(1), 108-130.

Arambewela, R., \& Maringe, F. (2010). International postgraduate students experience in a UK university: Lessons for academic practice. Research and Development in Higher Education: Reshaping Higher Education, 33, 29-38.

Araujo, A. A. d. (2011). Adjustment issues of international students enrolled in American colleges and universities: A review of the literature. Higher Education Studies, 1(1), 2 $-8$.

Baas, M. (2010). Imagined mobility: Migration and transnationalism among Indian students in Australia. London: Anthem Press.

Baker, T., Isaac, M., Li, M., \& Marshall, K. (2005). Learning expectations of different ethnic groups. New Zealand Journal of Applied Business Research, 4(1), 49-67.

Bandyopadhyay, S. (2013). India-New Zealand relations in the new century: A historical narrative of changing perceptions and shifting priorities. India quarterly(4), 317-333. 
Bandyopadhyay, S. (Ed.). (2009). Nationalist movement in India: A reader. Delhi; Oxford: Oxford University Press.

Bandyopadhyay, S. (Ed.). (2010). India in New Zealand: Local identities, global relations. Dunedin: Otago University Press.

Berno, T., \& Ward, C. (2004). Cross-cultural and educational adaptation of Asian students in New Zealand. Wellington: Asia 2000 Foundation.

Bethel, A., \& Ward, C. (2014). The information needs of international students in New Zealand tertiary institutions. Wellington, New Zealand: Immigration New Zealand \& Ministry of Business.

Bhandari, R., \& Blumenthal, P. (Eds.). (2010). International students and global mobility in higher education: National trends and new directions. International and Development Education. Palgrave Macmillan. 175 Fifth Avenue: New York, NY 10010. Web site: http://www.palgrave-usa.com; http://us.macmillan.com/internationalstudentsandglobalmobilityinhighereducation

Black, J. S., \& Mendenhall, M. (1991). The U-Curve adjustment hypothesis revisited: A review and theoretical framework. Journal of International Business Studies, 22(2), 225-247.

Black, J. S., Mendenhall, M., \& Oddou, G. (1991). Toward a comprehensive model of international adjustment: An integration of multiple theoretical perspectives. The Academy of Management Review, 16(2), 291-317.

Botti, M., \& Endacott, R. (2005). Clinical research 5: Quantitative data collection and analysis. Intensive \& Critical Care Nursing, 21(3), 187-193. doi: http://dx.doi.org/10.1016/j.iccn.2005.02.005

Brisset, C., Safdar, S., Lewis, J. R., \& Sabatier, C. (2010). Psychological and sociocultural adaptation of university students in France: The case of Vietnamese international students. International Journal of Intercultural Relations, 34(4), 413-426. doi: http://dx.doi.org/10.1016/j.ijintrel.2010.02.009

British Council. (2014). Postgraduate student mobility trends to 2024. London: British Council Press.

Brown, L. (2009). A failure of communication on the cross-cultural campus. Journal of Studies in International Education, 13(4), 439-454. doi: 10.1177/1028315309331913 
Brown, L., \& Holloway, I. (2008). The adjustment journey of international postgraduate students at an English university. Journal of Research in International Education, 7(2), 232-249. doi: 10.1177/1475240908091306

Business and Economic Research Limited. (2013). The satisfaction of international students in New Zealand universities and ITPS. Wellington, New Zealand: Ministry of Education.

Butcher, A. C., W., \& McGrath, T. (2004). International students in New Zealand: Needs and responses. International Education Journal, 5(4), 540-551.

Campbell, J., \& Li, M. (2008). Asian Students' voices: An empirical study of Asian students' learning experiences at a New Zealand university. Journal of Studies in International Education, 12(4), 375-396. doi: 10.1177/1028315307299422

Campbell, N. (2012). Promoting intercultural contact on campus. Journal of Studies in International Education, 16(3), 205-227. doi: 10.1177/1028315311403936

Campbell, N., \& Jieping, Z. (2006). Living in the west: A study of Chinese international students' adaptation. Communication Journal of New Zealand, 7, 1-31.

Cemalcilar, Z., \& Falbo, T. (2008). A longitudinal study of the adaptation of international students in the United States. Journal of Cross-Cultural Psychology, 39(6), 799-804. doi: $10.1177 / 0022022108323787$

Centre for Academic Mobility Research. (2013). Project Atlas: Trends and global data 2013. United States: Institute of International Education.

Chan, S.-J. (2012). Shifting patterns of student mobility in Asia. Higher Education Policy, 25(2), 207-224. doi: http://dx.doi.org/10.1057/hep.2012.3

Chen, C.-H., \& Zimitat, C. (2006). Understanding Taiwanese students' decision-making factors regarding Australian international higher education. The International Journal of Educational Management, 20(2), 91-100.

Choi, M. (1997). Korean students in Australian universities: Intercultural issues. Higher Education Research and Development, 16(3), 263 - 282.

Chun, J., \& Poole, D. L. (2009). Conceptualizing stress and coping strategies of Korean social work students in the United States: A concept mapping application. Journal of Teaching in Social Work, 29(1), 1 - 17.

Church, A. T. (1982). Sojourner adjustment. Psychological Bulletin, 91(3), 540-572. doi: 10.1037/0033-2909.91.3.540 
Cigularova, D. K. (2005). Psychosocial adjustment of international students. Colorado State University Journal of Student Affairs 14, 17-24.

Clark, N. (2006). Education in India. New York, USA: World Education Services.

Cortazzi, M., \& Jin, L. (1997). Communication for learning across cultures. In D. McNamara \& R. Harris (Eds.), Overseas students in higher education: Issues in teaching and learning (pp. 76-90). London: Routledge.

Creswell, J. W. (2003). Research design: Qualitative, quantitative, and mixed methods approaches. Thousand Oaks, CA: Sage.

Creswell, J. W. (2009). Research design: Qualitative, quantitative and mixed methods approaches (3rd ed.): Thousand Oaks, CA: Sage.

Creswell, J. W. (2010). When should I choose a mixed methods approach? Thousand Oaks, USA: Sage.

Creswell, J. W., \& Plano Clark, V. L. (2007). Designing and conducting mixed methods research. Thousand Oaks, CA: Sage.

Cubillo, J. M., Sánchez, J., \& Cerviño, J. (2006). International students' decision-making process. The International Journal of Educational Management, 20(2), 101-115.

De Wet, K. (2010). The importance of ethical appraisal in social science research: Reviewing a Faculty of Humanities' Research Ethics Committee. Journal of Academic Ethics, 8(4), 301-314. doi: http://dx.doi.org/10.1007/s10805-010-9118-8

Deloitte. (2008). The experiences of international students in New Zealand: Report on the results of the national survey 2007. Wellington, New Zealand: Ministry of Education.

Dolby, N., \& Rahman, A. (2008). Research in International Education. Review of Educational Research, 78(3), 676-726. doi: 10.3102/0034654308320291

Doyle, S., Gendall, P., Meyer, L. H., Hoek, J., Tait, C., McKenzie, L., \& Loorparg, A. (2010). An investigation of factors associated with student participation in study abroad. Journal of Studies in International Education, 14(5), 471-490. doi: $10.1177 / 1028315309336032$

Durkin, K. (2008). The middle way: East Asian Master's students' perceptions of critical argumentation in U.K. universities. Journal of Studies in International Education, 12(1), 38-55. doi: 10.1177/1028315307302839 
Duru, E., \& Poyrazli, S. (2007). Personality dimensions, psychosocial-demographic variables, and English language competency in predicting level of acculturative stress among Turkish international students. International Journal of Stress Management, 14(1), 99-110. doi: 0.1016/j.ijintrel.2004.08.005

Eaves, M. (2009). Learning styles technology and supporting overseas learners. Multicultural Education \& Technology Journal, 3(1), 61-73. doi: 10.1108/17504970910951156

Education New Zealand. (2015). Markets and Research: India. Retrieved from http://enz.govt.nz/markets-research/india

Furnham, A. (2004). Foreign students: Education and culture shock. Psychologist, 17, 16-19.

Gopinath, D. (2015). Characterizing Indian students pursuing global higher education: A conceptual framework of pathways to internationalization. Journal of Studies in International Education, 19(3), 283-305. doi: 10.1177/1028315314563783

Gray, D. E. (2004). Doing research in the real world. London; Thousand Oaks, CA: Sage.

Greene, J. C. (2007). Mixed methods in social inquiry (1st ed.). San Francisco, CA: JosseyBass.

Hall, B., \& Howard, K. (2008). A synergistic approach. Journal of Mixed Methods Research, 2(3), 248-269. doi: 10.1177/1558689808314622

Hanassab, S. (2006). Diversity, international students, and perceived discrimination: Implications for Educators and Counselors. Journal of Studies in International Education, 10(2), 157-172. doi: 10.1177/1028315305283051

Henderson, J. (2009). "It's all about give and take," Or is it?: Where, when and how do native and non-native uses of English shape U.K. university students' representations of each other and their learning experience? Journal of Studies in International Education, 13(3), 398-409. doi: 10.1177/1028315308329788

Ho, E., Holmes, P., \& Cooper, J. (2004). Review and evaluation of international literature on managing cultural diversity in the classroom. Wellington: Ministry of Education and Education New Zealand.

Ho, E. S., Li, W. W., Cooper, J., \& Holmes, P. (2007). The experiences of Chinese international students in New Zealand: The University of Waikato for the Ministry of Education, New Zealand.

Holmes, P. (2005). Ethnic Chinese students' communication with cultural others in a New Zealand university. Communication Education, 54(4), 289. 
Hopkins, P. (2007). Positionalities and knowledge: Negotiating ethics in practice. An International E-Journal for Critical Geographies, 6(3), 386-394.

Ivankova, N. V., Creswell, J. W., \& Stick, S. L. (2006). Using mixed-methods sequential explanatory design: From theory to practice. Field Methods, 18(1), 3-20. doi: $10.1177 / 1525822 \times 05282260$

Jackson, M., Ray, S. P., \& Bybell, D. (2013). International students in the U.S.: Social and psychological adjustment. Journal of International Students, 3(1), 17-28.

Johnson, B., \& Christensen, L. B. (2012). Educational Research: Quantitative, qualitative, and mixed approaches (4th ed.). Thousand Oaks, CA: Sage.

Johnson, B., \& Turner, L. A. (2003). Data collection strategies in Mixed methods research. In 11.Tashakkori \& Teddlie Handbook of mixed methods in social and behavioral research. (pp. 297-319). Thousand Oaks, CA: Sage.

Johnson, E. M. (2008). An investigation into pedagogical challenges facing international tertiary-level students in New Zealand. Higher Education Research \& Development, 27(3), $231-243$.

Johnson, H., \& Moloughney, B. (Eds.). (2006). Asia in the making of New Zealand. Auckland, N.Z.: Auckland University Press.

Johnson, R. B., Onwuegbuzie, A. J., \& Turner, L. A. (2007). Toward a definition of Mixed Methods research. Journal of Mixed Methods Research, 1(2), 112-133. doi: $10.1177 / 1558689806298224$

Junius, D. P. (1997). A study of the problems experienced by international students at Northern Arizona University. (Ed.D.), Northern Arizona University, United States -Arizona. Retrieved from http://proquest.umi.com/pqdweb?did=736836101\&Fmt=7\&clientId=7511\&RQT=309 $\underline{\& N \text { Name}}=\mathrm{PQD}$

Kaur, D. (2007). International students and American higher education: A study of the academic adjustment experiences of six Asian Indian international students at a Research Level II university. (Ed.D.), The University of North Carolina at Charlotte, United States -- North Carolina. Retrieved from http://proquest.umi.com/pqdweb?did=1212797381\&Fmt=7\&clientId=7511\&RQT $=30$ $\underline{9 \& \mathrm{VName}=\mathrm{PQD}}$ 
Khawaja, N. G., \& Dempsey, J. (2007). Psychological distress in international university students: An Australian study. Australian Journal of Guidance and Counselling, 17(1), 13-27.

King, R., Findlay, A., \& Ahrens, J. (2010). International student mobility literature review. London: Higher Education Funding Council for England.

Kumar, R. (2005). Research methodology: A step-by-step guide for beginners (2nd ed.). London: Sage.

Kvale, S., \& Brinkmann, S. (2009). Interviews: Learning the craft of qualitative research interviewing. Los Angeles: Sage.

Kwon, Y. (2009). Factors affecting international students' transition to higher education institutions in the United States: From the perspective of office of international students. College Student Journal, 43(4), 1020-1036.

Lee, J., \& Opio, T. (2011). Coming to America: Challenges and difficulties faced by African student athletes. Sport, Education and Society, 16(5), 629-644. doi: $10.1080 / 13573322.2011 .601144$

Lee, J. J. (2010). International students' experiences and attitudes at a US host institution: Self-reports and future recommendations. Journal of Research in International Education, 9(1), 66-84. doi: 10.1177/1475240909356382

Lee, J. J., \& Rice, C. (2007). Welcome to America? International student perceptions of discrimination. Higher Education, 53(3), 381-409. doi: 10.1007/s10734-005-4508-3

Leech, N. L., \& Onwuegbuzie, A. J. (2010). Guidelines for conducting and reporting mixed research in the field of counseling and beyond. Journal of Counseling and Development, 88(1), 61-70.

Lewis, N. (2005). Code of practice for the pastoral care of international students: Making a globalising industry in New Zealand. Globalisation, Societies and Education, 3(1), 5 47.

Lewthwaite, M. (1996). A study of international students' perspectives on cross-cultural adaptation. International Journal for the Advancement of Counselling, 19(2), 167185.

Li, G., Chen, W., \& Duanmu, J.-L. (2010). Determinants of international students' Academic performance. Journal of Studies in International Education, 14(4), 389-405. doi: $10.1177 / 1028315309331490$ 
Liu, J., McCreanor, T., McIntosh, T., \& Teaiwa, T. (Eds.). (2005). New Zealand identities: Departures and destinations. Wellington, N.Z.: Victoria University Press.

MacGregor, K. (2014). Global postgraduate student mobility trends to 2024. Retrieved from http://www.universityworldnews.com/article.php?story=20141009145454791

Malcolm, P., Ling, A., \& Sherry, C. (2004). Why do Chinese students study in New Zealand and how can they be helped to succeed? Paper presented at the annual international conference of the Higher Education Research and Development Society of Australasia (HERDSA), 4-7 July 2004, Miri, Sarawak. Retrieved from http://www.herdsa.org.au/wp-content/uploads/conference/2004/PDF/P018-jt.pdf

Mason, G. (2010). Violence against Indian students in Australia: A Question of dignity. Current Issues in Criminal Justice, 21(3), 461-466.

Mason, G. (2012). 'I am tomorrow': Violence against Indian students in Australia and political denial. Australian \& New Zealand Journal of Criminology, 45(1), 4-25. doi: $10.1177 / 0004865811431330$

McClure, J. W. (2007). International graduates' cross-cultural adjustment: Experiences, coping strategies, and suggested programmatic responses. Teaching in Higher Education, 12(2), 199 - 217.

McKinlay, N. J., Pattison, H. M., \& Gross, H. (1996). An exploratory investigation of the effects of a cultural orientation programme on the psychological well-being of international university students. Higher Education, 31(3), 379-395.

Mehdizadeh, N., \& Scott, G. (2005). Adjustment problems of Iranian international students in Scotland. International Education Journal, 6(4), 484-493.

Millar, G. (2009). Working with international students: Applied linguistics and the art of inclusive teaching. TESOL in Context S2 (Special Edition), 1-12.

Ministry of Education. (2001a). Export education in New Zealand: A Strategic approach to developing the sector. Wellington, New Zealand.

Ministry of Education. (2001b). Foreign fee - paying students in New Zealand: Trends. Wellington, New Zealand.

Ministry of Education. (2003). Code of practice for the pastoral care of international students (Revised 2003 ed.). Wellington, New Zealand.

Ministry of Education. (2007). Education strategy for India 2006-2009. Wellington, New Zealand. 
Ministry of Education. (2011a). International student enrolments in New Zealand 2004 2010 (pp. 1 - 17). Wellington, New Zealand.

Ministry of Education. (2011b). Prospects for international student enrolments in New

Zealand: Profiles of 13 source countries. Wellington: International Division, Ministry of Education, New Zealand.

Ministry of Education. (2012). New Zealand universities: Trends in international enrolments.

Wellington: International Division, Ministry of Education, New Zealand.

Ministry of Education. (2013). International student enrolments in New Zealand 2006-2012

(pp. 1-20). Wellington, New Zealand.

Mohd.Yusoff, Y. (2011). Self-Efficacy, perceived social support, and psychological adjustment in international undergraduate students in a public higher education institution in Malaysia. Journal of Studies in International Education. doi: $10.1177 / 1028315311408914$

Moser, S. (2008). Personality: A new positionality? Area, 40(3), 383-392. doi: 10.1111/j.1475-4762.2008.00815.x

mugging up. (2014). In thesaurus.com. Retrieved June 09, 2014, from http://thesaurus.com/browse/mugging+up

Muijs, D. (2011). Doing quantitative research in education with SPSS (2nd ed.). London ; Thousand Oaks: Sage.

Mumford, D. B. (1998). The measurement of culture shock. Soc Psychiatry Psychiatr Epidemiol(33), 149-154.

Nayak, R. R., \& Venkatraman, S. (2010). A pilot study into international students' academic culture: The context of Indian Business students in an Australian university. e-Journal of Business Education \& Scholarship of Teaching, 4(2), 1-12.

Novera, I. A. (2004). Indonesian postgraduate students studying in Australia: An examination of their academic, social and cultural experiences. International Education Journal, $5(4), 475-487$.

Oberg, K. (1960). Culture Shock: Adjustment to new cultural environments. Practical Anthropology, 7, 177-182.

OECD. (2013). Education indicators in focus, How is international student mobility shaping up? (pp. 1-4): OECD Publishing, Paris. 
OECD. (2014). How many students study abroad and where do they go? In Education at a Glance 2014: Highlights, (pp. 32-33): OECD Publishing, Paris.

Olivas, M., \& Li, C.-S. (2006). Understanding stressors of international students in higher education: What college counselors and personnel need to know. Journal of Instructional Psychology, 33(3), 217-222.

Onwuegbuzie, A. J., \& Collins, K. M. T. (2007). A typology of mixed methods sampling designs in social science research. Qualitative Report, 12(2), 281-316.

Onwuegbuzie, A. J., \& Johnson, R. B. (2006). The validity issue in Mixed research. Research in the Schools, 13(1), 48-63. doi: 10.1037/0003-066x.50.9.741

Onwuegbuzie, A. J., \& Leech, N. L. (2006). Linking research questions to mixed methods data analysis procedures. Qualitative Report, 11(3), 474-498.

Onwuegbuzie, A. J., Leech, N. L., \& Collins, K. M. T. (2010). Innovative data collection strategies in qualitative research. The Qualitative Report, 15(3), 696-726.

Onwuegbuzie, A. J., \& Teddlie, C. (2003). A framework for analysing data in Mixed methods research. In 13.Tashakkori \& Teddlie Handbook of mixed methods in social and behavioral research (pp. 351-383). Thousand Oaks, CA: Sage.

Pang, D. (2012). Enhancing academic learning advising in globally-engaged universities - A personal odyssey. Journal of the NUS Teaching Academy, 2(1), 24-41.

Plano Clark, V. L., \& Creswell, J. W. (2008). The mixed methods reader. Thousand Oaks, CA: Sage.

Poyrazli, S., \& Grahame, K. M. (2007). Barriers to adjustment: Needs of international students within a Semi-urban campus community. Journal of Instructional Psychology, 34(1), 28-45.

Poyrazli, S., \& Kavanaugh, P. R. (2006). Marital status, ethnicity, academic achievement, and adjustment strains: The case of graduate international students. College Student Journal. 40, from http://helicon.vuw.ac.nz/login?url=http://search.ebscohost.com/login.aspx?direct=true $\& \mathrm{db}=$ hlh $\& \mathrm{AN}=23588698 \&$ site $=$ ehost-live

Poyrazli, S., Kavanaugh, P. R., Baker, A., \& Al-Timimi, N. (2004). Social support and demographic correlates of acculturative stress in international students. Journal of College Counseling, 7(1), 73-82. doi: 10.1037/0022-3514.59.5.1006 
Poyrazli, S., \& Lopez, M. D. (2007). An exploratory study of perceived discrimination and homesickness: A comparison of international students and American students. The Journal of Psychology, 141(3), 263-280. doi: 10.3200/jrlp.141.3.263-280

Project Atlas. (2015). India's Students Overseas. Retrieved from http://www.iie.org/Services/Project-Atlas/India

Ramsey, P., Ramsey, D., \& Mason, R. (2007). The Massey Kiwi Friend Programme. Innovations in Education and Teaching International, 44(2), 109-118.

Rosenthal, D. A., Russell, J., \& Thomson, G. (2007). Social connectedness among international students at an Australian university. Social Indicators Research, 84(1), 71-82.

Rosenthal, D. A., Russell, J., \& Thomson, G. (2008). The health and wellbeing of international students at an Australian university. Higher Education, 55(1), 51-67. doi: 10.1007/s10734-006-9037-1

Rudestam, K. E., \& Newton, R. R. (2007). Surviving your dissertation: A comprehensive guide to content and process. Los Angeles: Sage.

Sandhu, D. S. (1994). An examination of the psychological needs of the international students: Implications for counselling and psythotherapy. International Journal for the Advancement of Counselling, 17(4), 229-239.

Sawir, E., Marginson, S., Deumert, A., Nyland, C., \& Ramia, G. (2008). Loneliness and international students: An Australian study. Journal of Studies in International Education, 12(2), 148-180. doi: 10.1177/1028315307299699

Sawir, E., Marginson, S., Nyland, C., Ramia, G., \& Rawlings-Sanaei, F. (2009a). The pastoral care of international students in New Zealand: Is it more than a consumer protection regime? Asia Pacific Journal of Education, 29(1), 45 - 59.

Sawir, E., Marginson, S., Nyland, C., Ramia, G., \& Rawlings-Sanaei, F. (2009b). The social and economic security of international students: A New Zealand study. Higher Education Policy, 22(4), 461-482.

Searle, W., \& Ward, C. (1990). The prediction of psychological and sociocultural adjustment during cross-cultural transitions. International Journal of Intercultural Relations, 14(4), 449-464. doi: 10.1016/0147-1767(90)90030-z

Soosay, C. (2009). International and domestic students' perspectives on teaching and learning. Journal of International Education in Business, 2(1), 20-32. 
Spencer-Oatey, H., \& Xiong, Z. (2006). Chinese students' psychological and sociocultural adjustments to Britain: An empirical study. Language, Culture and Curriculum, 19(1), $37-53$.

Statistics New Zealand. (2015). New Zealand in profile 2015. Wellington, New Zealand.

Sümer, S., Poyrazli, S., \& Grahame, K. (2008). Predictors of Depression and Anxiety among international students. Journal of Counseling and Development, 86(4), 429-437.

Swami, V. (2009). Predictors of sociocultural adjustment among sojourning Malaysian students in Britain. International Journal of Psychology, 44(4), 266 - 273.

Swami, V., Arteche, A., Chamorro-premuzic, T., \& Furnham, A. (2010). Sociocultural adjustment among sojourning Malaysian students in Britain: A replication and path analytic extension. Social Psychiatry and Psychiatric Epidemiology, 45(1), 57-65. doi: 10.1007/s00127-009-0042-4

Tarling, N. (2004). International students in New Zealand: The making of policy since 1950. Auckland, New Zealand: New Zealand Asia Institute, University of Auckland.

Tashakkori, A., \& Creswell, J. W. (2007a). Editorial: Exploring the nature of research questions in mixed methods research. Journal of Mixed Methods Research, 1(3), 207211. doi: $10.1177 / 1558689807302814$

Tashakkori, A., \& Creswell, J. W. (2007b). Editorial: The new era of mixed methods. Journal of Mixed Methods Research, 1(1), 3-7. doi: 10.1177/2345678906293042

Tashakkori, A., \& Teddlie, C. (2003). Handbook of mixed methods in social \& behavioral research. Thousand Oaks, CA: Sage.

Tashakkori, A., \& Teddlie, C. (2008). Quality of inferences in mixed methods research: Calling for an integrative framework. In M. M. Bergman (Ed.), Advances in mixed methods research: Theories and applications (pp. 101-119). London; Los Angeles: Sage.

Teddlie, C., \& Yu, F. (2007). Mixed methods sampling: A typology with examples. Journal of Mixed Methods Research, 1(1), 77-100. doi: 10.1177/2345678906292430

The University of Waikato. (2012). English language requirements for Graduate and Postgraduate study. Retrieved from http://www.waikato.ac.nz/students/international/entryreq/grad-postgrad-eng.shtml

Thurber, C. A., \& Walton, E. A. (2012). Homesickness and adjustment in university students. Journal of American College Health, 60(5), 415-419. doi:

$10.1080 / 07448481.2012 .673520$ 
Tochkov, K., Levine, L., \& Sanaka, A. (2010). Variation in the prediction of cross-cultural adjustment by Asian-Indian students in the United States. College Student Journal, 44(3), 677-689. doi: 10.1037/0022-0167.37.4.437.

Tremblay, K. (2005). Academic mobility and immigration. Journal of Studies in International Education, 9(3), 196-228. doi: 10.1177/1028315305277618

Turner, Y. (2009). "Knowing me, Knowing you," Is there nothing we can do?: Pedagogic challenges in using group work to create an intercultural learning space. Journal of Studies in International Education, 13(2), 240-255. doi: 10.1177/1028315308329789

United Nations-Department of Economic and Social Afairs-Population Division. (2015). World Population Prospects: The 2015 Revision, Key Findings and Advance Tables. (Working Paper No. ESA/P/WP.241 ed.). New York.

Universities New Zealand. (2012). Admission requirements for international students. Retrieved from http://www.universitiesnz.ac.nz/studying-in-nz/international

University of Pune. (2011). Syllabi. Retrieved from http://www.unipune.ac.in/university_files/syllabi.htm

Van Oudenhoven, J. P., \& Van der Zee, K. I. (2002). Predicting multicultural effectiveness of international students: The Multicultural Personality Questionnaire. International Journal of Intercultural Relations, 26(6), 679-694.

Verbik, L., \& Lasanowski, V. (2007). International student mobility: Patterns and trends. London: The Observatory on borderless higher education.

Vu, H., \& Doyle, S. (2014). Across borders and across cultures: Vietnamese students' positioning of teachers in a university twinning programme. Journal of Education for Teaching, 40(3), 267-283. doi: 10.1080/02607476.2014.903026

Wang, J. (2009). A study of resiliency characteristics in the adjustment of international graduate students at American universities. Journal of Studies in International Education, 13(1), 22-45. doi: 10.1177/1028315307308139

Wang, T., \& Shan, X. (2007). A qualitative study on Chinese postgraduate students' learning experiences in Australia. Paper presented at the AARE 2006 International education research conference : Adelaide : papers collection. http://www.aare.edu.au/06pap/wan06121.pdf

Ward, C. (2001). The impact of international students on domestic students and host institutions. Wellington, New Zealand. 
Ward, C. (2006). International students: Interpersonal, Institutional and Community impacts (July, 2006 ed.): Centre for Applied Cross-cultural Research, Victoria University of Wellington, Wellington.

Ward, C., Bochner, S., \& Furnham, A. (2001). The psychology of culture shock Hove, East Sussex; Philadelphia, PA: Routledge.

Ward, C., \& Kennedy, A. (1993). Where's the "culture" in cross-cultural transition? Journal of Cross-Cultural Psychology, 24(2), 221-249. doi: 10.1177/0022022193242006

Ward, C., \& Kennedy, A. (1999). The measurement of sociocultural adaptation. International Journal of Intercultural Relations, 23(4), 659-677.

Ward, C., \& Kennedy, A. (2001). Coping with cross-cultural transition. Journal of CrossCultural Psychology, 32(5), 636-642. doi: 10.1177/0022022101032005007

Ward, C., Leong, C.-H., \& Low, M. (2004). Personality and sojourner adjustment. Journal of Cross-Cultural Psychology, 137-151. doi: 10.1177/0022022103260719 35:

Ward, C., \& Masgoret, A.-M. (2004). The experiences of international students in New Zealand (pp. 161). Wellington: Ministry of Education.

Ward, C., Okura, Y., Kennedy, A., \& Kojima, T. (1998). The U-Curve on trial: A longitudinal study of psychological and sociocultural adjustment during cross-cultural transition. International Journal of Intercultural Relations, 22(3), 277-291.

Ward, C., \& Rana-Deuba, A. (1999). Acculturation and adaptation revisited. Journal of Cross-Cultural Psychology, 30(4), 422-442. doi: 10.1177/0022022199030004003

Zapf, M. K. (1991). Cross-cultural transitions and wellness: Dealing with culture shock. International Journal for the Advancement of Counselling, 14, 105-119.

Zhang, J., \& Goodson, P. (2011). Predictors of international students' psychosocial adjustment to life in the United States: A systematic review. International Journal of Intercultural Relations, 35(2), 139-162.

Zhou, Y., Jindal-Snape, D., Topping, K., \& Todman, J. (2008). Theoretical models of culture shock and adaptation in international students in higher education. Studies in Higher Education, 33(1), 63 - 75.

Zhou, Y., \& Todman, J. (2009). Patterns of adaptation of Chinese postgraduate students in the United Kingdom. Journal of Studies in International Education, 13(4), 467-486. doi: $10.1177 / 1028315308317937$ 


\section{Appendices \\ Appendix 1 - Survey Questionnaire \\ A Study of the Adjustment Experiences of Indian International students in \\ New Zealand Universities \\ Section A: Background Information}

(Please tick or write against the following where appropriate)

1. Your age group:

$17-24$
$25-29$
$30-34$
$35-39$
$40-44$
$45-49$

50 and over

2. Gender:

Male.

Female

3. Place of Origin:

Country of Citizenship

Country of Birth

Which State in India

4. Which of the following best describes your family circumstances while living in New Zealand?

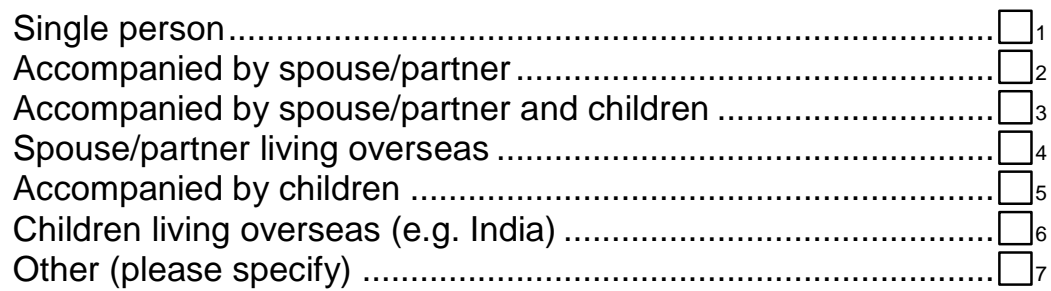

Additional comments if any:

5. Is this the first time you have studied outside of India?

Yes

No.

6. Is this the first time you have lived away from your family?

Yes.......

No. $\square_{2}$

Not applicable 
7. How long have you been in New Zealand?

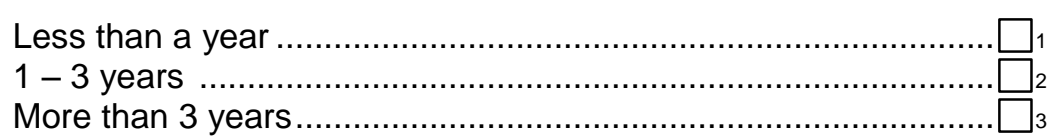

8. Which of the following best describes you?

Full fee-paying international student

Exchange Student

NZ International Doctoral Research Scholarship Student

International PhD Student (domestic status).

NZ International Undergraduate Fees Scholarship Student

Other scholarship / other programme (please specify)

9. What was your highest academic qualification before coming to New Zealand?

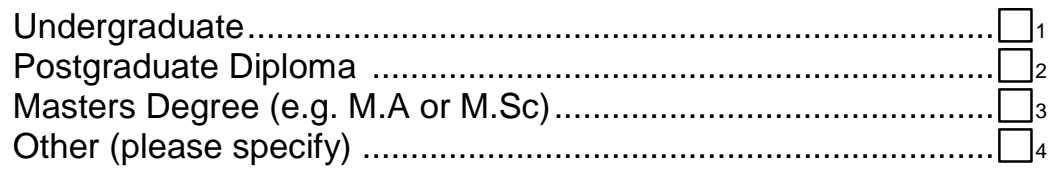

10. What were you doing in the year prior to enrolling at a New Zealand university?

Full-time student

Working as an employee

Individual business or self-employed

Other (please specify)

11. What course are you currently doing at your education institution?

Bachelor's degree.

Bachelor's degree (with Honours)

Graduate Certificate

Graduate Diploma

Postgraduate Certificate

Postgraduate Diploma

Master's degree

$\mathrm{PhD}$ degree......

Postdoctoral studies

Other (please specify)

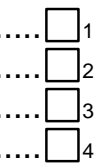

12. What is your main field of study?

Agriculture/Horticulture

Arts and Humanities

Creative arts and Design

Business/Management/Economics

Computing/Information Technology

Engineering

Medicine/Health Sciences

Social Sciences/Education

Other (please specify)

13. In which university are you studying now?

Auckland University of Technology

Lincoln University.

Massey University

University of Auckland 
University of Canterbury

University of Otago

University of Waikato

Victoria University of Wellington

14. Was New Zealand your first choice as a place of study?

Yes............ $\square_{1} \quad$ No ............ $\square_{2}$

15. If not, what was your first choice as a place of study?

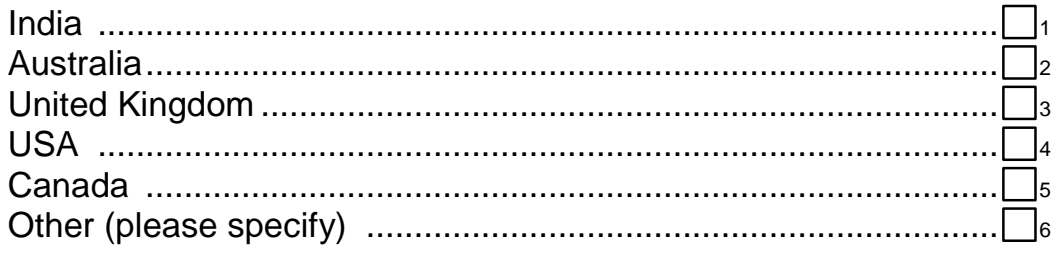

Additional comments if any:

16. Why did you choose New Zealand as your choice for a place of study?

17. What other education institution(s), if any, have you studied at in New Zealand? (Please tick all that apply)

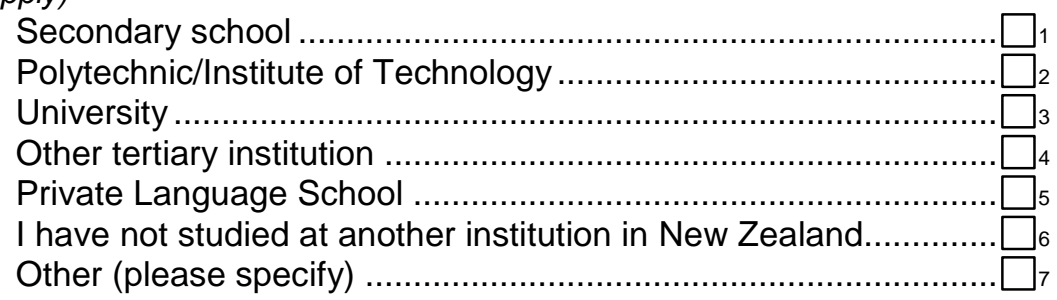

18. How financially difficult is it for you and / or your family, to pay for your education in New Zealand?

Not at all.

Slightly (a little)

Moderately

Very

Extremely.....

Don't Know / Not Sure 


\section{English Language proficiency}

19. Have you taken an IELTS or TOEFL test?

IELTS.

TOEFL...... $\square_{2}$

None......

(Please go to Q.21)

20. If you have taken an IELTS or TOEFL test, what was your most recent test score?

\begin{tabular}{|l|l|l|}
\hline \multicolumn{1}{|c|}{ Type of IELTS or TOEFL test } & \multicolumn{1}{c|}{ Test Score } & $\begin{array}{c}\text { Year test taken } \\
\text { e.g. 2009 }\end{array}$ \\
\hline a. IELTS score $(0-9)$ & a. & \\
\hline b. TOEFL (paper and pencil test) score $(310-677)$ & b. & \\
\hline c. TOEFL (computer test) score $(0-300)$ & c. & \\
\hline d. TOEFL (internet based) score $(0-120)$ & d. & \\
\hline
\end{tabular}

21. Did you go to an English medium educational institution in India?

Yes....

No.

22. Please indicate how often you spoke English in the following situations in India?

\begin{tabular}{|l|c|c|c|c|}
\hline & Never & Seldom & Sometimes & Often \\
\hline a. At home & $\square_{1}$ & $\square_{2}$ & $\square_{3}$ & $\square_{4}$ \\
\hline b. Among friends & $\square_{1}$ & $\square_{2}$ & $\square_{3}$ & $\square_{4}$ \\
\hline c. At work place & $\square_{1}$ & $\square_{2}$ & $\square_{3}$ & $\square_{4}$ \\
\hline
\end{tabular}

23. Thinking about your academic studies, how would you rate your English language ability in the following areas?

\begin{tabular}{|l|c|c|c|c|}
\hline & Poor & Average & Good & Excellent \\
\hline a. Reading & $\square_{1}$ & $\square_{2}$ & $\square_{3}$ & $\square_{4}$ \\
\hline b. Writing & $\square_{1}$ & $\square_{2}$ & $\square_{3}$ & $\square_{4}$ \\
\hline c. Listening & $\square_{1}$ & $\square_{2}$ & $\square_{3}$ & $\square_{4}$ \\
\hline d. Speaking & $\square_{1}$ & $\square_{2}$ & $\square_{3}$ & $\square_{4}$ \\
\hline e. Overall & $\square_{1}$ & $\square_{2}$ & $\square_{3}$ & $\square_{4}$ \\
\hline
\end{tabular}

24. Thinking about communication in everyday contexts (shopping, socialising, bureaucracy etc.), how would you rate your English language ability in the following areas?

\begin{tabular}{|l|c|c|c|c|}
\hline & Poor & Average & Good & Excellent \\
\hline a. Reading & $\square_{1}$ & $\square_{2}$ & $\square_{3}$ & $\square_{4}$ \\
\hline b. Writing & $\square_{1}$ & $\square_{2}$ & $\square_{3}$ & $\square_{4}$ \\
\hline c. Listening & $\square_{1}$ & $\square_{2}$ & $\square_{3}$ & $\square_{4}$ \\
\hline d. Speaking & $\square_{1}$ & $\square_{2}$ & $\square_{3}$ & $\square_{4}$ \\
\hline e. Overall & $\square_{1}$ & $\square_{2}$ & $\square_{3}$ & $\square_{4}$ \\
\hline
\end{tabular}

\section{Section B - Educational Experiences in New Zealand}

\section{Learning experiences}

25. How similar is your academic work in a New Zealand university compared to your academic work in an Indian educational institution? (Please tick one box for each item) 


\begin{tabular}{|l|c|c|c|c|c|}
\hline & Very Different & Different & Similar & Very similar & Unsure \\
\hline a. Academic workload & $\square_{1}$ & $\square_{2}$ & $\square_{3}$ & $\square_{4}$ & $\square_{5}$ \\
\hline b. Teaching practices & $\square_{1}$ & $\square_{2}$ & $\square_{3}$ & $\square_{4}$ & $\square_{5}$ \\
\hline c. Assessment procedures & $\square_{1}$ & $\square_{2}$ & $\square_{3}$ & $\square_{4}$ & $\square_{5}$ \\
\hline $\begin{array}{l}\text { d. Interaction between } \\
\text { students and lecturers }\end{array}$ & $\square_{1}$ & $\square_{2}$ & $\square_{3}$ & $\square_{4}$ & $\square_{5}$ \\
\hline $\begin{array}{l}\text { e. Interaction with other } \\
\text { students in your class }\end{array}$ & $\square_{1}$ & $\square_{2}$ & $\square_{3}$ & $\square_{4}$ & $\square_{5}$ \\
\hline
\end{tabular}

26. Overall, do you find the academic workload in New Zealand

Too little

About right

Too much.

27. How difficult are the following educational activities for you? (If the activity is not relevant to your course of study, tick "not applicable")

\begin{tabular}{|c|c|c|c|c|c|c|}
\hline & \multicolumn{6}{|c|}{ (Please tick one box only for each activity) } \\
\hline & $\begin{array}{c}\text { Not at } \\
\text { all } \\
\text { difficult }\end{array}$ & $\begin{array}{l}\text { Slightly } \\
\text { (a little) } \\
\text { difficult }\end{array}$ & $\begin{array}{l}\text { Moderately } \\
\text { difficult }\end{array}$ & $\begin{array}{c}\text { Very } \\
\text { difficult }\end{array}$ & $\begin{array}{l}\text { Extremely } \\
\text { difficult }\end{array}$ & $\begin{array}{c}\text { Not } \\
\text { Applicable }\end{array}$ \\
\hline a. Understanding teachers/lecturers & $\square_{1}$ & $\square_{2}$ & $\square_{3}$ & $\square_{4}$ & $\square_{5}$ & $\square_{6}$ \\
\hline b. Writing assignments & $\square_{1}$ & ]$_{2}$ & ]$_{3}$ & 4 & $7_{5}$ & 76 \\
\hline c. Taking notes during class & $\bar{Z}_{1}$ & 2 & ]$_{3}$ & 4 & ]$_{5}$ & ]$_{6}$ \\
\hline d. Completing assignments on time & $\square_{1}$ & ]$_{2}$ & ]$_{3}$ & ]$_{4}$ & ]$_{5}$ & ]$_{6}$ \\
\hline e. Working on group projects & $\square_{1}$ & ]$_{2}$ & ]$_{3}$ & $\overline{4}$ & ]$_{5}$ & 76 \\
\hline f. Taking tests or exams & $\square_{1}$ & 2 & 3 & 4 & ]$_{5}$ & ]$_{6}$ \\
\hline g. Making oral (spoken) presentations & $\square_{1}$ & ]$_{2}$ & ]$_{3}$ & $\sqrt{4}$ & 5 & $\square_{6}$ \\
\hline h. Managing your study workload & $\square_{1}$ & 2 & ]$_{3}$ & $\sqrt{4}$ & ]$_{5}$ & $\overline{6}$ \\
\hline i. Asking questions in class & ]$_{1}$ & ]$_{2}$ & ]$_{3}$ & 4 & ]$_{5}$ & $\sqrt{6}$ \\
\hline j. Thinking critically (analytically) & $\square_{1}$ & ]$_{2}$ & ]$_{3}$ & 4 & ]$_{5}$ & $\overline{6}$ \\
\hline $\begin{array}{l}\text { k. Expressing yourself } \\
\text { (communicating) in English }\end{array}$ & $\square_{1}$ & $\square_{2}$ & $\beth_{3}$ & $\square_{4}$ & $\beth_{5}$ & $\square 6$ \\
\hline $\begin{array}{l}\text { I. Expressing (Giving) your opinions to } \\
\text { your teacher/lecturer }\end{array}$ & $\square_{1}$ & ]$_{2}$ & $\beth_{3}$ & $\square_{4}$ & ]$_{5}$ & $\square_{6}$ \\
\hline $\begin{array}{l}\text { m. Studying in a different educational } \\
\text { system }\end{array}$ & $\square_{1}$ & $\square_{2}$ & $\square_{3}$ & $\square_{4}$ & $\square_{5}$ & $\square 6$ \\
\hline
\end{tabular}

28. Thinking about your overall experience with your programme of study/qualification, please rate the quality of the following.

\begin{tabular}{|l|c|c|c|c|c|}
\hline & Poor & Average & Good & Excellent & $\begin{array}{c}\text { Not } \\
\text { Applicable }\end{array}$ \\
\hline a. The content of your course(s) & $\square_{1}$ & $\square_{2}$ & $\square_{3}$ & $\square_{4}$ & $\square_{5}$ \\
\hline b. The feedback 7 you receive & $\square_{1}$ & $\square_{2}$ & $\square_{3}$ & $\square_{4}$ & $\square_{5}$ \\
\hline c. The quality of your lecturers & $\square_{1}$ & $\square_{2}$ & $\square_{3}$ & $\square_{4}$ & $\square_{5}$ \\
\hline d. The assessment procedures & $\square_{1}$ & $\square_{2}$ & $\square_{3}$ & $\square_{4}$ & $\square_{5}$ \\
\hline
\end{tabular}

29. The 3 things that you have enjoyed most about academic life in New Zealand are:

(i)

${ }^{7}$ Feedback - information you receive about how well you are doing in your studies. 
(ii)

(iii)

30. The 3 most difficult things that you have faced in terms of adjusting to academic work in New Zealand have been:

(i)

(ii)

(iii)

31. Please describe what (if anything) has helped you adjust to academic work in New Zealand? 


\section{Learning achievement}

32. How well are you doing in your studies in New Zealand? (Please tick one box only)

\begin{tabular}{|c|c|c|c|}
\hline Poor & Average & Good & Excellent \\
\hline$\square_{1}$ & $\square_{2}$ & $\square_{3}$ & $\square_{4}$ \\
\hline
\end{tabular}

33. How satisfied are you with your progress in your studies? (Please tick one box only)

\begin{tabular}{|c|c|c|c|c|}
\hline $\begin{array}{c}\text { Not at all } \\
\text { satisfied }\end{array}$ & $\begin{array}{c}\text { Slightly (a little) } \\
\text { satisfied }\end{array}$ & $\begin{array}{c}\text { Moderately } \\
\text { satisfied }\end{array}$ & $\begin{array}{c}\text { Very } \\
\text { satisfied }\end{array}$ & $\begin{array}{c}\text { Extremely } \\
\text { satisfied }\end{array}$ \\
\hline$\square_{1}$ & $\square_{2}$ & $\square_{3}$ & $\square_{4}$ & $\square_{5}$ \\
\hline
\end{tabular}

34. If you are not satisfied with your progress, please could you elaborate?

(Questions 35 and 36 are for Masters and PhD Thesis students only) (Others please continue to Qn.37)

35. Please rate your satisfaction with the following aspects of doctoral/thesis work.

\begin{tabular}{|c|c|c|c|c|c|c|}
\hline & $\begin{array}{l}\text { Not } \\
\text { applicabl } \\
\text { e }\end{array}$ & $\begin{array}{l}\text { Not at } \\
\text { all } \\
\text { satisfie } \\
\text { d }\end{array}$ & $\begin{array}{l}\text { Slightly } \\
\text { satisfie } \\
\text { d }\end{array}$ & $\begin{array}{l}\text { Moderatel } \\
\text { y satisfied }\end{array}$ & $\begin{array}{l}\text { Very } \\
\text { satisfie } \\
\quad \text { d }\end{array}$ & $\begin{array}{l}\text { Extremel } \\
y \\
\text { satisfied }\end{array}$ \\
\hline $\begin{array}{l}\text { a. Access to clear information on what is } \\
\text { required of you as a doctoral/thesis } \\
\text { student }\end{array}$ & $\square_{1}$ & $\square_{2}$ & $\beth_{3}$ & $\beth_{4}$ & ]$_{5}$ & ]$_{6}$ \\
\hline $\begin{array}{l}\text { b. Access to clear information on your } \\
\text { rights as a doctoral/thesis student }\end{array}$ & $\square_{1}$ & $\square_{2}$ & ]$_{3}$ & $\square_{4}$ & ]$_{5}$ & ]$_{6}$ \\
\hline $\begin{array}{l}\text { c. Opportunities for scholarly interactions } \\
\text { with other postgraduate students }\end{array}$ & $\square_{1}$ & $\beth_{2}$ & \rfloor$_{3}$ & ]$_{4}$ & ]$_{5}$ & ]$_{6}$ \\
\hline $\begin{array}{l}\text { d. Opportunities to interact with academic } \\
\text { staff other than your supervisor(s) }\end{array}$ & $\square_{1}$ & ]$_{2}$ & \rfloor$_{3}$ & ]$_{4}$ & ]$_{5}$ & ]$_{6}$ \\
\hline $\begin{array}{l}\text { e. Opportunities for skills development } \\
\text { e.g. Writing proposals }\end{array}$ & $\beth_{1}$ & $\square_{2}$ & \rfloor$_{3}$ & ]$_{4}$ & ]$_{5}$ & ]$_{6}$ \\
\hline $\begin{array}{l}\text { f. Opportunities to participate and present } \\
\text { at research seminars }\end{array}$ & $\square_{1}$ & $\square_{2}$ & ]$_{3}$ & ]$_{4}$ & ]$_{5}$ & ] 6 \\
\hline $\begin{array}{l}\text { g. The academic guidance provided by } \\
\text { your supervisor(s) }\end{array}$ & $\square_{1}$ & $\square_{2}$ & $\beth_{3}$ & $\square_{4}$ & \rfloor$_{5}$ & ]$_{6}$ \\
\hline $\begin{array}{l}\text { h. Information and support provided on } \\
\text { using Library resources }\end{array}$ & $\square_{1}$ & $\square_{2}$ & $\square_{3}$ & $\square_{4}$ & $\square_{5}$ & $\square_{6}$ \\
\hline $\begin{array}{l}\text { i. Information and support provided by } \\
\text { Information Technology Services }\end{array}$ & $\square_{1}$ & $\square_{2}$ & $\beth_{3}$ & $\beth_{4}$ & $\square_{5}$ & $\beth_{6}$ \\
\hline $\begin{array}{l}\text { j. Information provided on different } \\
\text { scholarships /Funding opportunities }\end{array}$ & $\square_{1}$ & $\square_{2}$ & $\beth_{3}$ & $\beth_{4}$ & $\beth_{5}$ & ]$_{6}$ \\
\hline
\end{tabular}

36. If you are not satisfied with aspects of your experiences as a post-graduate student, please elaborate? 


\section{Section C - Services and Facilities}

37. Please rate the quality of the services or facilities that are available in your institution.

(Please tick one box only for each service/facility)

\begin{tabular}{|c|c|c|c|c|c|c|}
\hline & Poor & Average & Good & Excellent & $\begin{array}{l}\text { Don't } \\
\text { know }\end{array}$ & $\begin{array}{c}\text { Not } \\
\text { Available }\end{array}$ \\
\hline a. International Student Office & $\square 1$ & $\square_{2}$ & $\square_{3}$ & $\square 4$ & $\square_{5}$ & $\square_{6}$ \\
\hline b. Student orientation services & $\beth_{1}$ & $\square_{2}$ & $\bar{\Pi}_{3}$ & $\bar{\Pi}_{4}$ & $\square_{5}$ & ]$_{6}$ \\
\hline c. Health services & $\beth_{1}$ & ]$_{2}$ & ]$_{3}$ & ]$_{4}$ & ]$_{5}$ & ]$_{6}$ \\
\hline d. Financial advice services & $\square_{1}$ & $\square_{2}$ & ]$_{3}$ & ]$_{4}$ & $\square_{5}$ & $\square_{6}$ \\
\hline e. Accommodation services & $\square 1$ & $\square_{2}$ & ]$_{3}$ & ]$_{4}$ & $\square_{5}$ & $\square 6$ \\
\hline f. Vocational (career) or educational guidance & $\square_{1}$ & $\bar{J}_{2}$ & $\bar{J}_{3}$ & $\bar{\Pi}_{4}$ & $\square_{5}$ & $\overline{7}_{6}$ \\
\hline g. Counselling services & $\square_{1}$ & $\square_{2}$ & ]$_{3}$ & ]$_{4}$ & $\square_{5}$ & ]$_{6}$ \\
\hline h. Computing services & $\square_{1}$ & ]$_{2}$ & ]$_{3}$ & $\bar{\square}_{4}$ & $\square_{5}$ & ]$_{6}$ \\
\hline i. Library services & $\square_{1}$ & $\beth_{2}$ & ]$_{3}$ & ]$_{4}$ & $\square_{5}$ & $\square_{6}$ \\
\hline j. Language laboratories & $\square_{1}$ & $\beth_{2}$ & ]$_{3}$ & ]$_{4}$ & $\square_{5}$ & $\square_{6}$ \\
\hline k. Learning Support services & $\square_{1}$ & $\square_{2}$ & $\square_{3}$ & ]$_{4}$ & $\square_{5}$ & $\square_{6}$ \\
\hline I. "Buddy" or mentor programmes & $\square_{1}$ & $\square_{2}$ & $\square_{3}$ & $\square_{4}$ & $\square_{5}$ & $\square_{6}$ \\
\hline m. Sports and Recreational facilities & $\square_{1}$ & $\beth_{2}$ & $\square_{3}$ & $\square_{4}$ & $\square_{5}$ & $\square_{6}$ \\
\hline n. Clubs and societies & $\square_{1}$ & $\eta_{2}$ & $\square_{3}$ & $\square_{4}$ & $\square_{5}$ & $\square 6$ \\
\hline o. Student Association & $\square_{1}$ & $\square_{2}$ & $\square_{3}$ & $\square_{4}$ & $\square_{5}$ & $\square 6$ \\
\hline p. Additional services & $\square_{1}$ & $\square_{2}$ & $\square_{3}$ & $\square_{4}$ & $\square_{5}$ & $\square_{6}$ \\
\hline
\end{tabular}

38. How much use do you make of different services and facilities available in your university?

\begin{tabular}{|c|c|c|c|c|}
\hline $\begin{array}{c}\text { Not at all } \\
\text { used }\end{array}$ & $\begin{array}{c}\text { Slightly (a little) } \\
\text { used }\end{array}$ & $\begin{array}{c}\text { Moderately } \\
\text { used }\end{array}$ & $\begin{array}{c}\text { Very much } \\
\text { used }\end{array}$ & $\begin{array}{c}\text { Extremely } \\
\text { used }\end{array}$ \\
\hline$\square_{1}$ & $\square_{2}$ & $\square_{3}$ & $\square_{4}$ & $\square$ \\
\hline
\end{tabular}

39. Please rate the overall quality of the services and facilities at your institution.

\begin{tabular}{|c|c|c|c|}
\hline Poor & Average & Good & Excellent \\
\hline$\square_{1}$ & $\square_{2}$ & $\square_{3}$ & $\square_{4}$ \\
\hline
\end{tabular}

40. If you are not satisfied with the quality of the services or facilities could you please explain any reasons?

\section{Section D - Social experiences}

\section{Living arrangements in New Zealand}

\section{Where are you currently living?}

In a student hostel / hall of residence

In rental accommodation ${ }^{8}$ (e.g., flat).

In your own home.

${ }^{8}$ Accommodation - the place where you are living. 


\section{Community life experiences}

47. Thinking about your experience in New Zealand, please indicate how frequently you engage in following activities: (Please tick one box only for each area)

\begin{tabular}{|c|c|c|c|c|c|c|}
\hline & Never & $\begin{array}{c}\text { Occasio } \\
\text { nally }\end{array}$ & $\begin{array}{c}\text { Six } \\
\text { monthly }\end{array}$ & $\begin{array}{c}\text { Monthl } \\
y\end{array}$ & Weekly & Daily \\
\hline $\begin{array}{l}\text { a. Going to the gym e.g. } \\
\text { weights/classes/fitness }\end{array}$ & $\square_{1}$ & $\square_{2}$ & $\square_{3}$ & $\square_{4}$ & $\square_{5}$ & $\square_{6}$ \\
\hline b. Sports e.g. cricket, soccer & $\square_{1}$ & $\square 2$ & $\square_{3}$ & $\square_{4}$ & $\square_{5}$ & $\square_{6}$ \\
\hline c. Swimming & $\square_{1}$ & $\square_{2}$ & $\square_{3}$ & $\square_{4}$ & $\square_{5}$ & $\square_{6}$ \\
\hline $\begin{array}{l}\text { d. Computer games e.g. PlayStation, } \\
\text { Wi-Fi etc. }\end{array}$ & $\square_{1}$ & $\square_{2}$ & $\square_{3}$ & $\square_{4}$ & $\square_{5}$ & $\square_{6}$ \\
\hline $\begin{array}{l}\text { e. Social networking e.g. Facebook / } \\
\text { Twitter / Orkut }\end{array}$ & $\square_{1}$ & $\square_{2}$ & $\square_{3}$ & $\square_{4}$ & $\square_{5}$ & $\square_{6}$ \\
\hline $\begin{array}{l}\text { f. Talking on Skype with friends or } \\
\text { family }\end{array}$ & 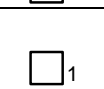 & $\square_{2}$ & $\square_{3}$ & $\square_{4}$ & $\square_{5}$ & $\square_{6}$ \\
\hline g. Watching television & $\square_{1}$ & $\square_{2}$ & $\square 3$ & $\square_{4}$ & $\square_{5}$ & $\square_{6}$ \\
\hline h. Listening to radio & $\square_{1}$ & $\square_{2}$ & $\square_{3}$ & $\square_{4}$ & $\bar{J}_{5}$ & $\square_{6}$ \\
\hline $\begin{array}{l}\text { i. Watching (English language) } \\
\text { movies/DVDs }\end{array}$ & $\square_{1}$ & $\square_{2}$ & $\square_{3}$ & $\square_{4}$ & $\square_{5}$ & $\square_{6}$ \\
\hline j. Watching Indian movies/ DVDs & $\bar{J}_{1}$ & $\square_{2}$ & $\square_{3}$ & $\square_{4}$ & $\square_{5}$ & $\square_{6}$ \\
\hline k. Listening to Indian music & $\bar{J}_{1}$ & $\square_{2}$ & $\square 3$ & $\square 4$ & $J_{5}$ & $\square_{6}$ \\
\hline I. Listening to non-Indian music & $\square_{1}$ & $\square_{2}$ & $\square_{3}$ & $\square_{4}$ & $\square_{5}$ & $\square_{6}$ \\
\hline $\begin{array}{l}\text { m. Reading Indian magazines or non- } \\
\text { academic books }\end{array}$ & $\square_{1}$ & $\square_{2}$ & $\square_{3}$ & $\square_{4}$ & $\square_{5}$ & $\square_{6}$ \\
\hline $\begin{array}{l}\text { n. Reading English language } \\
\text { magazines and non-academic books }\end{array}$ & $\square_{1}$ & $\square_{2}$ & $\square_{3}$ & $\square_{4}$ & $\square_{5}$ & $\square_{6}$ \\
\hline $\begin{array}{l}\text { o. Reading local newspapers (print or } \\
\text { online) }\end{array}$ & $\square_{1}$ & $\square_{2}$ & $\square_{3}$ & $\square_{4}$ & $\square_{5}$ & $\square_{6}$ \\
\hline $\begin{array}{l}\text { p. Reading Indian newspapers (print } \\
\text { or online) }\end{array}$ & $\square_{1}$ & $\square_{2}$ & $\square_{3}$ & $\square_{4}$ & $\square_{5}$ & $\square 6$ \\
\hline q. Indian community activities & $\square_{1}$ & $\square 2$ & $\square_{3}$ & $\square_{4}$ & $\eta_{5}$ & $\square_{6}$ \\
\hline r. Socialising with friends & $\bar{J}_{1}$ & $\square_{2}$ & $\square_{3}$ & $\square 4$ & $\bar{J}_{5}$ & $\square 6$ \\
\hline s. Going to Pubs /nightclubs & $弓_{1}$ & $\square_{2}$ & $\square_{3}$ & $\square_{4}$ & $J_{5}$ & $\square_{6}$ \\
\hline t. Shopping (e.g. going to a mall) & $\square_{1}$ & $\square_{2}$ & $\square_{3}$ & $\square_{4}$ & $\square_{5}$ & $\square_{6}$ \\
\hline $\begin{array}{l}\text { u. Sightseeing/travelling within New } \\
\text { Zealand }\end{array}$ & $\square_{1}$ & $\square_{2}$ & $\square_{3}$ & $\square_{4}$ & $\square_{5}$ & $\square_{6}$ \\
\hline v. Eating Indian food & $\square_{1}$ & $\square_{2}$ & $\square_{3}$ & $\square_{4}$ & $\square_{5}$ & $\square_{6}$ \\
\hline $\begin{array}{l}\text { w. Other significant leisure activity } \\
\text { (please specify) }\end{array}$ & $\square_{1}$ & $\square_{2}$ & $\square_{3}$ & $\square_{4}$ & $\square_{5}$ & $\square_{6}$ \\
\hline
\end{tabular}

48. In what ways have your leisure time activities and habits changed since your arrival in New Zealand? 
49. Do you get any support from Indian community groups?
Yes.
$\ldots$
No.......... $\square$

\section{Section E - Cultural experiences}

\section{Cultural Inclusiveness}

\section{Which religion do you belong to?}

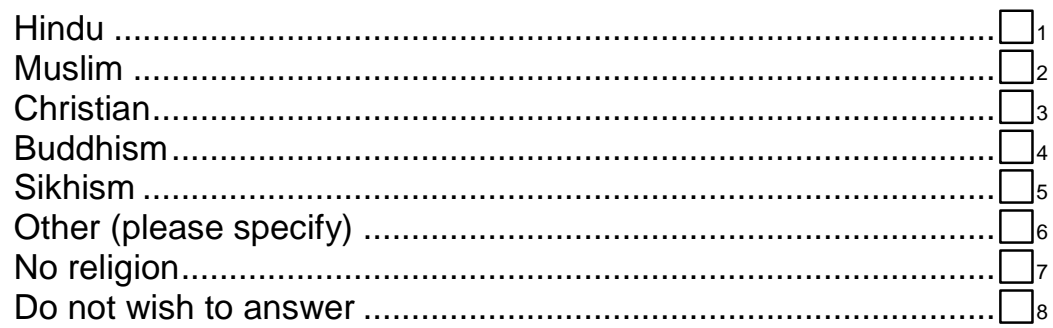

51. Please comment on your experience with maintaining your religion, and carrying out your religious observances in New Zealand".

52. How much do you agree or disagree with the following statements? (Please tick one box only for each statement)

\begin{tabular}{|c|c|c|c|c|c|c|}
\hline & $\begin{array}{l}\text { Strongly } \\
\text { disagree }\end{array}$ & $\begin{array}{c}\text { Mildly } \\
\text { disagree }\end{array}$ & $\begin{array}{c}\text { Neutr } \\
\text { al }\end{array}$ & $\begin{array}{l}\text { Mildly } \\
\text { agree }\end{array}$ & $\begin{array}{c}\text { Strongly } \\
\text { agree }\end{array}$ & $\begin{array}{c}\text { Not } \\
\text { Applicable }\end{array}$ \\
\hline $\begin{array}{l}\text { a. My lecturers/supervisors encourage contact } \\
\text { between international and local students }\end{array}$ & $\square_{1}$ & $\square_{2}$ & $\square_{3}$ & $\square_{4}$ & $\square_{5}$ & $\square_{6}$ \\
\hline $\begin{array}{l}\text { b. The content of my courses is useful for } \\
\text { my future study or employment }\end{array}$ & $\square_{1}$ & $\square_{2}$ & $\square_{3}$ & $\square_{4}$ & $\square_{5}$ & $\square_{6}$ \\
\hline $\begin{array}{l}\text { c. My lecturers/supervisors make special efforts } \\
\text { to help international students }\end{array}$ & $\square_{1}$ & $\square_{2}$ & $\square_{3}$ & $\square_{4}$ & $\square_{5}$ & $\square_{6}$ \\
\hline $\begin{array}{l}\text { d. Cultural differences are respected in my } \\
\text { institution }\end{array}$ & $\square_{1}$ & $\square_{2}$ & $\square_{3}$ & $\square 4$ & $\square_{5}$ & $\square_{6}$ \\
\hline $\begin{array}{l}\text { e. My lecturers/supervisors understand the } \\
\text { problems of international students }\end{array}$ & $\square_{1}$ & $\square_{2}$ & $\square_{3}$ & $\square_{4}$ & $\square_{5}$ & $\square_{6}$ \\
\hline $\begin{array}{l}\text { f. In my classes there is the opportunity for } \\
\text { other students to learn about my culture }\end{array}$ & $\square_{1}$ & $\square_{2}$ & $\square_{3}$ & $\square_{4}$ & $\square_{5}$ & $\square_{6}$ \\
\hline g. I feel included in my class & $\square_{1}$ & $\square_{2}$ & $\square_{3}$ & $\square 4$ & $\square 5$ & $\square_{6}$ \\
\hline $\begin{array}{l}\text { h. My lecturers/supervisors understand } \\
\text { cultural differences in learning styles }\end{array}$ & $\square_{1}$ & $\square_{2}$ & $\square_{3}$ & $\square 4$ & $\square_{5}$ & $\square_{6}$ \\
\hline $\begin{array}{l}\text { i. My classmates are accepting of cultural } \\
\text { differences }\end{array}$ & $\square_{1}$ & $\square_{2}$ & $\square_{3}$ & $\square 4$ & $\square_{5}$ & $\square_{6}$ \\
\hline $\begin{array}{l}\text { j. Students from different cultural groups } \\
\text { work well with each other in my classes }\end{array}$ & $\square_{1}$ & $\square_{2}$ & $\square_{3}$ & $\square 4$ & $\square_{5}$ & $\square_{6}$ \\
\hline
\end{tabular}


53. Has any of the following people treated you unfairly or negatively because of your national, cultural or racial background? Please indicate the answer that best applies to you.

\begin{tabular}{|l|c|c|c|c|c|}
\hline & Never & Seldom & Sometimes & Often & $\begin{array}{c}\text { Very } \\
\text { often }\end{array}$ \\
\hline a. New Zealand students & $\square_{1}$ & $\square_{2}$ & $\square_{3}$ & $\square_{4}$ & $\square_{5}$ \\
\hline b. International students & $\square_{1}$ & $\square_{2}$ & $\square_{3}$ & $\square_{4}$ & $\square_{5}$ \\
\hline c. Academic staff & $\square_{1}$ & $\square_{2}$ & $\square_{3}$ & $\square_{4}$ & $\square_{5}$ \\
\hline $\begin{array}{l}\text { d. Administrative and support staff at your } \\
\text { educational institution }\end{array}$ & $\square_{1}$ & $\square_{2}$ & $\square_{3}$ & $\square_{4}$ & $\square_{5}$ \\
\hline $\begin{array}{l}\text { e. People in the community (e.g., neighbours, } \\
\text { shop assistants, landlords) }\end{array}$ & $\square_{1}$ & $\square_{2}$ & $\square_{3}$ & $\square_{4}$ & $\square_{5}$ \\
\hline
\end{tabular}

54. If you had any specific or unique experience please could you share your experience here.

\section{Friendships}

55. Here are some questions about making friends with New Zealanders. Please indicate your level of agreement or disagreement with each statement. (Please tick one box only for each statement)

\begin{tabular}{|l|c|c|c|c|c|}
\hline & $\begin{array}{c}\text { Strongly } \\
\text { disagree }\end{array}$ & $\begin{array}{c}\text { Mildly } \\
\text { disagree }\end{array}$ & Neutral & $\begin{array}{c}\text { Mildly } \\
\text { agree }\end{array}$ & $\begin{array}{c}\text { Strongly } \\
\text { agree }\end{array}$ \\
\hline a. I would like to have more New Zealand friends & $\square_{1}$ & $\square_{2}$ & $\square_{3}$ & $\square$ & $\square$ \\
\hline $\begin{array}{l}\text { b. New Zealanders should take the first steps to make } \\
\text { friends with international students }\end{array}$ & $\square_{1}$ & $\square_{2}$ & $\square_{3}$ & $\square_{4}$ & $\square$ \\
\hline c. I find it difficult to make friends with New Zealanders & $\square_{1}$ & $\square_{2}$ & $\square_{3}$ & $\square$ & $\square$ \\
\hline $\begin{array}{l}\text { d. New Zealanders do not seem interested in having } \\
\text { international friends }\end{array}$ & $\square_{1}$ & $\square_{2}$ & $\square_{3}$ & $\square_{4}$ & $\square$ \\
\hline $\begin{array}{l}\text { e. My English ability stops me from making New } \\
\text { Zealand friends }\end{array}$ & $\square_{1}$ & $\square_{2}$ & $\square_{3}$ & $\square_{4}$ & $\square$ \\
\hline f. I try my best to make New Zealand friends & $\square_{1}$ & $\square_{2}$ & $\square_{3}$ & $\square$ & $\square$ \\
\hline
\end{tabular}

56. In New Zealand how many close friends do you have from the following groups of people?

(Please tick one box only for each group of people)

\begin{tabular}{|l|c|c|c|c|c|}
\hline & None & One & A few & Some & Many \\
\hline a. New Zealanders (Non-Indian) & $\square_{1}$ & $\square_{2}$ & $\square_{3}$ & $\square_{4}$ & $\square_{5}$ \\
\hline b. New Zealand born Indians & $\square_{1}$ & $\square_{2}$ & $\square_{3}$ & $\square_{4}$ & $\square_{5}$ \\
\hline c. People from India & $\square_{1}$ & $\square_{2}$ & $\square_{3}$ & $\square_{4}$ & $\square_{5}$ \\
\hline d. People from other countries & $\square_{1}$ & $\square_{2}$ & $\square_{3}$ & $\square_{4}$ & $\square$ \\
\hline
\end{tabular}




\section{Life satisfaction in New Zealand}

57. This section is about the sources of support that are available to help you in New Zealand. For each activity, tick the sources of support that are available to you.

(Please tick as many boxes as apply for each type of activity)

\begin{tabular}{|c|c|c|c|c|c|c|c|}
\hline & $\begin{array}{c}\text { Staff at } \\
\text { your } \\
\text { institution }\end{array}$ & $\begin{array}{c}\text { Home } \\
\text { stay } \\
\text { family }\end{array}$ & $\begin{array}{l}\text { NZ (Kiwi) } \\
\text { student } \\
\text { friends }\end{array}$ & $\begin{array}{c}\text { Inter- } \\
\text { national } \\
\text { student } \\
\text { friends } \\
\end{array}$ & $\begin{array}{c}\text { People } \\
\text { from clubs } \\
\text { or the } \\
\text { community }\end{array}$ & $\begin{array}{l}\text { People in } \\
\text { your home } \\
\text { country }\end{array}$ & Others \\
\hline $\begin{array}{l}\text { a. Talk with you if you are upset, } \\
\text { troubled, lonely or homesick }\end{array}$ & $\square_{1}$ & $\square_{2}$ & $\square_{3}$ & $\square_{4}$ & $\square_{5}$ & $\square 6$ & $\square_{7}$ \\
\hline b. Help you if you are sick & $\square_{1}$ & $\square_{2}$ & $\square_{3}$ & $\square_{4}$ & $\square_{5}$ & $\square_{6}$ & $\square_{7}$ \\
\hline $\begin{array}{l}\text { c. Help you with language or } \\
\text { communication problems }\end{array}$ & $\square_{1}$ & $\square_{2}$ & $\square_{3}$ & $\square_{4}$ & $\square_{5}$ & $\square 6$ & $\square_{7}$ \\
\hline $\begin{array}{l}\text { d. Provide information about sexual } \\
\text { health and reproduction }\end{array}$ & $\square_{1}$ & $\square_{2}$ & $\square_{3}$ & $\square_{4}$ & $\square_{5}$ & $\square 6$ & $\square_{7}$ \\
\hline $\begin{array}{l}\text { e. Discuss relationship issues or } \\
\text { concerns with you }\end{array}$ & $\square_{1}$ & $\square_{2}$ & $\square_{3}$ & $\square_{4}$ & $\square_{5}$ & $\square 6$ & $\square_{7}$ \\
\hline f. Help you find your way around & $\square_{1}$ & $\square_{2}$ & $\square_{3}$ & $\square 4$ & $\square_{5}$ & $\square_{6}$ & $\square_{7}$ \\
\hline $\begin{array}{l}\text { g. Help with educational issues or } \\
\text { problems }\end{array}$ & $\square_{1}$ & $\square_{2}$ & $\square 3$ & $\square 4$ & $\square_{5}$ & $\square_{6}$ & $\square_{7}$ \\
\hline $\begin{array}{l}\text { h. Help you become involved in } \\
\text { community activities }\end{array}$ & $\square_{1}$ & $\square_{2}$ & $\square_{3}$ & $\square_{4}$ & $\square_{5}$ & $\square 6$ & $\square_{7}$ \\
\hline $\begin{array}{l}\text { i. Answer questions that you have } \\
\text { about life in New Zealand }\end{array}$ & $\square_{1}$ & $\square_{2}$ & $\square 3$ & $\square 4$ & $\square_{5}$ & $\square_{6}$ & $\square_{7}$ \\
\hline j. Help with your living arrangements & $\square_{1}$ & $\square_{2}$ & $\square_{3}$ & $\square_{4}$ & $\square_{5}$ & $\square_{6}$ & $\square_{7}$ \\
\hline $\begin{array}{l}\text { k. Give advice on banking and } \\
\text { budgeting (financial matters) }\end{array}$ & $\square_{1}$ & $\square_{2}$ & $\square_{3}$ & $\square_{4}$ & $\square_{5}$ & $\square_{6}$ & $\square_{7}$ \\
\hline
\end{tabular}

58. How much do you agree or disagree with the following?

\begin{tabular}{|l|c|c|c|c|c|}
\hline & $\begin{array}{c}\text { Strongly } \\
\text { disagree }\end{array}$ & $\begin{array}{c}\text { Mildly } \\
\text { disagree }\end{array}$ & Neutral & $\begin{array}{c}\text { Mildly } \\
\text { agree }\end{array}$ & $\begin{array}{c}\text { Strongly } \\
\text { agree }\end{array}$ \\
\hline $\begin{array}{c}\text { a. In most ways my life in New Zealand is } \\
\text { close to my ideal (exactly as I would like it) }\end{array}$ & $\square_{1}$ & $\square_{2}$ & $\square_{3}$ & $\square_{4}$ & $\square_{5}$ \\
\hline $\begin{array}{c}\text { b. The conditions of my life in New Zealand } \\
\text { are excellent }\end{array}$ & $\square_{1}$ & $\square_{2}$ & $\square_{3}$ & $\square_{4}$ & $\square_{5}$ \\
\hline c. I am satisfied with my life in New Zealand & $\square_{1}$ & $\square_{2}$ & $\square_{3}$ & $\square_{4}$ & $\square_{5}$ \\
\hline $\begin{array}{c}\text { d. So far I have got the important things I } \\
\text { want from my life in New Zealand }\end{array}$ & $\square_{1}$ & $\square_{2}$ & $\square_{3}$ & $\square_{4}$ & $\square_{5}$ \\
\hline $\begin{array}{c}\text { e. If I could live my life over, I would change } \\
\text { almost nothing about my life in New Zealand }\end{array}$ & $\square_{1}$ & $\square_{2}$ & $\square_{3}$ & $\square_{4}$ & $\square_{5}$ \\
\hline
\end{tabular}


59. Would you like to add any comments on the experience of being an Indian international student in New Zealand?

60. Would you recommend friends or family members to study in New Zealand?

Yes

Thank you for your time and help 


\section{Appendix 2 - Interview Questions}

\section{A Study of the Adjustment Experiences of Indian International students in New Zealand Universities}

(Note: The interview questions may be modified based on the responses of the participants of the Survey questionnaire to be used in the phase one of the research.)

\section{Personal information:}

Age

Gender

Which part of India are you from?

What qualification are you currently doing?

Which field of study?

Which university?

How long have you been in New Zealand?

What was the highest previous qualification?

Why did you choose New Zealand for your study?

Why did you choose this university?

\section{Educational experiences:}

Tell me about your initial impressions of the university life here?

How is your academic learning experience in New Zealand?

Did you face any language difficulties while conversing with native speakers?

What did you do then?

How is your classroom participation?

Did you find any academic differences between India and New Zealand?

Could you explain a few of those differences?

How did you manage this situation?

Would you describe how your teachers help you to overcome academic difficulties?

Other than teachers does anyone help you with these academic issues?

Please explain how did they help you to overcome those issues?

Are you satisfied with your academic progress?

As a doctoral student, are you satisfied with the guidance given by your supervisors?

What did you do then?

What type of guidance you are looking for?

Could you explain how the guidance is different from India?

What type of academic resources you are aspiring?

Are you experiencing any problems in using academic resources?

What did you then?

What aspect of your academic experiences in NZ did you like most?

Could you explain why it is? 
Would you like to make any suggestions for betterment of conditions related to academic aspects?

\section{Experiences with Support Services:}

Did you attend orientation programmes in your university?

How did you come to know about the orientation programmes?

Was the information helpful to you to manage your situation?

If not attended, what did you then?

How did you come to know about the support services in your university?

In what ways you have used support services in you university?

Are you satisfied with the provisions and services?

Could you illustrate any experiences to come to that opinion?

Are the staff of the support services co-operative?

What are the areas they helped you?

Could you illustrate with any example?

Did you have any other source of support, e.g., local Indian community, relatives, friends?

Do you have ample opportunities for social interactions with kiwi students?

What did you do then?

\section{Social and cultural life experiences:}

Are you satisfied with your living arrangements in New Zealand?

Could you explain any reasons for your opinion?

Did you find any part-time work here?

Could you explain why are you not working?

Did you face any difficult experiences while working here?

How did you manage these situations?

Do you have adequate facilities for transportation, sports and recreation?

What did you then?

What do you do normally at leisure times?

Did you find any changes in your leisure time activities or habits since your arrival in New Zealand?

Could you please elaborate your views?

Have you had any good/bad experiences in your social life?

What did you do then?

Do you get ample opportunities to enjoy aspects of Indian culture in New Zealand, e.g., food, films and music?

Do you have adequate facilities to observe your religious practices here?

What did you do then?

What major cultural differences you identify between New Zealand and India?

Do you enjoy the lifestyles of the local people in New Zealand? Could you explain, why? 
Did you notice any interesting aspect of lifestyles of people in New Zealand? Could you explain any?

Do you have any friendships with local New Zealanders?

What do you say about your local New Zealand classmates or friends?

Did you have any bad experience(s) in/out of university area?

Are you satisfied with your life in New Zealand?

Would you recommend your friends or family members to study in New Zealand?

Could you please explain any specific reasons for your decision?

Do you wish to make any suggestions for improvement of conditions of international students in New Zealand?

What are your plans after finishing your studies in New Zealand?

Thank you for your time and participation 
Appendix 3 - Rationale for choosing New Zealand as their study destination (Q.16)(97 respondents)

\begin{tabular}{|c|c|c|}
\hline Category & Sub-category & $\begin{array}{c}\text { Frequency count } \\
(\mathbf{N}=178)\end{array}$ \\
\hline \multirow[t]{6}{*}{ Quality of education } & Good courses & 16 \\
\hline & Good project/topic interested & 11 \\
\hline & Quality education & 10 \\
\hline & Good faculty/Supervisors & 8 \\
\hline & University ranking & 4 \\
\hline & Active research in the field of study & 2 \\
\hline \multirow[t]{2}{*}{ Low cost } & Low fee & 17 \\
\hline & Domestic fee for PhD study & 6 \\
\hline Scholarship & Scholarship & 15 \\
\hline \multirow[t]{8}{*}{ New Zealand image } & Safe and peaceful country & 14 \\
\hline & Beautiful place & 9 \\
\hline & Friendly people/multicultural & 6 \\
\hline & Relaxed lifestyles & 2 \\
\hline & Less population/ standard of living & 5 \\
\hline & Climate and environment & 8 \\
\hline & Adventurous /sports image & 5 \\
\hline & English speaking country & 1 \\
\hline \multirow[t]{2}{*}{ Career prospects } & Better opportunities & 11 \\
\hline & Good work culture & 2 \\
\hline \multirow[t]{7}{*}{ Personal and other factors } & Self interest & 4 \\
\hline & Previous visit experience & 2 \\
\hline & Family ties and friends' influence & 9 \\
\hline & Easy admission procedures & 5 \\
\hline & Easy immigration & 2 \\
\hline & Racism in other countries & 2 \\
\hline & Alternative choice & 2 \\
\hline
\end{tabular}


Appendix 4 - Aspects of academic life enjoyed most by Indian students in New Zealand

(Q.29)(95 respondents)

\begin{tabular}{|c|c|c|}
\hline Category & Sub-category & $\begin{array}{l}\text { Frequency count } \\
\qquad(\mathrm{N}=\mathbf{2 5 6})\end{array}$ \\
\hline \multirow[t]{9}{*}{ Learning approach } & Practical approach & 12 \\
\hline & Presentations/ Discussions & 12 \\
\hline & Independent learning/problem solving & 8 \\
\hline & Interaction & 7 \\
\hline & Critical/analytical thinking & 6 \\
\hline & Group projects & 4 \\
\hline & Study tours & 4 \\
\hline & Different learning style & 2 \\
\hline & Academic meetings & 2 \\
\hline \multirow[t]{3}{*}{ Teaching staff } & Good / friendly/interactive teachers & 28 \\
\hline & Encouraging teachers/supervisors & 7 \\
\hline & Open to new ideas & 1 \\
\hline \multirow[t]{3}{*}{ Resources } & Library/lab/infrastructure facilities & 23 \\
\hline & Collaboration with other researchers & 5 \\
\hline & Access to resources/online journals & 7 \\
\hline \multirow[t]{7}{*}{ Friendly environment } & Friendly environment & 7 \\
\hline & Peer support & 5 \\
\hline & Multicultural classrooms & 4 \\
\hline & Casual classroom & 2 \\
\hline & Clubs \& societies activities & 2 \\
\hline & Small numbers in classes & 2 \\
\hline & Peaceful & 2 \\
\hline \multirow[t]{3}{*}{ Course work material } & Relevant material & 6 \\
\hline & Course work & 4 \\
\hline & Optional workload & 3 \\
\hline
\end{tabular}




\begin{tabular}{|c|c|c|}
\hline & Different course options & 3 \\
\hline & Course structure/up to date syllabus & 4 \\
\hline & Academic/Course module & 2 \\
\hline \multirow[t]{4}{*}{ Teaching } & Ways of teaching & 8 \\
\hline & $\begin{array}{ll}\text { Interactive } & \text { lectures/freedom }\end{array}$ & 4 \\
\hline & Hi-tech teaching & 4 \\
\hline & Quality teaching & 2 \\
\hline \multirow[t]{6}{*}{ Assessment procedures } & Assignments & 6 \\
\hline & Different assessment procedures & 4 \\
\hline & Less importance to written exams & 2 \\
\hline & Online assignments/exams & 2 \\
\hline & Group assignments & 1 \\
\hline & Writing exams & 1 \\
\hline \multirow[t]{19}{*}{ Personal and other factors } & Freedom & 7 \\
\hline & Minimal stress & 5 \\
\hline & Climate & 4 \\
\hline & Relaxed and easy going & 3 \\
\hline & Different educational system & 3 \\
\hline & Project & 2 \\
\hline & Systematic approach & 2 \\
\hline & Openness/transparency & 2 \\
\hline & Opportunity to work & 1 \\
\hline & International contacts & 1 \\
\hline & Respect for students time & 1 \\
\hline & Emphasis on learning & 1 \\
\hline & Dealing with pressure & 1 \\
\hline & Responsibility & 1 \\
\hline & Funding & 1 \\
\hline & Flexibility & 1 \\
\hline & Transparency & 1 \\
\hline & Limited class hours & 1 \\
\hline & More vacation & 1 \\
\hline
\end{tabular}




\begin{tabular}{|l|l|c|}
\hline & Time table & 1 \\
\hline & Learning different things & 1 \\
\hline & Gaining confidence & 1 \\
\hline & Non-compulsory attendance & 1 \\
\hline & Work culture & 1 \\
\hline & Peaceful & 1 \\
\hline & Fun & 1 \\
\hline & Beaches & 1 \\
\hline
\end{tabular}


Appendix 5 - Aspects of academic life that appeared to be challenging to Indian students in New Zealand

(Q.30)(95 respondents)

\begin{tabular}{|c|c|c|}
\hline Category & Sub-category & $\begin{array}{l}\text { Frequency count } \\
\qquad(\mathrm{N}=106)\end{array}$ \\
\hline \multirow[t]{7}{*}{ Assessments } & Writing/completing assignments & 11 \\
\hline & Assessments & 3 \\
\hline & Too much research & 2 \\
\hline & Overlapping of time for submitting & 2 \\
\hline & Lack of proper feed back & 2 \\
\hline & Writing exams & 1 \\
\hline & Grading system & 1 \\
\hline \multirow[t]{5}{*}{ Academic issues } & Academic writing/ writing thesis & 12 \\
\hline & Giving presentations & 2 \\
\hline & Interaction with peers & 1 \\
\hline & Lack of scientific experience & 1 \\
\hline & Lack of resources & 1 \\
\hline \multirow[t]{3}{*}{ Language issues } & Lack of speaking skills & 6 \\
\hline & Understanding language/ kiwi accent & 4 \\
\hline & Coping with language levels & 1 \\
\hline \multirow[t]{5}{*}{ Teaching and learning styles } & Critical thinking/ analysis & 5 \\
\hline & Independent learning & 3 \\
\hline & New methods of presentation & 1 \\
\hline & Group projects & 1 \\
\hline & Referencing & 1 \\
\hline \multirow[t]{6}{*}{ Educational system } & Educational system & 2 \\
\hline & Short time trimesters & 2 \\
\hline & Block course format & 1 \\
\hline & Team dynamics & 1 \\
\hline & Pattern of examinations & 1 \\
\hline & Course work & 1 \\
\hline
\end{tabular}




\begin{tabular}{|c|c|c|}
\hline & Less support from technicians & 1 \\
\hline & Lack of access to research resources & 1 \\
\hline & Long hour classes & 1 \\
\hline \multirow[t]{5}{*}{ Academic staff } & Perceived discrimination & 2 \\
\hline & Dealing with supervisors & 1 \\
\hline & Questioning lecturers & 1 \\
\hline & Average lecturers & 1 \\
\hline & Differences between supervisors & 1 \\
\hline \multirow[t]{3}{*}{ Racism } & Racism & 1 \\
\hline & Arrogance & 1 \\
\hline & Personal vendetta & 1 \\
\hline \multirow[t]{5}{*}{ Other factors - Personal } & Culture shock/loneliness/shyness & 11 \\
\hline & $\begin{array}{l}\text { Emphasis on independent at } \\
\text { university }\end{array}$ & 2 \\
\hline & Too relaxed/Slow & 2 \\
\hline & Lack of opportunities & 1 \\
\hline & Varied experience & 1 \\
\hline \multirow[t]{6}{*}{ Other factors - Social } & Finance & 2 \\
\hline & Weather & 2 \\
\hline & Earthquakes & 1 \\
\hline & Dress code & 1 \\
\hline & New place & 1 \\
\hline & Punctuality & 1 \\
\hline
\end{tabular}




\section{Appendix 6 - Satisfaction of doctoral/ thesis students on different aspects}

(Q.35)(56 respondents)

\begin{tabular}{|c|c|c|c|c|c|c|c|}
\hline S.No. & Question & $\begin{array}{l}\text { Not } \\
\text { appli } \\
\text { cable }\end{array}$ & $\begin{array}{l}\text { Not at } \\
\text { all } \\
\text { satisfie } \\
\quad \text { d }\end{array}$ & $\begin{array}{l}\text { Slightl } \\
\mathbf{y} \\
\text { satisfi } \\
\text { ed }\end{array}$ & $\begin{array}{c}\text { Moder } \\
\text { ately } \\
\text { satisfie } \\
\quad \text { d }\end{array}$ & $\begin{array}{l}\text { Very } \\
\text { satisf } \\
\text { ied }\end{array}$ & $\begin{array}{l}\text { Extrem } \\
\text { ely } \\
\text { satisfied }\end{array}$ \\
\hline 1 & $\begin{array}{l}\text { Access to clear information on } \\
\text { what is required of you as a } \\
\text { doctoral/thesis student }\end{array}$ & $13 \%$ & $0 \%$ & $5 \%$ & $36 \%$ & $38 \%$ & $9 \%$ \\
\hline 2 & $\begin{array}{l}\text { Access to clear information on } \\
\text { your rights as a doctoral/thesis } \\
\text { student }\end{array}$ & $13 \%$ & $4 \%$ & $9 \%$ & $34 \%$ & $30 \%$ & $11 \%$ \\
\hline 3 & $\begin{array}{l}\text { Opportunities for scholarly } \\
\text { interactions with other } \\
\text { postgraduate students }\end{array}$ & $7 \%$ & $7 \%$ & $13 \%$ & $23 \%$ & $34 \%$ & $16 \%$ \\
\hline 4 & $\begin{array}{l}\text { Opportunities to interact with } \\
\text { academic staff other than your } \\
\text { supervisor(s) }\end{array}$ & $9 \%$ & $13 \%$ & $5 \%$ & $25 \%$ & $25 \%$ & $22 \%$ \\
\hline 5 & $\begin{array}{l}\text { Opportunities for skills } \\
\text { development e.g. Writing } \\
\text { proposals }\end{array}$ & $9 \%$ & $7 \%$ & $11 \%$ & $18 \%$ & $36 \%$ & $20 \%$ \\
\hline 6 & $\begin{array}{l}\text { Opportunities to participate and } \\
\text { present at research seminars }\end{array}$ & $13 \%$ & $11 \%$ & $4 \%$ & $20 \%$ & $29 \%$ & $25 \%$ \\
\hline 7 & $\begin{array}{l}\text { The academic guidance provided } \\
\text { by your supervisor(s) }\end{array}$ & $9 \%$ & $4 \%$ & $5 \%$ & $13 \%$ & $34 \%$ & $36 \%$ \\
\hline 8 & $\begin{array}{l}\text { Information and support } \\
\text { provided on using Library } \\
\text { resources }\end{array}$ & $7 \%$ & $0 \%$ & $4 \%$ & $13 \%$ & $25 \%$ & $52 \%$ \\
\hline 9 & $\begin{array}{l}\text { Information and support } \\
\text { provided by Information } \\
\text { Technology Services }\end{array}$ & $9 \%$ & $9 \%$ & $7 \%$ & $16 \%$ & $27 \%$ & $32 \%$ \\
\hline 10 & $\begin{array}{l}\text { Information provided on } \\
\text { different scholarships /Funding } \\
\text { opportunities }\end{array}$ & $7 \%$ & $16 \%$ & $24 \%$ & $11 \%$ & $25 \%$ & $16 \%$ \\
\hline
\end{tabular}


Appendix 7 - Factors helping participants to adjust to their academic work

(Q.31)(65 respondents)

\begin{tabular}{|c|c|c|}
\hline Category & Sub-category & $\begin{array}{l}\text { Frequency count } \\
\qquad(\mathrm{N}=80)\end{array}$ \\
\hline \multirow[t]{20}{*}{ Personal attributes } & Determination & 6 \\
\hline & Personal attitude & 6 \\
\hline & Approaching people & 5 \\
\hline & Previous study and work experience & 3 \\
\hline & Self-realisation & 3 \\
\hline & Passion for subject & 3 \\
\hline & Focus on course work & 3 \\
\hline & Confidence & 3 \\
\hline & Trial and error learning & 2 \\
\hline & Tolerance & 2 \\
\hline & Commitment & 2 \\
\hline & Open mind & 1 \\
\hline & Time management techniques & 1 \\
\hline & Language proficiency & 1 \\
\hline & Volunteering with organisations & 1 \\
\hline & Prior knowledge & 1 \\
\hline & Spending more time & 1 \\
\hline & Proper communication & 1 \\
\hline & Honesty & 1 \\
\hline & Talking to non-New Zealanders & 1 \\
\hline \multirow[t]{13}{*}{ Social support } & Lecturers support & 8 \\
\hline & Peer support & 7 \\
\hline & Friends and past students & 3 \\
\hline & Library staff and facilities & 3 \\
\hline & Discussions with others & 2 \\
\hline & Internet & 2 \\
\hline & Academic clubs & 2 \\
\hline & Student learning support services & 1 \\
\hline & Feedback from peers and lecturers & 1 \\
\hline & Tutorials & 1 \\
\hline & Family members & 1 \\
\hline & Religious group & 1 \\
\hline & International students office & 1 \\
\hline
\end{tabular}


Appendix 8 - Levels of difficulty faced by participants in different academic activities

(Q.27)(104 respondents)

\begin{tabular}{|c|c|c|c|c|c|c|c|}
\hline S.No. & Question & $\begin{array}{l}\text { Not } \\
\text { at all } \\
\text { diffic } \\
\text { ult }\end{array}$ & $\begin{array}{l}\text { Slightly } \\
\text { (a little) } \\
\text { difficult }\end{array}$ & $\begin{array}{l}\text { Modera } \\
\text { tely } \\
\text { difficult }\end{array}$ & $\begin{array}{c}\text { Very } \\
\text { difficult }\end{array}$ & $\begin{array}{l}\text { Extrem } \\
\text { ely } \\
\text { difficult }\end{array}$ & $\begin{array}{c}\text { Not } \\
\text { applicab } \\
\text { le }\end{array}$ \\
\hline 1 & $\begin{array}{l}\text { Understanding } \\
\text { teachers/lecturers }\end{array}$ & $52 \%$ & $28 \%$ & $6 \%$ & $1 \%$ & $0 \%$ & $13 \%$ \\
\hline 2 & $\begin{array}{l}\text { Writing } \\
\text { assignments }\end{array}$ & $19 \%$ & $28 \%$ & $25 \%$ & $11 \%$ & $0 \%$ & $18 \%$ \\
\hline 3 & $\begin{array}{l}\text { Taking notes } \\
\text { during class }\end{array}$ & $51 \%$ & $14 \%$ & $12 \%$ & $0 \%$ & $1 \%$ & $22 \%$ \\
\hline 4 & $\begin{array}{l}\text { Completing } \\
\text { assignments on } \\
\text { time }\end{array}$ & $33 \%$ & $30 \%$ & $13 \%$ & $4 \%$ & $3 \%$ & $17 \%$ \\
\hline 5 & $\begin{array}{l}\text { Working on } \\
\text { group projects }\end{array}$ & $34 \%$ & $24 \%$ & $13 \%$ & $6 \%$ & $2 \%$ & $21 \%$ \\
\hline 6 & $\begin{array}{l}\text { Taking tests or } \\
\text { exams }\end{array}$ & $42 \%$ & $22 \%$ & $6 \%$ & $4 \%$ & $2 \%$ & $24 \%$ \\
\hline 7 & $\begin{array}{l}\text { Making oral } \\
\text { (spoken) } \\
\text { presentations }\end{array}$ & $45 \%$ & $26 \%$ & $16 \%$ & $5 \%$ & $4 \%$ & $4 \%$ \\
\hline 8 & $\begin{array}{l}\text { Managing your } \\
\text { study workload }\end{array}$ & $28 \%$ & $40 \%$ & $18 \%$ & $5 \%$ & $2 \%$ & $7 \%$ \\
\hline 9 & $\begin{array}{l}\text { Asking questions } \\
\text { in class }\end{array}$ & $42 \%$ & $26 \%$ & $16 \%$ & $0 \%$ & $0 \%$ & $17 \%$ \\
\hline 10 & $\begin{array}{l}\text { Thinking } \\
\text { critically } \\
\text { (analytically) }\end{array}$ & $40 \%$ & $33 \%$ & $17 \%$ & $5 \%$ & $2 \%$ & $3 \%$ \\
\hline 11 & $\begin{array}{l}\text { Expressing } \\
\text { yourself } \\
\text { (communicating) } \\
\text { in English }\end{array}$ & $57 \%$ & $31 \%$ & $10 \%$ & $2 \%$ & $0 \%$ & $1 \%$ \\
\hline 12 & $\begin{array}{l}\text { Expressing } \\
\text { (Giving) your } \\
\text { opinions to your } \\
\text { teacher/lecturer }\end{array}$ & $49 \%$ & $37 \%$ & $8 \%$ & $1 \%$ & $1 \%$ & $5 \%$ \\
\hline 13 & $\begin{array}{l}\text { Studying in a } \\
\text { different } \\
\text { educational } \\
\text { system }\end{array}$ & $31 \%$ & $42 \%$ & $20 \%$ & $3 \%$ & $1 \%$ & $3 \%$ \\
\hline
\end{tabular}




\section{Appendix 9 - Suggestions for universities and international students on academic issues}

(13 out of 15 interviewees)

\begin{tabular}{|l|c|}
\hline \multicolumn{1}{|c|}{ Suggestion } & $\begin{array}{c}\text { Number of } \\
\text { responses } \\
\text { (N=27) }\end{array}$ \\
\hline Nuggestions for universities & 7 \\
\hline $\begin{array}{l}\text { Need for information on the academic expectations of New Zealand } \\
\text { educational system }\end{array}$ & 3 \\
\hline Provision of improvement of academic skills & 6 \\
\hline Need for 'Buddy' or mentoring programmes & 3 \\
\hline $\begin{array}{l}\text { Need for understanding the cultural backgrounds of the international } \\
\text { students }\end{array}$ & 1 \\
\hline Need for regular meetings and continuous support & 1 \\
\hline Need for centralised services for international students & 2 \\
\hline & 1 \\
\hline Students should explore the conditions how the system functions & 1 \\
\hline Students should be open and receptive to changes & 1 \\
\hline Students should put extra efforts to succeed & \\
\hline Should attend lectures regularly & 1 \\
\hline
\end{tabular}


Appendix 10 - Quality of the services or facilities available in the universities (Q.37)(104 respondents)

\begin{tabular}{|c|c|c|c|c|c|c|c|}
\hline S.No. & Question & Poor & Average & Good & Excellent & $\begin{array}{l}\text { Don't } \\
\text { know }\end{array}$ & $\begin{array}{c}\text { Not } \\
\text { available }\end{array}$ \\
\hline 1 & International Student Office & $3 \%$ & $10 \%$ & $41 \%$ & $43 \%$ & $2 \%$ & $1 \%$ \\
\hline 2 & Student orientation services & $1 \%$ & $13 \%$ & $41 \%$ & $34 \%$ & $7 \%$ & $4 \%$ \\
\hline 3 & Health services & $4 \%$ & $12 \%$ & $42 \%$ & $30 \%$ & $13 \%$ & $0 \%$ \\
\hline 4 & Financial advice services & $10 \%$ & $13 \%$ & $29 \%$ & $14 \%$ & $30 \%$ & $4 \%$ \\
\hline 5 & Accommodation services & $9 \%$ & $21 \%$ & $21 \%$ & $28 \%$ & $20 \%$ & $1 \%$ \\
\hline 6 & $\begin{array}{l}\text { Vocational (career) or educational } \\
\text { guidance }\end{array}$ & $8 \%$ & $20 \%$ & $27 \%$ & $16 \%$ & $25 \%$ & $4 \%$ \\
\hline 7 & Counselling services & $3 \%$ & $14 \%$ & $28 \%$ & $21 \%$ & $29 \%$ & $5 \%$ \\
\hline 8 & Computing services & $3 \%$ & $13 \%$ & $23 \%$ & $50 \%$ & $8 \%$ & $3 \%$ \\
\hline 9 & Library services & $3 \%$ & $4 \%$ & $27 \%$ & $66 \%$ & $0 \%$ & $0 \%$ \\
\hline 10 & Language laboratories & $3 \%$ & $8 \%$ & $15 \%$ & $16 \%$ & $48 \%$ & $10 \%$ \\
\hline 11 & Learning Support services & $2 \%$ & $11 \%$ & $32 \%$ & $30 \%$ & $21 \%$ & $5 \%$ \\
\hline 12 & "Buddy" or mentor programmes & $8 \%$ & $9 \%$ & $21 \%$ & $21 \%$ & $31 \%$ & $11 \%$ \\
\hline 13 & Sports and Recreational facilities & $5 \%$ & $13 \%$ & $29 \%$ & $38 \%$ & $13 \%$ & $1 \%$ \\
\hline 14 & Clubs and societies & $6 \%$ & $13 \%$ & $25 \%$ & $31 \%$ & $17 \%$ & $8 \%$ \\
\hline 15 & Student Association & $4 \%$ & $15 \%$ & $34 \%$ & $33 \%$ & $11 \%$ & $4 \%$ \\
\hline
\end{tabular}




\section{Appendix 11 - Frequency of participants' leisure time activities in order of preference}

(Q.47)(103 respondents)

\begin{tabular}{|c|c|c|c|c|c|c|}
\hline S.No. & Question & Never & $\begin{array}{l}\text { Occasio } \\
\text { nally }\end{array}$ & Monthly & Weekly & Daily \\
\hline 1 & $\begin{array}{l}\text { Social networking e.g. } \\
\text { Facebook / Twitter / Orkut }\end{array}$ & 4 & 14 & 2 & 7 & 74 \\
\hline 2 & Eating Indian food & 1 & 15 & 9 & 27 & 50 \\
\hline 3 & Listening to Indian music & 3 & 22 & 5 & 22 & 51 \\
\hline 4 & $\begin{array}{l}\text { Talking on Skype with } \\
\text { friends or family }\end{array}$ & 9 & 14 & 6 & 29 & 45 \\
\hline 5 & Watching television & 11 & 20 & 7 & 16 & 48 \\
\hline 6 & Socialising with friends & 3 & 21 & 14 & 45 & 19 \\
\hline 7 & $\begin{array}{l}\text { Watching (English } \\
\text { language) movies/DVDs }\end{array}$ & 4 & 21 & 18 & 42 & 18 \\
\hline 8 & $\begin{array}{l}\text { Reading local newspapers } \\
\text { (print or online) }\end{array}$ & 10 & 31 & 5 & 23 & 34 \\
\hline 9 & $\begin{array}{l}\text { Listening to non-Indian } \\
\text { music }\end{array}$ & 11 & 29 & 8 & 16 & 36 \\
\hline 10 & $\begin{array}{l}\text { Reading Indian } \\
\text { newspapers (print or } \\
\text { online) }\end{array}$ & 23 & 18 & 8 & 18 & 35 \\
\hline 11 & $\begin{array}{l}\text { Shopping (e.g. going to a } \\
\text { mall) }\end{array}$ & 6 & 29 & 24 & 41 & 2 \\
\hline 12 & $\begin{array}{l}\text { Watching Indian movies/ } \\
\text { DVDs }\end{array}$ & 8 & 31 & 19 & 35 & 8 \\
\hline 13 & $\begin{array}{l}\text { Reading English language } \\
\text { magazines and non- } \\
\text { academic books }\end{array}$ & 18 & 36 & 9 & 23 & 17 \\
\hline 14 & Listening to radio & 21 & 43 & 4 & 9 & 25 \\
\hline 15 & $\begin{array}{l}\text { Sightseeing/ travelling } \\
\text { within New Zealand }\end{array}$ & 4 & 65 & 24 & 10 & 0 \\
\hline 16 & $\begin{array}{l}\text { Indian community } \\
\text { activities }\end{array}$ & 31 & 34 & 19 & 15 & 4 \\
\hline 17 & Sports e.g. cricket, soccer & 40 & 31 & 8 & 20 & 2 \\
\hline 18 & $\begin{array}{l}\text { Going to the gym e.g. } \\
\text { weights/classes/fitness }\end{array}$ & 43 & 34 & 4 & 13 & 9 \\
\hline 19 & $\begin{array}{l}\text { Computer games e.g. } \\
\text { PlayStation, Wi-Fi etc. }\end{array}$ & 54 & 19 & 4 & 11 & 13 \\
\hline
\end{tabular}




\begin{tabular}{|c|l|c|c|c|c|c|}
\hline 20 & $\begin{array}{l}\text { Reading Indian magazines } \\
\text { or non-academic books }\end{array}$ & 46 & 29 & 11 & 8 & 9 \\
\hline 21 & Going to Pubs /nightclubs & 44 & 29 & 16 & 13 & 1 \\
\hline 22 & Swimming & 66 & 25 & 7 & 3 & 0 \\
\hline
\end{tabular}


Appendix 12 - Participants' perceptions about different sources of support available in New Zealand

(Q.57)(101 respondents)

\begin{tabular}{|c|c|c|c|c|c|c|c|c|}
\hline S.No. & Question & $\begin{array}{l}\text { Staff } \\
\text { at } \\
\text { your } \\
\text { instit } \\
\text { ution }\end{array}$ & $\begin{array}{l}\text { Home } \\
\text { stay } \\
\text { family }\end{array}$ & $\begin{array}{c}\text { NZ } \\
\text { (Kiwi) } \\
\text { student } \\
\text { friends }\end{array}$ & $\begin{array}{c}\text { Internat } \\
\text { ional } \\
\text { student } \\
\text { friends }\end{array}$ & $\begin{array}{c}\text { People } \\
\text { from } \\
\text { clubs of } \\
\text { the } \\
\text { commu } \\
\text { nity }\end{array}$ & $\begin{array}{c}\text { People } \\
\text { in your } \\
\text { home } \\
\text { country }\end{array}$ & Others \\
\hline 1 & $\begin{array}{l}\text { Talk with you } \\
\text { if you are } \\
\text { upset,troubled, } \\
\text { lonely or } \\
\text { homesick }\end{array}$ & $4 \%$ & $10 \%$ & $3 \%$ & $23 \%$ & $1 \%$ & $47 \%$ & $13 \%$ \\
\hline 2 & $\begin{array}{l}\text { Help you if } \\
\text { you are sick }\end{array}$ & $9 \%$ & $21 \%$ & $4 \%$ & $32 \%$ & $4 \%$ & $12 \%$ & $17 \%$ \\
\hline 3 & $\begin{array}{l}\text { Help you with } \\
\text { language or } \\
\text { communicatio } \\
\text { n problems }\end{array}$ & $23 \%$ & $2 \%$ & $9 \%$ & $22 \%$ & $7 \%$ & $10 \%$ & $28 \%$ \\
\hline 4 & $\begin{array}{l}\text { Provide } \\
\text { information } \\
\text { about sexual } \\
\text { health and } \\
\text { reproduction }\end{array}$ & $17 \%$ & $5 \%$ & $5 \%$ & $10 \%$ & $4 \%$ & $16 \%$ & $41 \%$ \\
\hline 5 & $\begin{array}{l}\text { Discuss } \\
\text { relationship } \\
\text { issues or } \\
\text { concerns with } \\
\text { you }\end{array}$ & $12 \%$ & $8 \%$ & $7 \%$ & $18 \%$ & $4 \%$ & $26 \%$ & $24 \%$ \\
\hline 6 & $\begin{array}{l}\text { Help you find } \\
\text { your way } \\
\text { around }\end{array}$ & $19 \%$ & $10 \%$ & $13 \%$ & $25 \%$ & $2 \%$ & $16 \%$ & $14 \%$ \\
\hline 7 & $\begin{array}{l}\text { Help with } \\
\text { educational } \\
\text { issues or } \\
\text { problems }\end{array}$ & $54 \%$ & $2 \%$ & $6 \%$ & $22 \%$ & $2 \%$ & $5 \%$ & $9 \%$ \\
\hline 8 & $\begin{array}{l}\text { Help you } \\
\text { become } \\
\text { involved in } \\
\text { community } \\
\text { activities }\end{array}$ & $14 \%$ & $7 \%$ & $11 \%$ & $22 \%$ & $21 \%$ & $8 \%$ & $18 \%$ \\
\hline
\end{tabular}




\begin{tabular}{|c|l|c|c|c|c|c|c|c|}
\hline 9 & $\begin{array}{l}\text { Answer } \\
\text { questions that } \\
\text { you have about } \\
\text { life in New } \\
\text { Zealand }\end{array}$ & $21 \%$ & $5 \%$ & $26 \%$ & $21 \%$ & $3 \%$ & $8 \%$ & $16 \%$ \\
\hline 10 & $\begin{array}{l}\text { Help with your } \\
\text { living } \\
\text { arrangements }\end{array}$ & $13 \%$ & $12 \%$ & $10 \%$ & $21 \%$ & $5 \%$ & $12 \%$ & $26 \%$ \\
\hline 11 & $\begin{array}{l}\text { Give advice on } \\
\text { banking and } \\
\text { budgeting } \\
\text { (financial } \\
\text { matters) }\end{array}$ & $14 \%$ & $6 \%$ & $8 \%$ & $22 \%$ & $6 \%$ & $16 \%$ & $27 \%$ \\
\hline
\end{tabular}




\section{Appendix 13 - Participant Information Sheet for the Survey Questionnaire}

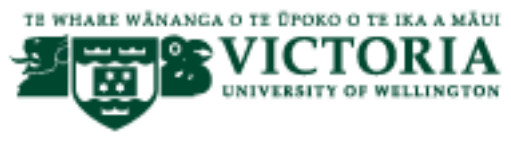

\section{Participant Information Sheet for a Study of 'The Adjustment experiences of Indian International Students in New Zealand Universities'}

Researcher: Johnson Kukatlapalli, School of Languages and Cultures, Victoria University of Wellington.

I am a PhD student in Asian Studies at Victoria University of Wellington. As a part of this degree I am undertaking a research project leading to a thesis. The project I am undertaking is to explore the adjustment experiences of Indian international students at New Zealand universities. The University requires that ethics approval be obtained for research involving human participants.

I am inviting Indian students engaged in University study in New Zealand to participate in this study. Participants will be asked to express their educational, social and cultural experiences in New Zealand through a survey questionnaire. I am thankful to the Ministry of Education, New Zealand for according permission to use the 2007 National Survey questionnaire for my research purpose. I have made certain modifications to suit to the best needs of the current research. It is envisaged that the questionnaire will take approximately 30-40 minutes to complete. The students may have two options regarding filling out and returning the questionnaires. They may return electronic versions or to the researcher's email or hard copies to the researcher by post whichever is convenient to them. Stamped addressed envelops will be supplied to the students who opt for returning the questionnaire by post.

Responses collected will form the basis of my research thesis in which anonymity of the respondents will be strictly maintained. All material collected will be kept confidential. No other person besides me and my supervisors will see the information. The thesis will be submitted for marking to the School of Languages and Cultures and deposited in the university library. It is intended that one or more articles will be submitted for publication in scholarly journals. All the data collected will be destroyed three years after the end of the project.

If you have any questions or would like to receive further information about the project, please contact me at +644 4636487 (Phone), johnson.kukatlapalli@vuw.ac.nz (Email) or my supervisors Prof. Sekhar Bandyopadhyay at +644 4636772 (Phone), sekhar.bandyopadhyay@vuw.ac.nz (Email) and Dr. Stephanie Doyle at +644 4636657 (Phone), stephanie.doyle@ vuw.ac.nz (Email).

Thank you.

Johnson Kukatlapalli

Signed: 


\section{Appendix 14 - Participant Information Sheet for Interviews}

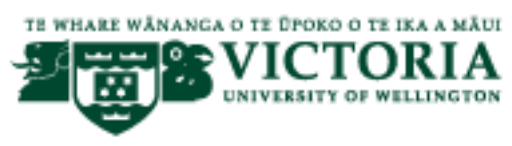

\section{Participant Information Sheet for a Study of 'The Adjustment experiences of Indian International Students in New Zealand Universities'}

Researcher: Johnson Kukatlapalli, School of Languages and Cultures, Victoria University of Wellington.

I am a PhD student in Asian Studies at Victoria University of Wellington. As a part of this degree I am undertaking a research project leading to a thesis. The project I am undertaking is to explore the adjustment experiences of Indian international students at New Zealand universities. The University requires that ethics approval be obtained for research involving human participants.

I am inviting students engaged in University study to participate in this study. Participants will be asked to convey their educational, social and cultural adjustment experiences in New Zealand through interviews. Each interview may take approximately 45-60 minutes. Students' permission will be sought to record their interviews through either audio or through audiovisual mode.

Students involved in this study are free to withdraw at any time from the project before the data are analysed. Students will not be penalized for withdrawing from participation at any stage.

Information received through the responses of the students will form the basis of my research thesis and will be put into a written form on an anonymous basis. All the recorded material will be kept confidential. No other person besides me and my supervisors will examine the information. The thesis will be submitted for marking to the School of Languages and Cultures and deposited in the university library. It is intended that one or more articles will be submitted for publication in scholarly journals. All the recorded data will be destroyed three years after the end of the project.

If you have any questions or would like to receive further information about the project, please contact me at +644 4636487 (Phone), johnson.kukatlapalli@vuw.ac.nz (Email) or my supervisors Prof. Sekhar Bandyopadhyay at +644 4636772 (Phone), sekhar.bandyopadhyay@vuw.ac.nz (Email) and Dr. Stephanie Doyle at +644 4636657 (Phone), Stephanie.doyle@vuw.ac.nz (Email).

Thank you.

Johnson Kukatlapalli

Signed: 


\section{Appendix 15 - Interview Consent Form}

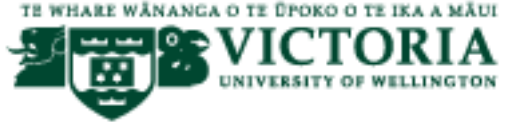

\section{Consent Form for the Interview}

\section{A Study of the Adjustment experiences of Indian International Students in New Zealand Universities}

Researcher: Johnson Kukatlapalli, School of Languages and Cultures, Victoria University of Wellington.

I have read the information relating to the nature and objects of this project and I have understood this information.

I understand that records of any data from me will be kept confidential and that my identity will not be revealed.

I understand that my participation is voluntary and I have the right to withdraw from the project at any time before data collection and analysis are complete.

I understand that if I withdraw from the project, I will not be penalized.

I understand that the information I provide will be used for this research project and any other further use will require my written consent.

I understand that this research project will result in a written thesis and a few academic articles.

I understand that when this research is completed the information obtained will be destroyed.

I agree to take part in this research.

Name:

Email address:

Signed:

Date: 


\begin{tabular}{l|l}
\hline TO & Johnson Kukatlapalli \\
\hline COPY TO & Sekhar Bandyopadhyay \\
\hline FROM & Dr Allison Kirkman, Convener, Human Ethics Committee \\
\hline
\end{tabular}

\begin{tabular}{l|l}
\hline DATE & 15 November 2010 \\
\hline PAGES & 1 \\
\hline
\end{tabular}

\begin{tabular}{l|l}
\hline SUBJECT & $\begin{array}{l}\text { Ethics Approval: No 18089 A study of the adjustment } \\
\text { experiences of Indian International Students in New } \\
\text { Zealand Universities. }\end{array}$ \\
\hline
\end{tabular}

Thank you for your application for ethical approval, which have now been considered by the Standing Committee of the Human Ethics Committee.

Your application has been approved from the above date and this approval continues until 31 March 2012. If your data collection is not completed by this date you should apply to the Human Ethics Committee for an extension to this approval.

Best wishes with the research.

Allison Kirkman

Human Ethics Committee 